\title{
ATLAS DETECTOR AND PHYSICS PERFORMANCE TECHNICAL DESIGN REPORT
}

\section{Chapter 20: Supersymmetry}

\author{
The ATLAS Collaboration ${ }^{*}$
}

\footnotetext{
* See http://atlasinfo.cern.ch/Atlas/Welcome.html for a list of the ATLAS Collaboration membership.

This manuscript has been authored under contract number DE-AC02-98CH10886 with the U.S. Department of Energy. Accordingly, the U.S. Government retains a non-exclusive, royalty-free license to publish or reproduce the published form of this contribution, or allow others to do so, for U.S. Government purposes.
} 


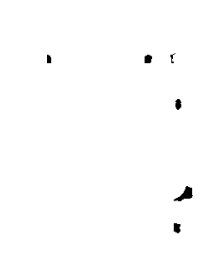




\title{
ATLAS DETECTOR AND PHYSICS PERFORMANCE TECHNICAL DESIGN REPORT
}

\section{Chapter 20: Supersymmetry}

\author{
The ATLAS Collaboration ${ }^{1}$
}

This chapter summarizes the ability of the ATLAS Detector at the CERN LHC to search for SUSY and to make precision measurements of SUSY if it is discovered. The primary emphasis is on detailed studies of selected points in the minimal supergravity model, the minimal gauge mediated SUSY breaking model, and $R$-parity violating models.

This document was written with FrameMaker. A Postscript file of this chapter can be obtained from http://www.cern.ch/Atlas/GROUPS/PHYSICS/TDR/physics_tdr/ printout/Volume_II/Supersymmetry.ps.

\footnotetext{
${ }^{1}$ See http://atlasinfo.cern.ch/Atlas/Welcome.html for a list of the ATLAS Collaboration membership.
} 


\section{Supersymmetry}

\subsection{Introduction}

Supersymmetry - or SUSY - is one of the best motivated extensions of the Standard Model, so the study of SUSY is a primary goal of the LHC. If SUSY exists at the weak scale, $M \sim 1 \mathrm{TeV}$, then discovering evidence for SUSY particles at the LHC seems to be straightforward. Therefore, ATLAS has concentrated on the problems of making precision measurements of SUSY masses (or combinations thereof) and of using these to infer properties of the underlying SUSY model.

While the Standard Model has been tested to an accuracy of order $0.1 \%$ [20-1], the Higgs sector responsible for generating the masses of the $W$ and $Z$ bosons and of the quarks and leptons has not been tested yet. The Higgs boson is the only scalar field in the Standard Model. Scalar fields are special in that loop corrections to their squared masses are quadratically divergent: they are proportional to the cutoff $\Lambda^{2}$, while all other divergences are proportional only to $\log \Lambda^{2}$. Some new mass scale beyond the Standard Model must exist, if only the reduced Planck scale $M_{P}=\left(8 \pi G_{\text {Newton }}\right)^{-1 / 2}=2.4 \times 10^{18} \mathrm{GeV}$ associated with gravity, and the loop corrections to the Higgs mass are naturally of order this scale. This is known as the hierarchy problem [20-2]. The only known solutions - other than accepting an incredible fine tuning - are to embed the Higgs bosons in a supersymmetric theory or to replace the elementary Higgs boson with a dynamical condensate as in technicolor models.

SUSY $[20-3,20-4]$ is the maximal possible extension of the Lorentz group. It has fermionic generators $Q, \bar{Q}$ which satisfy

$$
\begin{gathered}
\{Q, \bar{Q}\}=-2 \gamma_{\mu} P^{\mu} \\
{\left[Q, P^{\mu}\right]=\{Q, Q\}=\{\bar{Q}, \bar{Q}\}=0}
\end{gathered}
$$

where $P^{\mu}$ is the momentum operator and $\gamma_{\mu}$ are the Dirac matrices. SUSY therefore relates particles with the same mass and other quantum numbers differing by $\pm 1 / 2$ unit of spin,

$$
Q \mid \text { boson }\rangle=\mid \text { fermion }\rangle, \quad Q \mid \text { fermion }\rangle=\mid \text { boson }\rangle \text {. }
$$

In the Minimal Supersymmetric extension of the Standard Model (MSSM) each chiral fermion $f_{L, R}$ has a scalar sfermion partner $\tilde{f}_{L, R}$, and each massless gauge boson $A_{\mu}$ with two helicity states \pm 1 has a massless spin-1/2 gaugino partner with helicities $\pm 1 / 2$. There must also be two complex Higgs doublets and their associated Higgsinos to avoid triangle anomalies. The complete list of particles is shown in Tables 20-1 and 20-2. The interactions of SUSY particles are basically obtained from the Standard Model ones by replacing any two lines in a vertex by their SUSY partners; for example, the gluon-quark-quark and gluino-quark-squark couplings are the same. See [20-4] for the construction of the complete Lagrangian.

SUSY provides a solution to the hierarchy problem because it implies an equal number of bosons and fermions, which give opposite signs in loops and so cancel the quadratic divergences. This cancellation works to all orders: since the masses of fermions are only logarithmic divergent, this must also be true for boson masses in a supersymmetric theory. When SUSY is broken, 
Table 20-1 Chiral supermultiplets in the Minimal Supersymmetric Standard Model. The squarks and sleptons come in three generations. The physical Higgs bosons after symmetry breaking are $h, H, A$, and $H^{ \pm}$.

\begin{tabular}{llll}
\hline Name & Spin 0 & Spin 1/2 & $S U(3) \times S U(2) \times U(1)$ \\
\hline squarks, quarks & $\tilde{Q}=\left(\tilde{u}_{L}, \tilde{d}_{L}\right)$ & $Q=\left(u_{L}, d_{L}\right)$ & $(3,2,1 / 6)$ \\
& $\tilde{u}_{R}^{*}$ & $\bar{u}_{R}$ & $(\overline{3}, 1,-2 / 3)$ \\
& $\tilde{d}_{R}^{*}$ & $\bar{d}_{R}$ & $(\overline{3}, 1,1 / 3)$ \\
sleptons, leptons & $\tilde{L}=\left(\tilde{v}, \tilde{e}_{L}\right)$ & $L=\left(\nu, e_{L}\right)$ & $(1,2,-1 / 2)$ \\
& $\tilde{e}_{R}^{*}$ & $\bar{e}_{R}$ & $(1,1,1)$ \\
Higgs, Higgsinos & $H_{u}=\left(H_{u}^{+}, H_{u}^{0}\right)$ & $\tilde{H}_{u}=\left(\tilde{H}_{u}^{+}, \tilde{H}_{u}^{0}\right)$ & $(1,2,1 / 2)$ \\
& $H_{d}=\left(H_{d}^{0}, H_{d}^{-}\right)$ & $\tilde{H}_{d}=\left(\tilde{H}_{d}^{0}, \tilde{H}_{d}^{-}\right)$ & $(1,2,-1 / 2)$ \\
\hline
\end{tabular}

Table 20-2 Vector supermultiplets in the Minimal Supersymmetric Standard Model. After symmetry breaking, the winos and bino mix with the Higgsinos to give four neutralinos $\tilde{\chi}_{i}^{0}$ and two charginos $\tilde{\chi}_{i}^{ \pm}$, and the $W^{0}$ and $B$ mix as in the Standard Model.

\begin{tabular}{llll}
\hline Names & Spin 1/2 & Spin 1 & $S U(3) \times S U(2) \times U(1)$ \\
\hline gluino, gluon & $\tilde{g}$ & $g$ & $(8,1,0)$ \\
winos, $W^{\prime}$ s & $\tilde{W}^{ \pm}, \tilde{W}^{0}$ & $W^{ \pm}, W^{0}$ & $(1,3,0)$ \\
bino, $B$ & $\tilde{B}$ & $B$ & $(1,1,1)$ \\
\hline
\end{tabular}

the corrections to the Higgs and other scalar masses become proportional to the SUSY mass scale rather than the Planck scale. If the Higgs is to be light without unnatural fine tuning, then the SUSY particles should have masses below about $1 \mathrm{TeV}$ [20-5].

The particle content of the Standard Model is elegantly explained by Grand Unified Theories (GUT's), in which the $S U(3), S U(2)$, and $U(1)$ gauge interactions all unify in a simple group such as $S U(5)$. This requires that their respective coupling constants all meet when evolved under the Renormalisation Group Equations (RGE's) to some high mass scale. The precision data at the $Z$ mass collected at LEP and SLC are inconsistent with GUT's using the Standard Model RGE's but are consistent using the SUSY ones provided that the SUSY mass scale is in the $1 \mathrm{TeV}$ range [20-1]. SUSY particles in this mass range are consistent with present direct [20-6, 20-7] and indirect $[20-8]$ limits and with the constraints of fine tuning, and they would be readily observable at the LHC.

SUSY must of course be broken, since superparticles have not been observed: there is certainly no spin-0 selectron degenerate with the electron. Gauge invariance forbids mass terms in the Lagrangian for all Standard Model particles; masses can be introduced only by spontaneous symmetry breaking using the Higgs mechanism. In contrast, mass terms are allowed for all the SUSY particles. Thus, it is possible to parameterise SUSY breaking by introducing by hand SUSY-breaking mass terms for the squarks, sleptons, Higgsinos, and gauginos, all presumably at the $\mathrm{TeV}$ scale. Additional soft terms (bilinear $B$ terms and trilinear $A$ terms with dimension $d<4$ ) consistent with gauge invariance can also be added without reintroducing quadratic divergences. Finally, a Higgsino mass term $\mu$ must be included; this must be of the same order as the SUSY breaking terms even though it is SUSY conserving. 
The requirements of gauge invariance and renormalisability are sufficient to guarantee that the Standard Model Lagrangian conserves baryon and lepton number. In supersymmetric theories it is possible to violate both, potentially leading to disastrous weak-scale proton decay. The unwanted terms can be eliminated by imposing invariance under $R$-parity,

$$
R=(-1)^{3(B-L)+2 S},
$$

where $B, L$, and $S$ are the baryon number, lepton number, and spin. Hence $R=+1$ for all Standard Model particles and $R=-1$ for all SUSY particles. This has the consequence that SUSY particles must be produced in pairs and that the lightest SUSY particle (LSP) is absolutely stable. $R$ parity conservation holds automatically in many GUT models under rather general assumptions [20-9]. Weak-scale proton decay can also be avoided by imposing either baryon or lepton number conservation.

The Minimal Supersymmetric Standard Model (MSSM) is the supersymmetric extension of the Standard Model with the minimal particle content as listed in Tables 20-1 and 20-2 and R-parity conservation. The cancellation of quadratic divergences for scalar masses only requires supersymmetry for the terms with mass dimension four. In the MSSM, SUSY is broken 'by hand' by adding to the Lagrangian all possible soft terms consistent with $S U(3) \times S U(2) \times U(1)$ gauge invariance [20-4]. This includes mass terms for all the superpartners and trilinear $A$ terms:

$$
\begin{aligned}
L_{\text {soft }}= & -m_{H_{d}}^{2}\left|H_{d}\right|^{2}-m_{H_{u}}^{2}\left|H_{u}\right|^{2}+\mu B \varepsilon_{i j}\left(H_{d}^{i} H_{u}^{j}+h . c .\right) \\
& -\frac{1}{2} M_{1} \tilde{\bar{B}} \tilde{B}-\frac{1}{2} M_{2} \tilde{\bar{W}} \tilde{W}-\frac{1}{2} M_{3} \tilde{\bar{g}} \tilde{g} \\
& -M_{\tilde{Q}}^{2}\left(\tilde{u}_{L}^{*} \tilde{u}_{L}+\tilde{d}_{L}^{*} \tilde{d}_{L}\right)-M_{\tilde{U}}^{2} u_{R}^{*} u_{R}-M_{\tilde{D}}^{2} \tilde{d}_{R}^{*} \tilde{d}_{R} \\
& -M_{\tilde{L}^{2}}^{2}\left(\tilde{l}_{L}^{*} \tilde{l}_{L}+\tilde{v}_{L}^{*} \bar{v}_{L}\right)-M_{\tilde{E}}^{2} \tilde{l}_{R}^{*} \tilde{l}_{R} \\
& -\varepsilon_{i j}\left(-\lambda_{u} A_{u} H_{u}^{i} \tilde{Q}^{j} \tilde{u}_{R}^{*}+\lambda_{d} A_{D} H_{d}^{i} \tilde{Q}^{j} \tilde{d}_{R}^{*}+\lambda_{l} A_{E} H_{d}^{i} \tilde{L}^{j} \tilde{l}_{R}^{*}\right)
\end{aligned}
$$

where $Q, L, H_{u}$, and $H_{d}$ denote $S U(2)$ weak doublets as in Table 20-1 and a summation over generations is implied. All the parameters are in general matrices in flavour space and complex; there are a total of 105 new parameters [20-10] in addition to the Standard Model ones. One of these is the SUSY-conserving Higgsino mass $\mu$, which must be of the same order as the SUSY breaking masses. Electroweak symmetry cannot be broken by hand in a similar way, since this would destroy gauge invariance. Instead, it is broken by the Higgs mechanism, giving rise to masses for the quarks, leptons, $W$ and $Z$ bosons. Supersymmetry requires two Higgs doublets and relates the Higgs self-coupling to gauge couplings:

$$
\begin{aligned}
V_{\text {Higgs }}= & \left(m_{H_{d}}^{2}+\mu^{2}\right)\left|H_{d}\right|^{2}+\left(m_{H_{u}}^{2}+\mu^{2}\right)\left|H_{u}\right|^{2}-B \mu\left(\varepsilon_{i j} H_{d}^{i} H_{u}^{j}+h . \mathrm{c} .\right) \\
& +\frac{1}{8}\left(g^{2}+g^{2}\right)\left(\left|H_{d}\right|^{2}-\left|H_{u}\right|^{2}\right)^{2}+\frac{1}{2} g^{2}\left|H_{d}^{i *} H_{u}^{i}\right|^{2}
\end{aligned}
$$

It will be seen in Section 20.2 that the large top Yukawa coupling $\lambda_{t}$ can naturally drive the Higgs squared mass negative, breaking electroweak symmetry.

Once SUSY and electroweak symmetry are broken, particles with the same quantum numbers will in general mix. The gauginos and Higgsinos mix to form two spin- $1 / 2$ 'charginos' $\tilde{\chi}_{i}^{ \pm}$with the mass matrix in the $\left(\tilde{W}^{+}, \tilde{H}^{+}\right)$basis 


$$
M_{\chi^{+}}=\left[\begin{array}{cc}
m_{2} & \sqrt{2} M_{W} \sin \beta \\
\sqrt{2} M_{W} \cos \beta & \mu
\end{array}\right]
$$

and four spin- $1 / 2$ 'neutralinos' $\tilde{\chi}_{i}^{0}$ with the mass matrix in the $\left(B, W^{0}, H_{d}, H_{u}\right)$ basis

$$
M_{\chi^{0}}=\left[\begin{array}{cccc}
m_{1} & 0 & -M_{Z} \cos \beta \sin \theta_{W} & M_{Z} \sin \beta \sin \theta_{W} \\
0 & m_{2} & M_{Z} \cos \beta \cos \theta_{W} & -M_{Z} \sin \beta \cos \theta_{W} \\
-M_{Z} \cos \beta \sin \theta_{W} & M_{Z} \cos \beta \cos \theta_{W} & 0 & -\mu \\
M_{Z} \sin \beta \sin \theta_{W} & -M_{Z} \sin \beta \cos \theta_{W} & -\mu & 0
\end{array}\right] .
$$

In many models

$$
\frac{M_{1}}{\alpha_{1}} \approx \frac{M_{2}}{\alpha_{2}} \approx \frac{M_{3}}{\alpha_{s}}
$$

and $\mu$ is of order $M_{\bar{g}}$, so that the two lighter neutralinos and the lighter chargino are dominantly gaugino, while the heavier states are dominantly Higgsino and weakly coupled to the first two generations.

While the chiral fermions $f_{L}$ and $f_{R}$ must have the same mass by Lorentz invariance, their superpartners $\tilde{f}_{L}$ and $\tilde{f}_{R}$ are scalars with separate masses. Their squared-mass matrix also gets off-diagonal contributions proportional to the fermion mass, e.g.

$$
M_{\tilde{t}}^{2}=\left[\begin{array}{cc}
M_{\tilde{Q}}^{2}+m_{t}^{2}+\frac{1}{6}\left(4 M_{W}^{2}-M_{Z}^{2}\right) \cos 2 \beta & -m_{t}\left(A_{t}-\mu \cot \beta\right) \\
-m_{t}\left(A_{t}-\mu \cot \beta\right) & M_{i_{R}}^{2}+m_{t}^{2}-\frac{2}{3}\left(M_{W}^{2}-M_{Z}^{2}\right) \cos 2 \beta
\end{array}\right] .
$$

The resulting left-right mixing is mainly important for the third generation; the eigenstates are called $\tilde{t}_{1,2}, \vec{b}_{1,2}$, and $\tilde{\tau}_{1,2}$. There can also be mixings among generations of sfermions - including new sources of $C P$ violation. For simplicity all flavour mixing and $L-R$ mixing for the first two generations and all $C P$-violating phases will be ignored here.

Clearly it is not possible to explore the complete parameter space of the MSSM; in the absence of experimental help some theoretical prejudice must be imposed. Presumably supersymmetry should be broken spontaneously rather than by hand and should be unified with gravity. It does not seem possible to construct a phenomenologically acceptable model with spontaneous supersymmetry breaking using only the MSSM fields. Instead, it is necessary to introduce a hidden sector to break SUSY and then to communicate the breaking to the MSSM sector using some messenger interaction that couples to both. In supergravity (SUGRA) models gravity is the sole messenger [20-11]. Then the MSSM masses are scaled by the Planck scale,

$$
M_{\mathrm{MSSM}} \sim \frac{F}{M_{P}},
$$

so the SUSY breaking scale in the hidden sector must be roughly $F-\left(10^{11} \mathrm{GeV}\right)^{2}$. In the minimal SUGRA model, it is assumed that all squarks, sleptons, and Higgs bosons have a common mass $m_{0}$ and that all gauginos have a common mass $m_{1 / 2}$ at the GUT scale. These masses are split when they are run down to the weak scale with the RGE's [20-12]. The squarks and gluinos 
are relatively heavy but nevertheless dominate the SUSY production cross section at LHC energies because of their strong couplings. They typically decay via several steps into the lightest SUSY particle (LSP) $\tilde{\chi}_{1}^{0}$, which is stable. Since the LSP is neutral and weakly interacting, it escapes the detector, giving the characteristic SUSY signature of missing transverse energy $E_{T}^{\text {miss }}$. Signatures for SUGRA models are discussed in Section 20.2.

It is also possible that the SUSY breaking scale in the hidden sector is $F_{m}$ " $\left(10^{11} \mathrm{GeV}\right)^{2}$ and that the MSSM particles get masses through $S U(3) \times S U(2) \times U(1)$ gauge interactions at a messenger scale $M_{m}$ « $M_{P}$. This is known as Gauge Mediated Supersymmetry Breaking (GMSB) [20-13]. Since the gravitino $\tilde{G}$ gets its mass only through gravitational interactions, it is much lighter than all the MSSM particles, which eventually decay into it. In the minimal GMSB model the MSSM masses are proportional to $\Lambda \equiv F_{m} / M_{m}$; the gaugino masses are proportional to the number of messenger fields $N_{5}$, while the scalar masses are proportional to $\sqrt{N_{5}}$. The phenomenology is determined by whether the next lightest SUSY particle (NLSP) is a $\tilde{\chi}_{1}^{0}$ or a $\tilde{\tau}_{1}$ and by whether the NLSP decay $\tilde{\chi}_{1}^{0} \rightarrow \tilde{G} \gamma$ or $\tilde{\tau}_{1} \rightarrow \tilde{G} \tau$ occurs promptly or outside the detector. Signatures for the long-lived $\tilde{\chi}_{1}^{0}$ case are qualitatively similar to those for SUGRA, but the other three cases are quite different. Signatures for the minimal GMSB model are discussed in Section 20.3.

Finally, $R$ parity may be broken, although there are strong constraints on many of the $R$-violating couplings [20-14]. If the $R$-violating couplings are small, then the main effect is to allow the LSP to decay, violating either baryon number, e.g. $\tilde{\chi}_{1}^{0} \rightarrow q \dot{q} q$, or lepton number, e.g. $\tilde{\chi}_{1}^{0} \rightarrow l^{+} l v$, but of course not both baryon and lepton number, since that would give rapid proton decay. Signatures for $R$-parity violation are discussed in Section 20.4 .

Even if the general MSSM is correct, none of these models is likely to be the whole truth, but they do provide self-consistent frameworks in which to test the ability of the ATLAS detector to study supersymmetry at the LHC. In this chapter detailed studies are reported for several particular choices of the parameters in the minimal SUGRA, minimal GMSB, and $R$-parity violating scenarios. Discovery of signals for any of these at the LHC is straightforward. The main problem is not to distinguish SUSY from the Standard Model but to separate the many SUSY processes that occur. Hence, the emphasis here is on partial reconstruction of particular modes, measurement of combinations of masses from kinematic endpoints of the corresponding distributions, and global fits to these to determine the parameters of the underlying model. This approach is quite effective, but it utilises only part of the information that will be available at the LHC if SUSY in fact exists at the weak scale.

Since the main background for SUSY is SUSY itself, it is essential to generate the whole SUSY cross section, not just specific channels of interest. Typically, samples of $10^{5}$ or more events have been generated for each point studied here using either ISAJET [20-15] or SPYTHIA [20-16]. Large samples of Standard Model events are also needed to assess potential backgrounds. Such large event samples necessitate using a fast detector simulation rather than a detailed, GEANTbased one. Most of the results given here are based on ATLFAST [20-17] or comparable particlelevel detector simulations. These correctly describe the gross resolution and acceptance of ATLAS but not the effects of resolution tails, cracks, etc. The backgrounds for SUSY signatures after reasonable cuts appear however to be dominated by real physics events and not by such detector effects. 


\subsection{Supergravity models}

The minimal supergravity (SUGRA) model [20-11] assumes that at the GUT scale all scalars (squarks, sleptons, and Higgs bosons) have a common mass $m_{0}$, all gauginos and Higgsinos have a common mass $m_{1 / 2}$, and all the trilinear Higgs-sfermion-sfermion couplings have a common value $A_{0}$. Such common masses are suggested by the fact that gravity is universal but are sometimes not found in models with realistic GUT-scale dynamics. The remaining parameters at the GUT scale are the bilinear SUSY breaking term $B$ and the SUSY conserving Higgs mass $\mu$. The 26 renormalisation group equations (RGE's) [20-12] are then solved iteratively between the weak and GUT scale. On each iteration the SUSY masses are calculated and used to determine the thresholds at which the masses and other parameters are frozen. Because of the specific form of the RGE's, the squared mass of the Higgs field is driven negative by the large top Yukawa coupling, causing electroweak symmetry breaking but leaving unbroken the col-
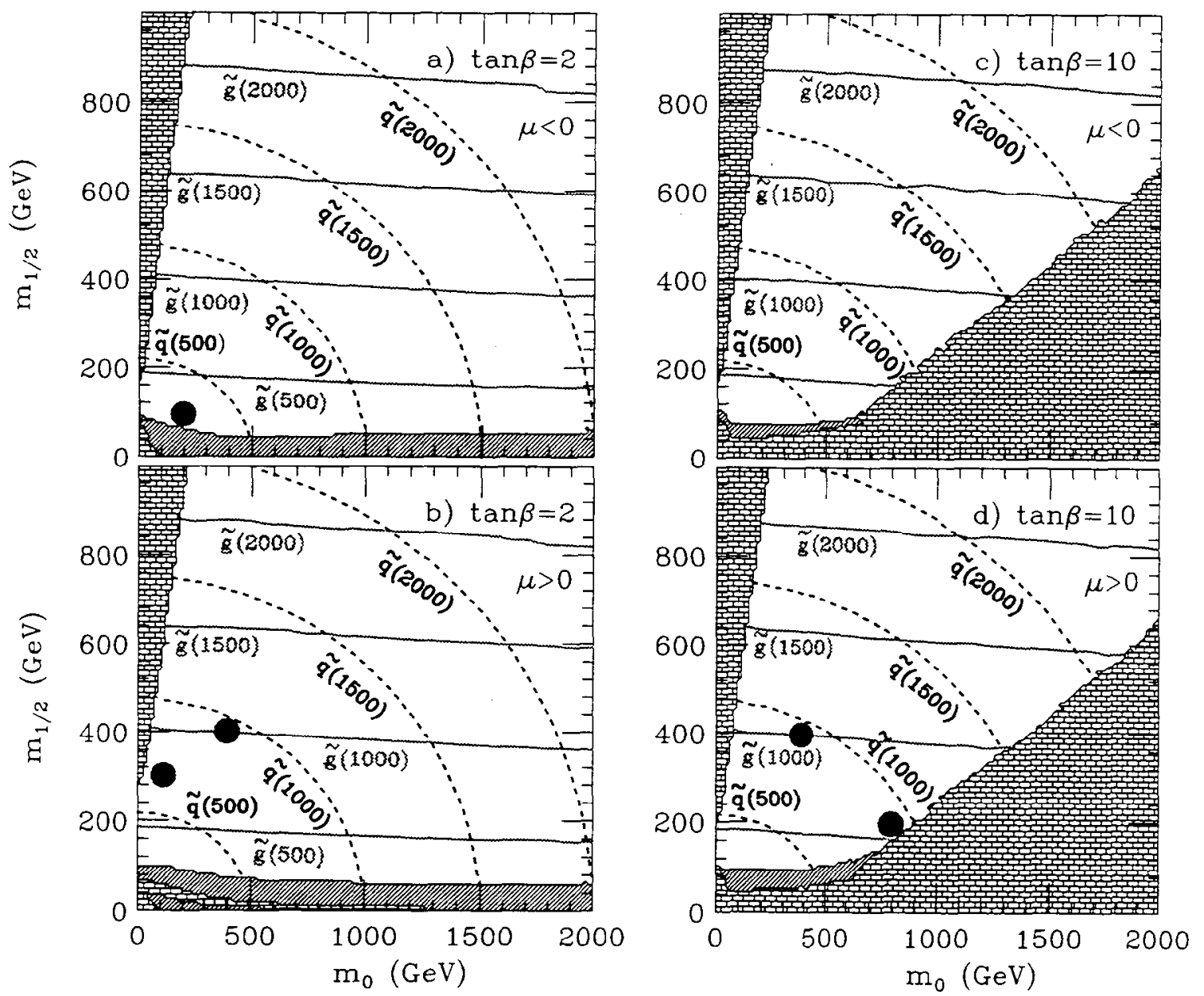

Figure 20-1 Contours showing gluino and squark masses in the $m_{0}, m_{1 / 2}$ plane for $A_{0}=0$ and representative values of $\tan \beta$ in the minimal SUGRA model. The bricked regions at small $m_{0}$ are excluded by the requirement that $\tilde{\chi}_{1}^{0}$ be the LSP. The bricked regions at large $m_{0}$ and $\tan \beta$ are excluded in ISAJET 7.22 by having no electroweak symmetry breaking; this is quite model dependent. The cross-hatched regions were excluded by experiment at the time of Ref. [20-18]. The dots represent the five minimal SUGRA points selected by the LHCC for detailed study by ATLAS and CMS. 

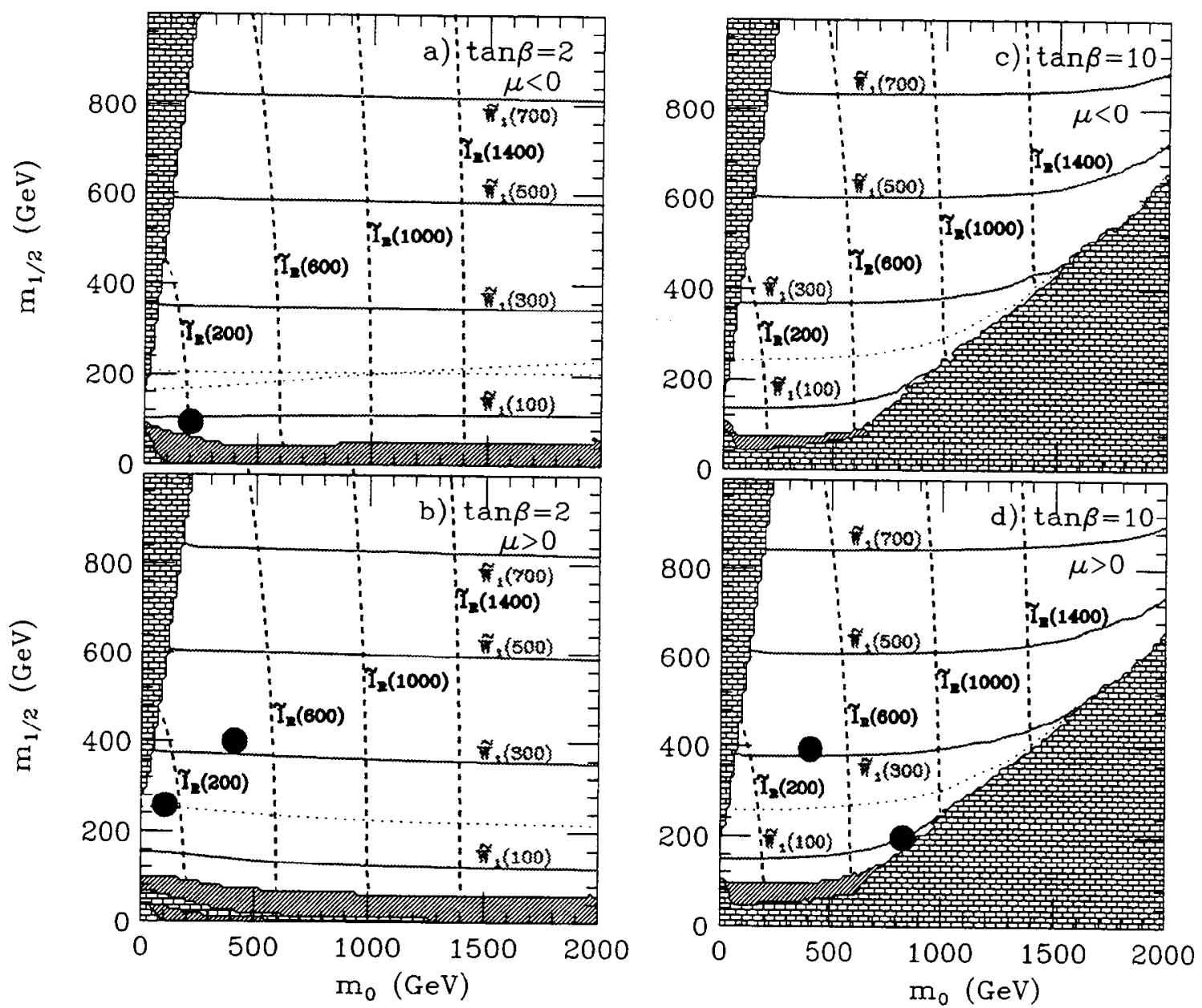

Figure 20-2 Contours showing wino and slepton masses in the $m_{0}, m_{1 / 2}$ plane for $A_{0}=0$ and representative values of $\tan \beta$ in the minimal SUGRA model. See Figure 20-1.

our and electromagnetic interactions. Then $B$ and $\mu^{2}$ can be eliminated in favour of $M_{Z}$ and the ratio $\tan \beta=v_{u} / v_{d}$ of Higgs vacuum expectation values. The parameters of this minimal model are therefore

$$
m_{0}, \quad m_{1 / 2}, \quad A_{0}, \quad \tan \beta, \quad \operatorname{sgn} \mu= \pm 1 .
$$

The masses and mixings of all the SUSY and Higgs particles and hence also all their decays are determined in terms of these parameters. Some representative masses are shown in Figures 20-1 and 20-2. The shaded regions in the figures are excluded by theory or experiment. The dots represent the five minimal SUGRA points selected by the LHC Committee (LHCC) in 1996 for detailed study by the ATLAS and CMS Collaborations.

The parameters of the five LHCC points are listed in Table 20-3; the particle masses are listed in Table 20-4. The total SUSY cross-section ranges between a few picobarn for Points 1 and 2, with a SUSY mass scale of $1 \mathrm{TeV}$, to about one nanobarn for Point 3, with a mass scale of $300 \mathrm{GeV}$. Point 3 is the 'comparison point', selected so that other existing or proposed accelerators could find something. This point is already ruled out: LEP would have discovered the light Higgs with a mass of $68 \mathrm{GeV}$. Points 1 and 5 have light Higgs masses which are excluded by the pre- 
Table 20-3 Parameters of the five minimal SUGRA points selected by the LHCC and a sixth SUGRA point with large $\tan \beta$ added later by ATLAS.

\begin{tabular}{llllll}
\hline Point & $m_{0}(\mathrm{GeV})$ & $m_{1 / 2}(\mathrm{GeV})$ & $A_{0}(\mathrm{GeV})$ & $\tan \beta$ & $\operatorname{sgn} \mu$ \\
\hline 1 & 400 & 400 & 0 & 2 & + \\
2 & 400 & 400 & 0 & 10 & + \\
3 & 200 & 100 & 0 & 2 & - \\
4 & 800 & 200 & 0 & 10 & + \\
5 & 100 & 300 & 300 & 2.1 & + \\
6 & 200 & 200 & 0 & 45 & - \\
\hline
\end{tabular}

liminary limits of the LEP experiments [20-19]. In all three cases the Higgs mass can be increased by increasing $\tan \beta$ to about three. For Point 3 it might also be necessary to increase $m_{0}$ and $m_{1 / 2}$, since the charginos are in a mass region which can be explored by the next LEP run. As an example, the minimal SUGRA point

$$
m_{0}=250 \mathrm{GeV} \quad m_{1 / 2}=125 \mathrm{GeV} \quad A_{0}=0 \quad \tan \beta=5 \quad \mu<0
$$

has a superparticle spectrum generally similar to Point 3 but a light Higgs mass of $98.6 \mathrm{GeV}$. The gluino mass is $350 \mathrm{GeV}$, and it has a high probability to decay via $\tilde{g} \rightarrow \tilde{b}_{1} \bar{b} \rightarrow \tilde{\chi}_{2}^{0} b \bar{b}$; the $\tilde{\chi}_{2}^{0} \rightarrow \tilde{\chi}_{1}^{0} l^{+} l^{-}$branching ratio is smaller but still large enough to be observable. Thus Point 3 remains useful even though it is ruled out by the Higgs search.

Points 1 and 2 both have gluino and squark masses near $1 \mathrm{TeV}$, about the upper limit expected from fine tuning arguments. Point 4 was chosen to be near the boundary for electroweak symmetry breaking, implying that $\mu$ is small so that there is quite strong mixing between gauginos and Higgsinos in Equations 20-1 and 20-2. This boundary turns out to be quite sensitive to the details of how electroweak symmetry breaking is implemented, so the properties of this point are quite model dependent. Point 5 was motivated by cosmology. There is strong evidence for non-baryonic cold dark matter in the universe [20-20], and the LSP is a good candidate for this cold dark matter. Point 5 gives a density of cold dark matter consistent with the universe having the critical density. The small value of $m_{0}$ produces light sleptons and so increases the annihilation cross section for $\tilde{\chi}_{1}^{0}$, reducing the cold dark matter to be consistent with the critical density, $\Omega=1$. (One weakness of the GMSB and $R$-parity violating models considered in Section 20.3 and Section 20.4 is that they do not provide cold dark matter. Conversely, a very weak decay of the LSP via either gravitinos or $R$-parity violating interactions would remove the cold dark matter constraints on SUGRA models.) Point 6 was added later as an example with large $\tan \beta$. This point was selected so that the only two-body gaugino decays are $\tilde{\chi}_{2}^{0} \rightarrow \tilde{\tau}_{1} \tau$ and $\tilde{\chi}_{1}^{ \pm} \rightarrow \tilde{\tau}_{1} v$; these decays therefore dominate and give signatures involving $\tau$ 's instead of the previous signatures. Point 6 is discussed separately in Section 20.2.8.

\subsubsection{Inclusive SUGRA measurements}

The first step in searching for SUSY at the LHC is to look for a deviation from the Standard Model. SUSY events are dominated by the production of gluinos and squarks, which decay through one or more steps to the LSP $\tilde{\chi}_{1}^{0}$, which escapes detection. Thus SUSY events are characterised by multiple jets, leptons, and missing transverse energy $E_{T}^{\mathrm{miss}}$. Since the gluino is a 
Table 20-4 Masses in GeV for the five LHCC SUGRA points and the large $\tan \beta$ SUGRA point listed in Table 20-3. The first and second generation squarks and sieptons are degenerate and so are not listed separately. The SUSY masses for the five LHCC points are from ISAJET 7.22 [20-15]; the Higgs masses are from SPYTHIA 2.08 [20-16] and use the approximate two-loop effective potential. The masses for Point 6 are from ISAJET 7.37 [20-15].

\begin{tabular}{|c|c|c|c|c|c|c|}
\hline Particle & Point 1 & Point 2 & Point 3 & Point 4 & Point 5 & Point 6 \\
\hline$\tilde{g}$ & 1004 & 1009 & 298 & 582 & 767 & 540 \\
\hline$\tilde{\chi}_{1}^{ \pm}$ & 325 & 321 & 96 & 147 & 232 & 152 \\
\hline$\tilde{\chi}_{2}^{ \pm}$ & 764 & 537 & 272 & 315 & 518 & 307 \\
\hline$\tilde{\chi}_{1}^{0}$ & 168 & 168 & 45 & 80 & 122 & 81 \\
\hline$\tilde{\chi}_{2}^{0}$ & 326 & 321 & 97 & 148 & 233 & 152 \\
\hline$\tilde{\chi}_{3}^{0}$ & 750 & 519 & 257 & 290 & 497 & 286 \\
\hline$\tilde{\chi}_{4}^{0}$ & 766 & 538 & 273 & 315 & 521 & 304 \\
\hline$\tilde{u}_{L}$ & 957 & 963 & 317 & 918 & 687 & 511 \\
\hline$\tilde{u}_{R}$ & 925 & 933 & 313 & 910 & 664 & 498 \\
\hline$\tilde{d}_{L}$ & 959 & 966 & 323 & 921 & 690 & 517 \\
\hline $\bar{d}_{R}$ & 921 & 939 & 314 & 910 & 662 & 498 \\
\hline$\tilde{t}_{1}$ & 643 & 710 & 264 & 594 & 489 & 365 \\
\hline$\tilde{t}_{2}$ & 924 & 933 & 329 & 805 & 717 & 517 \\
\hline$\tilde{b}_{1}$ & 854 & 871 & 278 & 774 & 633 & 390 \\
\hline$\tilde{b}_{2}$ & 922 & 930 & 314 & 903 & 663 & 480 \\
\hline$\tilde{e}_{L}$ & 490 & 491 & 216 & 814 & 239 & 250 \\
\hline$\tilde{e}_{R}$ & 430 & 431 & 207 & 805 & 157 & 219 \\
\hline $\bar{v}_{e}$ & 486 & 485 & 207 & 810 & 230 & 237 \\
\hline$\tilde{\tau}_{1}$ & 430 & 425 & 206 & 797 & 157 & 132 \\
\hline$\tilde{\tau}_{2}$ & 490 & 491 & 216 & 811 & 239 & 259 \\
\hline$\tilde{v}_{\tau}$ & 486 & 483 & 207 & 806 & 230 & 218 \\
\hline$h$ & 95 & 116 & 69 & 112 & 93 & 112 \\
\hline$H$ & 1046 & 737 & 379 & 858 & 638 & 157 \\
\hline$A$ & 1044 & 737 & 371 & 859 & 634 & 157 \\
\hline$H^{ \pm}$ & 1046 & 741 & 378 & 862 & 638 & 182 \\
\hline
\end{tabular}

Majorana fermion, it has equal branching ratios into $l^{+} X$ and $l^{-} X$, giving rise to isolated likesign dileptons for which the Standard Model background is small. To estimate the reach for each signature, events were generated for many SUGRA points and for Standard Model processes using ISAJET plus a simplified detector simulation. Events were selected to have [20-18] 

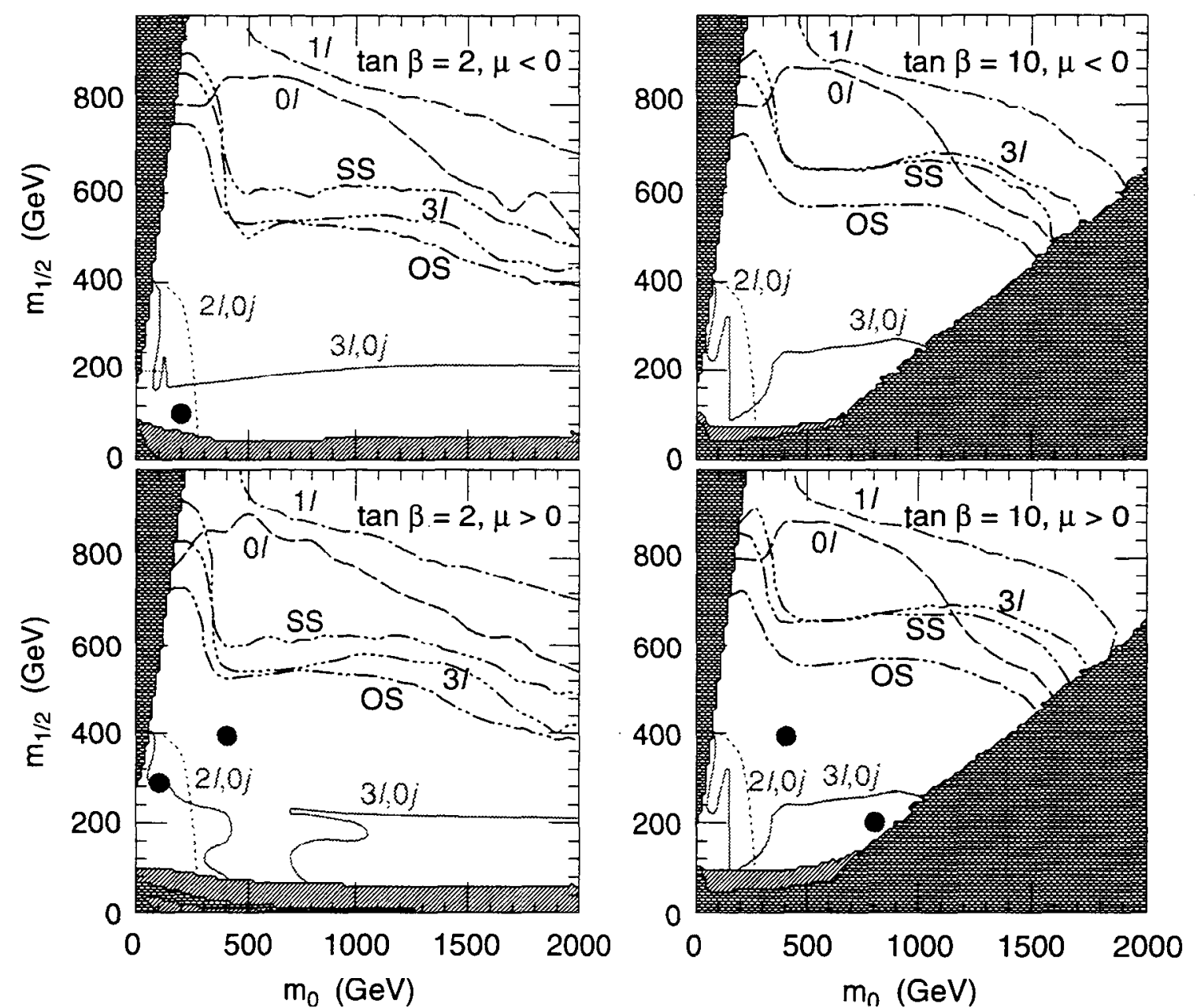

Figure 20-3 Reach for $S / \sqrt{B}>5$ for various SUSY signatures in the SUGRA parameter space with $A_{0}=0$. Ol: Jets, $E_{\mathrm{T}}^{\mathrm{miss}}$, and no leptons. 1l: Jets, $E_{\mathrm{T}}^{\mathrm{miss}}$, and 1 lepton. SS: Same-sign dileptons. OS: Opposite-sign dileptons. 31: trileptons. 31,0j: trileptons with jet veto. 21,0j: dileptons with jet veto. See Figure 20-1 for the explanation of the shaded regions.

- At least two jets with $p_{\mathrm{T}}>E_{\mathrm{T}}^{c}$ and $E_{\mathrm{T}}^{\mathrm{miss}}>E_{\mathrm{T}}^{c}$, where for each SUGRA point the cut parameter $E_{\mathrm{T}}^{c} \geq 100 \mathrm{GeV}$ was adjusted to maximise $S / \sqrt{B}$, where $S$ is the SUSY signal, and $B$ is the background.

- $S_{\mathrm{T}}>0.2$ to reduce the dijet background (where the 'transverse sphericity' $0 \leq S_{\mathrm{T}} \leq 1$ is defined as $2 \lambda_{2} /\left(\lambda_{1}+\lambda_{2}\right), \lambda_{1}$ and $\lambda_{2}$ being the eigenvalues of the $2 \times 2$ sphericity tensor $S_{i j}=\Sigma_{k} p_{k i} p k j$ formed by summing over the transverse momenta of all of the calorimeter cells).

- $p_{\Upsilon}>20 \mathrm{GeV}$ and $\eta<2.5$ for any leptons.

- Transverse mass $M_{\mathrm{T}}\left(l, E_{\mathrm{T}}^{\mathrm{miss}}\right)>100 \mathrm{GeV}$ for the lepton plus jets channel to reduce the $W+$ jets backgrounds.

The resulting reach [20-18], defined by at least 10 signal events and $S / \sqrt{B}>5$ for an integrated luminosity of $10 \mathrm{fb}^{-1}$, is shown Figure 20-3 for the no lepton plus jets plus $E_{\mathrm{T}}^{\text {miss }}$, one lepton plus jets plus $E_{\mathrm{T}}^{\text {miss }}$, same-sign dilepton, opposite-sign dilepton and trilepton channels. By comparing this figure with the previous two, it can be seen that the reach in the jets plus $E_{\mathrm{T}}^{\mathrm{miss}}$ chan- 


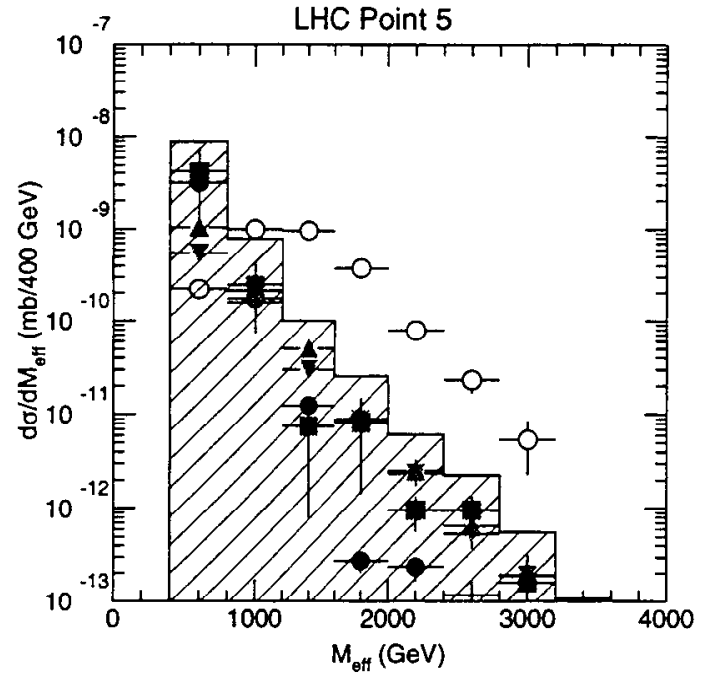

Figure 20-4 $M_{\text {eff }}$ distribution for the Point 5 signal (open circles) and for the sum of all Standard Model backgrounds (histogram); the latter includes $t \bar{t}$ (solid circles), $W+$ jets (triangles), $Z+$ jets (downward triangles), and $Q C D$ jets (squares).

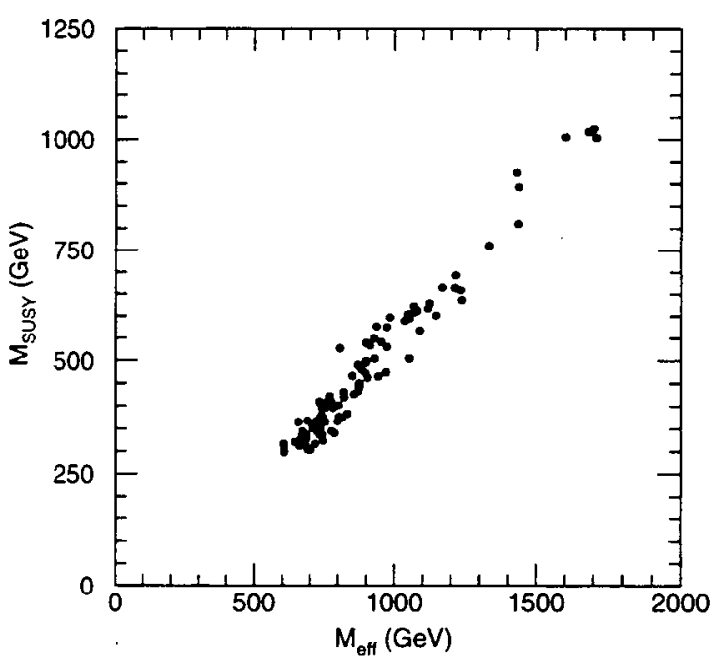

Figure 20-5 Peak of $M_{\text {eff }}$ distribution as a function of. $M_{\mathrm{SUSY}}=\min \left(M_{\bar{g}}, M_{\bar{u}_{R}}\right)$ for various models.

nels extends to squark and gluino masses greater than $2 \mathrm{TeV}$. Similar results were found in the ATLAS Technical Proposal [20-21]. The reach limits involve very hard jets and large $E_{T}^{\mathrm{miss}}$, so they are determined mainly by the squark and gluino production cross sections and not by the detailed detector performance. The reach in the multi-lepton channels extends well beyond $1 \mathrm{TeV}$. Also shown in the figure is the reach for direct gaugino and slepton production in the dilepton and trilepton channels with a central jet veto. These channels generally provide the best reach at the Tevatron [20-6].

After finding one or more such inclusive signals, one would want to obtain a simple estimate of the SUSY masses involved. A good variable [20-22] for this purpose is the 'effective mass', the scalar sum of the missing energy and the transverse momenta of the four hardest jets:

$$
M_{\mathrm{eff}}=E_{\mathrm{T}}^{\mathrm{miss}}+p_{\mathrm{T}, 1}+p_{\mathrm{T}, 2}+p_{\mathrm{T}, 3}+p_{\mathrm{T}, 4} .
$$

This was calculated for the five LHCC SUGRA points listed in Table 20-3 and for the Standard Model backgrounds with the following cuts:

- $E_{\mathrm{T}}^{\text {miss }}>\max \left(100 \mathrm{GeV}, 0.2 M_{\text {eff }}\right)$.

- At least 4 jets with $p_{\mathrm{T}}>50 \mathrm{GeV}$ and $p_{\mathrm{T}, 1}>100 \mathrm{GeV}$.

- Transverse sphericity $S_{\mathrm{T}}>0.2$.

- No muon or isolated electron with $p_{\mathrm{T}}>20 \mathrm{GeV}$ and $\eta<2.5$.

The $M_{\text {eff }}$ distribution after these cuts for the Point 5 signal and the sum of all Standard Model backgrounds is shown in Figure 20-4. Note that $S / B-10$ at large $M_{\text {eff }}$ with an accepted cross section of more than $1 \mathrm{pb}$. For the other points the place where the signal emerges from the background is of course different, but the $S / B$ ratio at large $M_{\text {eff }}$ is similar. Discovering a signal using a variable and simple cuts such as these would be very easy for any of the five LHCC points. This is not very surprising: since squarks and gluinos are strongly interacting, they are 
produced with cross sections comparable to the QCD background at the same $Q^{2}$, so rather simple cuts on $E_{\mathrm{T}}^{\text {miss }}$ and the event topology are sufficient to separate the signal from the Standard Model background.

The peak of the effective mass distribution moves with the mass scale of the SUSY particles. To test this, 100 minimal SUGRA models were selected at random with $m_{0}, m_{1 / 2}$, and $A_{0}$ less than $500 \mathrm{GeV}$ and with the constraint that they have the same light Higgs boson mass as Point 5 within $\pm 3 \mathrm{GeV}$. (In retrospect it was inappropriate to include the Higgs mass constraint, since finding SUSY in this way is far easier than finding a light Higgs boson, but including it has no essential effect.) Figure 20-5 shows a scatter plot of the $M_{\text {eff }}$ peak vs $M_{\text {SUSY }}=\min \left(M_{\tilde{g}}, M_{\bar{u}_{R}}\right)$, which provides a measure of the mass scale of the strongly produced SUSY particles. The spread is remarkably small, about $\pm 10 \%$. The spread might be larger in a more general class of models, but it appears that simple distributions like this will provide a good first estimate of the SUSY mass scale.

One could produce many more inclusive distributions involving various combinations of jets, leptons, and $E_{T}^{\text {miss }}$ and attempt to fit all these to determine the SUGRA model parameters. It is more transparent, however, to use partial reconstruction of exclusive final states to determine precise combinations of masses from kinematic endpoints of distributions as discussed in the following subsections.

\subsubsection{Exclusive SUGRA measurements for moderate $\tan \beta$}

If SUSY exists at the weak scale, it will presumably first be found using inclusive signatures such as the ones discussed in Section 20.2.1. To progress further, and in particular to understand how SUSY is broken, it is important to measure individual masses and decays. In the SUGRA model the decay products of a SUSY particles always include an invisible $\tilde{\chi}_{1}^{0}$, so no mass peaks can be reconstructed directly. It is possible, however, to pick out particular multi-body decay modes and then to determine combinations of masses by measuring the endpoints of the visible mass distributions [20-22, 20-23, 20-24, 20-25, 20-26, 20-27]. For example, in the decay $\bar{\chi}_{2}^{0} \rightarrow \bar{\chi}_{1}^{0} l^{+} l^{-}$the endpoint of the dilepton mass distribution measures $M\left(\tilde{\chi}_{2}^{0}\right)-M\left(\tilde{\chi}_{1}^{0}\right)$. In favourable cases, such measurements can be sufficient to fit the parameters of the model. If a long decay chain can be identified, it is even possible to determine the masses involved without relying on a model. This approach provides a much clearer physical interpretation than a global fit of a model to all possible distributions, although the latter will of course be part of any eventual analysis of data.

The starting point of such analyses is generally to reconstruct a specific mode at the bottom of a decay chain. The SUSY production cross section at the LHC is dominated by gluinos and squarks, which decay mainly through the lighter chargino and two neutralinos. For this reason many of the analyses involve decays of the second neutralino:

- $\tilde{\chi}_{2}^{0} \rightarrow \tilde{\chi}_{1}^{0} l^{+} l^{-}$;

- $\tilde{\chi}_{2}^{0} \rightarrow \tilde{l}^{ \pm} l^{\mp} \rightarrow \tilde{\chi}_{1}^{0} l^{+} l^{-}$;

- $\tilde{\chi}_{2}^{0} \rightarrow \tilde{\chi}_{1}^{0} h \rightarrow \tilde{\chi}_{1}^{0} b \bar{b}$.

Decays of charginos are more difficult to use because they involve either a missing neutrino or light quark jets. If the two-body decays of neutralinos are kinematically allowed, then they generally have substantial branching ratios. If they are not, then the three-body leptonic mode gen- 


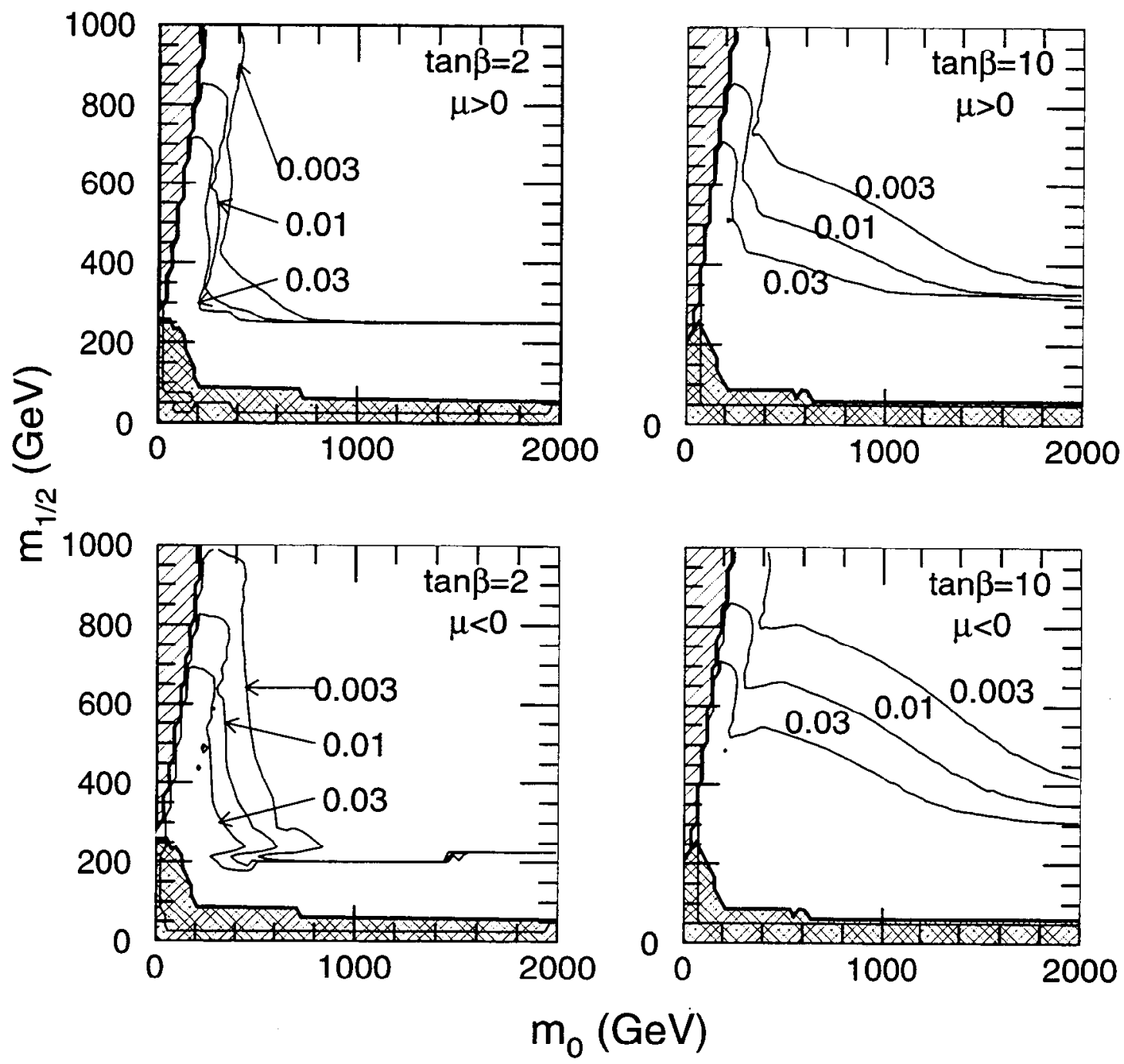

Figure 20-6 Contour plot of branching fraction times cross-section $(B \sigma)$ in pb for $\bar{\chi}_{2}^{0}$ to produce a dilepton pair in the minimal SUGRA model. This figure is based on a more recent implementation of electroweak symmetry breaking than Figures 20-1 - 20-3 that does not give an excluded region at large $m_{0}$ for $\tan \beta=10$.

erally has a branching ratio either comparable to the $Z$ leptonic branching ratio or larger if slepton exchange dominates. All of these modes will be illustrated in the following subsections by analyses for the five SUGRA points listed in Table 20-3.

For moderate $\tan \beta$ at least one of these decay modes is generally available. It is difficult to estimate a precise reach without a detailed study of many points since the backgrounds are dominated by SUSY, but a minimum requirement is that enough events be produced. The SUSY production cross section times combined branching ratio for the sum of all $\tilde{\chi}_{2}^{0}$ dilepton modes $\left(\bar{\chi}_{2}^{0} \rightarrow \tilde{\chi}_{1}^{0} l^{+} l^{-}, \tilde{\chi}_{2}^{0} \rightarrow \tilde{l}^{ \pm} l^{\mp} \rightarrow \tilde{\chi}_{1}^{0} l^{+} l^{-}\right.$, and $\left.\tilde{\chi}_{2}^{0} \rightarrow \tilde{\chi}_{1}^{0} Z \rightarrow \tilde{\chi}_{1}^{0} l^{+} l^{-}\right)$is shown in Figure 20-6, while that for $\tilde{\chi}_{2}^{0} \rightarrow \tilde{\chi}_{1}^{0} h \rightarrow \tilde{\chi}_{1}^{0} b \bar{b}$ is shown in Figure 20-7. $b$-jet tagging assumed a tagging efficiency of 0.6 unless otherwise stated (see Chapter 10).

For very large $\tan \beta$ the splitting among the slepton masses becomes larger, and the only twobody decay available may be $\tilde{\chi}_{2}^{0} \rightarrow \tilde{\tau}_{1}^{ \pm} \tau^{\mp} \rightarrow \tilde{\chi}_{1}^{0} \tau^{+} \tau^{-}$. 


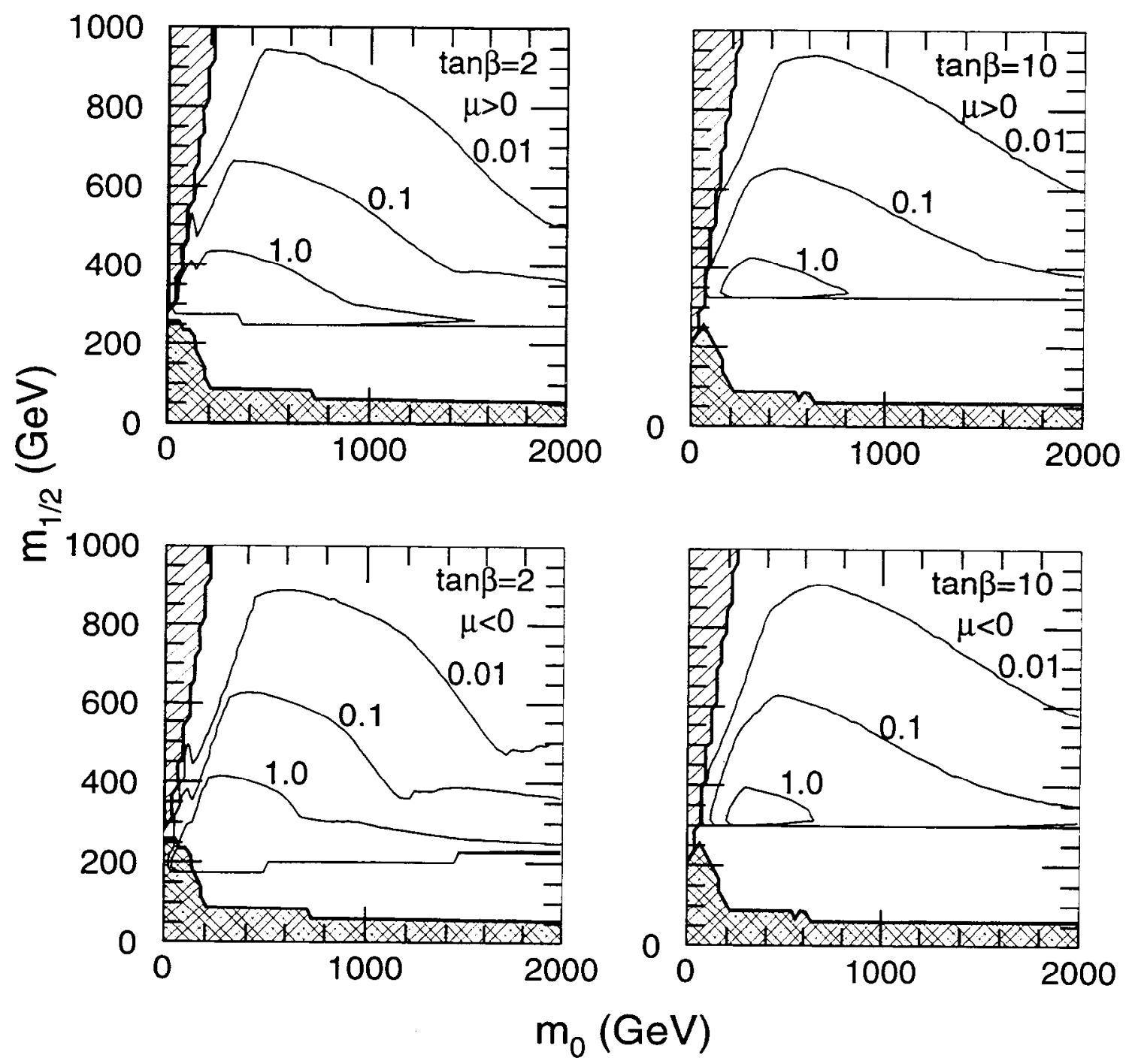

Figure 20-7 Contour plot of $B \sigma$ in pb for $\tilde{\chi}_{2}^{0}$ to decay via $h \rightarrow b \bar{b}$ in the minimal SUGRA model.

If this is the only two-body mode, its branching ratio will be nearly unity. This case is obviously more difficult and will be discussed separately in Section 20.2 .8 below. Given a starting point, one then tries to work back up the decay chain to the primary gluinos and squarks, finding additional exclusive modes and mass constraints. In general the longer the chain that can be identified and reconstructed, the stronger the constraints will be. For this reason, SUGRA models generally are more difficult than GMSB models, which will be discussed in Section 20.3.

Sections 20.2.3-20.2.7 describe such analyses for the five LHCC SUGRA points based on this general approach. Unless otherwise stated, all the analyses described here are based on ISAJET 7.22 [20-15] or SPYTHLA 2.08 [20-16] plus ATLFAST [20-17] or a simple [20-22] particle-level parametrisation of the response of the ATLAS detector. Results are generally shown for integrated luminosities of $10 \mathrm{fb}^{-1}$ to $30 \mathrm{fb}^{-1}$, corresponding to one to three years at low luminosity. Pileup is not properly taken into account, so the results given for high luminosity may be too optimistic. The statistics on the Standard Model backgrounds often correspond to much less than one year. They are sufficient to show that the background is small compared to the signal after the specified cuts but may exhibit large fluctuations. 


\subsubsection{Hr SUGRA signatures}

At Point 3 the light charginos and neutralinos have no two-body decays, so the three-body mode $\tilde{\chi}_{2}^{0} \rightarrow \tilde{\chi}_{1}^{0} l^{+} l^{-}$is relevant. The dominant SUSY cross section is gluino pair production. The $\tilde{b}_{1}$ is $20 \mathrm{GeV}$ lighter than the gluino, but the first and second generation squarks are heavier than it, so the gluinos decay dominantly by $g \rightarrow \tilde{b}_{1} \bar{b}+$ h.c. , the only allowed two-body decay mode. Since the $\tilde{b}_{1}$ is mostly $\tilde{b}_{L}$, it decays mainly into $\tilde{\chi}_{2}^{0}$. Specifically, the branching ratios are [20-22, 20-24]

$$
\begin{aligned}
B\left(\tilde{g} \rightarrow \bar{b}_{1} b+\text { h.c. }\right) & =89 \% \\
B\left(\tilde{b}_{1} \rightarrow \tilde{\chi}_{2} b\right) & =86 \% \\
B\left(\tilde{\chi}_{2} \rightarrow \bar{\chi}_{1}^{0} l^{+} l^{-}\right) & =2 \times 17 \%
\end{aligned}
$$

Thus the SUSY events at Point 3 are characterised by multiple leptons and multiple $b$-jets. While these branching ratios are unusual because the gluino has just one two-body decay mode, it is common for heavy flavour and leptonic decays to be enhanced.

Given these decay modes, events were selected by requiring [20-22, 20-24]:

- A pair of isolated electrons or muons of opposite charge and the same flavour with $p_{\mathrm{T}, l}>10 \mathrm{GeV}$ and $|\eta|<2.5$.

- At least two jets tagged as $b$ 's having $p_{\mathrm{T}}>15 \mathrm{GeV}$ and $|\eta|<2$.

The threshold on lepton $p_{\mathrm{T}}$ is such that only events containing muons will be selected with full efficiency by the trigger [20-28]. For electron pairs the $p_{\mathrm{T}}$ threshold should be raised to $15 \mathrm{GeV}$. Within these kinematic cuts a $b$-tagging efficiency of $60 \%$ and a lepton identification efficiency of $90 \%$ were assumed and included in all distributions; the backgrounds from misidentified leptons and $b$-jets are negligible. These cuts alone are sufficient to reject most of the Standard Model background, so no cut on $E_{\mathrm{T}}^{\text {miss }}$ was used. The resulting dilepton distribution for the signal and for the sum of all Standard Model backgrounds is shown in Figure 20-8. The expected endpoint at

$$
M_{\bar{\chi}_{2}^{0}}-M_{\tilde{\chi}_{1}^{0}}=52.47 \mathrm{GeV}
$$

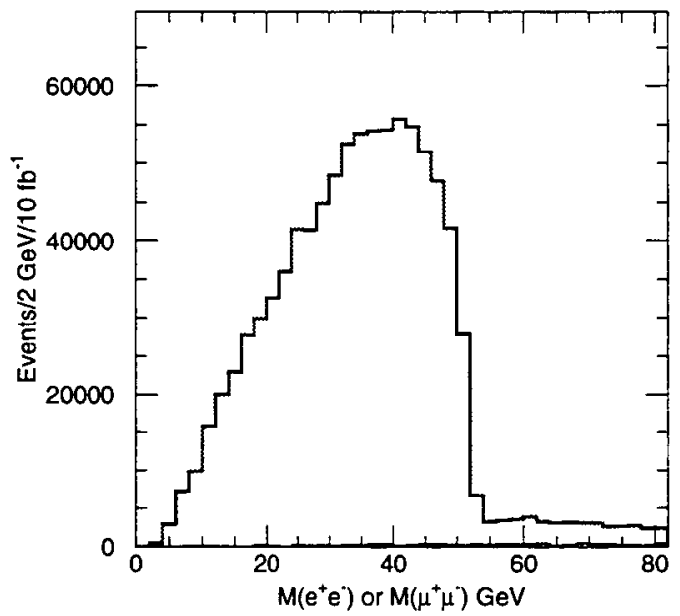

Figure 20-8 Dilepton mass distribution for Point 3 is very clear. The SUSY background comes (solid) and Standard Model background (shaded). mainly from two independent $\tilde{\chi}_{1}^{ \pm}$decays; it is responsible for the tail of events beyond this endpoint. The Standard Model background, which is tiny, comes mainly from $t \dot{t}$ events. If the branching ratios were less favourable, one could plot instead the flavour-subtracted combination $e^{+} e^{-}+\mu^{+} \mu^{-}-e^{ \pm} \mu^{\mp}$ and cancel the background up to statistical fluctuations. Given the high statistics and small background, the endpoint can be determined with an accuracy limited only by systematics, estimated to be $0.1 \%$ or $50 \mathrm{MeV}$. 


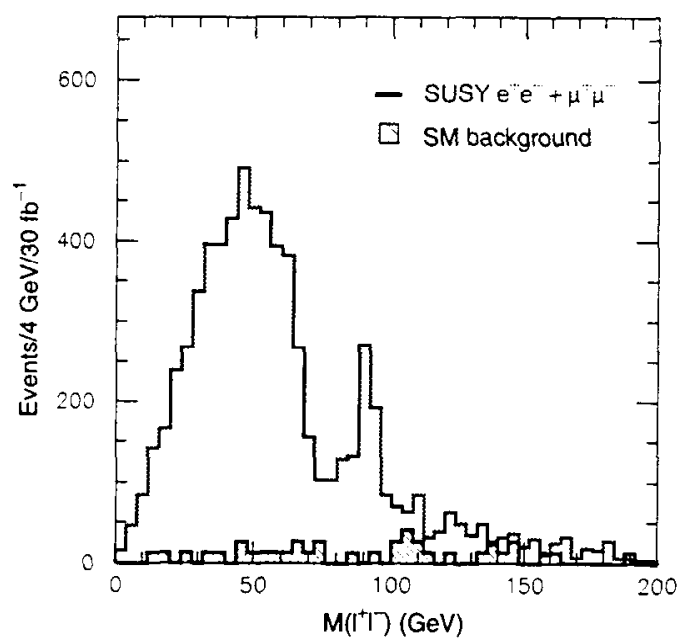

Figure 20-9 Dilepton distribution for Point 4 (solid) and Standard Model background (shaded).

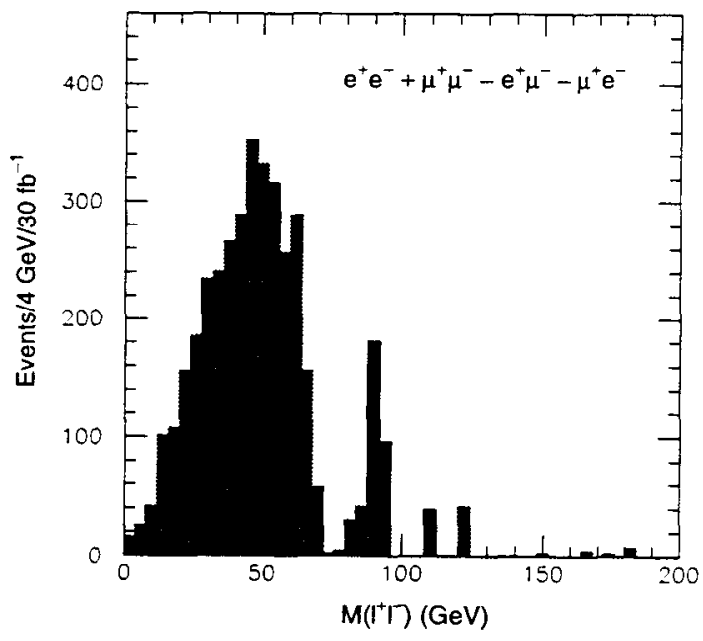

Figure 20-10 Flavour subtracted mass distribution for Point 4.

At Point 4 the light charginos and neutralinos also have no two-body decays. The three-body decay $\tilde{\chi}_{2}^{0} \rightarrow \tilde{\chi}_{1}^{0} l^{+} l^{-}$is again observable although at this point the sleptons are heavy, so the decay occurs through a virtual $Z^{*}$ with a branching ratio of only $2 \times 3 \%$. For the dilepton distribution at Point 4 , events were selected by requiring [20-25]:

- Two isolated, opposite-sign, same-flavour electrons or muons with $p_{\mathrm{T}, 1}>20 \mathrm{GeV}$ and $p_{\mathrm{T}, 2}>10 \mathrm{GeV}$; both leptons are required to have $|\eta|<2.5$.

- $E_{\mathrm{T}}^{\mathrm{miss}}>200 \mathrm{GeV}$.

- At least four jets with $p_{\mathrm{T}}>100,50,50$, and $50 \mathrm{GeV}$ respectively and $|\eta|<3.2$.

After these cuts, there are about 6500 signal events for an integrated luminosity of $30 \mathrm{fb}^{-1}$, with a total Standard Model background of 550 events, mainly from $t i$. The mass distribution is shown in Figure 20-9. Note that in addition to the edge there is also a $Z$ peak in the signal. This comes mainly from heavier gauginos: Point 4 is close to the boundary for radiative electroweak symmetry breaking, so $\mu$ is small. A small $\mu$ implies not only that the heavier charginos and neutralinos are relatively light but also that there is strong mixing between the gauginos and Higgsinos, so they have gauge-like rather than Higgs-like couplings to light flavours. Thus, the total production of the heavier gauginos is larger than is typical for SUGRA models. The heavy gaugino branching ratios into $Z$ 's are also quite large:

$$
\begin{aligned}
B\left(\tilde{\chi}_{2} \rightarrow \tilde{\chi}_{1} Z\right) & =32 \% \\
B\left(\tilde{\chi}_{3}^{0} \rightarrow \tilde{\chi}_{1,2}^{0} Z\right) & =34 \% \\
B\left(\tilde{\chi}_{4}^{0} \rightarrow \tilde{\chi}_{1,2}^{0} Z\right) & =5 \%
\end{aligned}
$$

The endpoint of the dilepton distribution, which again measures $M_{\tilde{\chi}^{0}}-M_{\tilde{\chi}_{1}^{0}}$, can be determined more accurately by plotting the combination $e^{+} e^{-}+\mu^{+} \mu^{-}-e^{ \pm} \mu^{\mp}$, in which two independent decays - e.g., of charginos or of top quarks - cancel. This distribution is shown in Figure 20-10. The 


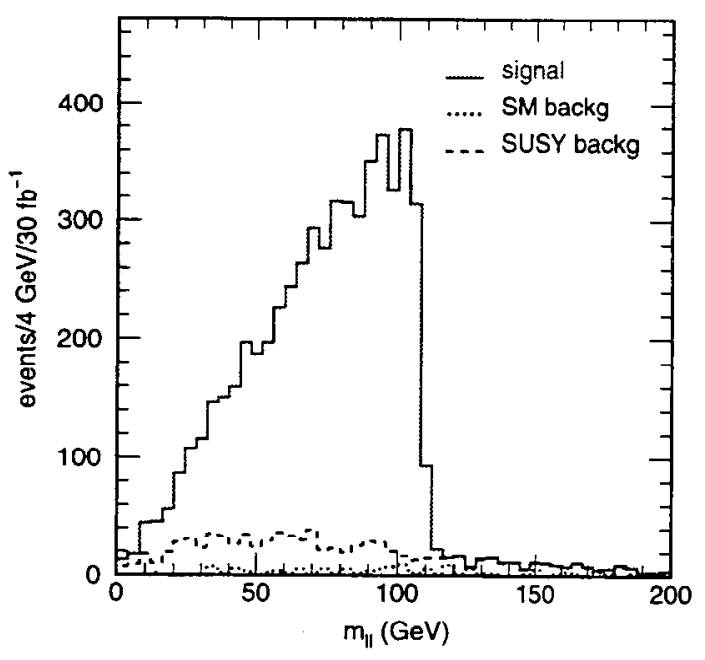

Figure 20-11 Dilepton $\tilde{\chi}_{2}^{0} \rightarrow \tilde{l}_{R}^{t} l^{\mp} \rightarrow \tilde{\chi}_{1}^{0} l^{+} l^{-}$signal at Point 5 (solid), background from other SUSY sources (dashed), and sum of Standard Model backgrounds (dotted) after cuts.

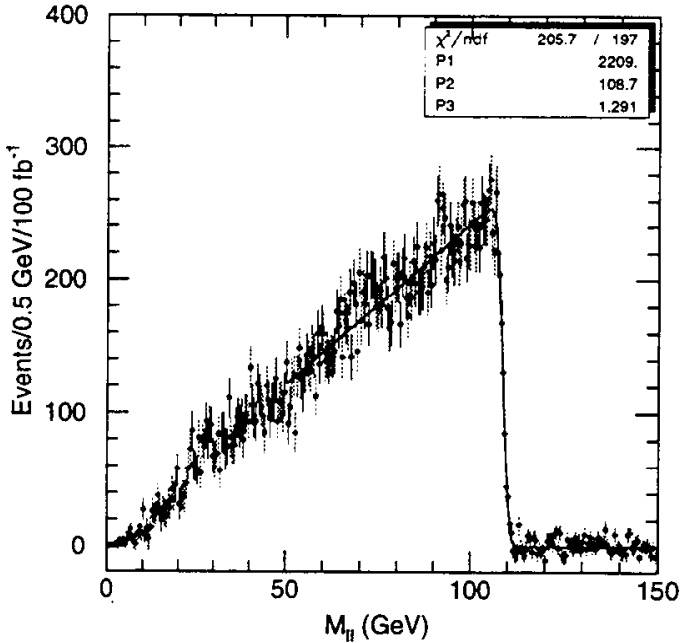

Figure 20-12 Minimum- $\chi^{2}$ fit to flavour-subtracted dilepton signal for Point 5 for an integrated luminosity of $100 \mathrm{fb}^{-1}$.

error on the endpoint for three years at low luminosity was estimated [20-25] using a Kolmogorov test to be $\left(68.13_{-1.0}^{+0.5} \pm 0.07\right) \mathrm{GeV}$, where the first error is statistical and the second is systematic, coming mainly from the $0.1 \%$ uncertainty in the electromagnetic energy scale.

The size of the $Z$ peak relative to the continuum and its $p_{\mathrm{T}}$ distribution can provide information on the masses and mixings of the heavier gauginos, albeit in a model dependent way. A full analysis would require generating many samples of events varying all the parameters of the minimal SUGRA or other SUSY model. To get an approximate indication of the sensitivity, only the $\tilde{\chi}_{2}^{ \pm}$mass was varied holding the other masses fixed. A Kolmogorov test was then used to determine the sensitivity of the $p_{\mathrm{T}}$ distribution of the $Z$ to the $\bar{\chi}_{2}^{ \pm}$mass. This analysis gave $M\left(\tilde{\chi}_{2}^{\frac{ \pm}{2}}\right)=315 \pm 21 \pm 9 \mathrm{GeV}$ for $30 \mathrm{fb}^{-1}[20-25]$.

At Point 5 the right-handed sleptons are relatively light, so the decay $\tilde{\chi}_{2}^{0} \rightarrow \tilde{l}_{R}^{ \pm} l^{\mp} \rightarrow \tilde{\chi}_{1}^{0} l^{+} l^{-}$is open; this is characteristic of SUGRA points that give an amount of cosmological cold dark matter consistent with the universe having the critical density, $\Omega=1$, as predicted by inflation. Since the sleptons are light, the dilepton signal comes from a two-body intermediate state, not from a direct three-body decay. The signal comes mainly from squarks, either directly produced or from gluino decay, so the SUSY events also contain hard jets. Events were therefore selected to have [20-26]

- $E_{\mathrm{T}}^{\mathrm{miss}}>300 \mathrm{GeV}$;

- exactly two opposite-sign, same-flavour electrons or muons with $p_{\mathrm{T}}>10 \mathrm{GeV}$ and $|\eta|<2.5$;

- at least two jets with $p_{\mathrm{T}}>150 \mathrm{GeV}$. 
The $W$ and $Z$ backgrounds are suppressed by the jet requirements and the $t i$ background by the hard kinematics and the leptonic branching ratios. After these cuts, therefore, one is left with 5800 signal events with a background from other SUSY sources of 880 events and a Standard Model background of only 120 events. The mass distribution is shown in Figure 20-11. Because of the two-body kinematics, there is a very sharp edge at

$$
M_{l l}^{\max }=M\left(\tilde{\chi}_{2}^{0}\right) \sqrt{1-\frac{M^{2}\left(\tilde{l}_{R}\right)}{M^{2}\left(\tilde{\chi}_{2}^{0}\right)}} \sqrt{1-\frac{M^{2}\left(\tilde{\chi}_{1}^{0}\right)}{M^{2}\left(\tilde{l}_{R}\right)}}=108.93 \mathrm{GeV}
$$

with a width set by the detector resolution. Most of the small remaining background can be removed by considering the flavour-subtracted combination $e^{+} e^{-}+\mu^{+} \mu^{-}-e^{ \pm} \mu^{\mp}$. The position of this edge can therefore be measured to an estimated precision of $0.5 \mathrm{GeV}$ with an integrated luminosity of $30 \mathrm{fb}^{-1}$ [20-26].

Any difference in the location of this edge for the $e^{+} e^{-}$and $\mu^{+} \mu^{-}$distributions would indicate a difference in the corresponding slepton masses, so it is interesting to measure the edge as accurately as possible [20-29]. Since the slepton is a scalar, the mass distribution before cuts is just given by phase space, i.e.

$$
d M^{2}=\left(M_{l l}^{\max }\right)^{2} \frac{(1+z)}{2} d z
$$

where $z=\cos \theta^{*}$ is the decay angle in the slepton rest frame and is uniformly distributed. Acceptance cuts should not distort this form significantly in the vicinity of the edge. A sample of $1 \times 10^{6}$ events, corresponding to an integrated luminosity of about $75 \mathrm{fb}^{-1}$, was generated with ISAJET 7.37. For this analysis events were selected to have:

- $M_{\text {eff }}>400 \mathrm{GeV}$;

- $E_{\mathrm{T}}^{\text {miss }}>\max \left(100 \mathrm{GeV}, 0.2 M_{\text {eff }}\right)$;

- at least four jets with $p_{\mathrm{T}}>100,50,50,50 \mathrm{GeV}$;

- two isolated, opposite-sign electrons or muons with $p_{\mathrm{T}}>10 \mathrm{GeV}$ and $|\eta|<2.5$.

The dilepton mass for the $e^{+} e^{-}+\mu^{+} \mu^{-}-e^{ \pm} \mu^{\mp}$ combination was then calculated. A minimum- $\chi^{2}$ fit to the signal was made with MINUIT using the mass distribution from Equation 20-6 smeared with a Gaussian resolution; the errors were determined with MINOS [20-30]. It was not possible to generate enough background to include it in the fit with realistic errors. This fit is shown in Figure 22-12; it gave for an integrated luminosity of $100 \mathrm{fb}^{-1}$

$$
M_{l l}^{\max }=108.71_{-0.088}^{+0.087} \mathrm{GeV}
$$

with a resolution of $1.29 \mathrm{GeV}$. A maximum likelihood fit gave a consistent value with slightly smaller errors. The effect of pileup was not taken into account in this analysis. The fitted values are not quite consistent with the expected $108.92 \mathrm{GeV}$, presumably because of small effects from the acceptance cuts and fitting procedure; this has not been investigated. The derivative of the edge position with respect to the slepton mass vanishes at the geometric mean of the $\tilde{\chi}_{1}^{0}$ and $\tilde{\chi}_{2}^{0}$ masses but in general is of order unity. For the masses at Point 5

$$
\frac{d M_{l l}^{\max }}{d M_{\tilde{l}}}=0.478
$$


Given the large sample of $Z \rightarrow l^{+} l^{-}$events, it should be possible to control the systematic errors to less than $0.1 \%$. Therefore, the dilepton endpoint would be sensitive to any difference in the right-handed slepton masses at about the $0.1 \%$ level, assuming of course the same event rates as at this point. The possibility of achieving an understanding of the energy and momentum scales at the level of $0.02 \%$ is investigated in detail in Chapter 9]. If this resolution could be achieved, the uncertainty on the edge measurement would be probably dominated by the statistical error, and by the uncertainties in the modelling of the background. No mass difference is expected in the minimal SUGRA model because of the postulated universality. However, mass differences and lepton flavour mixing can easily be present in more general SUGRA-type models with realistic GUT-scale dynamics and can

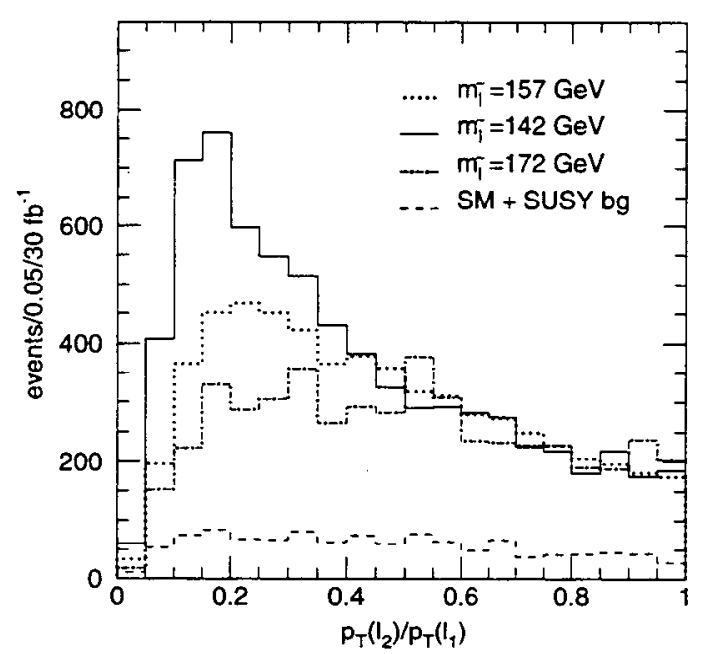

Figure 20-13 Ratio $p_{\mathrm{T}, 2} / p_{\mathrm{T}, 1}$ of the softer to the harder lepton for Point 5 and two other values of the slepton mass. give observable effects both at the LHC and in rare low-energy processes such as $\mu \rightarrow e \gamma$ and $\mu \rightarrow e$ conversion [20-31].,

There is much more information available from the dilepton events. A variable that is particularly sensitive to the slepton mass is $p_{\mathrm{T}, 2} / p_{\mathrm{T}, 1}$, the ratio of the softer to the harder lepton; a slepton mass close to either the $\tilde{\chi}_{1}^{0}$ or the $\tilde{\chi}_{2}^{0}$ mass will obviously make one of the leptons soft. Figure 20-13 shows this ratio for the nominal slepton mass at Point 5, $157 \mathrm{GeV}$, and for two other masses [20-26]. This distribution should be sensitive to changes in the slepton mass of a few $\mathrm{GeV}$ fixing the masses of $\tilde{\chi}_{1}^{0}$ and $\tilde{\chi}_{2}^{0}$. A complete analysis would involve generating samples of events varying all the masses and the $p_{\mathrm{T}}\left(\tilde{\chi}_{2}^{0}\right)$ distribution and then fitting the resulting $M(l l)$, $p_{\mathrm{T}}(l l)$, and $p_{\mathrm{T}, 2} / p_{\mathrm{T}, 1}$ distributions plus the $p_{\mathrm{T}}$ distribution of the $h \rightarrow b \bar{b}$ signal from $\bar{\chi}_{2}^{0} \rightarrow \bar{\chi}_{1}^{0} h$ to be discussed in Section 20.2 .5 below. Such an analysis has not yet been done.

\subsubsection{More complex leptonic SUGRA signatures}

The $\tilde{\chi}_{2}^{0} \rightarrow \tilde{\chi}_{1}^{0} l^{+} l^{-}$or $\tilde{\chi}_{2}^{0} \rightarrow \tilde{l}_{R}^{ \pm} l^{\mp} \rightarrow \tilde{\chi}_{1}^{0} l^{+} l^{-}$decays discussed in the previous section can be the starting point for many more complex analyses that work back up the decay chain to obtain information on other SUSY particles. Examples will be given for all the points having such decays.

\subsubsection{Squark and gluino reconstruction at Point 3}

Most of the dileptons in Figure 20-8 for Point 3 come from the decay chain

$$
\tilde{g} \rightarrow \tilde{b}_{1} \bar{b} \rightarrow \tilde{\chi}_{2}^{0} b \bar{b} \rightarrow \tilde{\chi}_{1}^{0} l^{+} l^{-} b \bar{b}
$$


To reconstruct the gluino and $\tilde{b}_{1}$ in this chain, events with two opposite-sign, same-flavour leptons (lepton $\equiv e, \mu$ in this section) and at least two $b$-jets were selected as before. The dilepton pair was required to be near the endpoint, $45 \mathrm{GeV}<M_{l l}<55 \mathrm{GeV}$. Since the dilepton pair is near its maximum value, both the dilepton pair and the $\tilde{\chi}_{1}^{0}$ are forced to have low momentum in the rest frame of the $\tilde{\chi}_{2}^{0}$, so the $\tilde{\chi}_{2}^{0}$ momentum in the laboratory frame is determined to be

$\vec{p}_{\bar{\chi}_{2}^{0}}=\left(1+\frac{M_{\bar{\chi}_{1}^{0}}}{M_{l l}}\right) \vec{p}_{l l}$

Of course the $\tilde{\chi}_{1}^{0}$ mass is still unknown and must be determined from an overall fit; a first approximation would be to estimate it from the dilepton endpoint assuming $M\left(\tilde{\chi}_{2}^{0}\right) \approx 2 M\left(\tilde{\chi}_{1}^{0}\right)$, as is generally true in models with gauge coupling unification. The energies and momenta of the tagged $b$-jets were corrected to the true energy (see Section 12.5.1.3), to account for losses from neutrinos and from energy leaking out of the $R=0.4$ cone. This correction was actually derived for the $h \rightarrow b \bar{b}$ analysis and will be discussed in Section 20.2 .5 below. The reconstructed $\tilde{\chi}_{2}^{0}$ momentum was combined with one $b$-jet to form the $\tilde{b}_{1}$ mass and then with a second $b$-jet to form the $\tilde{g}$ mass. Since the mass difference is small, the resolution on the difference is better than that on the individual masses; the kinematics is analogous to that familiar from $D^{*} \rightarrow D \pi$. The resulting scatter plot assuming the correct $\tilde{\chi}_{1}^{0}$ mass is shown in Figure 20-14, and the projections on the two axes are shown in Figures 20-15 and 20-16. While

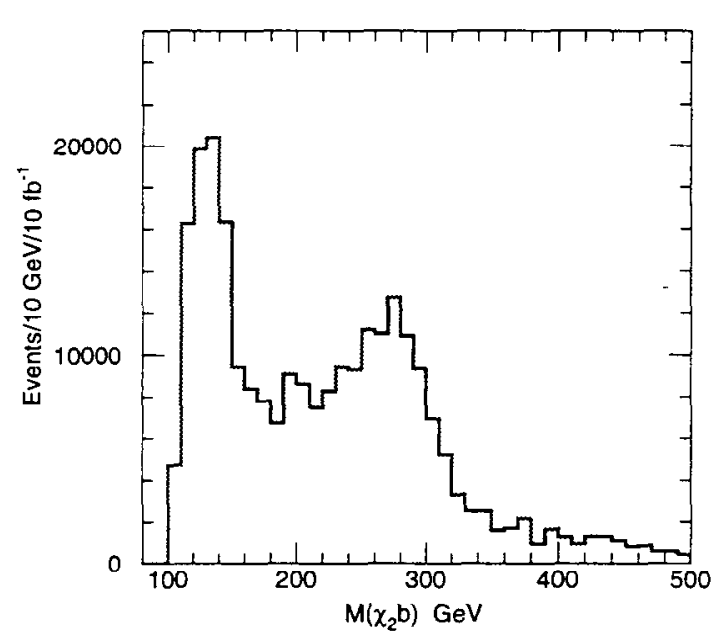

Figure 20-15 $M\left(\tilde{b}_{1}\right)$ projection of the scatter plot in Figure 20-14.

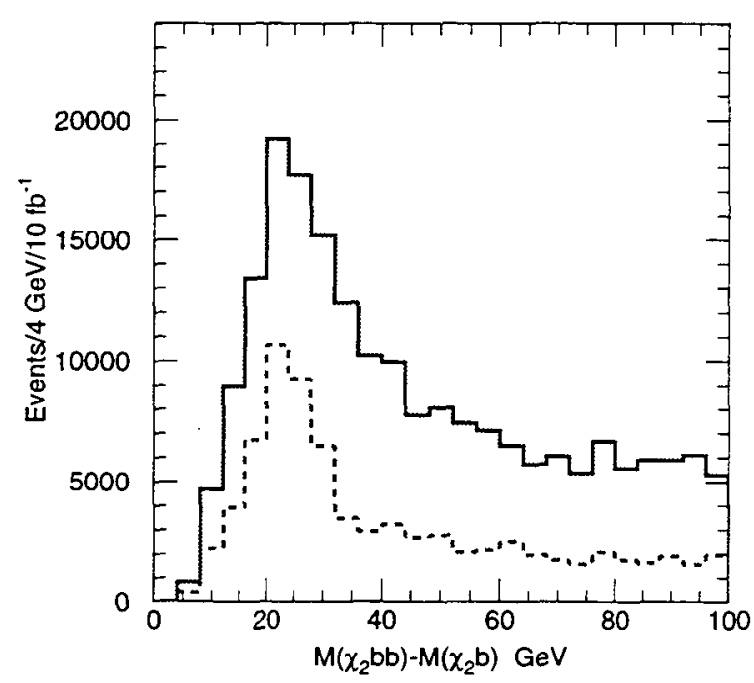

Figure 20-16 $M(\bar{g})-M\left(\tilde{b}_{1}\right)$ projection of Figure 2014. The dashed curve shows the projection with a cut $230 \mathrm{GeV}<M\left(\tilde{b}_{1}\right)<330 \mathrm{GeV}$. 
there is a lot of combinatorial background, the peaks are clearly evident. The statistical errors are negligible, so the errors on the masses are determined by the systematic error on the jet energy scale and the dependence on the $\tilde{\chi}_{1}^{0}$ mass. The difference $M(\tilde{g})-M\left(\tilde{b}_{1}\right)$ is insensitive to the assumed mass, while the reconstructed $\tilde{b}_{1}$ mass peak shifts linearly for events with $M(\tilde{g})-M\left(\tilde{b}_{1}\right)$ within $\pm 15 \mathrm{GeV}$ of its peak value. Combining this with the energy scale uncertainty gives $[20-24]$

$$
\begin{gathered}
\Delta M\left(\tilde{b}_{1}\right)=1.5 \Delta M\left(\tilde{\chi}_{1}^{0}\right) \pm 3 \mathrm{GeV} \\
\Delta\left(M(\tilde{g})-M\left(\tilde{b}_{1}\right)\right)= \pm 2 \mathrm{GeV}
\end{gathered}
$$

where $\Delta M\left(\tilde{\chi}_{1}^{0}\right)$ is the difference between the true and assumed $\tilde{\chi}_{1}^{0}$ masses. At least within the context of the minimal SUGRA model the $\bar{\chi}_{1}^{0}$ mass can be determined accurately by a global fit, as discussed in Section 20.2.9.

Light quarks at Point 3 can be reconstructed in a similar way using the decay $\tilde{q}_{L} \rightarrow \tilde{\chi}_{2}^{0} q$, which has a branching ratio of about $10 \%$. There is an enormous background from $\tilde{g} \rightarrow \tilde{b}_{1} \bar{b}$, so events with $b$-jets must be vetoed. Based on the trade-off between tagging efficiency and light quark rejection found in the $b$-tagging studies documented in Chapter 10 and in the ATLAS Technical Proposal [20-21], this analysis assumed a 90\% veto efficiency of $b$-jets with a $25 \%$ mistagging of light jets as $b$ 's. Events were selected to have

- at least one jet with $p_{\mathrm{T}}>125 \mathrm{GeV}$ and $|\eta|<2$;

- no tagged $b$-jets with $p_{\mathrm{T}}>15 \mathrm{GeV}$ and $|\eta|<2$;

- an opposite-sign, like-flavour $l^{+} l^{-}$pair with $45 \mathrm{GeV}<M_{l l}<55 \mathrm{GeV}$.

The $\tilde{\chi}_{2}^{0}$ momentum was reconstructed as before from the visible dilepton momentum; it was then combined with any jet having $p_{\mathrm{T}}>125 \mathrm{GeV}$ and $|\eta|<2$ to give the mass distribution shown in Figure 20-17. There is a clear signal. Even with a $90 \%$ veto efficiency there is a significant number of $b$-jets remaining in this plot. The signal for true light quark jets as determined from the event generator is shown as the dashed curve in the figure. If the veto efficiency were raised to $95 \%$ using an algorithm optimised for rejection rather than for tagging, then approximately half of this background would be removed, and the peak would shift upward, closer to the mean $\tilde{q}_{L}$ mass of $310 \mathrm{GeV}$. The combinatorial background, shown as the shaded histogram in

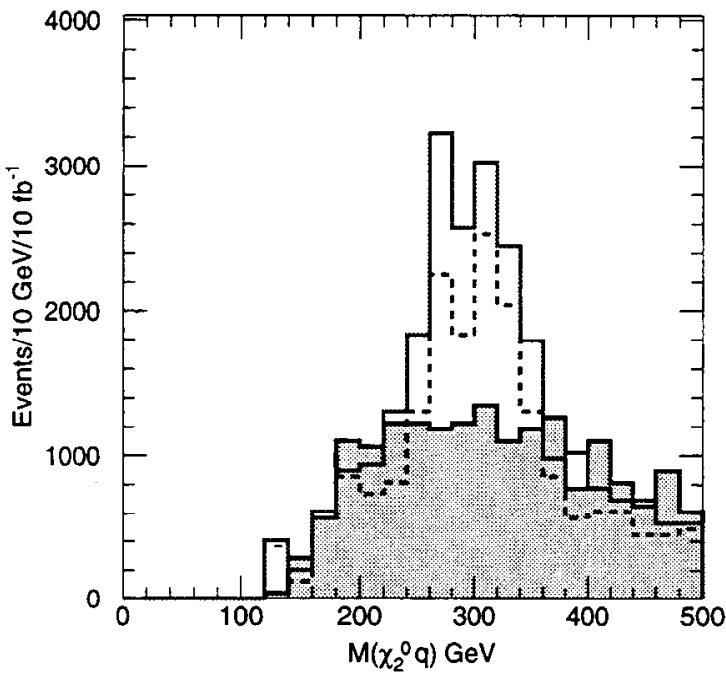

Figure 20-17 Reconstructed $\tilde{q}_{L}$ mass at Point 3. The combinatorial background is shown as a shaded histogram, and the signal due to real $\tilde{q}_{L}$ 's as the dashed histogram. The remaining events are due to misidentified light quark jets.

Figure 20-17, was determined by mixing a $\tilde{\chi}_{2}^{0}$ from one event with a jet from another. Because there is a lot of background, the error on the $\tilde{q}_{L}$ mass is conservatively estimated to be $\pm 20 \mathrm{GeV}$ [20-24]; the purely statistical error would be much less than this. 


\subsubsection{Gluino reconstruction at Point 4}

At Point $4, m_{0}$ is large and the squarks are very heavy, so gluino pair production strongly dominates the SUSY cross section. Nevertheless, reconstructing gluinos is not trivial because of the many competing and complex decay modes. The approach used here is to select events with one gluino decaying via $\tilde{g} \rightarrow q \bar{q}^{\prime} \tilde{\chi}_{\overline{1}}^{ \pm}$and the other via $\tilde{g} \rightarrow q \bar{q} \bar{\chi}_{2}^{0}$ with both gauginos decaying leptonically, giving three leptons and at least four jets. (Two neutralino leptonic decays could also be considered, but this has a smaller branching ratio.) Since the $\tilde{\chi}_{1}^{ \pm}$and $\tilde{\chi}_{2}^{0}$ are almost always nearly degenerate, the mass of the two jets from a single gluino has an endpoint at the difference between the gluino mass and the common gaugino mass. The goal of the analysis is to reconstruct this endpoint.

Events were selected to have:

- three isolated leptons with $p_{\mathrm{T}}>20,10$, and $10 \mathrm{GeV}$ and $|\eta|<2.5$, with at least one opposite-sign, same-flavour pair;

- at least four jets with $p_{\Upsilon}>150,120,70$, and $40 \mathrm{GeV}$ and $|\eta|<2.5$;

- $M_{l l}<72 \mathrm{GeV}$ for any opposite-sign, same-flavour lepton pair to reject $Z$ backgrounds;

- no additional jets with $p_{\mathrm{T}}>40 \mathrm{GeV}$ and $|\eta|<5$ to reduce combinatorial background. This jet veto cut has an efficiency of $35 \%$ for the signal.

The lepton and jet cuts eliminate the Standard Model background, so no $E_{\mathrm{T}}^{\text {miss }}$ cut is used in this analysis. For an integrated luminosity of $30 \mathrm{fb}^{-1}$ there are after these cuts about 250 signal events, 30 SUSY events containing only one gluino, and 18 background events from $t \dot{t}$ and SUSY sources. There are three ways of combining the four jets into two pairs. The pairing of the two hardest and the two softest jets is less likely to be correct and so is excluded. The jet-jet mass distribution for the remaining two combinations is shown in Figure 20-18. This figure also shows the distribution for the correct pairing only, based on information from the generator.

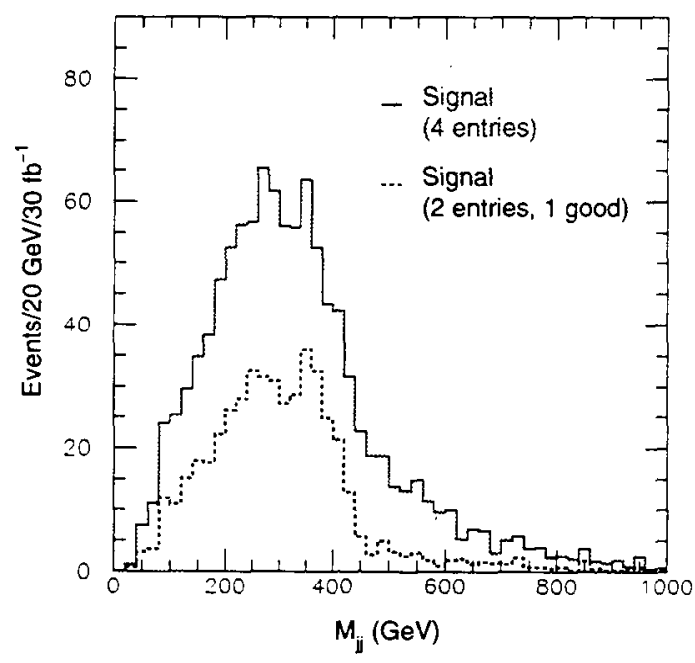

Figure 20-18 Dijet mass distribution for SUSY signal at Point 4 showing all combinations (solid) and only the correct combinations (dashed).

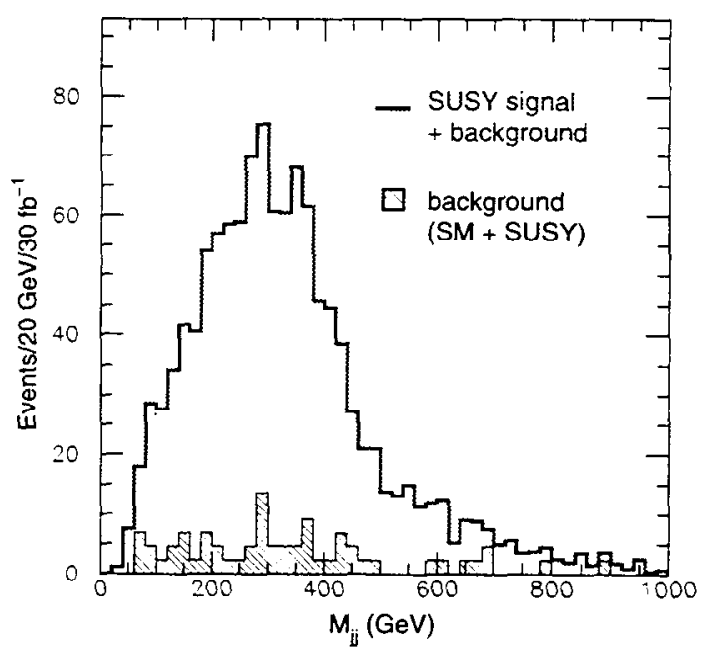

Figure 20-19 Dijet signal and sum of all backgrounds for Point 4. 
The signal plus the sum of all Standard Model backgrounds is shown in Figure 20-19 compared to the background. There is clearly structure in the distribution at about the right mass, but it is not very sharp.

To test the sensitivity of this analysis, events were generated for several different gluino masses keeping all other parameters fixed, and the resulting distributions were compared using a Kolmogorov test. This leads to an estimated error for $30 \mathrm{fb}^{-1}$ of [20-25]

$$
M(\tilde{g})-M\left(\tilde{\chi}_{1} / \tilde{\chi}_{2}^{0}\right)=434_{-16}^{+5.0} \pm 4.5 \mathrm{GeV},
$$

where the first error is statistical and the second is systematic. The statistical error is reduced to $(+1.6,-5.0) \mathrm{GeV}$ for $300 \mathrm{fb}^{-1}$. The systematic error is dominated by the $1 \%$ uncertainty on the jet energy scale.

\subsubsection{Squark reconstruction at Point 5}

At Point 5 gluinos are heavier than squarks and decay into them, so the three-step decay chain $\tilde{q}_{L} \rightarrow \tilde{\chi}_{2}^{0} q \rightarrow l_{R}^{ \pm} l^{\mp} q \rightarrow \tilde{\chi}_{1}^{0} l^{+} l^{-} q$ is the dominant source of the dilepton signal considered in Section 20.2.3. Long decay chains generically give multiple constraints; this one gives constraints from an $l^{ \pm} q$ edge and an $l^{+} l^{-} q$ four-body endpoint in addition to the dilepton endpoint. These provide three constraints on the four masses involved. (Additional constraints are discussed in Sections 20.2.5 and 20.2.6 below.)

The analysis used the same event sample as that which led to Figure 20-12. Events were selected to have [20-29]:

- at least four jets defined with an $R=0.4$ cone, with $p_{\Upsilon, 1}>100 \mathrm{GeV}$ and $p_{\mathrm{T}, 2,3,4}>50 \mathrm{GeV}$;

- $M_{\text {eff }}>400 \mathrm{GeV}$, where $M_{\text {eff }}$ is defined by Equation 20-5;

- $E_{\mathrm{T}}^{\text {miss }}>\max \left(100 \mathrm{GeV}, 0.2 M_{\text {eff }}\right)$;

- two isolated, opposite-sign leptons with $p_{\mathrm{T}}>10 \mathrm{GeV}$ and $|\eta|<2.5$.

In the cascade decays of squarks and gluinos in Point 5 the hardest jets in the event are typically produced in the decays $\tilde{q}_{L} \rightarrow \tilde{\chi}_{2}^{0} q$ and $\tilde{q}_{R} \rightarrow \tilde{\chi}_{1}^{0} q$. The leptons were therefore combined with each of the two hardest jets to ex-

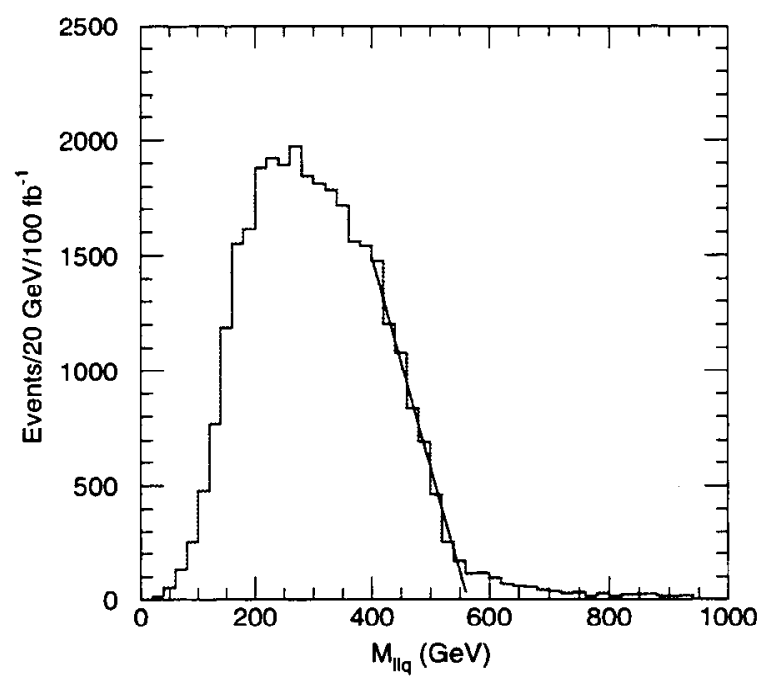

Figure 20-20 Smaller of the two $l^{+} l^{*} q$ masses for the signal at Point 5. tract information on the squark mass. The smaller of the two invariant masses should be less than the four-body endpoint for the $\tilde{q}_{L}$ decay chain, namely

$$
M_{l l q}^{\max }=\left[\frac{\left(M_{\tilde{q}_{L}}^{2}-M_{\tilde{\chi}_{2}^{0}}^{2}\right)\left(M_{\tilde{\chi}_{2}^{0}}^{2}-M_{\tilde{\chi}_{1}^{0}}^{2}\right)}{M_{\tilde{\chi}_{2}^{0}}^{2}}\right]^{1 / 2} \cdot=552.4 \mathrm{GeV}
$$




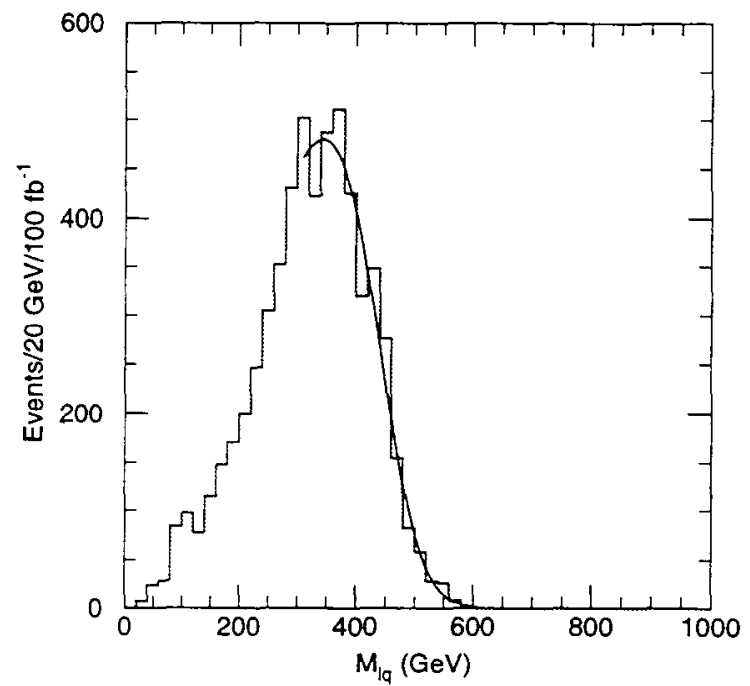

Figure 20-21 $l^{ \pm} q$ mass distribution for combinations with $M_{l^{+} l \cdot q}<600 \mathrm{GeV}$. The smooth curve is a fit to the theoretical form smeared with a Gaussian.

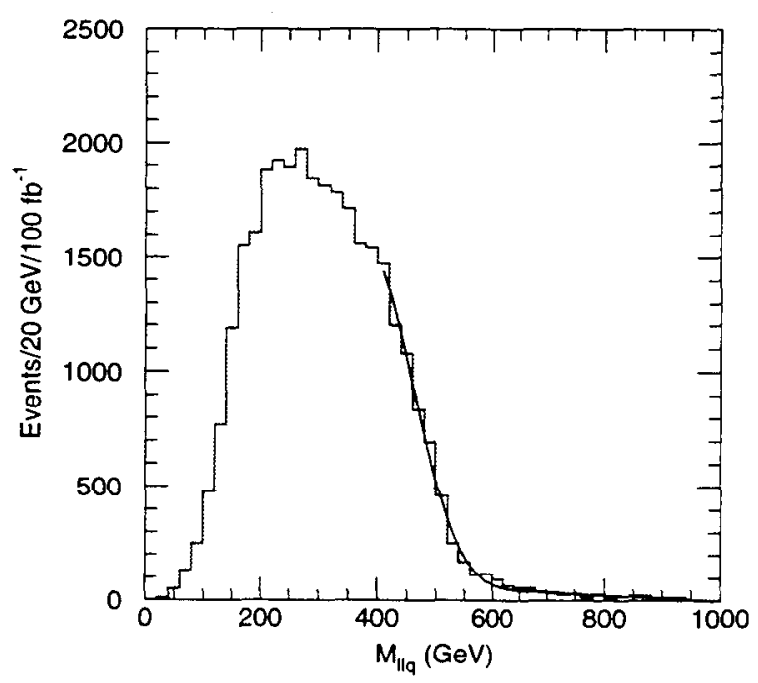

Figure 20-22 Fit described in the text to the smaller of the two $l^{+} l^{-} q$ masses.

The distribution should vanish linearly at this endpoint as a result of the four-body phase space. The distribution of the reconstructed smaller mass is shown in Figure 20-20 for the combination $e^{+} e^{-}+\mu^{+} \mu^{-}-e^{\mp} \mu^{ \pm}$. In this combination the background from two $\chi^{ \pm}$independent decays cancels out. The Standard Model background is not shown but is obviously small, as can be seen from the previous dilepton analysis. The figure also shows a linear fit near the endpoint. It extrapolates to $564.0 \mathrm{GeV}, 2.1 \%$ above the nominal value, providing a first estimate of $M_{l l q}^{\max }$.

The $l l q$ and $l q$ endpoints can be determined more precisely using a fitting procedure that takes into account the smearing due to resolution and jet reconstruction. Events were selected in which one of the $l^{+} l^{-} q$ masses is less than $600 \mathrm{GeV}$ and the other is greater, so that only one pairing of the dilepton pair with one of the two hardest jets is kinematically consistent with the desired decay chain. The cut was chosen to be above the endpoint in Figure 20-20 but is otherwise somewhat arbitrary. The $l^{ \pm} q$ distribution for this subsample, including both lepton-jet pairs from the low-mass $l^{+} l^{-} q$ combination, is shown in Figure 20-21. It should have an edge from the combination of the quark and the 'right' lepton - i.e., the one emitted first and so adjacent to the quark in the decay chain - which from two-body kinematics is

$$
M_{l q}^{\max }=\left[\frac{\left(M_{\bar{q}_{L}}^{2}-M_{\bar{\chi}_{2}^{0}}^{2}\right)\left(M_{\tilde{\chi}_{2}^{0}}^{2}-M_{\tilde{l}_{R}}^{2}\right)}{M_{\bar{\chi}_{2}^{0}}^{2}}\right]^{1 / 2}=479.3 \mathrm{GeV} .
$$

The edge in the figure is not very sharp, so its precise location is not obvious. A fit was therefore made using the theoretical shape of the edge, Equation 20-6 in Section 20.2.3, smeared with a Gaussian resolution. This procedure, which is similar to that for the fit shown in Figure 20-12, assumes that the jet mass can be neglected. The fit, which is shown as the smooth line in Figure 20-21, gives $M_{l q}^{\max }=433.2_{-3.3}^{+3.2} \mathrm{GeV}$, which is $9.6 \%$ low, and $\sigma=58.2 \mathrm{GeV}$. The smearing will also shift the $l^{+} l^{-} q$ endpoint. Figure 20-22 shows a fit using an empirical form with a linear plus a quadratic term near the endpoint,

$$
\frac{d N}{d M}=\left[a_{1}\left(M_{l l q}^{\max }-M\right)+a_{2}\left(M_{l l q}^{\max }-M\right)^{2}\right] \vartheta\left(M_{l l q}^{\max }-M\right),
$$


smeared with the same Gaussian; a linear term was also included in the fit to describe the region beyond the endpoint. This fit produces the smooth curve in Figure 20-22 and $M_{l l q}^{\max }=498.0_{-6.4}^{+7.2} \mathrm{GeV}$, which is $9.9 \%$ low. Presumably the low values reflect energy loss out of the small jet cones, $R=0.4$, and the neglect of the jet masses. To understand the shifts and the associated errors in detail, it would be necessary to generate and to analyse a number of different samples of events. This has not been done.

The ratio of the $l^{ \pm} q$ and $l^{+} l^{-} q$ endpoints is independent of the squark mass,

$$
\frac{M_{l q}^{\max }}{M_{l q}^{\max }}=\sqrt{\frac{M_{\tilde{\chi}_{2}^{0}}^{2}-M_{\hat{l}_{R}}^{2}}{M_{\tilde{\chi}_{2}^{0}}^{2}-M_{\tilde{\chi}_{1}^{0}}^{2}}}=0.868,
$$

and it should be less sensitive to the jet energy scale and to jet clustering than either of the individual endpoints. The smeared fits give 0.870 for this ratio. This probably is fortuitously good agreement, although an analysis of the same data sample using jets defined with $R=0.7$ gave an equally good result. More study of the systematic errors from the fitting procedure is needed.

There is also a linear vanishing of the distribution for the $l^{ \pm} q$ mass combination formed with the 'wrong' lepton and the jet at

$$
M_{l q}^{\max }=\left[\frac{\left(M_{\tilde{q}_{L}}^{2}-M_{\tilde{\chi}_{2}^{0}}^{2}\right)\left(M_{\bar{l}_{R}}^{2}-M_{\tilde{\chi}_{1}^{0}}^{2}\right)}{M_{\tilde{\chi}_{2}^{0}}^{2}}\right]^{1 / 2}=274.5 \mathrm{GeV}
$$

Since this endpoint is below the edge of the distribution with the right lepton, it is not usable; an analogous endpoint is used for the GMSB model studied in Section 20.3.1.

\subsection{5 $h \rightarrow b \bar{b}$ SUGRA signatures}

If the two-body decay $\tilde{\chi}_{2}^{0} \rightarrow \bar{\chi}_{1}^{0} h$ is kinematically allowed, it generally has a substantial branching ratio because the light neutralinos are dominantly gauginos. In many cases it is possible to reconstruct $h \rightarrow b \bar{b}$ as a resonance peak in the SUSY event sample. This signal may be easier to detect than $h \rightarrow \gamma \gamma$ and so provide the discovery mode for the light Higgs boson, although the $\gamma \gamma$ signal is still important to measure the mass precisely. The Higgs signal can also provide a good starting point for further analysis of SUSY particles. Points 1, 2 and 5 all have substantial $\tilde{\chi}_{2}^{0} \rightarrow \tilde{\chi}_{1}^{0} h$ decays that can be reconstructed and used in this way.

At Point 1 the branching ratio for $\tilde{\chi}_{2}^{0} \rightarrow \tilde{\chi}_{1}^{0} h$ is near unity; since the light neutralinos are mainly gauginos, the branching ratio for the only other two-body decay, $\tilde{\chi}_{2}^{0} \rightarrow \tilde{\chi}_{1}^{0} Z$, is small. Hence, about $20 \%$ of SUSY events contain at least one $h \rightarrow b \bar{b}$. The following cuts were chosen [20-29] to select the signal and eliminate Standard Model backgrounds:

- $E_{\mathrm{T}}^{\text {miss }}>300 \mathrm{GeV}$;

- exactly two tagged $b$-jets with $p_{\mathrm{T}}>50 \mathrm{GeV}$ (as usual a $b$-tagging efficiency of $60 \%$ is used);

- $\Delta R_{b b}<2$ to help suppress $t \dot{t}$ background;

- a lepton veto, also to help suppress $t \dot{t}$ background;

- at least two additional jets with $p_{\mathrm{T}}>100 \mathrm{GeV}$, at least one of which has $|\eta|<2$. 


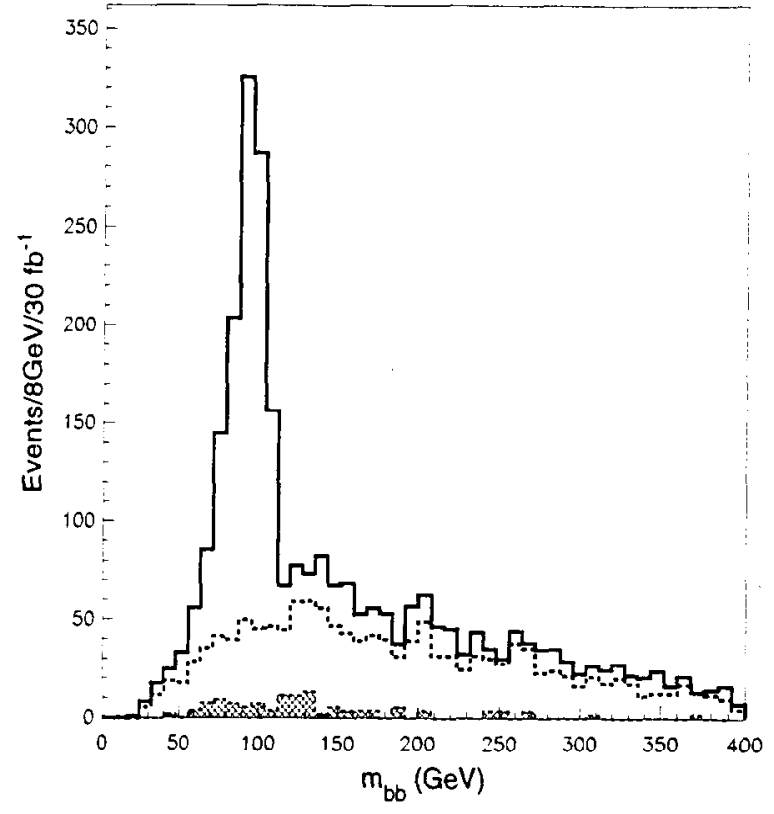

Figure 20-23 Mass distribution for two tagged $b$-jets at Point 1 for $30 \mathrm{fb}^{-1}$. The dotted curve is the SUSY background, and the shaded histogram is the sum of all Standard Model backgrounds.
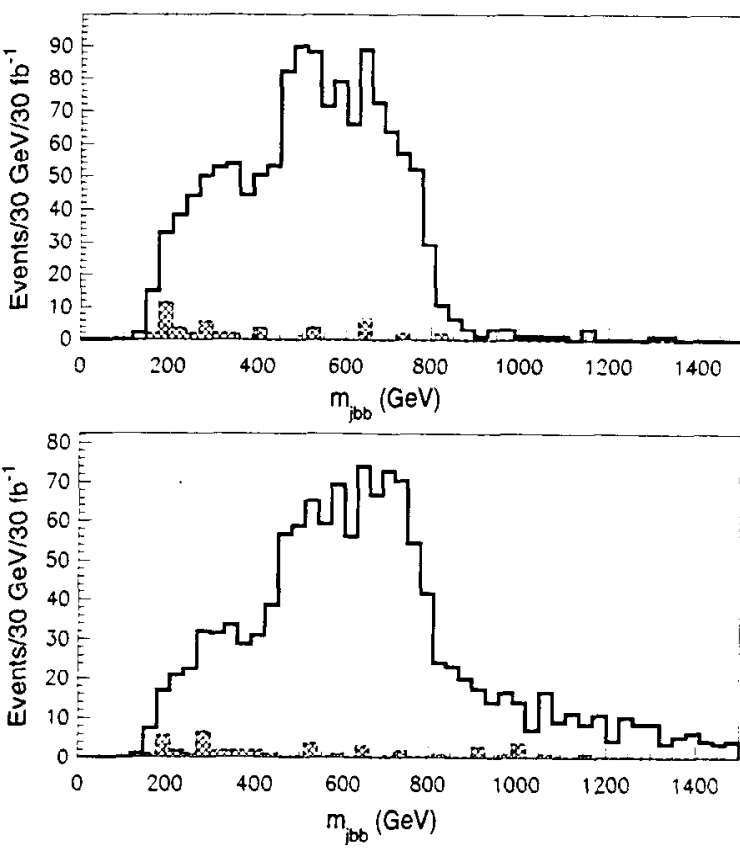

Figure 20-24 $b b j$ mass distribution for events within $\pm 25 \mathrm{GeV}$ of the peak in Figure $20-23$. Top plot: combination with smaller mass. Bottom plot: all combinations.

With these cuts the signal to background ratio is very good, as can be seen in Figure 20-23. For an integrated luminosity of $30 \mathrm{fb}^{-1}$ there are 980 signal events with a SUSY background of 180 events and a Standard Model background of only 75 events in a bin of $\pm 25 \mathrm{GeV}$ around the Higgs mass. Given this rate it would be much easier to discover the light Higgs by selecting SUSY events than in the inclusive $h \rightarrow r \gamma$ mode.

Since the gluino is heavier than the squarks at this point, the dominant source of the signal is the decay chain $\tilde{q}_{L} \rightarrow \tilde{\chi}_{2}^{0} q \rightarrow \tilde{\chi}_{1}^{0} h q$, in which the squark may be produced either directly or from a gluino. For this decay chain there is a maximum value of the $h q$ invariant mass that is determined by two-body kinematics [20-22]:

$$
\left(M_{h q}^{\max }\right)^{2}=M_{h}^{2}+\left(M_{\tilde{q}}^{2}-M_{\tilde{\chi}_{2}^{0}}^{2}\right)\left[\frac{M_{\tilde{\chi}_{2}^{0}}^{2}+M_{h}^{2}-M_{\chi_{1}^{0}}^{2}+\sqrt{\left(M_{\tilde{\chi}_{2}^{0}}^{2}-M_{h}^{2}-M_{\tilde{\chi}_{1}^{0}}^{2}\right)^{2}-4 M_{h}^{2} M_{\tilde{\chi}_{1}^{0}}^{2}}}{2 M_{\tilde{\chi}_{2}^{0}}^{2}}\right] .
$$

About $70 \%$ of the signal comes from $\tilde{q}_{L} \tilde{q}_{R}$ production, either directly or from gluino decays, with $\tilde{q}_{L} \rightarrow \tilde{\chi}_{2}^{0} q$ and $\tilde{q}_{R} \rightarrow \tilde{\chi}_{1}^{0} q$. Hence, it is advantageous to obtain a cleaner sample by selecting events with only two hard jets in addition to the pair of $b$-jets within $\pm 25 \mathrm{GeV}$ of the peak, vetoing events having any additional jets with $p_{T}>50 \mathrm{GeV}$ and $|\eta|<5$. Then the hard jets are likely to come from the squarks, so the smaller of the two $b \bar{b} q$ masses should be less than the endpoint for squark decay. As can be seen from Figure 20-24, the distribution for the smaller mass has a much better defined endpoint than that including both combinations. The estimated error [20-29] on this endpoint is $\pm 30 \mathrm{GeV}$ for $30 \mathrm{fb}^{-1}$. The improvement in the error for three years at high luminosity is estimated to be about a factor of two, limited both by the jet energy scale and by the reduced $b$-tagging efficiency at high luminosity. 

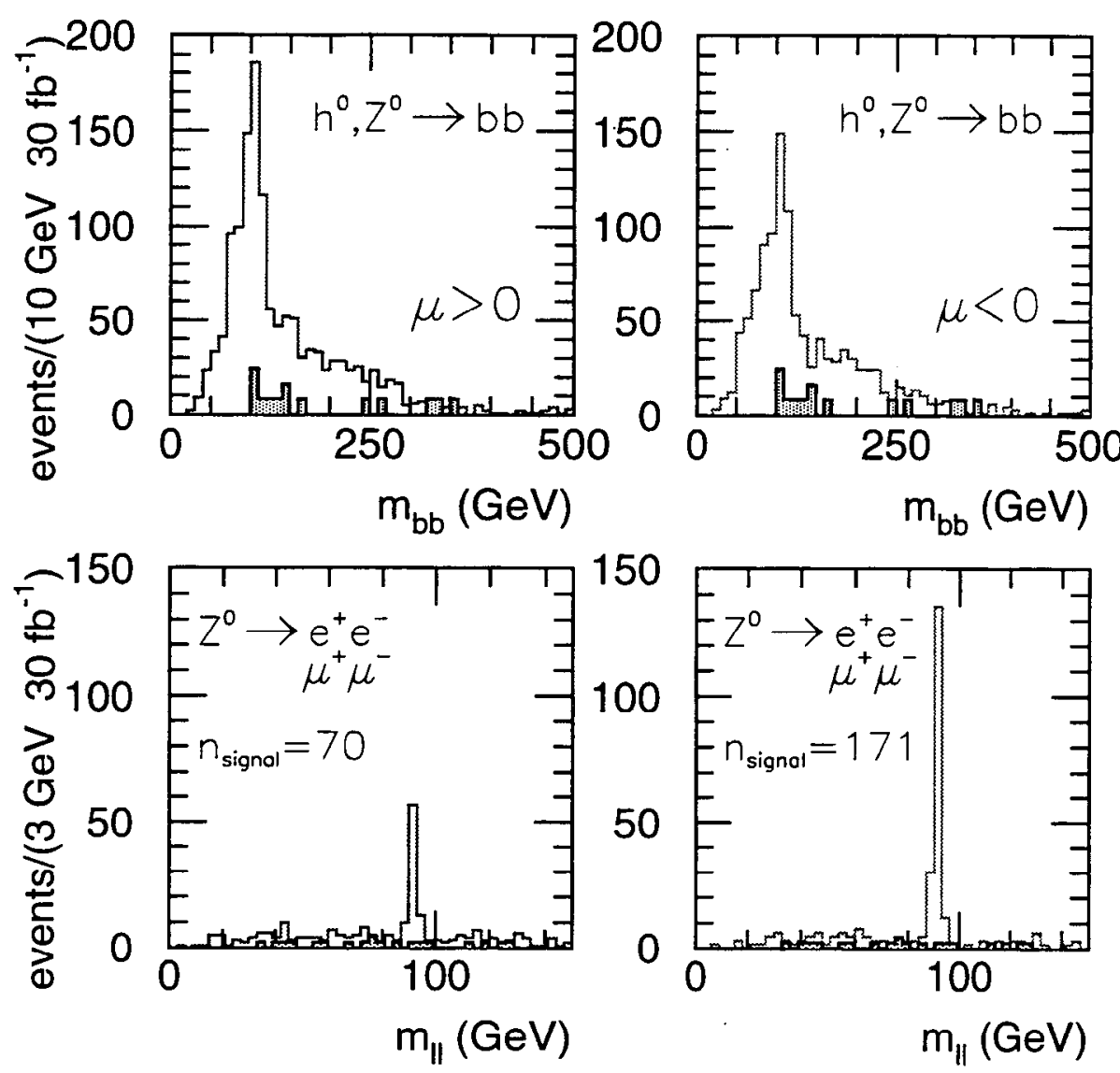

Figure 20-25 $b \bar{b}$ dijet and dilepton mass distributions for Point 2 and for a point with same parameters except $\mu<0$. The shaded distributions show the Standard Model backgrounds.

For Point 2 with $\tan \beta=10$ the light gauginos have more Higgsino content. Hence the branching ratio for $\tilde{\chi}_{2}^{0} \rightarrow \tilde{\chi}_{1}^{0} h$ is about $65 \%$, with the rest of its decays being mainly $\tilde{\chi}_{2}^{0} \rightarrow \tilde{\chi}_{1}^{0} Z$. The $\tilde{\chi}_{2}^{0} \rightarrow \tilde{\chi}_{1}^{0} h$ branching ratio for the same parameters with $\operatorname{sgn} \mu=-1$ is about $91 \%$. The dijet mass resolution is not good enough to separate the $\tilde{\chi}_{2}^{0} \rightarrow \bar{\chi}_{1}^{0} h$ and $\tilde{\chi}_{2}^{0} \rightarrow \tilde{\chi}_{1}^{0} Z$ decays, so it is important to study the $Z \rightarrow l^{+} l^{-}$signal as well. For the $h \rightarrow b \bar{b}$ signal events are selected to have [20-23]

- $E_{\mathrm{T}}^{\mathrm{miss}}>300 \mathrm{GeV}$;

- at least two jets not tagged as $b$ 's with $p_{\mathrm{T}}>100 \mathrm{GeV}$;

- two tagged $b$-jets with $p_{\mathrm{T}}>100 \mathrm{GeV}$;

- $\Delta R_{b h}<1.0$.

For the dilepton sample the cuts are

- $E_{\mathrm{T}}^{\mathrm{miss}}>300 \mathrm{GeV}$;

- at least two jets not tagged as $b$ 's with $p_{\mathrm{T}}>100 \mathrm{GeV}$;

- two opposite-sign, same flavour leptons with $p_{\mathrm{T}}>10 \mathrm{GeV}$. 


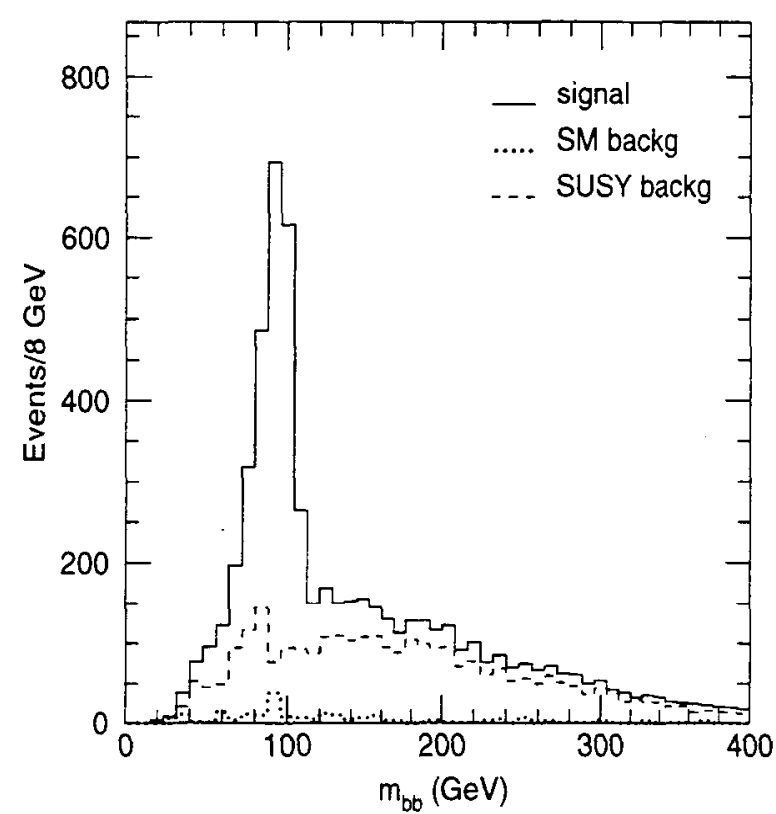

Figure 20-26 Corrected mass distribution for two $b$ jets for Point 5 Higgs SUSY signal (solid), SUSY background (dashed) and Standard Model background (dotted).

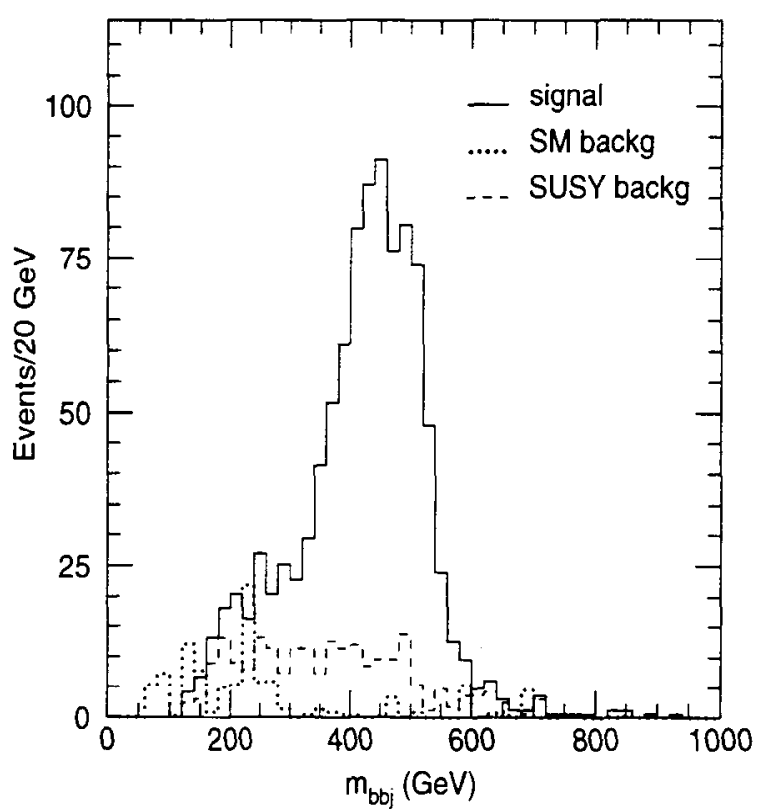

Figure 20-27 Smaller $b b q$ mass for events in the Higgs peak in Figure 20-26.

The resulting dijet and dilepton mass distributions are shown in Figure 20-25 for both signs of $\mu$. Even after correction for the kinematic cuts and for efficiencies these distributions do not directly measure the $\tilde{\chi}_{2}^{0}$ branching ratios, since there is a non-negligible contribution to the $Z$ signals from $\tilde{\chi}_{2}^{ \pm} \rightarrow \bar{\chi}_{1}^{ \pm} Z$. Hence, while the measurement is clearly useful, it is not easy to translate into limits on the SUSY model without a more detailed analysis.

The $h \rightarrow b \bar{b}$ analysis at Point 5 is quite similar to that at Point 1 . The following selection cuts are made to select the signal and reject both SUSY and Standard Model backgrounds [20-26]:

- $E_{\mathrm{T}}^{\mathrm{miss}}>300 \mathrm{GeV}$;

- two tagged $b$-jets with $p_{\mathrm{T}}>50 \mathrm{GeV}$;

- no additional $b$-jets with $p_{\mathrm{T}}>15 \mathrm{GeV}$;

- at least two jets with $p_{\mathrm{T}}>100 \mathrm{GeV}$;

- no isolated leptons with $p_{\mathrm{T}}>10 \mathrm{GeV}$.

The signal and backgrounds after these cuts are shown in Figure 20-26. The event and detector Monte Carlos were used to derive a correction to the $b$-jet energy scale for losses from energy out of the cone used to define the jets and from neutrinos. With this correction, the mass of the Higgs can be measured with an estimated error of $1 \mathrm{GeV}$ from this distribution with $30 \mathrm{fb}^{-1}$ of luminosity. Again, it is easier to discover the Higgs in this channel than in $h \rightarrow \gamma \gamma$, although the latter will provide a much more accurate mass.

The Higgs signal comes from a mixture of gluino and squark production, but in most cases the two hardest jets come from the squark decays whether they are produced directly or in gluino decays. Therefore, the smaller of the two masses formed from the Higgs plus one of these two hardest jets should have an endpoint given by Equation 20-8. This distribution is shown in 
Figure 20-27 and indeed exhibits the expected endpoint. If the remaining masses in Equation 20-8 are known from other measurements, then the estimated error on the $\tilde{q}_{L}$ mass is $\pm 10 \mathrm{GeV}$ for $30 \mathrm{fb}^{-1}$, dominated by the jet energy scale [20-26].

\subsubsection{Thresholds and model-independent SUGRA masses}

In multi-step decays such as $\tilde{q}_{L} \rightarrow \tilde{\chi}_{2}^{0} q, \tilde{\chi}_{2}^{0} \rightarrow \tilde{\chi}_{1}^{0} h$ or $\tilde{q}_{L} \rightarrow \tilde{\chi}_{2}^{0} q, \tilde{\chi}_{2}^{0} \rightarrow \tilde{\chi}_{1}^{0} l l$, there is in general both a maximum and a minimum value of the observable mass as a consequence of two-body kinematics. The minimum value comes from a backwards decay of the second SUSY particle in the centre of mass of the first and can provide a measurable 'threshold' that determines a different combination of masses than the edges discussed previously [20-29]. This is analogous to the method proposed to measure SUSY masses at $e^{+} e^{-}$machines [20-32]. For $e^{+} e^{-} \rightarrow \tilde{\mu}^{+} \tilde{\mu}^{-} \rightarrow \tilde{\chi}_{1}^{0} \mu^{+} \tilde{\chi}_{1}^{0} \mu^{-}$at a fixed centre-of-mass energy, the maximum and minimum values of the muon energy determine the $\tilde{\mu}$ and $\tilde{\chi}_{1}^{0}$ masses. For the LHC case, the squark mass plays the role of the initial energy, and invariant masses rather than laboratory energies must be used, but the basic idea is the same. At Point 5 the $l^{+} l^{-} q$ threshold can be measured and combined with the results discussed previously to determine the $\tilde{q}_{L}, \tilde{\chi}_{2}^{0}, \tilde{l}_{R}$, and $\tilde{\chi}_{1}^{0}$ masses using only kinematics rather than relying on a fit to the minimal SUGRA as in Section 20.2 .9 below. In this sense the determination of the masses is model independent; the feasibility of doing such an analysis is of course very model dependent.

At Point 5, the dilepton mass from $\tilde{\chi}_{2}^{0} \rightarrow \tilde{l}_{R}^{ \pm} l^{\mp} \rightarrow \tilde{\chi}_{1}^{0} l^{+} l$ is fixed by the $\tilde{\chi}_{2}^{0}, \tilde{l}_{R}$, and $\tilde{\chi}_{1}^{0}$ masses and by $z=\cos \theta^{*}$, where $\theta^{*}$ is the decay angle in the $\tilde{l}_{R}^{ \pm}$centre of mass relative to the $I^{F}$ direction. These quantities also determine the dilepton momentum in the $\tilde{\chi}_{2}^{0}$ centre of mass. If this momentum is not too large - i.e., if the minimum dilepton mass is not too small - then there is obviously a minimum $l l q$ mass from the $\tilde{q}_{L} \rightarrow \tilde{\chi}_{2}^{0} q \rightarrow l_{R}^{t} l^{\mp} q \rightarrow \tilde{\chi}_{1}^{0} l^{+} l^{-} q$ decay chain. The general expression is rather complicated but simplifies somewhat for $z=0$ :

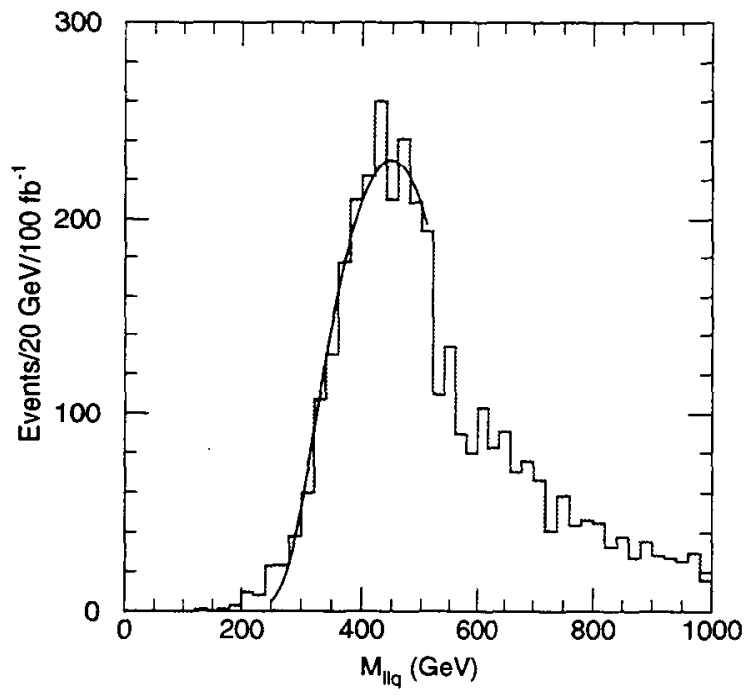

Figure 20-28 Distribution of the larger $l l q$ mass at Point 5 for $M_{l l}>M_{l l}^{\max } / \sqrt{2}$ and a fit described in the text.

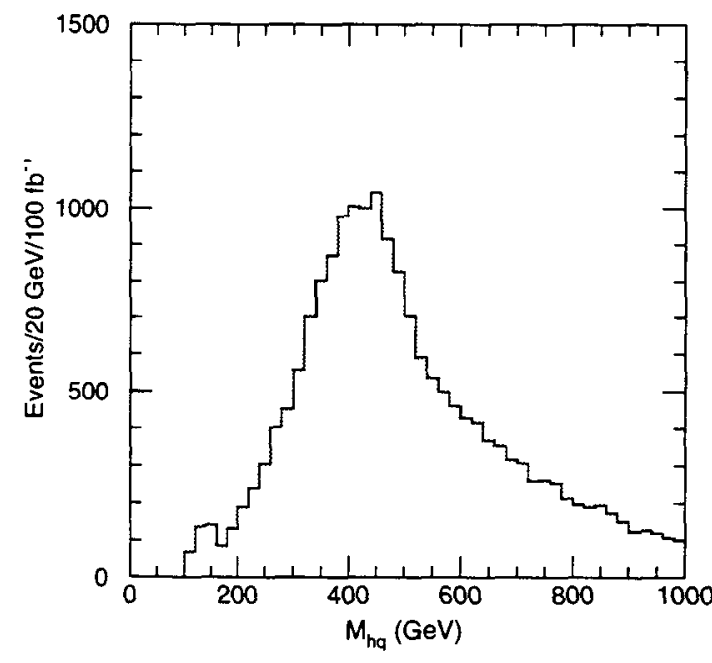

Figure 20-29 Distribution of the larger $b b q$ mass at Point 5. No sideband subtraction or $b$-jet energy correction has been made. 


$$
\begin{aligned}
\left(M_{l l q}^{\min }\right)^{2}=\frac{1}{4 M_{2}^{2} M_{e}^{2}}[ & -M_{1}^{2} M_{2}^{4}+3 M_{1}^{2} M_{2}^{2} M_{e}^{2}-M_{2}^{4} M_{e}^{2}-M_{2}^{2} M_{e}^{4}-M_{1}^{2} M_{2}^{2} M_{q}^{2} \\
& -M_{1}^{2} M_{e}^{2} M_{q}^{2}+3 M_{2}^{2} M_{e}^{2} M_{q}^{2}-M_{e}^{4} M_{q}^{2}+\left(M_{2}^{2}-M_{q}^{2}\right) \\
& \left.\times \sqrt{\left(M_{1}^{4}+M_{e}^{4}\right)\left(M_{2}^{2}+M_{e}^{2}\right)^{2}+2 M_{1}^{2} M_{e}^{2}\left(M_{2}^{4}-6 M_{2}^{2} M_{2}^{2} M_{e}^{2}+M_{e}^{4}\right)}\right]
\end{aligned}
$$

where the shorthand notation $M_{q}=M_{\tilde{q}_{L}}, M_{2}=M_{\tilde{\chi}_{2}^{0}}, M_{e}=M_{\tilde{e}_{R}}=M_{\tilde{\mu}_{R}}$, and $M_{1}=M_{\tilde{\chi}_{1}^{0}}$ has been used and where all quark and lepton masses have been neglected. For the masses at Point $5, M_{l l q}^{\min }=271.8 \mathrm{GeV}$.

To extract this threshold, the same sample of events was used as for Figure 20-12. Events were selected as before with the additional requirement $M_{l l}>M_{l l}^{\max } / \sqrt{2}$, corresponding to $z>0$. The cross section is dominated by squark pairs produced either directly or from gluino decay. Therefore, the larger of the two $l l q$ masses formed from the dilepton pair and one of the two highest $p_{T}$ jets was selected; since the hardest jets presumably come directly from the squark, at least one of the two combinations should be greater than the minimum for squark decay. To extract the position of the edge, a fit was then made using the empirical functional form

$$
\left[b_{1}\left(M-M_{l l q}^{\min }\right)+b_{2}\left(M-M_{l l q}^{\min }\right)^{2}\right] \vartheta\left(M-M_{l l q}^{\min }\right)
$$

smeared with a Gaussian resolution. The parameters are the desired $M_{l l y}^{\min }$, the width of the Gaussian resolution, and the coefficients $b_{1,2}$. If the width was left free in the fit, then the result was quite sensitive to the interval included, so the width was constrained to be $10 \%$ of $M_{l l q}^{\min }$, similar to what was found before. The distribution for $100 \mathrm{fb}^{-1}$ and the resulting fit are shown in Figure 20-28. The fit with MINUIT and MINOS gave $M_{l l q}^{\min }=283.7_{-4.5}^{+4.4} \mathrm{GeV}$, a statistical error of about $\pm 1.5 \%$. The $\chi^{2}$ of the fit is poor mainly because of the few bins near $200 \mathrm{GeV}$; these bins presumably also are responsible for the instability of the fit when the width was left free. Clearly more study is needed to understand the shape of this distribution and the optimal fitting procedure, but it seems plausible that this edge could be measured to $\pm 2 \%$.

Two-body kinematics implies that there is also a threshold for the $h q$ mass from $\tilde{q}_{L} \rightarrow \tilde{\chi}_{2}^{0} q \rightarrow \tilde{\chi}_{1}^{0} h q$ at

$$
\left(M_{h q}^{\min }\right)^{2}=\frac{\left(M_{q}^{2}-M_{2}^{2}\right)}{2 M_{2}^{2}}\left[M_{2}^{2}+M_{h}^{2}-M_{1}^{2}-\sqrt{\left(M_{2}^{2}-M_{h}^{2}-M_{1}^{2}\right)^{2}-4 M_{1}^{2} M_{h}^{2}}\right],
$$

where the same shorthand notation as before is used. For Point 5, $M_{h q}^{\min }=271.8 \mathrm{GeV}$. Events were selected having exactly two $b$-jets with $70<M_{b b}<110 \mathrm{GeV}$, and the larger of the two $b b j$ masses formed by these and one of the two hardest jets in the event was taken. This distribution is shown in Figure 20-29 with no correction for the $b$-jet energy scale or sideband subtraction. Clearly this analysis could be improved, but it is unlikely to be as precise as the $l l q$ threshold. For now it is included in the analysis with a large error, $\pm 5 \%$.

For Point 5, although the $l^{+} l^{-}$edge, $l^{ \pm} q$ edge, $l^{+} l^{*} q$ 4-body endpoint, and $h q$ edge described earlier give four constraints on the $\tilde{q}_{L}, \tilde{\chi}_{2}^{0}, \tilde{l}_{R}$, and $\tilde{\chi}_{1}^{0}$ masses, it turns out that there exists a solution for any value of the $\tilde{\chi}_{1}^{0}$ mass. Including the $l^{+} l^{-} q$ threshold described here makes it possible to determine all the masses involved from kinematics alone. The errors for $100 \mathrm{fb}^{-1}$ of luminosity are assumed to be $1 \%$ on the $l^{ \pm} q$ and $l^{+} l^{-} q$ edges, $2 \%$ on the $l^{+} l^{-} q$ threshold, and negligible on the $l^{+} l^{-}$edge. These constraints were solved numerically: the $\tilde{q}_{L}, \tilde{\chi}_{2}^{0}$, and $\tilde{l}_{R}$ masses were generated with uniform random distributions within $\pm 50 \%$ of their input values, and the $\tilde{\chi}_{1}^{0}$ mass was computed from these and the $l^{+} l^{-}$edge. The measured quantities were then 


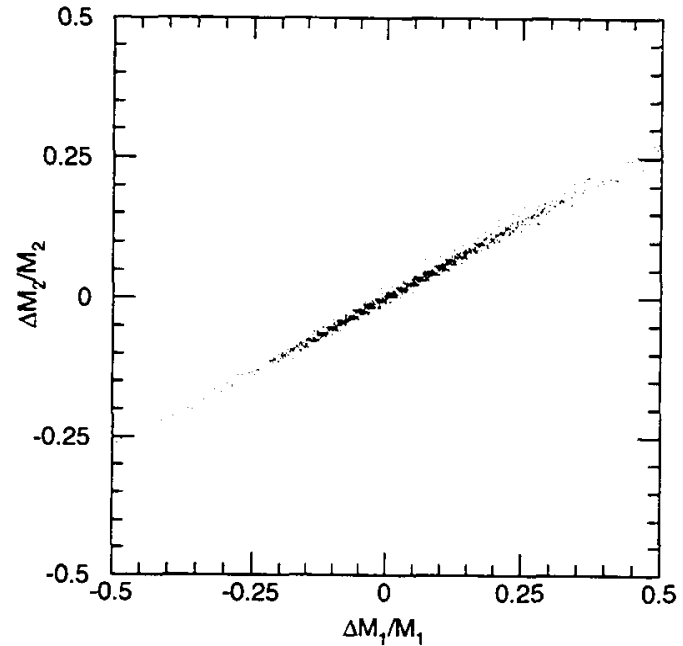

Figure 20-30 $\Delta M_{\bar{\chi}_{2}^{0}} / M_{\bar{\chi}_{2}^{0}}$ as a function of $\Delta M_{\tilde{x}^{0}} / M_{\tilde{\chi}^{0}}$ for masses from model-independent analy'sis for 'Point 5.

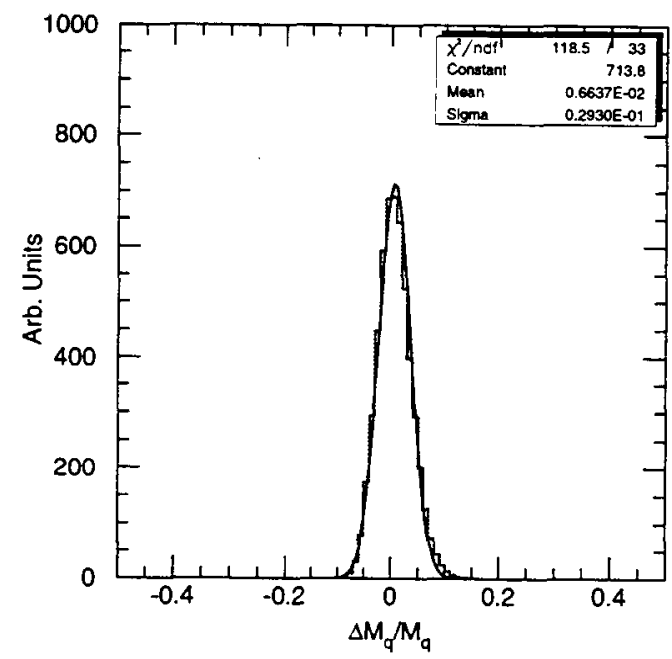

Figure 20-32 Distribution of the fractional difference between the reconstructed and the true squark mass from model-independent analysis for Point 5.

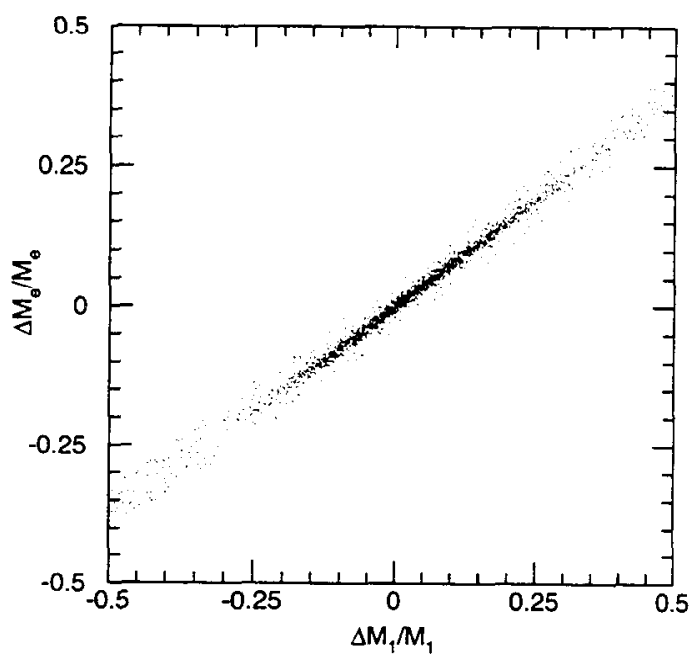

Figure 20-31 $\Delta M_{\tilde{l}_{R}} / M_{\tilde{l}_{z}}$ as function of $\Delta M_{\tilde{\chi}^{0}} / M_{\tilde{\chi}^{0}}$ for masses from ${ }^{l}$ modêl-independent analysis for Point 5.

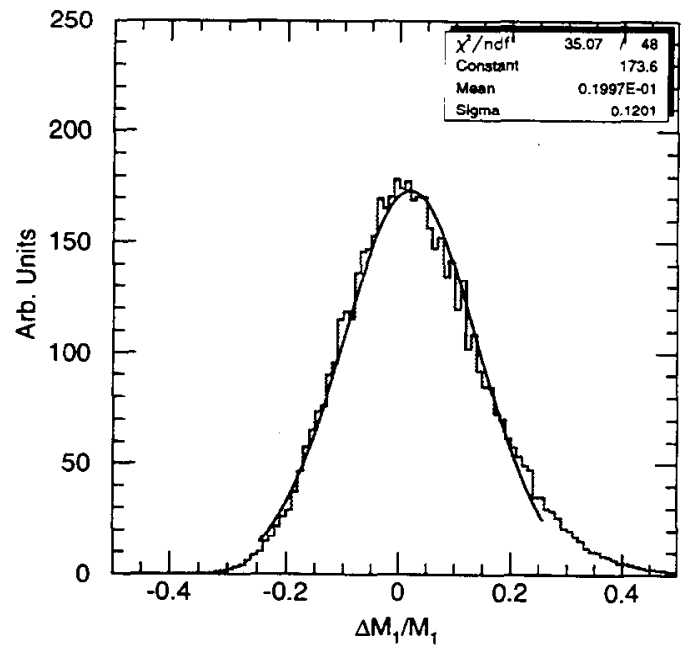

Figure 20-33 Distribution of the fractional difference between the reconstructed and the true $\tilde{\chi}_{1}^{0}$ mass from model-independent analysis for Point 5.

calculated, and the point was weighted using the assumed errors with Gaussian distributions. The resulting masses [20-29] are shown as scatter plots in Figures 20-30 and 20-31. Clearly the allowed masses are highly correlated. The width of the distributions of the difference between true and reconstructed mass is about $\pm 3 \%$ for the squark mass, shown in Figure $20-32, \pm 6 \%$ for the $\tilde{\chi}_{2}^{0}$ mass, $\pm 9 \%$ for the slepton mass, and $\pm 12 \%$ for the $\tilde{\chi}_{1}^{0}$ mass, shown in Figure $20-33$. The widths of these distributions are mainly controlled by the error on the $l^{+} l^{-} q$ threshold. If this constraint were not included, the $\tilde{\chi}_{1}^{0}$ mass would be essentially undetermined. If this error could be reduced to $\pm 1 \%$ with a more sophisticated fitting procedure and several years at full luminosity, the error on the $\tilde{\chi}_{1}^{0}$ mass would be reduced to about $\pm 7 \%$. 
The errors obtained here are of course larger than those found in Section 20.2 .9 below by fitting the minimal SUGRA model to the same measurements, but this analysis is done without assuming that the underlying model is known and would still be possible even if the model has a large number of parameters. The interpretations that $\tilde{q}_{L}$ rather than $\tilde{q}_{R}$ is the main source of $\tilde{\chi}_{2}^{0}$ and the lighter slepton is a $\tilde{l}_{R}$ rather than an $\tilde{l}_{L}$ are of course model dependent assumptions, although ones that are much more general than the minimal SUGRA model. While the specific results only apply to this particular point, any long sequence of decays like $\tilde{q}_{L} \rightarrow \tilde{\chi}_{2}^{0} q \rightarrow \tilde{l}^{ \pm} l^{\mp} q \rightarrow \tilde{\chi}_{1}^{0} l^{+} l^{-} q$ will in general give multiple constraints that potentially allow the individual masses to be determined through the measurement of several kinematic endpoints. Another example of the

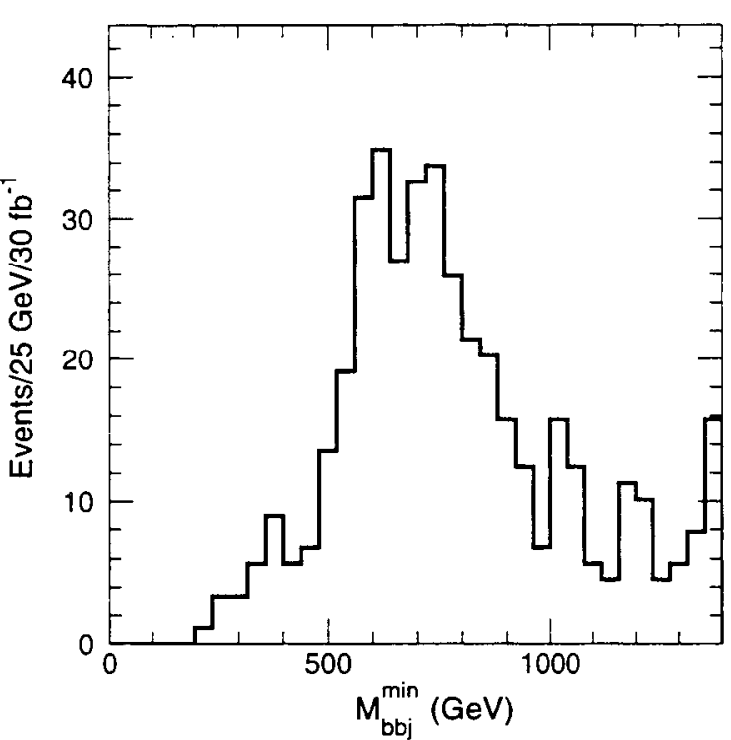

Figure 20-34 Distribution of the larger $b b q$ mass at Point 1after rescaling the $b$-jets to $M_{h}$. power of identifying long decay chains is presented for one of the GMSB points in Section 20.3.3.1.

For Point 1 there is no dilepton signature, so the $h q$ threshold, located at $M_{h q}^{\min }=325.2 \mathrm{GeV}$, is more useful. Events were selected with two $b$-jets near the Higgs mass, two hard jets, and no other jets with $p_{\mathrm{T}}>100 \mathrm{GeV}$ to reduce the combinatorial background. The momenta of the $b$ jets were then rescaled to give the correct Higgs mass, and the larger of the two masses formed by these and one of the hard jets was selected. This distribution is shown in Figure 20-34. More study is needed to understand how to extract the edge from this distribution, but it clearly contains useful information.

\subsubsection{Other signatures for SUGRA Points $1-5$}

While signatures based either on $\tilde{\chi}_{2}^{0} \rightarrow \tilde{\chi}_{1}^{0} l^{+} l^{-}$or on $\tilde{\chi}_{2}^{0} \rightarrow \tilde{\chi}_{1}^{0} h$ are rather generally applicable, other exclusive modes can be reconstructed in special cases. This subsection contains a number of examples for the five LHCC points listed in Table 20-3. In some cases masses have been extracted by using the shapes of distributions and not just kinematic endpoints.

\subsubsection{1 $q_{R} \rightarrow \tilde{\chi}_{1}^{0} q$ signature at Point 1}

At Points 1 and 2 the branching ratio for $q_{R} \rightarrow \tilde{\chi}_{1}^{0} q$ is near unity, so production of $\tilde{q}_{R}$ pairs gives rise to events with two hard jets, no other jets except those from $Q C D$ radiation, and large missing transverse energy. The following cuts were made [20-23] to extract the signal and reject Standard Model backgrounds:

- $E_{\mathrm{T}}^{\text {miss }}>400 \mathrm{GeV}$;

- two hard central jets with $p_{\mathrm{T}}>200 \mathrm{GeV}$ and $|\eta|<2$;

- $p_{\mathrm{T}, 1}+p_{\mathrm{T}, 2}>500 \mathrm{GeV}$;

- $\Delta R>1$ between the two jets; 

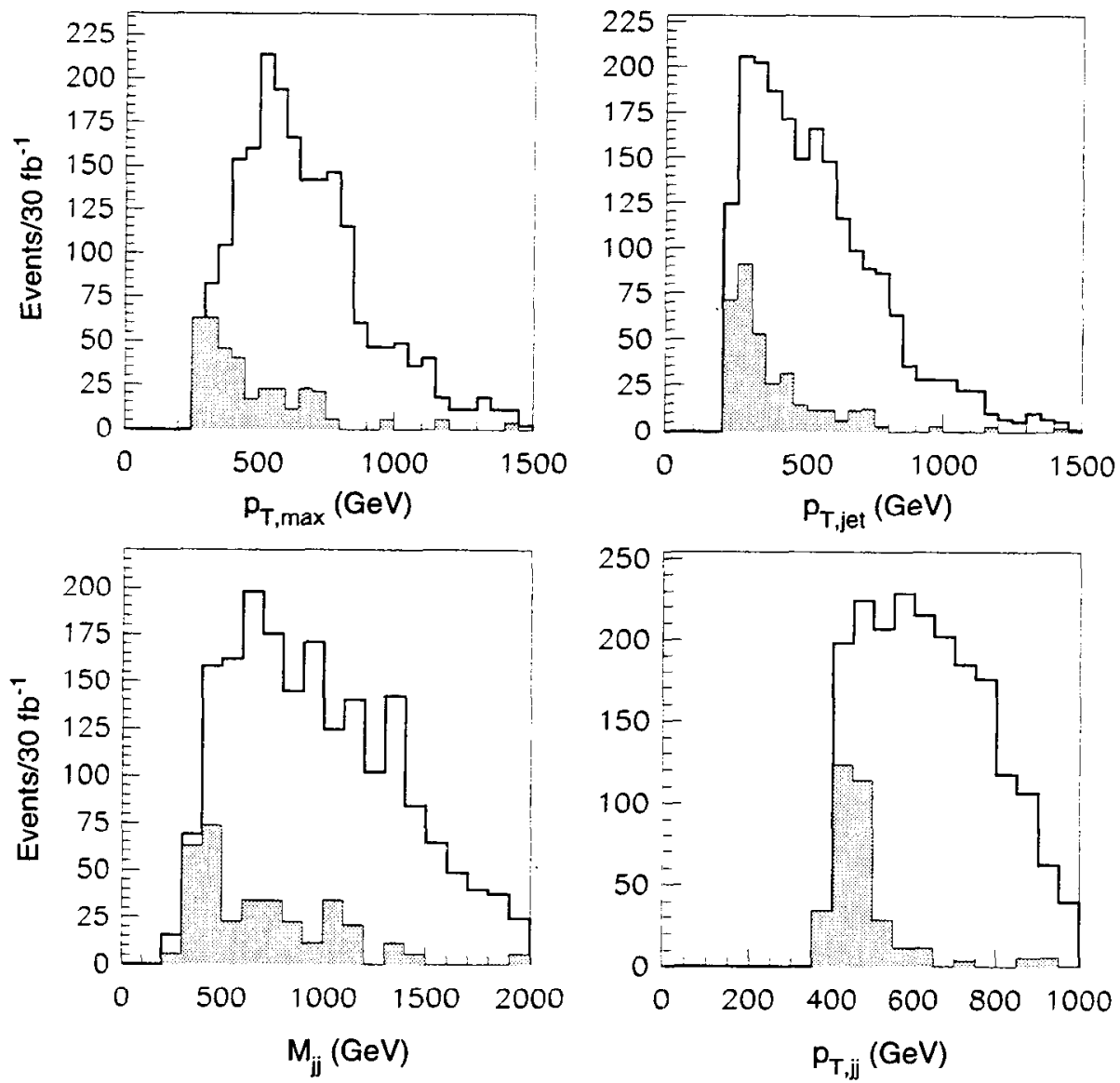

Figure 20-35 Distributions for the signal plus background (curves) and background (shaded) of the $p_{\mathrm{T}}$ of the hardest jet and the hardest two jets, the dijet mass, and the dijet $p_{\mathrm{T}}$ for the $\tilde{q}_{R}$ production signal at Point 1.

- no additional jets with $|\eta|<5$ and $p_{\mathrm{T}}>15 \mathrm{GeV}$ at low luminosity or $p_{\mathrm{T}}>40 \mathrm{GeV}$ at high luminosity;

- no leptons or $\tau$-jets.

After these cuts the SUSY signal is dominated by $\tilde{q}_{R} \tilde{q}_{R}$ pairs and $\tilde{q}_{R}$ associated production, and the Standard Model background is quite small. The resulting $p_{T}$ and mass distributions for the two hard jets are shown in Figure 20-35. The relative backgrounds for high luminosity are slightly worse because of the need to relax the jet veto. While these distributions do not exhibit any endpoints or other structure that can be directly related to masses, they are still sensitive to the squark mass. Figure 20-36 shows the $p_{\mathrm{T}}$ distributions for three different squark masses; a Kolmogorov test using these shapes is able to distinguish masses differing by about $\pm 25 \mathrm{GeV}$. After systematic effects - including the jet and $E_{\mathrm{T}}^{\text {miss }}$ energy scales, uncertainty in QCD effects such as initial state radiation, and the error in the $\tilde{\chi}_{1}^{0}$ mass - are taken into account, the sensitivity of this distribution to the $\tilde{q}_{R}$ mass is estimated to be about $\pm 40 \mathrm{GeV}$ for $30 \mathrm{fb}^{-1}$ and about $\pm 15 \mathrm{GeV}$ for $300 \mathrm{fb}^{-1}$ [20-23]. 


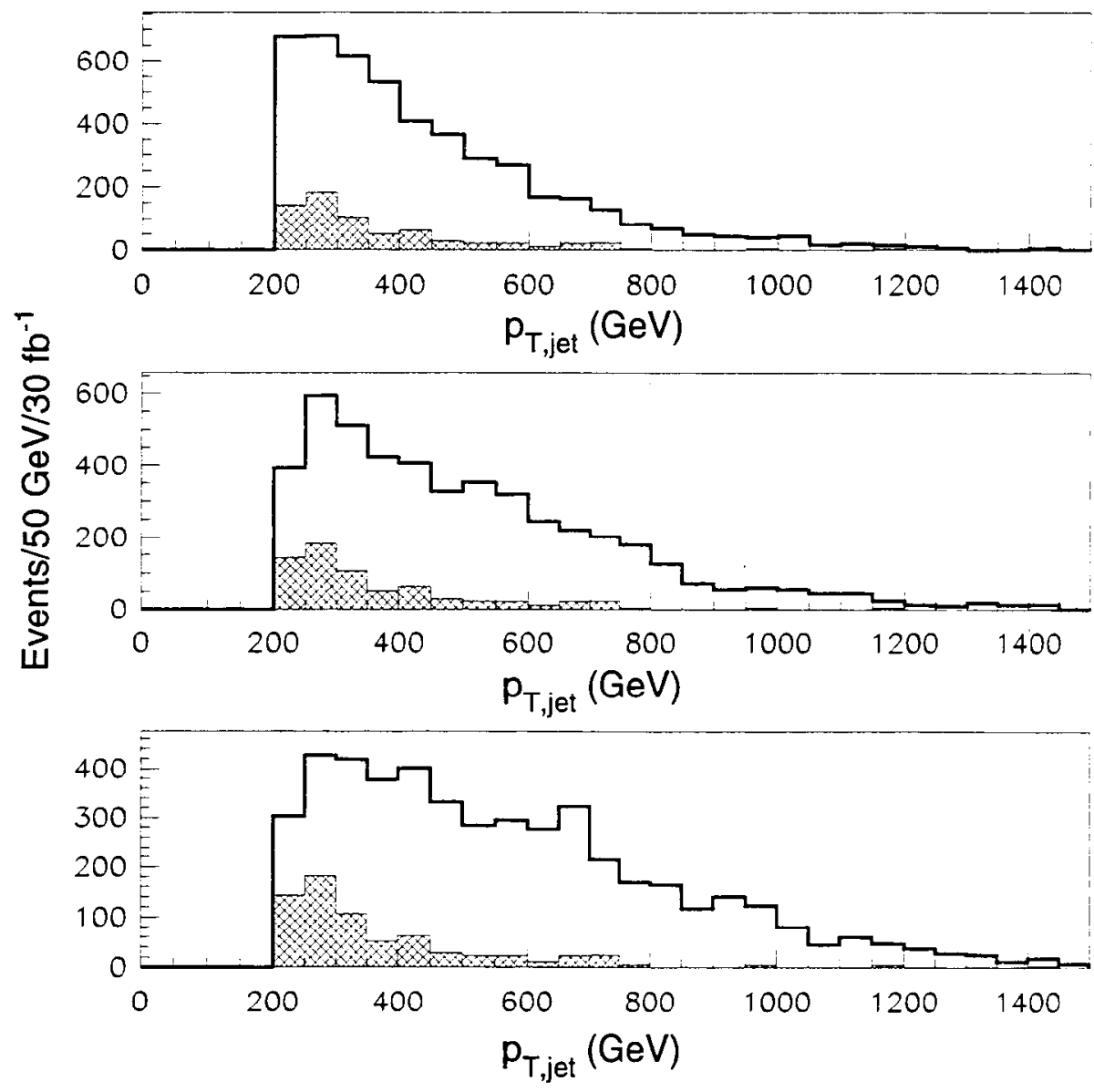

Figure 20-36 Jet $p_{\mathrm{T}}$ distributions for three different squark masses, 750,950 , and $1150 \mathrm{GeV}$, at Point 1 (full lines), and the sum of all backgrounds (hatched).

\subsubsection{2 $\tilde{\chi}_{2}^{ \pm}$signature at Point 4}

Point 4 was chosen to be close to the boundary for radiative electroweak symmetry breaking. Hence, $\mu$ is relatively small, and there is a large mixing between gauginos and Higgsinos. Production of the heavier charginos and neutralinos is therefore enhanced; about $85 \%$ of the $Z \rightarrow l^{+} l^{-}$peak in Figure 20-10 comes from $\bar{\chi}_{2}^{ \pm} \rightarrow \bar{\chi}_{1}^{ \pm} Z$.

To select these events and reject Standard Model backgrounds the following rather tight requirements were imposed [20-25]:

- two isolated, opposite-sign, same-flavour leptons with $p_{\mathrm{T}}>20 \mathrm{GeV}$ and $|\eta|<2.5$ having $87<M_{l l}<97 \mathrm{GeV}$;

- a third isolated lepton with $p_{T}>10 \mathrm{GeV}$ and $|\eta|<2.5$;

- $E_{\mathrm{T}}^{\text {miss }}>100 \mathrm{GeV}$;

- at least four jets with $p_{\mathrm{T}}>100,50,40,40 \mathrm{GeV}$ and $|\eta|<3.2$.

The last three cuts are mainly intended to remove the Standard Model backgrounds. After these cuts there about 250 signal events, 60 background events from other SUSY processes, and $4 t \bar{t}$ events, the dominant Standard Model background, for an integrated luminosity of $30 \mathrm{fb}^{-1}$. 
The rate for $Z \rightarrow l^{+} l^{-}$in SUSY events provides a rough estimate of the $\tilde{\chi}_{2}^{ \pm}$mass, $240<M\left(\bar{\chi}_{2}^{+}\right)<400 \mathrm{GeV}$. The mean transverse momentum $\left\langle p_{\mathrm{T}}^{Z}\right\rangle$ of the $Z$ is also sensitive to the masses [20-25]. A full analysis would require comparing all the relevant distributions with many samples of events varying all the model parameters. As a first step, several SUSY samples were generated changing $M\left(\tilde{\chi}_{2}^{ \pm}\right)$keeping everything else constant [2025]. The $\left\langle p_{T}^{Z}\right\rangle$ for these samples is shown in Figure 20-37. The mean can be measured to an accuracy of $\pm 7 \mathrm{GeV}$ for $10 \mathrm{fb}^{-1}$ and $\pm 3 \mathrm{GeV}$ for $100 \mathrm{fb}^{-1}$; the corresponding statistical errors on the mass are three times greater. The systematic errors are unimportant compared to the statistical errors. Various systematic effects on the relation between $\left\langle p_{\Upsilon}^{Z}\right\rangle$ and $M\left(\bar{\chi}_{2}^{ \pm}\right)$ could be important and were considered [2025]. If initial state radiation is switched off completely, $\left\langle p_{\mathrm{T}}^{Z}\right\rangle$ decreases by only $5 \mathrm{GeV}$. The

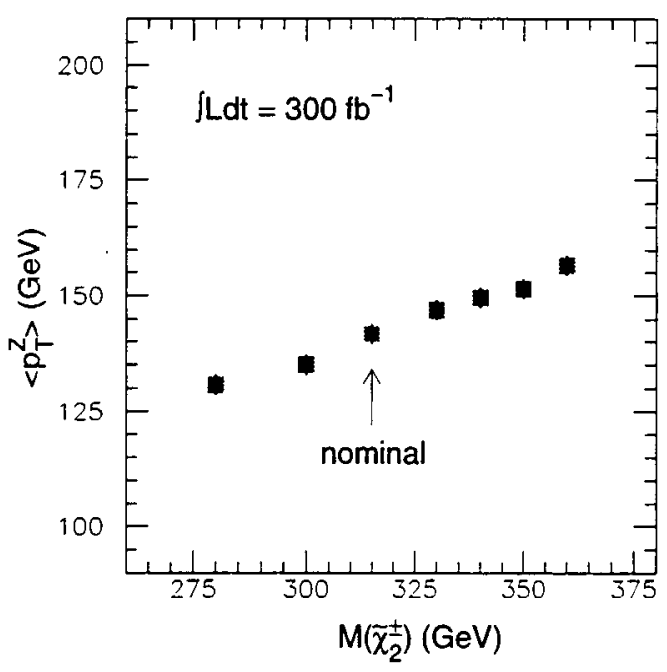
corresponding error on the $\chi_{2}^{\frac{t}{2}}$ mass should be much smaller than this. The value of $\left\langle p_{T}^{Z}\right\rangle$ also depends on the gluino and $\chi_{1}^{ \pm}$masses. Within the minimal SUGRA framework, these have estimated errors from other measurements of $\pm 10 \mathrm{GeV}$ and $\pm 2.5 \mathrm{GeV}$ respectively, and variations within these limits have a negligible effect on the $\chi_{\frac{1}{2}}^{ \pm}$mass measurement. The final estimate of the result from this method is therefore $M\left(\tilde{\chi}_{2}^{\frac{ \pm}{2}}\right)=315 \pm 21 \pm 9 \mathrm{GeV}$ for low luminosity and $M\left(\tilde{\chi}_{2}^{\frac{t}{2}}\right)=315 \pm 7 \pm 4 \mathrm{GeV}$ for high luminosity, where the first error is statistical and the second is systematic.

\subsubsection{Top production signature at Point 5}

At Point 5 there is significant top production in SUSY events from $\tilde{t}_{1} \rightarrow \tilde{\chi}_{i}^{0} t$ and $\tilde{b}_{1} \rightarrow \tilde{\chi}_{1} t$, where the $\tilde{t}_{1}$ and $\tilde{b}_{1}$ may be produced either directly or from gluino decays. Since there are not enough constraints to reconstruct a missing neutrino, it is necessary to rely on hadronic top decays. The following cuts were used to make a first selection of events [20-26]:

- $E_{\mathrm{T}}^{\text {miss }}>200 \mathrm{GeV}$;

- no leptons and $\tau$-jets;

- two and only two tagged $b$-jets with $p_{\mathrm{T}}>30 \mathrm{GeV}$;

- at least four additional central jets with $p_{\mathrm{T}}>30 \mathrm{GeV}$ and $|\eta|<3.0$.

Top candidates were then selected from this sample of events:

1. Pairs $j j$ of jets not tagged as $b$ 's with a mass $M_{j j}=M_{W} \pm 15 \mathrm{GeV}$ were selected, and the mass $M_{j j b}$ of these with one of the tagged $b$-jets was calculated.

2. The pairings were required to be unique, so no jet is used twice.

3. The pairing that minimised $\chi^{2}=\left(M_{j j b}^{(1)}-M_{t}\right)^{2}+\left(M_{j j b}^{(2)}-M_{t}\right)^{2}$ was chosen.

4. The $j j$ momenta were rescaled to obtain the correct $W$ mass, and the $j j b$ mass was recomputed. 


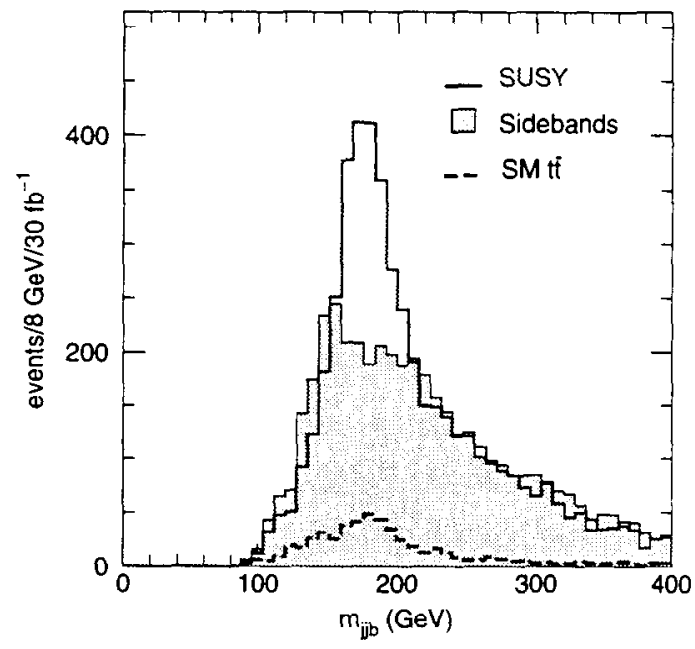

Figure 20-38 Top signal (open), estimated combinatorial background (shaded) at Point 5, and SM $t i$ production.

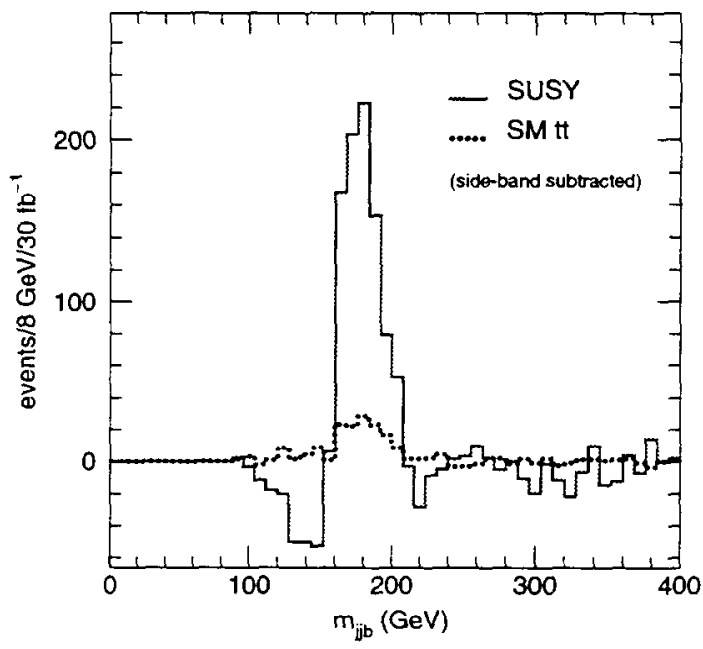

Figure 20-39 Background-subtracted top signal at Point 5.

Each step narrows the width of the $M_{j j b}$ peak at the top mass. The final result after all four steps is shown in Figure 20-38, where the SUSY combinatorial background was estimated using the $W$ sidebands $M_{j j}=50 \pm 15 \mathrm{GeV}$ and $M_{j j}=110 \pm 15 \mathrm{GeV}$. The peak with the combinatorial background subtracted is shown in Figure 20-39. For an integrated luminosity of $30 \mathrm{fb}^{-1}, 1200$ SUSY events are expected having a $t i$ pair with reconstructed masses within $\pm 30 \mathrm{GeV}$ of the top mass. (It should be noted, however, that this analysis is based on PYTHIA 2.08; ISAJET 7.27 predicts a smaller signal). Since direct $\tilde{t}_{1}$ production is small, the observation of $t \bar{t}$ provides an indication that $\tilde{g} \rightarrow \tilde{t}_{1} \dot{t} \rightarrow \tilde{\chi}_{1}^{0} t \bar{i}$ is kinematically allowed. Partial reconstruction of either the direct production or the gluino decay is not easy due to the large SUSY backgrounds but could provide additional constraints on the SUSY model [20-26].

\subsubsection{Direct slepton production at Point 5}

At Point 5 , while the $\tilde{l}_{R}$ are copiously produced in cascade decays involving the $\tilde{\chi}_{2}^{0}$, the $\tilde{l}_{L}$ are produced mainly through the Drell-Yan process $q \bar{q} \rightarrow \gamma^{*}, Z^{*} \rightarrow \bar{l}_{L}^{+} \tilde{l}_{\bar{L}}$. Since the branching ratio for $\tilde{l}_{L} \rightarrow \tilde{\chi}_{1}^{0} l$ is larger than $95 \%$, the signature is an acoplanar pair of opposite-sign, same-flavour leptons, large $E_{\mathrm{T}}^{\text {miss }}$, and no additional jets. The potential backgrounds from $t \dot{t}$ and from other SUSY processes are much larger than the signal, so rather hard cuts are necessary.

The signal and the Standard Model and SUSY backgrounds were generated with PYTHIA, and the detector response was simulated with ATLFAST. For low luminosity the following cuts were applied [20-26]:

- $E_{\mathrm{T}}^{\text {miss }}>120 \mathrm{GeV}$;

- two opposite-sign, same-flavour leptons (lepton $\equiv e, \mu$ ) with $p_{\mathrm{T}}>30 \mathrm{GeV}$ and $|\eta|<2.5$;

- angular separation $\Delta \phi<2$ for the two leptons;

- no jets with $p_{\mathrm{T}}>15 \mathrm{GeV}$ and $|\eta|<5$. 
Such a strict jet veto is needed to reduce the $t \dot{t}$ background to an acceptable level, while the $\Delta \phi$ cut helps to reduce the $W W$ background. These cuts also essentially eliminate the $\tilde{l}_{R} \tilde{l}_{R}$ background, which gives leptons that are softer and/or more collinear. The jet veto is sufficient to make the other SUSY backgrounds negligible. After these cuts, there is a total of 58 signal events, with backgrounds of $14 \mathrm{WW}$ and four $t \dot{t}$ events for an integrated luminosity of $30 \mathrm{fb}^{-1}$.

Since the number of events is small, it is important to extend this search to high luminosity. This requires relaxing the jet veto. The last two cuts above were replaced by:

- angular separation $\Delta \phi<2.5$ for the two leptons;

- no jets with $p_{\mathrm{T}}>40 \mathrm{GeV}$ and $|\eta|<5$.

With these cuts there are a total of 600 signal events with backgrounds of $140 \mathrm{WW}$ events and $140 t i$ events for an integrated luminosity of $300 \mathrm{fb}^{-1}$. While the signatures for sleptons are obviously difficult, it would be possible to detect the $\tilde{l}_{L}$ at this point with ATLAS using the full luminosity of the LHC.

The dilepton signal does not provide a direct measurement of the slepton mass. Since the Drell-Yan cross section can be calculated with reasonable accuracy, it is possible to estimate
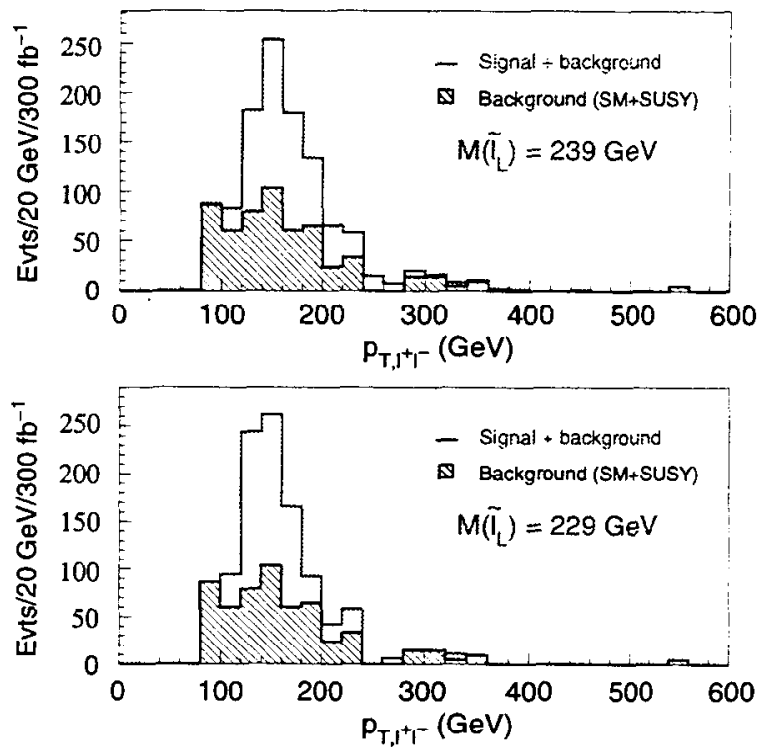

Figure 20-40 Distribution of the $p_{\mathrm{T}}$ of the selected dilepton pairs for two values of the $l_{R}$ mass. the mass using the observed rate, but the accuracy will be limited by the knowledge of the efficiency of the cuts and by the uncertainty on the branching ratio and on the luminosity measurement (See Chapter 13). There is also sensitivity to the mass in the kinematic distributions, specifically in the $p_{T}$ of the dilepton pair. This distribution is shown in Figure 20-40 for two different values of the slepton mass. A very precise measurement of the mass seems difficult.

If the $\tilde{l}_{L}$ were slightly heavier, then it would also be possible to search for a four-lepton signal from one $\tilde{l}_{L} \rightarrow \tilde{\chi}_{1}^{0} l$ decay and one $\tilde{l}_{L} \rightarrow \tilde{\chi}_{2}^{0} l \rightarrow \tilde{\chi}_{1}^{0} l l l$ decay. In principle the endpoint of the trilepton mass can be used to determine the slepton mass, although the rates are marginal. Such a case is discussed in Section 20.2 .10 below.

\subsubsection{Exclusive SUGRA measurements for large $\tan \beta$}

SUGRA phenomenology for $\tan \beta \geq 10$ is more complicated because of the need to include additional Yukawa couplings and mixings in the $\tilde{b}$ and $\tilde{\tau}$ sectors. For this technical reason the five LHCC SUGRA points were all selected to have $\tan \beta \leq 10$. Hence, at least one of the modes $\tilde{\chi}_{2}^{0} \rightarrow \tilde{\chi}_{1}^{0} l l, \tilde{\chi}_{2}^{0} \rightarrow \tilde{l} l$, or $\tilde{\chi}_{2}^{0} \rightarrow \tilde{\chi}_{1}^{0} h$ was available for each point to provide a good starting point for exclusive measurements. There is, however, no reason to disfavour larger values of $\tan \beta$. The most important effect of large $\tan \beta$ is that the splitting between the $\tilde{\tau}_{1}$ and the other sleptons is increased. If $m_{1 / 2}$ is small enough so that $\tilde{\chi}_{2}^{0} \rightarrow \bar{\chi}_{1}^{0} h$ and $\tilde{\chi}_{2}^{0} \rightarrow \tilde{\chi}_{1}^{0} Z$ are forbidden and $m_{0}$ large enough that $\chi_{2}^{0} \rightarrow \tilde{l}_{L, R} l$ is also forbidden, then the only allowed two-body decays for the light gauginos can be $\tilde{\chi}_{2}^{0} \rightarrow \tilde{\tau} \tau$ and $\chi_{1}^{ \pm} \rightarrow \tilde{\tau} v_{\tau}$. These modes are then dominant, and none of the signatures discussed in Section 20.2.3 - 20.2.7 can be used. 
SUGRA Point 6 in Table 20-3 is an example of such a point. The masses are listed in Table 20-4. Since the masses are light to avoid $\tilde{\chi}_{2}^{0} \rightarrow \tilde{\chi}_{1}^{0} h$, the cross section is large, $99 \mathrm{pb}$. Some of the most important branching ratios are

$$
\begin{array}{rlrl}
B R\left(\tilde{\chi}_{2}^{0} \rightarrow \tilde{\tau}_{1} \tau\right) & =99.9 \% & B R\left(\tilde{\chi}_{1}^{ \pm} \rightarrow \tilde{\tau}_{1} \nu_{\tau}\right) & =99.9 \% \\
B R\left(\tilde{\chi}_{3}^{0} \rightarrow \tilde{\chi}_{2}^{0} Z\right) & =13 \% & B R\left(\tilde{\chi}_{3}^{0} \rightarrow \tilde{\tau}_{1} \tau\right) & =21 \% \\
B R\left(\tilde{g} \rightarrow \tilde{b}_{1} b\right) & =55 \% & B R\left(\tilde{g} \rightarrow \tilde{b}_{2} b\right) & =10 \% \\
B R\left(\tilde{g} \rightarrow \tilde{q}_{L} q\right) & =3 \% & B R\left(\tilde{g} \rightarrow \tilde{q}_{R} q\right) & =5.7 \%
\end{array}
$$

To study this point it is essential to use $\tau$ decays. Since SUSY events have additional missing energy from the $\tilde{\chi}_{1}^{0}$, it is not possible to reconstruct the $\tau$ momenta using the missing transverse energy as is done for $A \rightarrow \tau \tau$ in Section 9.3.3.4. Therefore, this analysis relies mainly on hadronic $\tau$ decays; the selection is biased towards multi-body decays, e.g., $\tau \rightarrow \rho v$ or $\tau \rightarrow a_{1} v$, so that the visible momentum is closer to the true $\tau$ momentum.

ISAJET 7.37 was used [20-33] to generate a total of 600000 signal events. Because the masses are low, the cross section is large; this sample corresponds to an integrated luminosity of about $6 \mathrm{fb}^{-1}$. The response of ATLAS to these events was simulated with a simple particle-level simulation, including Gaussian resolutions and overall coverage but no cracks or non-Gaussian tails. Jets with $p_{\mathrm{T}}>25 \mathrm{GeV}$ were found using a simple fixed-cone jet algorithm with $R=0.4$. Leptons were required to have $p_{\mathrm{T}}>10 \mathrm{GeV}$ and $|\eta|<2.5$ and to be isolated, with $E_{\mathrm{T}}<10 \mathrm{GeV}$ in a $R=0.2$ cone. An additional efficiency of $90 \%$ is included for each lepton. Where relevant, jets were assumed to be misidentified as photons with a probability of $10^{-3}$, significantly worse than what is actually expected.

Such a simple, particle-level simulation is inadequate to describe the measurement of $\tau$ 's; for example, the conversion of photons in the tracker has a non-trivial effect on the observed multiplicity in jets. Hence, a parametrisation of the $\tau$ response based on full GEANT simulation was developed [20-34]. Several samples of $Z \rightarrow \tau \tau$ events in various $p_{\mathrm{T}}$ ranges were generated with PYTHIA, and the ATLAS response was simulated with DICE and ATRECON. An algorithm to identify $\tau$-jets based on the jet mass, multiplicity, and isolation criteria based on this simulation was then developed. The algorithm, described in Section 9.3.3.2, was optimised for $\tau \tau$ mass resolution rather than $\tau$ identification, so it is biased both against single pion decays and against jet masses greater than $M_{\tau}$. It accepts $41 \%$ of the $\tau$ hadronic decays while giving a rejection of about 15 against light quark jets, which is adequate for the purpose. The mean value of the visible mass is $M_{\text {vis }}=0.66 M_{\tau \tau}$ for all of the $p_{\mathrm{T}}$ ranges considered, and the resolution is approximately Gaussian with $\sigma / M_{\text {vis }}=0.12$. The sign of the $\tau$ is identified correctly $92 \%$ of the time, from the charge of the tracks ascribed to the $\tau$ decay.

These results from full simulation provided the basis for $\tau$ identification and reconstruction in the SUSY and the Standard Model background samples. The jets found from the particle-level simulation were compared with the $\tau$ 's from the event generator. If there was a $\tau$ within $\Delta R<0.4$ of a jet and its hadronic decay products satisfied $E_{\tau}>0.8 E_{\text {jet }}$, the jet was assumed to be a $\tau$; otherwise it was assumed to be a normal jet. The $\tau$ identification or misidentification probabilities from the full simulation were then applied to obtain the reconstructed $\tau$ 's. Masses for $\tau \tau$ pairs were calculated by smearing the generated masses with the resolution and shift from the full simulation rather than by using the jets. The charges were also assigned using the probabilities from the full simulation. This approach makes it possible to apply at least the main results from the GEANT simulation to large event samples. 


\subsubsection{Effective mass}

The first step in the analysis is to look for a deviation from the Standard Model with a strategy similar to that for moderate $\tan \beta$. Gluinos and squarks decay to give signatures with multiple jets (including $\tau$-jets) and missing energy. Events were selected with cuts very similar to those used in Section 20.2.1:

- at least four jets, some of which may be $\tau$ 's, with $p_{\mathrm{T}}>100,50,50,50 \mathrm{GeV}$;

- $E_{\mathrm{T}}^{\text {miss }}>\max \left(100 \mathrm{GeV}, 0.2 \mathrm{M}_{\mathrm{eff}}\right)$;

- transverse sphericity $S_{\mathrm{T}}>0.2$;

- no $\mu$ or isolated $e$ with $p_{\mathrm{T}}>20 \mathrm{GeV}$ and $|\eta|<2.5$.

Figure 20-41 shows the distribution of effective mass $M_{\text {eff }}$ after these cuts. As before, the signal dominates over the background for large $M_{\text {eff }}$ by about a factor of ten. Since the

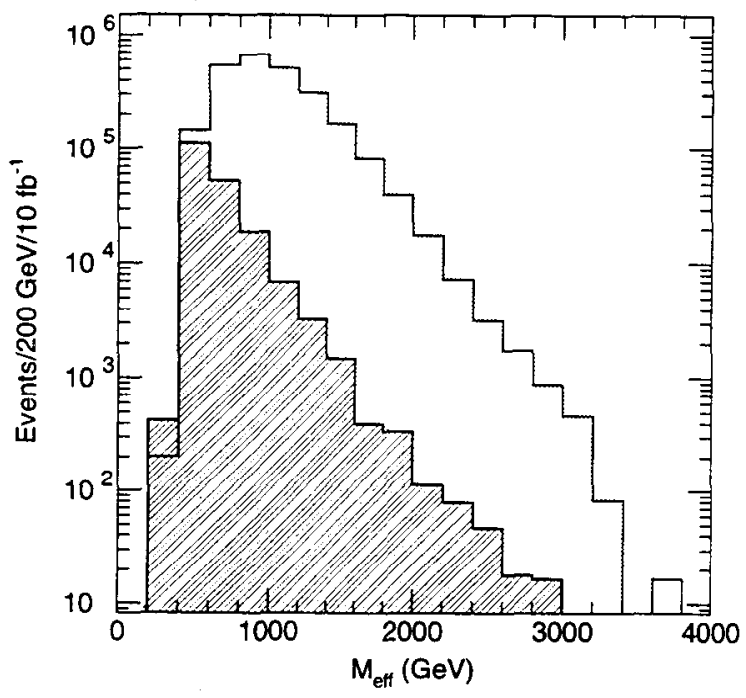

Figure 20-41 Effective mass distribution for Point 6 signal (open) and sum of Standard Model backgrounds (shaded) after cuts. masses must be fairly low to exclude $\tilde{\chi}_{2}^{0} \rightarrow \tilde{\chi}_{1}^{0} h$, the cross section is large. Discovery of this signal is therefore easy and does not depend on measuring $\tau$ 's.

\subsubsection{2 $\tau \tau$ mass distribution}

It can be seen from Figure 20-41 that the SUSY signal dominates over the Standard Model background if the lepton cut is replaced by: $M_{\text {eff }}>500 \mathrm{GeV}$.

Two $\tau$ 's are also required, and the lepton veto is then omitted. If the $\tau \tau$ mass distribution could be measured directly, it would show a sharp edge from $\tilde{\chi}_{2}^{0} \rightarrow \tilde{\tau} \tau$ at

$$
M_{\tau \tau}^{\max }=M_{\tilde{\chi} \varrho} \sqrt{1-\frac{M_{\tau_{1}}^{2}}{M_{\tilde{\chi}_{2}^{0}}^{2}}} \sqrt{1-\frac{M_{\tilde{\chi}_{1}^{0}}^{2}}{M_{\tilde{\tau}_{1}}^{2}}}=59.64 \mathrm{GeV}
$$

The selection discussed earlier is biased towards higher mass $\tau$ decays, for which the visible energy is closer to the true energy. The visible $\tau \tau$ mass distribution is shown in Figure 20-42 for real $\tau \tau$ pairs and for pairs made with a fake $\tau$. The edge structure is clearly visible in the real distribution, although it is of course smeared out by the missing neutrinos. The fake mass distribution is concentrated at higher masses, reflecting the larger gluino and squark masses.

Figure 20-43 shows the distribution of visible masses resulting from scaling the generated masses by $\pm 7.5 \%$ from the nominal values in order to test the sensitivity. These curves could be distinguished statistically, but understanding the background under the edge might be a problem since it is still quite large. 


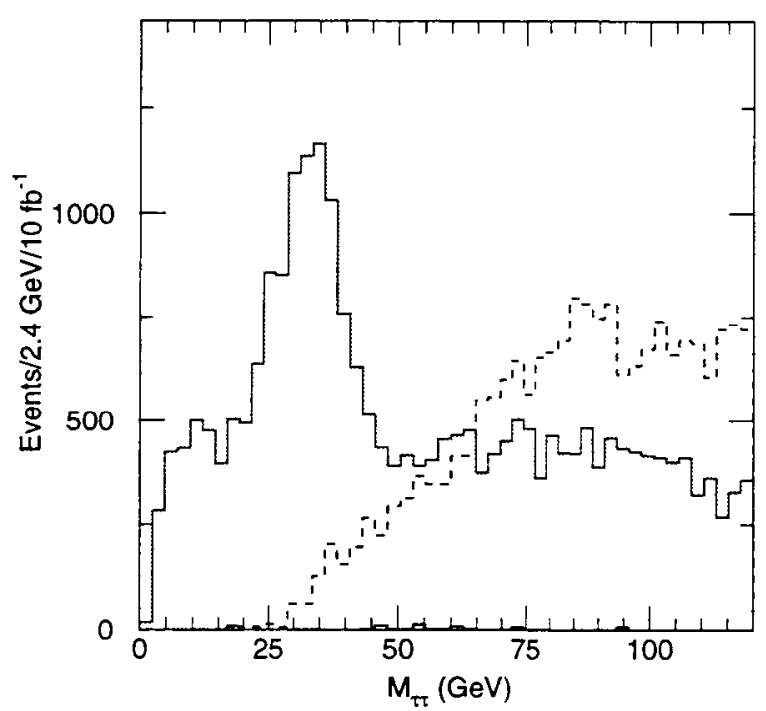

Figure 20-42 Visible $\tau \tau$ mass at Point 6 after cuts. The dashed curve is the background from fake $\tau$ 's; the shaded histogram is the Standard Model background.

Since the SUSY cross section is dominated by $\tilde{g} \tilde{g}$ and $\tilde{g} \tilde{q}$ production and the gluino is a Majorana fermion, most of the background from two $\chi_{1}^{ \pm} \rightarrow \tilde{\tau}^{ \pm} v$ decays cancels in the $\tau^{+} \tau^{-}-\tau^{ \pm} \tau^{ \pm}$combination. The fake $\tau$ background has random signs, so it also cancels. The $\tau^{+} \tau^{-}-\tau^{ \pm} \tau^{ \pm}$mass distribution is shown in Figure 20-44. The fake background is much smaller and the signal to background ratio for the edge is much better than in the previous figures. The signal beyond the endpoint is real and comes from $\tilde{\chi}_{3}^{0}$ and $\tilde{\chi}_{4}^{0}$ decays. Since the real fluctuations would be smaller than those in Figure 20-43, and $\tau$ decays are well understood, an error of $5 \%$ on the $\tau \tau$ endpoint should be achievable. The dominant error probably comes from the uncertainty on the effect of the $\tau$ identification cuts and so is difficult to estimate without much more study.

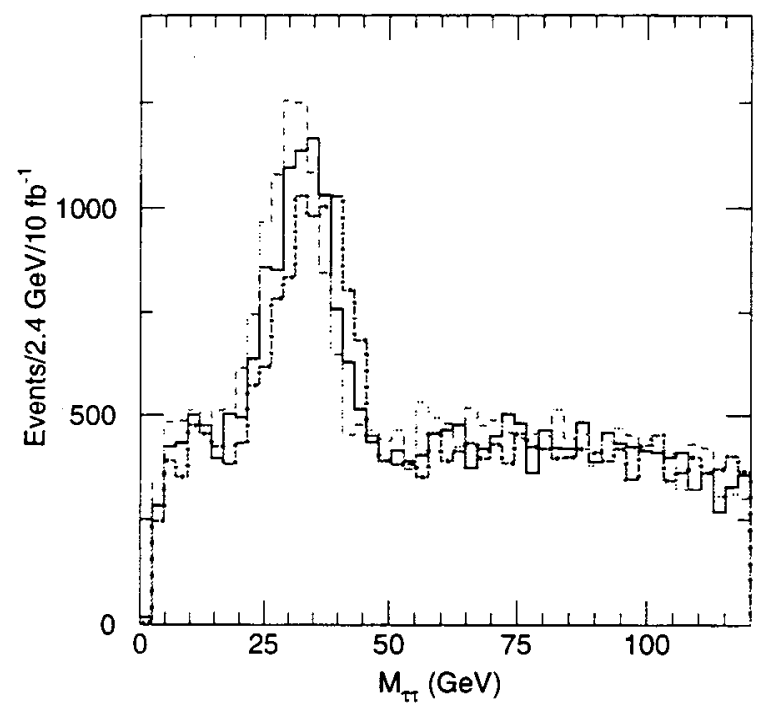

Figure 20-43 Visible mass distribution for real $\tau \tau$ pairs from Figure 20-42 compared with same distributions scaled by $\pm 7.5 \%$.

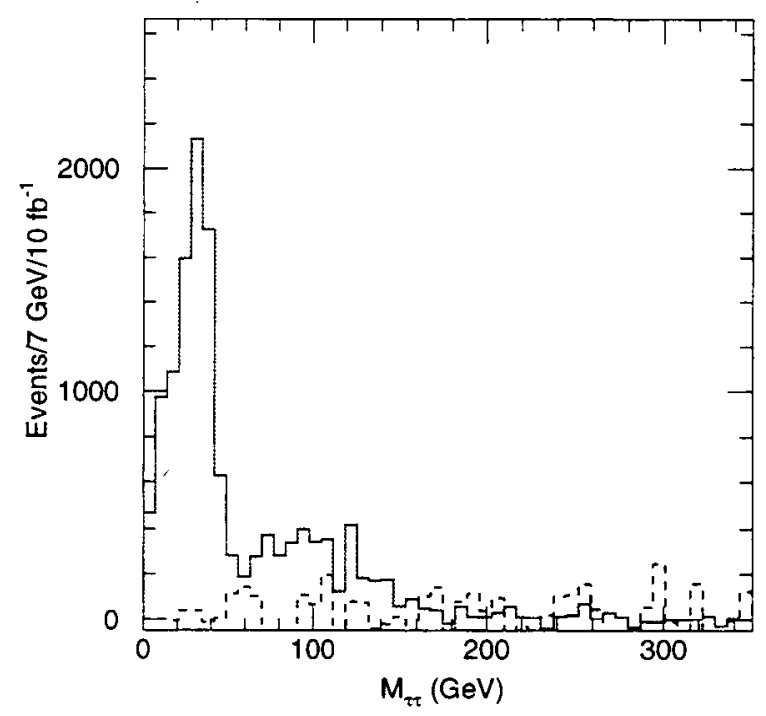

Figure 20-44 $\tau^{+} \tau^{-}-\tau^{ \pm} \tau^{ \pm}$mass distribution for Point 6 signal (solid) and for background from fake $\tau$ 's (dashed).

\subsubsection{Reconstruction of $\tilde{g} \rightarrow \tilde{b} b \rightarrow \tilde{\chi}_{1}^{0} \tau \tau b b$}

At Point 5 it was possible to combine a $l^{+} l^{-}$pair with a hard jet to obtain a signal for $\tilde{q}_{L}$ (see Section 20.2.4.3). While a similar analysis is possible in principle for Point 6 using a $\tau^{+} \tau^{-}$pair, it is much more difficult. It seems necessary both to use heavy flavour tagging and to reconstruct the gluino to get a good signal. 


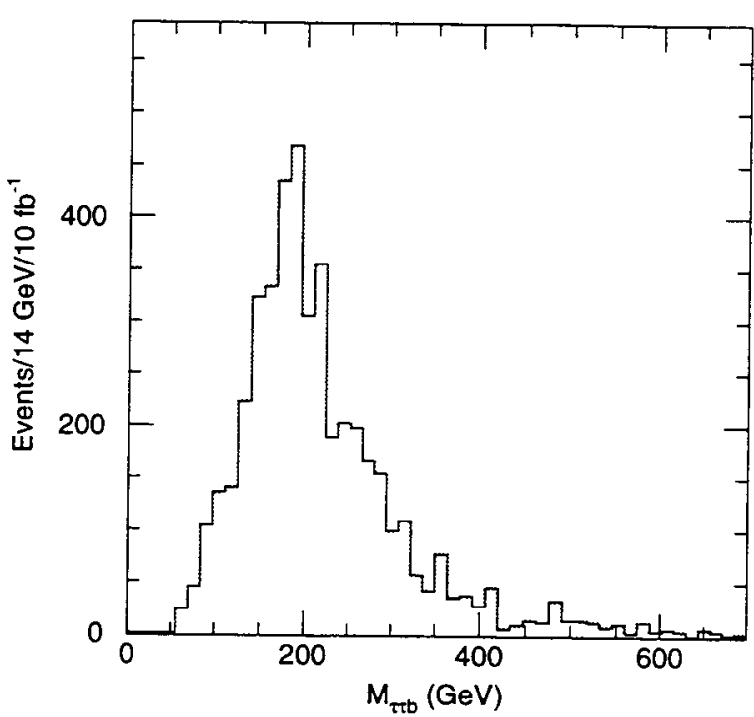

Figure 20-45 Visible $\tau^{+} \tau^{-}+$jet mass at Point 6 for tagged $b$-jets. The distribution for like-sign $\tau$ pairs has been subtracted.

The same jet, effective mass, and missing energy cuts were made as before. Events were then selected to have:

- two $\tau$ 's with a visible $M_{\tau \tau}$ within $10 \mathrm{GeV}$ of the peak in Figure 20-42;

- at least one tagged $b$-jet with $p_{\mathrm{T}}>25 \mathrm{GeV}$.

The resulting $\tau^{+} \tau^{-} b-\tau^{ \pm} \tau^{ \pm} b$ visible mass distribution is shown in Figure 20-45. If the $\tau$ 's were perfectly measured, this would have an endpoint at

$$
\begin{aligned}
M_{\tau \tau b}^{\max } & =\left[\frac{\left(M_{\tilde{b}_{1}}^{2}-M_{\bar{\chi}_{2}^{0}}^{2}\right)\left(M_{\bar{\chi}_{2}^{0}}^{2}-M_{\tilde{\chi}_{1}^{0}}^{2}\right)}{M_{\tilde{\chi}_{2}^{0}}^{2}}\right]^{1 / 2} \\
& =303.9 \mathrm{GeV}
\end{aligned}
$$

analogous to that for Point 5 in Figure 20-20. There is perhaps some structure but nothing like a real endpoint. The distribution is, however, clearly different from the same distribution with non- $b$ jets shown in Figure 20-46.

A somewhat better signal can be obtained by requiring two tagged $b$-jets and reconstructing the gluino and squark simultaneously. This is similar to what was done for Point 3 in Section 20.2.4.1. Events were selected with a visible mass $40<M_{\tau \tau}<60 \mathrm{GeV}$, and the momentum of the $\tilde{\chi}_{2}^{0}$ was estimated using the analog of Equation 20-7. This approximation is not as good as in the previous case: because this is a two-step decay, the $\tilde{\chi}_{1}^{0}$ momentum would not vanish at the endpoint even if the $\tau$ 's were perfectly measured. Also, the difference $M_{\tilde{g}}-M_{\tilde{b}}$ is larger in this case, so the resolution on it is not as good. Nevertheless, the plot, Figure $20-47$, of
Figure 20-46 Visible $\tau^{+} \tau^{-}+$jet mass at Point 6 for light quark jets.

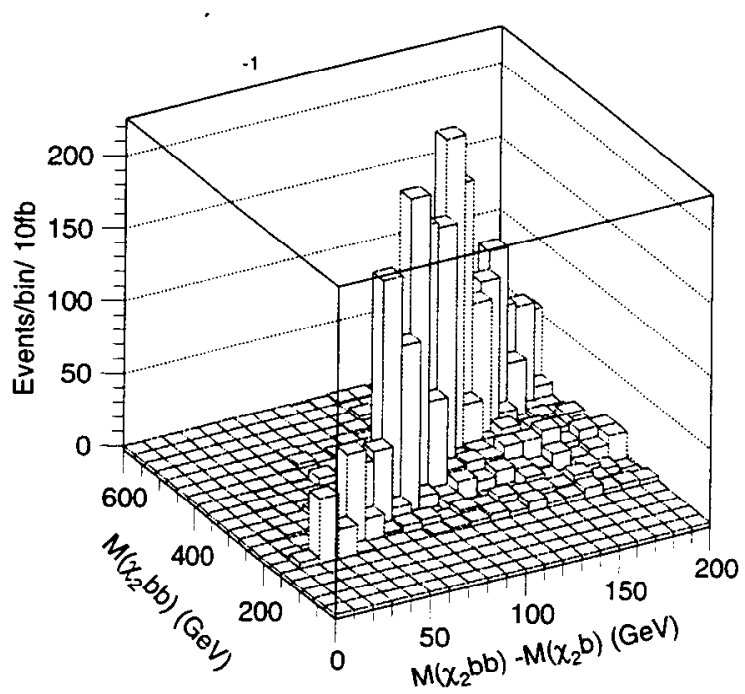

Figure 20-47 Lego plot of the reconstructed $M_{\bar{g}}$ vs $M_{\bar{g}}-M_{\tilde{b}_{1}}$ at Point 6. 


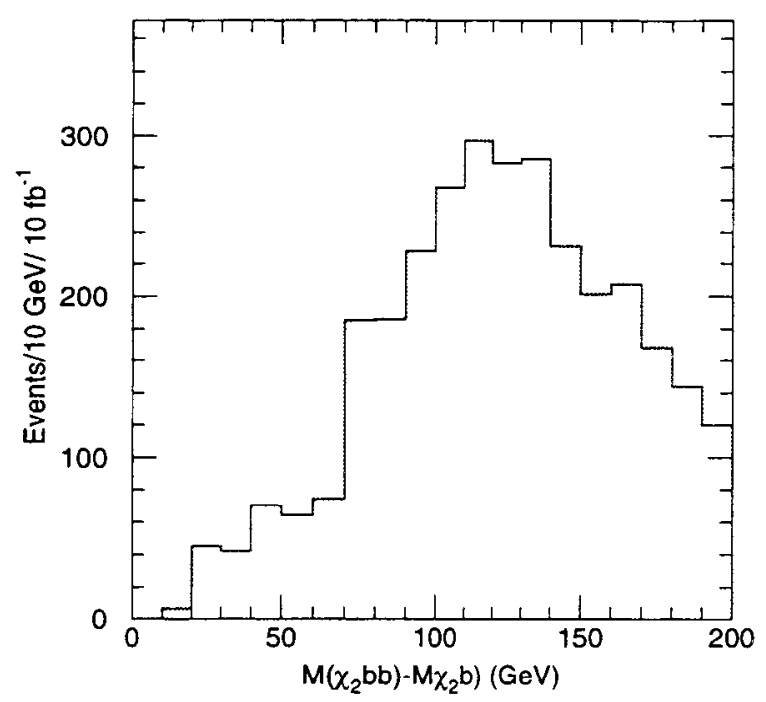

Figure 20-48 Projection of Figure 20-47 onto the $M_{\tilde{g}}-M_{\bar{b}_{1}}$ axis.

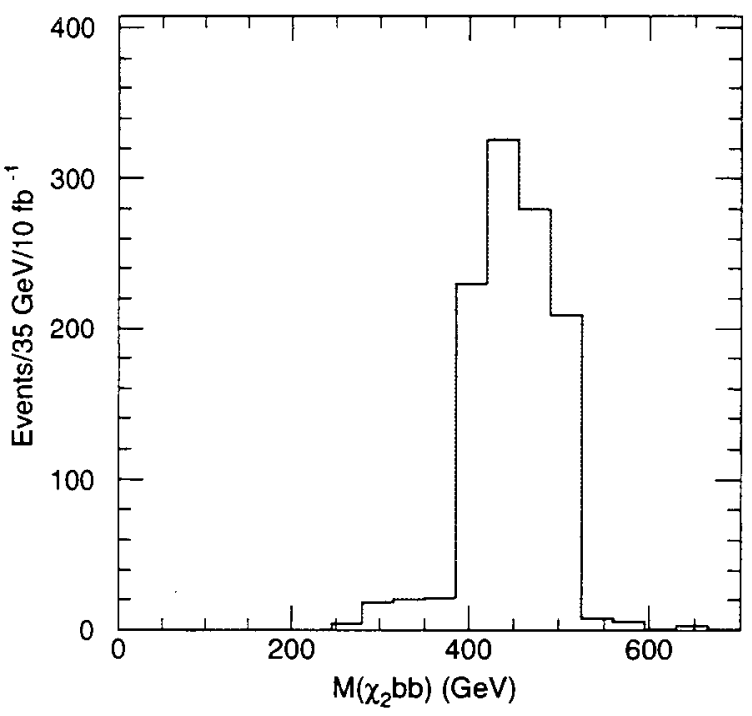

Figure 20-49 Projection of Figure 20-47 onto the $M_{\tilde{g}}$ axis with the cut $100 \mathrm{GeV}<M_{\tilde{g}}-M_{\tilde{b}_{1}}<140 \mathrm{GeV}$.

$M_{\bar{g}}-M_{\tilde{b}_{1}}$ vs $M_{\tilde{g}}$ reconstructed in this way does show a peak at the right place. The projection onto the $M_{\tilde{g}}-M_{\tilde{b}}$ axis is shown in Figure 20-48 and the second projection, after cutting on $M_{\tilde{g}}-M_{\bar{b}_{1}}$, is shown in Figure 20-49.

A detailed estimate of the errors to be expected from this sort of analysis would require generating many signal samples and comparing the resulting distributions with the corresponding masses. This has not been done, nor has the analysis been optimised. It seems plausible, however, that for an integrated luminosity of $30 \mathrm{fb}^{-1}$ the mass difference $M_{\tilde{g}}-M_{\tilde{b}_{1}}$ could be determined to about $\pm 20 \mathrm{GeV}$ while $M_{\tilde{g}}$ could be determined to $\pm 60 \mathrm{GeV}$. These error estimates are used for the fits in Section 20.2.9 below.

\subsubsection{Reconstruction of $\tilde{q}_{R} \rightarrow \tilde{\chi}_{1}^{0} q$}

The $\tau \tau$ signatures at Point 6 come primarily from $\tilde{q}_{L}$ decays: It is also possible to produce $\tilde{q}_{R} \tilde{q}_{R}$ pairs, either directly or from gluino decays. Since for squarks of the first two generations the decay $\tilde{q}_{R} \rightarrow \tilde{\chi}_{1}^{0} q$ has a branching ratio of more than $95 \%$, such events are characterised by two hard jets, no additional soft jets except those from gluino decay or initial state radiation, and large missing energy. The analysis is analogous to the one performed in Section 20.2.7.1. To select this signature, events were therefore selected to have: 
- $E_{\mathrm{T}}^{\text {miss }}>\max \left(200 \mathrm{GeV}, 0.2 M_{\text {eff }}\right)$;

- two jets with $p_{\mathrm{T}}>150 \mathrm{GeV}$;

- no additional jets with $p_{\mathrm{T}}>25 \mathrm{GeV}$;

- no leptons, $b$-jets, or $\tau$-jets.

The $p_{\mathrm{T}}$ distribution for the two hardest jets in the events that are accepted by these cuts are shown in Figure 20-50. The mean $p_{T}$ is a function of the squark mass, or rather of a complicated function of the squark production kinematics and of the energy release $M_{\tilde{q}_{R}}\left(1-M_{\tilde{\chi}_{1}^{0}}^{2} / M_{\tilde{q}_{R}}^{2}\right)$. The statistical error on the mean is tiny, so the only significant errors are systematic. The total systematic error on $M_{\tilde{q}_{R}}$ should be $25-50 \mathrm{GeV}$ for $30 \mathrm{fb}^{-1}$ and somewhat less for $300 \mathrm{fb}^{-1}$ as the errors are better understood. These errors are used in the fits in Section 20.2 .9 below.

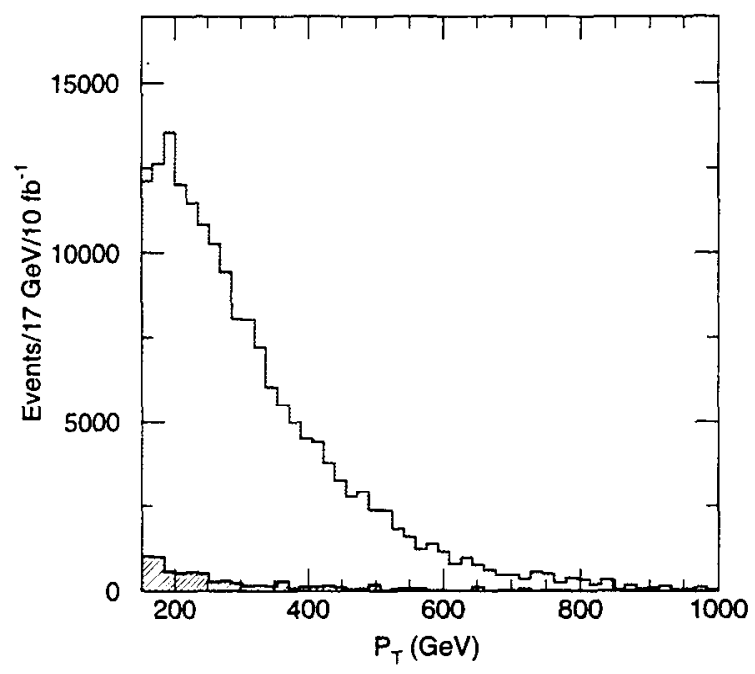

Figure 20-50 $p_{T}$ distribution of two hardest jets for Point 6 signal (solid) and Standard Model background (shaded) after cuts described in the text.

\subsubsection{Fitting minimal SUGRA parameters}

Once a number of experimental constraints on combinations of masses have been set with methods like those described above, they can be used in a global fit to determine the parameters of a SUSY-breaking model [20-22, 20-27]. Clearly this requires that the model have only a limited number of parameters. This subsection gives the results of fitting the measurements discussed above to the minimal SUGRA model. This minimal model of course can fit the data by construction. Section 20.2.10 describes how well extra parameters corresponding to a few possible extensions of the minimal model could be constrained. A general treatment of non-minimal models seems very difficult without experimental data to provide guidance.

While some model-independent masses can be determined as described in Section 20.2.6, most of the measurements correspond to combinations of masses. Fitting masses or combinations of masses is much simpler than fitting kinematic distributions: for each point in the parameter space of the model it is sufficient to calculate the spectrum rather than generating and analysing a full sample of events. Also, expressing the results in terms of masses makes it easy to combine results derived using different event generators or different versions of the same generator. Thus, for simplicity all the fits have been done using only masses or combinations of masses. For a measurement such as the $\tilde{q}_{R} \rightarrow \tilde{\chi}_{1}^{0} q$ signature described in Section 20.2.7.1, an approximate equivalent error on the squark mass was assigned. Measurements of this sort are noted below. Of course, if SUSY is discovered, a much more general analysis will be done using many event samples to fit all of the available data, also including the information from branching fractions and cross sections measurements.

The fits for Points 1-5 were made before a correct treatment of large $\tan \beta$ was available in the generator, so an artificial restriction on it had to be imposed. An initial scan of 300000 points was made covering the range 


$$
\begin{gathered}
50<m_{0}<1000 \mathrm{GeV} \\
100<m_{1 / 2}<700 \mathrm{GeV} \\
1<\tan \beta<15
\end{gathered}
$$

for both signs of $\mu$. The RGE's cause the parameters $A_{t}, A_{b}$, and $A_{\tau}$ that appear in the weakscale mass matrices to evolve to approximate fixed points with very little dependence on the GUT-scale parameter $A_{0}$, which therefore cannot be determined and was not included in the scan. For each point in the scan, all the measured masses or combinations of masses were calculated, and the point was weighted using the estimated experimental errors, artificially inflated if necessary to obtain a reasonable number of solutions. After this initial scan found an approximate allowed region, more points were generated in this smaller region until the $\pm 1 \sigma$ errors were determined for $30 \mathrm{fb}^{-1}$, corresponding to an initial three years at low luminosity, and for $300 \mathrm{fb}^{-1}$, corresponding to the ultimate reach of ATLAS. For Point 6 a similar method was used with the scan region extended to $\tan \beta<60$.

The theoretical treatment used to calculate the masses is not perfect. The RGE's are calculated to two loops in gauge couplings and one loop in Yukawa couplings, the thresholds are treated as step functions, and no GUT-scale physics is included. It is assumed that these things can be improved, so no associated errors are included in the fits. The calculation of the light Higgs mass is more difficult. The one-loop effective potential used to calculate it is rather sensitive to the choice of scale, and the leading two-loop effects from the running top quark mass are known to be important. Since the expected experimental error on the mass from $h \rightarrow \gamma \gamma$ is at the $0.1 \%$ level (see Section 19.2.2), three cases are considered here:

- Low-L: includes statistical errors for low luminosity, $30 \mathrm{fb}^{-1}$, the systematic errors on the electromagnetic, muon, and jet energy scales previously mentioned, and a theory-dominated error of $\Delta M_{h}= \pm 1 \mathrm{GeV}$ on the light Higgs mass.

- High-L: includes statistical errors for high luminosity, $300 \mathrm{fb}^{-1}$, the systematic errors on the electromagnetic, muon, and jet energy scales previously mentioned, and a theorydominated error of $\Delta M_{h}= \pm 1 \mathrm{GeV}$ on the light Higgs mass.

- Ultimate: The same as High-L but including only the experimental error $\Delta M_{h}= \pm 0.2 \mathrm{GeV}$ on the light Higgs mass.

The fits for each point will be described in turn.

Table 20-5 Inputs for the minimal SUGRA model fits at Points 1 and 2. The measurements listed below the heavy rule determine combinations of masses and production dynamics. The High-L and Ultimate measurements differ only in the error on the Higgs mass.

\begin{tabular}{llll}
\hline Quantity & Reference & Low-L & Ultimate \\
\hline$M_{h}$ (Point 1) & Section 19.2.2 & $95.4 \pm 1.0 \mathrm{GeV}$ & $95.4 \pm 0.2 \mathrm{GeV}$ \\
$M_{h}$ (Point 2) & Section 19.2.2 & $115.3 \pm 1.0 \mathrm{GeV}$ & $115.3 \pm 0.2 \mathrm{GeV}$ \\
$M_{h q}^{\max }$ & Section 20.2.5 & $758.3 \pm 25 \mathrm{GeV}$ & $758.3 \pm 13 \mathrm{GeV}$ \\
\hline$M_{\tilde{q}_{R}}$ & Section 20.2.7.1 & $959 \pm 40 \mathrm{GeV}$ & $959 \pm 15 \mathrm{GeV}$ \\
$M_{\tilde{g}}$ & {$[20-23]$} & $1004 \pm 25 \mathrm{GeV}$ & $1004 \pm 12 \mathrm{GeV}$ \\
$M_{\tilde{i}_{1}}$ (Point 1) & {$[20-23]$} & none & $647 \pm 100 \mathrm{GeV}$ \\
$M_{\tilde{i}_{1}}$ (Point 2) & {$[20-23]$} & none & $713 \pm 100 \mathrm{GeV}$ \\
\hline
\end{tabular}


Table 20-6 Results of fits of the minimal SUGRA model to the measurements for Points 1 and 2 listed in Table 20-5.

\begin{tabular}{llll}
\hline Parameter & Low-L & High-L & Ultimate \\
\hline$m_{0}$ & $400 \pm 100 \mathrm{GeV}$ & $400 \pm 100 \mathrm{GeV}$ & $400 \pm 100 \mathrm{GeV}$ \\
$m_{1 / 2}$ & $400 \pm 10 \mathrm{GeV}$ & $400 \pm 8 \mathrm{GeV}$ & $400 \pm 8 \mathrm{GeV}$ \\
$\tan \beta$ (Point 1) & $2.00 \pm 0.08$ & $2.00 \pm 0.08$ & $2.00 \pm 0.02$ \\
$\tan \beta$ (Point 2) & $10.0 \pm 2.0$ & $10.0 \pm 2.0$ & $10.0 \pm 1.2$ \\
\hline
\end{tabular}

\subsubsection{Points 1 and 2}

Points 1 and 2 are high-mass points with relatively small cross sections. The decay $\tilde{\chi}_{2}^{0} \rightarrow \tilde{\chi}_{1}^{0} h$ is completely dominant, so there are fewer well-measured quantities than at some of the other points. These two points differ only in $\tan \beta$ and the corresponding light Higgs mass, so they will be discussed together. The measurements are summarised in Table 20-5 together with references to the appropriate sections of this document or supporting notes. In addition, it should be possible to measure $M_{h q}^{\min }$ as discussed in Section 20.2.6, but not enough work has been done to assign a reliable error. The measurements listed below the line in Table 20-5 do not measure the masses directly. For example, the measurement described in Section 20.2.7.1 measures a combination of the $\bar{q}_{R}$ and $\tilde{\chi}_{1}^{0}$ masses plus the production kinematics. Treating it as a measurement of a single mass is a reasonable first approximation and a necessary one to allow a scan of the whole parameter space to be made with moderate effort.

The results of the fit [20-27] are summarised in Table 20-6. The value of $m_{0}$ is not very well determined because sleptons do not occur in cascade decays at this point to any significant extent, and they are too heavy to be produced directly. Thus the only information on $m_{0}$ comes from the squark masses, which are dominated by $m_{1 / 2}$.

The determination of $\tan \beta$ comes mainly from the light Higgs mass, which also depends on sgn $\mu$. Figure 20-51 shows the light Higgs mass and the branching ratio for $\tilde{\chi}_{2}^{0} \rightarrow \tilde{\chi}_{1}^{0} Z$ as functions of $\tan \beta$ for both signs of $\mu$. Each curve consists of many points corresponding to choices of the other parameters that are consistent with all the measurements except the Higgs mass in Table 20-5. For Point 1 , the lower part of the figure shows that the Higgs mass determines two different values of $\tan \beta$ corresponding to the two signs of $\mu$. These values are indicated by the vertical lines in the upper half of the figure. Since the corresponding branching ratios for $\tilde{\chi}_{2}^{0} \rightarrow \tilde{\chi}_{1}^{0} Z$ are quite different, the two solutions can be distinguished by using the relative rates for $Z \rightarrow l l$ and $h \rightarrow b \bar{b}$.
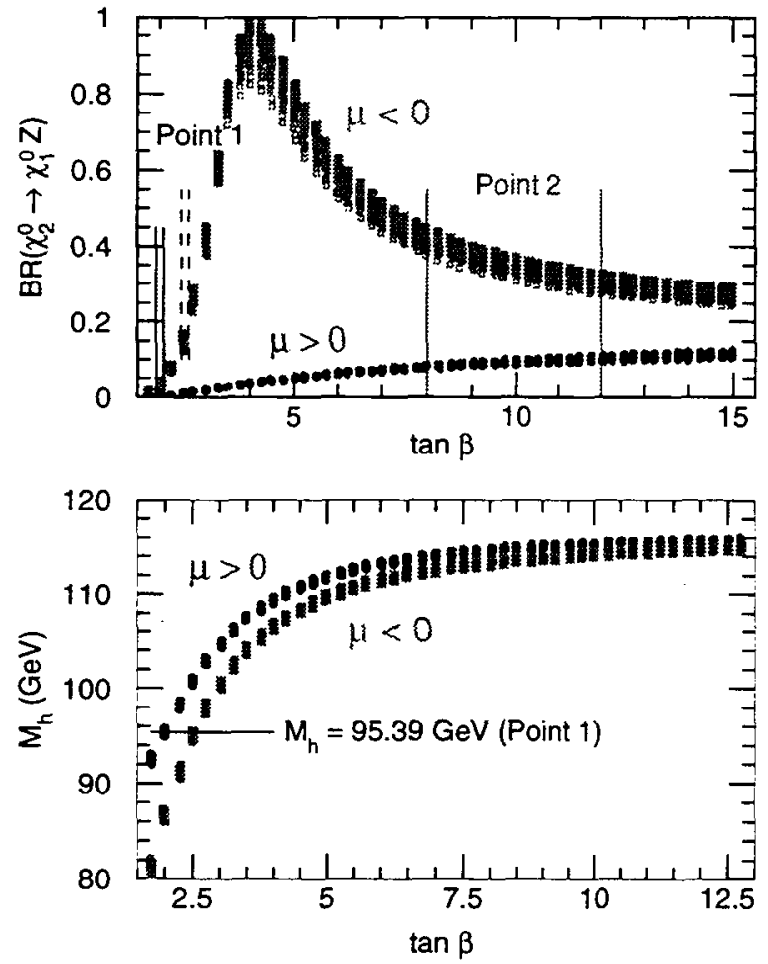

Figure 20-51 Higgs mass and $\tilde{\chi}_{2}^{0} \rightarrow \tilde{\chi}_{1}^{0} Z$ branching ratios as functions of $\tan \beta$ and sgn $\mu$ scanned over the allowed values of the other parameters. 
For Point 2, the Higgs mass has a much weaker dependence on $\tan \beta$ and is almost independent of $\operatorname{sgn} \mu$. The allowed region, again indicated by vertical lines, corresponds to different rates for $Z \rightarrow l l$ and $h \rightarrow b \bar{b}$. This can be used to determine sgn $\mu$, as was discussed in Section 20.2.5. Presumably these branching ratios could also be used in a more quantitative way to improve the measurement of $\tan \beta$ at Point 2, but this has not been studied.

\subsubsection{Point 3}

Point 3 is a low-mass point with many well-measured quantities. The best-measured ones are summarised in Table 20-7. The measurements listed below the line in the table actually determine combinations of masses. For example, the scatter plot shown in Section 20.2.4.1 really measures $M_{\bar{b}_{1}}-1.5 M_{\bar{\chi}_{1}^{0}}$ rather than $M_{\tilde{b}_{1}}$. Within the minimal SUGRA model, however, $M_{\bar{\chi}^{0}}$ is known since $M_{\tilde{\chi}_{2}^{0}}-M_{\tilde{\chi}_{1}^{0}}$ is accurately measured and the two gaugino masses are related by the GUT condition

$$
m_{1} \approx \frac{5}{3} \tan ^{2} \theta_{w} m_{2}
$$

Hence the distinction is less significant than for Points 1 and 2. While the fit is done including all the information simultaneously, $m_{1 / 2}$ is mainly determined by $M_{\bar{\chi}_{2}^{0}}-M_{\tilde{\chi}_{1}^{0}}$, which is accurately measured from the dilepton endpoint. Then $m_{0}$ is determined by measuring a squark mass using either $M_{\tilde{b}_{1}}$ or $M_{\tilde{g}}-M_{\tilde{b}_{1}}$. Finally, $\tan \beta$ is determined by the Higgs mass, which also fixes the sign of $\mu$. Given the tiny statistical errors for this low-mass point, it is therefore not surprising that all the parameters are accurately determined, as summarised in Table 20-8. The masses in this case are so light that all the errors are dominated by systematic effects, so there is little improvement with increasing luminosity.

Point 3 is of course somewhat unrepresentative, both because of its low masses and because of its unusual branching ratios. Detailed study of SUSY at the LHC will in general require the full luminosity available.

Table 20-7 Inputs for the minimal SUGRA model fit at Point 3. The measurements listed below the heavy rule really determine combinations masses and production dynamics.

\begin{tabular}{llll}
\hline Quantity & Reference & Low-L & Ultimate \\
\hline$M_{h}$ & Section 19.2.2 & $68.5 \pm 3.0 \mathrm{GeV}$ & $68.5 \pm 0.2 \mathrm{GeV}$ \\
$M_{\bar{\chi}_{2}^{0}}-M_{\tilde{\chi}_{1}^{0}}$ & Section 20.2.3 & $52.42 \pm 0.05 \mathrm{GeV}$ & $52.42 \pm 0.05 \mathrm{GeV}$ \\
$M_{\tilde{g}^{-}}-M_{\bar{b}_{1}}$ & Section 20.2.4.1 & $20.3 \pm 2.0 \mathrm{GeV}$ & $20.3 \pm 2.0 \mathrm{GeV}$ \\
\hline$M_{\tilde{b}_{1}}$ & Section 20.2 .4 .1 & $278.1 \pm 3.0 \mathrm{GeV}$ & $278.1 \pm 3.0 \mathrm{GeV}$ \\
$M_{\bar{q}_{L}}$ & Section 20.2 .4 .1 & $320.5 \pm 20.0 \mathrm{GeV}$ & $320.5 \pm 10.0 \mathrm{GeV}$ \\
\hline
\end{tabular}

Table 20-8 Results of a fit of the minimal SUGRA model to the measurements in Table 20-7.

\begin{tabular}{llll}
\hline Parameter & Low-L & High-L & Ultimate \\
\hline$m_{0}$ & $200.0 \pm 10.0 \mathrm{GeV}$ & $200.0 \pm 6.0 \mathrm{GeV}$ & $200.0 \pm 5.0 \mathrm{GeV}$ \\
$m_{1 / 2}$ & $100.0 \pm 1.0 \mathrm{GeV}$ & $100.0 \pm 1.0 \mathrm{GeV}$ & $100.0 \pm 1.0 \mathrm{GeV}$ \\
$\tan \beta$ & $2.00 \pm 0.05$ & $2.00 \pm 0.05$ & $2.00 \pm 0.02$ \\
\hline
\end{tabular}


Table 20-9 Inputs for the minimal SUGRA model fit at Point 4. The measurements below the horizontal rule really determine combinations of masses and production dynamics.

\begin{tabular}{llll}
\hline Quantity & Reference & Low-L & Ultimate \\
\hline$M_{h}$ & Section 19.2.2 & $111.8 \pm 1.0 \mathrm{GeV}$ & $111.8 \pm 0.2 \mathrm{GeV}$ \\
$M_{\tilde{\chi}_{2}^{0}}-M_{\tilde{\chi}_{1}^{0}}$ & Section 20.2.3 & $68.7 \pm 0.8 \mathrm{GeV}$ & $68.7 \pm 0.25 \mathrm{GeV}$ \\
$M_{\tilde{g}}-M_{\tilde{\chi}_{2}^{0}}$ & Section 20.2 .4 .2 & $434.0 \pm 12.0 \mathrm{GeV}$ & $434.0 \pm 6.0 \mathrm{GeV}$ \\
\hline$M_{\tilde{\chi_{2}^{I}}}$ & Section 20.2 .7 .2 & $315 \pm 20 \mathrm{GeV}$ & $315 \pm 7 \mathrm{GeV}$ \\
$\left\langle M_{\tilde{q}}\right\rangle$ & {$[20-25]$} & $915 \pm 25 \mathrm{GeV}$ & $915 \pm 25 \mathrm{GeV}$ \\
\hline
\end{tabular}

Table 20-10 Results of a fit of the measurements in Table 20-9 to the minimal SUGRA model.

\begin{tabular}{llll}
\hline Parameter & Low-L & High-L & Ultimate \\
\hline$m_{0}$ & $800 \pm 50 \mathrm{GeV}$ & $800 \pm 45 \mathrm{GeV}$ & $800 \pm 35 \mathrm{GeV}$ \\
$m_{1 / 2}$ & $200.0 \pm 4.0 \mathrm{GeV}$ & $200.0 \pm 2.0 \mathrm{GeV}$ & $200.0 \pm 1.5 \mathrm{GeV}$ \\
$\tan \beta$ & $10.0 \pm 2.0$ & $10.0 \pm 1.5$ & $10.0 \pm 0.6$ \\
\hline
\end{tabular}

\subsubsection{Point 4}

The squarks and sleptons are heavy at Point 4 because $m_{0}$ is very large. As a result, most of the measurements are controlled by gluino and gaugino masses, which have very little sensitivity to $m_{0}$. A number of possible methods to determine $m_{0}$ have been investigated [20-25]. The best approach seems to be to make a rough measurement of the squark mass by selecting events with at least five hard jets and large $E_{\mathrm{T}}^{\text {miss }}$. The fraction of events with a large value of

$$
E_{\mathrm{T}}^{\mathrm{sum}}=\sum_{i=1}^{5} p_{\mathrm{T}, i}
$$

is sensitive to the squark mass. The statistical errors are small; the main problem is to understand the systematic uncertainty on the relationship between the measured quantity and the squark mass. An estimate of this is included with the errors on the other measurements in Table 20-9.

The resulting fits for $\mu>0$ are given in Table 20-10. As expected, $m_{1 / 2}$ is well determined. Since the Higgs mass becomes insensitive to $\tan \beta$ for large values, the errors on it are sensitive to those assumed for the Higgs mass. The violation of $e / \mu / \tau$ universality in gaugino decays is still very small for $\tan \beta=10$, so the Higgs mass is probably the only way to determine $\tan \beta$ precisely. The errors on $m_{0}$ are larger and only improve slowly with increasing luminosity because the determination of the squark mass is limited by systematics. For low luminosity there is also a solution for $\mu<0$ with $m_{0}=820 \pm 50 \mathrm{GeV}, m_{1 / 2}=190 \pm 4 \mathrm{GeV}$, and $\tan \beta=12.5 \pm 1.5$, but this solution does not exist for high luminosity. For all fits $A_{0}$ is essentially undetermined for the reasons already explained. 
Table 20-11 Inputs for the minimal SUGRA model fit at Point 5 . The numerical values are obtained from the formulas given in the referenced sections plus masses from ISAJET 7.37.

\begin{tabular}{llll}
\hline Quantity & Reference & Low-L & Ultimate \\
\hline$M_{h}$ & Section 19.2 .2 & $92.9 \pm 1.0 \mathrm{GeV}$ & $92.9 \pm 0.2 \mathrm{GeV}$ \\
$M_{l l}^{\max }$ & Section 20.2 .3 & $108.92 \pm 0.50 \mathrm{GeV}$ & $108.92 \pm 0.10 \mathrm{GeV}$ \\
$M_{l q}^{\max }$ & Section 20.2 .4 .3 & $478.1 \pm 11.5 \mathrm{GeV}$ & $478.1 \pm 5.0 \mathrm{GeV}$ \\
$M_{l q}^{\max } / M_{l l q}^{\max }$ & Section 20.2 .4 .3 & $0.865 \pm 0.060$ & $0.865 \pm 0.020$ \\
$M_{l l q}^{\min }$ & Section 20.2 .6 & $271.8 \pm 14.0 \mathrm{GeV}$ & $271.8 \pm 5.4 \mathrm{GeV}$ \\
$M_{h q}^{\max }$ & Section 20.2 .5 & $552.5 \pm 10.0 \mathrm{GeV}$ & $552.5 \pm 5.5 \mathrm{GeV}$ \\
$M_{h q}^{\min }$ & Section 20.2 .6 & $346.5 \pm 17.0 \mathrm{GeV}$ & $346.5 \pm 17.0 \mathrm{GeV}$ \\
\hline
\end{tabular}

Table 20-12 Results of a fit [20-29] of the minimal SUGRA model to the measurements in Table 20-11.

\begin{tabular}{llll}
\hline Parameter & Low-L & High-L & Ultimate \\
\hline$m_{0}$ & $100.0_{-2.2}^{+4.1} \mathrm{GeV}$ & $100.0 \pm 1.4 \mathrm{GeV}$ & $100.0 \pm 1.3 \mathrm{GeV}$ \\
$m_{1 / 2}$ & $300.0 \pm 2.7 \mathrm{GeV}$ & $300.0 \pm 1.7 \mathrm{GeV}$ & $300.0 \pm 1.5 \mathrm{GeV}$ \\
$\tan \beta$ & $2.00 \pm 0.10$ & $2.00 \pm 0.09$ & $2.00 \pm 0.05$ \\
\hline
\end{tabular}

\subsubsection{Point 5}

At Point 5 there are two good starting points, $\tilde{\chi}_{2}^{0} \rightarrow \tilde{l}_{R} l$ and $\tilde{\chi}_{2}^{0} \rightarrow \tilde{\chi}_{1}^{0} h$, so there are many measurements that can be precisely expressed in terms of particle masses. These are summarised in Table 20-11 using masses from the same version of ISAJET that was used for the analyses described in Section 20.2.4.3 and Section 20.2.6. The statistical errors determined in those sections have been scaled appropriately with luminosity and then combined with the usual energy scale systematic errors.

The resulting fits [20-29] are shown in Table 20-12. All three parameters are well determined, but $A_{0}$ is still essentially undetermined despite all the precise measurements. This can be understood by examining the distribution shown in Figure 20-52 of the values of $A_{0}$ and of the weak scale parameters $A_{t}$ and $A_{b}$ allowed by the fit. Evidently the weak
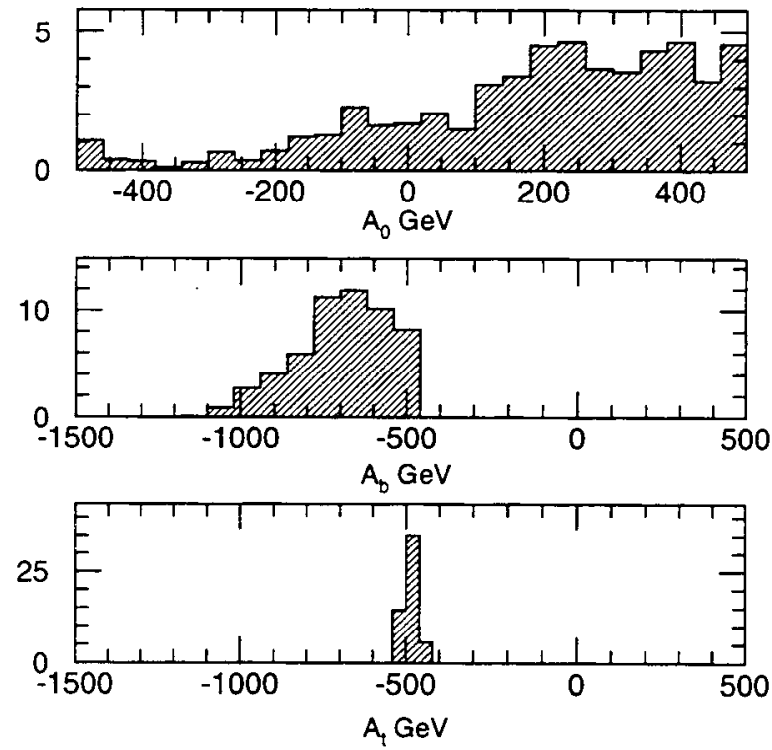

Figure 20-52 Allowed values of the GUT scale $A_{0}$ and the weak scale $A_{t}$ and $A_{b}$ for Point 5. scale parameters, which are what can actually be measured, are reasonably well determined but are simply insensitive to $A_{0}$. 
Table 20-13 Inputs for the minimal SUGRA model fit at Point 6 . The measurement listed below the heavy rule really determines a combination of masses and production dynamics; conservative (optimistic) errors for it are given.

\begin{tabular}{llll}
\hline Quantity & Reference & Low-L & Ultimate \\
\hline$M_{h}$ & Section 19.2 .2 & $111.9 \pm 1.0 \mathrm{GeV}$ & $111.9 \pm 0.2 \mathrm{GeV}$ \\
$M_{\tau \tau}^{\max }$ & Section 20.2 .8 .2 & $59.6 \pm 3.0 \mathrm{GeV}$ & $59.6 \pm 1.2 \mathrm{GeV}$ \\
$M_{\bar{g}}-M_{\tilde{b}_{1}}$ & Section 20.2 .8 .3 & $150 \pm 20 \mathrm{GeV}$ & $150 \pm 10 \mathrm{GeV}$ \\
$M_{\bar{g}}$ & Section 20.2 .8 .3 & $540 \pm 60 \mathrm{GeV}$ & $540 \pm 30 \mathrm{GeV}$ \\
\hline$M_{\tilde{q}_{R}}$ & Section 20.2 .8 .4 & $498 \pm 50(25) \mathrm{GeV}$ & $498 \pm 25(12) \mathrm{GeV}$ \\
\hline
\end{tabular}

Table 20-14 Results of a fit of the measurements in Table 20-13 to the minimal SUGRA model. There are solutions for both signs of $\mu$.

\begin{tabular}{llll}
\hline Parameter & Low-L & High-L & Ultimate \\
\hline$m_{0}$ & & $\mu<0$ & \\
$m_{1 / 2}$ & $236 \pm 37 \mathrm{GeV}$ & $242 \pm 28 \mathrm{GeV}$ & $242 \pm 25 \mathrm{GeV}$ \\
$\tan \beta$ & $200 \pm 14 \mathrm{GeV}$ & $196 \pm 10 \mathrm{GeV}$ & $194 \pm 6 \mathrm{GeV}$ \\
$A_{0}$ & $41 \pm 3.9$ & $44 \pm 1.7$ & $45 \pm 1.7$ \\
\hline & $0 \pm 180 \mathrm{GeV}$ & $-60 \pm 132 \mathrm{GeV}$ & $-50 \pm 80 \mathrm{GeV}$ \\
\hline$m_{0}$ & & $\mu>0$ & \\
$m_{1 / 2}$ & $228 \pm 39 \mathrm{GeV}$ & $237 \pm 26 \mathrm{GeV}$ & $218 \pm 30 \mathrm{GeV}$ \\
$\tan \beta$ & $200 \pm 15 \mathrm{GeV}$ & $197 \pm 9 \mathrm{GeV}$ & $196 \pm 8 \mathrm{GeV}$ \\
$A_{0}$ & $41 \pm 3.7$ & $44 \pm 1.7$ & $44 \pm 1.1$ \\
\hline
\end{tabular}

\subsubsection{Point 6}

The measurements discussed previously for Point 6 are summarised in Table 20-13. For the $\tilde{q}_{R}$ signature the error is almost entirely systematic, and the distribution shown in Figure 20-50 is featureless. Two error estimates are given in the table; the more conservative will be used except in the Ultimate fit. The errors at high luminosity for measurements involving $\tau$ 's assume that hadronic $\tau$ decays can be identified and measured in the presence of pileup. This was not studied in Reference [20-34], but in Section 9.1.5.4 it is shown that $\tau$ identification and measurement are not significantly affected by high luminosity running.

The fit [20-33] was carried out as before extending the $\tan \beta$ range to $1<\tan \beta<60$. The upper limit was set because larger values lead to a negative squared mass for the $\tilde{\tau}_{1}$, breaking electromagnetic gauge invariance; it does not restrict the fit. The results are given in Table 20-14. There are nearly equivalent solutions for both signs of $\mu$. This is to be expected: by a change of conventions, the sign of $\mu$ with $\tan \beta>0$ can be traded for the sign of $\tan \beta$ with $\mu>0$, and $\tan \beta \approx \pm \infty$ are equivalent [20-35]. The value of $A_{0}$ is also quite well determined, in contrast to 
the points with lower $\tan \beta$. While Point 6 is certainly difficult, it is surprising how much can be measured. In part this is due to the fact that the masses have to be low to avoid $\tilde{\chi}_{2}^{0} \rightarrow \bar{\chi}_{1}^{0} h$, leading to large event samples.

\subsubsection{Non-universal SUGRA models}

The fits in Section 20.2.9 all assume the minimal SUGRA parameters with just four real parameters plus $\operatorname{sgn} \mu$. While this simplicity is somewhat justified by the universality of gravity and by the need to satisfy limits based on precision low-energy tests, it is by no means necessary. Models of physics near the Planck scale can lead to qualitatively new signatures such as flavour mixing and $C P$ violation in the slepton sector [20-31]. For example, it is possible to have a substantial rate for $\tilde{\chi}_{2}^{0} \rightarrow \tilde{\mu}^{ \pm} e^{\mp} \rightarrow \tilde{\chi}_{1}^{0} \mu^{ \pm} e^{\mp}$ at the LHC while still satisfying existing bounds on $\mu \rightarrow e \gamma$ and $\mu \rightarrow e$ conversion in the field of a nucleus. It is clear from Section 20.2.3 that this particular signature could be detected by ATLAS with good sensitivity.

A completely general SUGRA model has as many weak-scale parameters as the MSSM, so a general exploration is not possible without some experimental guidance. Since low-energy tests severely constrain flavour mixing and $C P$-violating phases, these are assumed to be absent, although there is no good justification for this assumption. This still leaves nineteen real parameters plus $\operatorname{sgn} \mu$, corresponding to the parameters of ISAJET. In this first exploration, three possible deviations from universality at the GUT scale have been considered: non-universal Higgs masses, non-universal 5 and 10 sfermion masses, and non-universal third generation masses. Each case can be characterised by one additional parameter. In general these new parameters are less well constrained than the minimal ones. Only exploratory work was performed on a few of the many possible variants of the model. Due to the preliminary nature of the work, in most cases no explicit numerical conclusion is drawn, but it is shown that in all considered cases ATLAS is sensitive to the model parameters.

\subsubsection{Nonuniversal Higgs masses}

Additional Higgs bosons, some of them superheavy, are needed to break the GUT gauge group down to $S U(3) \times S U(2) \times U(1)$, so it makes sense to distinguish the Higgs bosons from the squarks and sleptons. For this case, the soft Higgs masses are taken to be equal but different from those of the other scalars at the GUT scale:

$$
\begin{gathered}
m_{H_{u}}=m_{H_{d}}=m_{0, H} \\
m_{\tilde{Q}}=m_{\tilde{q}_{R}}=m_{\tilde{L}}=m_{\tilde{l}_{R}}=m_{0}
\end{gathered}
$$

The measurements for Point 5 were refit including $m_{0, H}^{2}>0$ in addition to the minimal SUGRA parameters. For the five-parameter fit, the error on $m_{1 / 2}$ is about $20 \%$ larger, while the errors on $m_{0}$ and $\tan \beta$ are almost the same [20-29]. However, the new parameter is poorly constrained,

$$
m_{0, H}<400 \mathrm{GeV}(95 \% \mathrm{CL}) \quad \text {. }
$$

The fit is insensitive to the new parameter because the derived value of $\mu$ is large, about $500 \mathrm{GeV}$. The value of $M_{H_{\mu}}$ at the weak scale is determined through the RGE's mainly by the top Yukawa coupling and the $t$ mass, not by $M_{H_{u}}$ at the GUT scale. As a result the masses of the $\tilde{\chi}_{1}^{0}$, $\tilde{\chi}_{2}^{0}$, and $h$ have little dependence on $m_{0, H}$ unless it is of order the heavy mass scale. 
To determine $m_{0, H}$ it is necessary to measure the masses of the heavy Higgs bosons $H$ and $A$, which vary by about $40 \mathrm{GeV}$ over this range of $m_{0, H}$. Since at Point 5 these have small production cross sections and decay into $t \dot{t}$ more than $90 \%$ of the time, it seems difficult to detect them at the LHC.

\subsubsection{Non-universal $\underline{5}$ and $\underline{10}$ sfermion masses}

The second non-universal SUGRA possibility considered here is that the sfermions in the 5 and 10 representations of $S U(5)$ might have different masses:

$$
\begin{gathered}
m_{\tilde{d}_{R}}=m_{\tilde{L}}=m_{0,5} \\
m_{\tilde{Q}}=m_{\tilde{u}_{R}}=m_{\tilde{l}_{R}}=m_{H_{u}}=m_{H_{d}}=m_{0}
\end{gathered}
$$

If the fit is redone for this case using only the information in Table 20-11, then only a poor limit is obtained on the new parameter:

$$
m_{0,5}<390 \mathrm{GeV}(95 \% \text { C.L.) ; }
$$

the small error on $m_{1 / 2}$ about doubles and the errors on $m_{0}$ and $\tan \beta$ increase slightly. The reason for this poor limit is that only one of the four squarks of each generation is in the $\underline{5}$, and its mass is dominated by $m_{1 / 2}$.

The constraint on $m_{0,5}$ can be improved by using more information. If $m_{0,5} \leq 85 \mathrm{GeV}$, then the decay $\tilde{\chi}_{2}^{0} \rightarrow \tilde{l}_{L} l \rightarrow \tilde{\chi}_{1}^{0} l l$ is kinematically allowed and produces a second edge in the dilepton mass distribution at

$$
M_{l l}^{\max }=M_{\tilde{\chi}_{2}^{0}} \sqrt{1-\frac{M_{\bar{l}_{L}}^{2}}{M_{\tilde{\chi}_{2}^{0}}^{2}}} \sqrt{1-\frac{M_{\tilde{\chi}_{1}^{0}}^{2}}{M_{\tilde{l}_{L}}^{2}}}
$$

Near threshold, one of the leptons is very soft, so a low $p_{\mathrm{T}}$ threshold is essential. A sample of 200000 events was generated with ISAJET for $m_{0,5}=75 \mathrm{GeV}$ [20-29]. Events were selected using the same jet and missing energy cuts as for the Point 5 analysis in Section 20.2 .3 but requiring two muons with $p_{\mathrm{T}}>5 \mathrm{GeV}$. The resulting $\mu^{+} \mu^{-}$mass distribution, Figure 20-53, shows a clear second edge with an integrated luminosity of $10 \mathrm{fb}^{-1}$. This edge is almost invisible in the $e^{+} e^{-}$mass distribution with the standard cut $p_{\Upsilon}>10 \mathrm{GeV}$.

The slepton mass for $m_{0,5}=75 \mathrm{GeV}$ differs from that for Point 5 by only $9 \mathrm{GeV}$. It would not be easy to distinguish these using the direct slepton production analysis described in Section 20.2.7.4 even with an integrated luminosity of $300 \mathrm{fb}^{-1}$. It should be possible at high

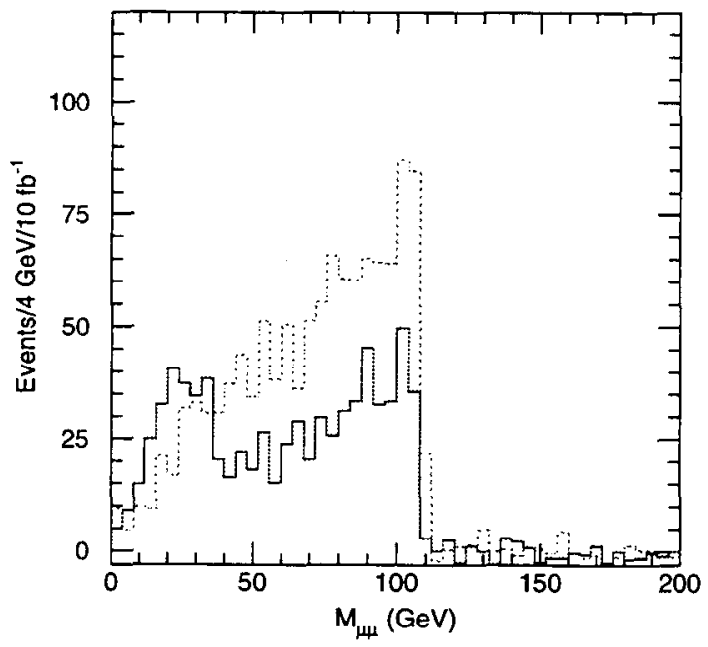

Figure 20-53 $\mu^{+} \mu^{-}$mass distribution for modified Point 5 with $m_{0,5}=75 \mathrm{GeV}$ (solid) and for Point 5 (dashed). 
luminosity to use that analysis to distinguish $m_{0,5}=150 \mathrm{GeV}$, for which the $\tilde{l}_{L}$ is $24 \mathrm{GeV}$ heavier than at Point 5. The rate of course becomes smaller for larger $m_{0,5}$; at some point one must rely on the absence of a direct slepton signal.

Direct $\tilde{l}_{L}^{+} \tilde{l}_{L}^{-}$production can also give a four-lepton signature if one slepton decays via $\tilde{l}_{L} \rightarrow \bar{\chi}_{1}^{0} l$ and the other via $\tilde{l}_{L} \rightarrow \tilde{\chi}_{2}^{0} l \rightarrow \tilde{l}_{L}^{\prime} l^{\prime} l \rightarrow \tilde{\chi}_{1}^{0} l^{\prime} l^{\prime} l$. This branching ratio is too small to be observable at Point 5 but increases for larger $m_{0,5}$. One virtue of this signature is that the three-lepton mass has a kinematic endpoint at

$$
M_{l l l}^{\max }=\sqrt{1-\frac{M_{\tilde{l}_{R}}^{2}}{M_{\tilde{\chi}_{2}^{0}}^{2}}} \sqrt{M_{\bar{l}_{L}}^{2}\left(1-\frac{M_{\bar{\chi}_{2}^{0}}^{2}}{M_{l_{L}}^{2}}\right)+M_{\tilde{\chi}_{2}^{0}}^{2}\left(1-\frac{M_{\tilde{\chi}_{1}^{0}}^{2}}{M_{l_{R}}^{2}}\right)}=128.0 \mathrm{GeV}, \quad m_{0,5}=125 \mathrm{GeV} .
$$

A sample of 200000 events was generated [2029] with $m_{0,5}=125 \mathrm{GeV}$ and the other parameters the same as for Point 5 . The main background comes from the strong production of gluinos and squarks, so a jet veto is required even with four leptons. The following cuts were made:

- no jet with $p_{\mathrm{T}}>40 \mathrm{GeV}$ and $|\eta|<5$;

- at least four isolated leptons forming two opposite sign, same flavour pairs;

- $M_{l l}<108 \mathrm{GeV}$ for at least one opposite sign, same flavour pair to be consistent with a $\tilde{\chi}_{2}^{0}$ decay.

After these cuts, the pair with $M_{l l}<108 \mathrm{GeV}$ was combined with the lowest $p_{\mathrm{T}}$ additional lepton, since there is much more phase space

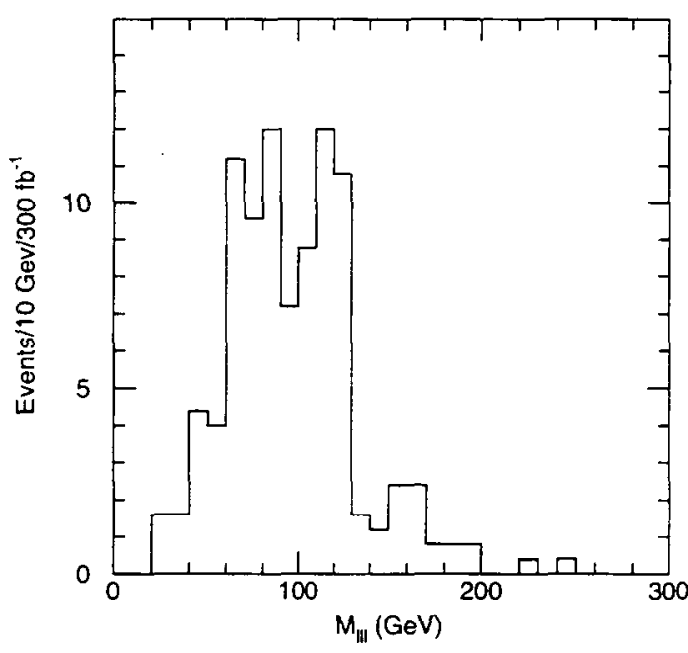

Figure 20-54 Three-lepton mass distribution in clean four-lepton events at Point 5 with $m_{0,5}=125 \mathrm{GeV}$. for $\tilde{l}_{L} \rightarrow \tilde{\chi}_{1}^{0} l$ than for $\tilde{l}_{L} \rightarrow \tilde{\chi}_{2}^{0} l$. The resulting three-lepton mass distribution is shown in Figure 20-54 for an integrated luminosity of $300 \mathrm{fb}^{-1}$. The number of events is small even for the full luminosity of the LHC, but an endpoint at about the right position can be seen. Combining this signature with the two-lepton direct production signature would provide sensitivity to $m_{0,5} \approx 125 \mathrm{GeV}$, much less than the limit from fitting only the measurements in Table 20-11.

\subsubsection{Non-universal third generation masses}

The third possibility considered here is that the sfermions of the third generation have different masses at the GUT scale:

$$
m_{\tilde{t}_{L, R}}=m_{\tilde{b}_{L, R}}=m_{\tilde{\tau}_{L, R}}=m_{0,3},
$$

with the rest of the scalar masses being $m_{0}$. Since the $\tilde{t}_{1,2}$ and $\tilde{b}_{1,2}$ masses are dominated by $m_{1 / 2}$, the main effect of changing $m_{0,3}$ is on the $\tilde{\tau}_{1,2}$ masses.

It is possible in principle to obtain information on the $\tilde{\tau}_{1}$ mass by measuring the visible $\tau \tau$ mass distribution, as was discussed for Point 6 in Section 20.2.8.2. For Point 5, however, the branching ratio for $\bar{\chi}_{2}^{0} \rightarrow \tilde{\tau} \tau$ is only $11.8 \%$, opposed to $100 \%$ for Point 6 , so extracting a $\tau \tau$ endpoint is 
considerably more difficult than it is at Point 6 . The signal seems to be lost in the fluctuations of the backgrounds from other sources [20-29]. If $\tan \beta$ is increased somewhat, then the $\tilde{\chi}_{2}^{0} \rightarrow \tilde{\chi}_{1}^{0} h$ mode is closed, the slepton modes dominate, and a signal can be extracted.

It is easier, although less direct, to get information on the $\tau \tau$ distribution by counting the number of like-flavour and unlike-flavour dileptons. Events were selected with

- $E_{\mathrm{T}}^{\text {miss }}>\max \left(0.2 M_{\text {eff }}, 100 \mathrm{GeV}\right)$;

- at least four $R=0.4$ jets with $p_{\mathrm{T}}>100,50,50,50 \mathrm{GeV}$;

- two isolated electrons or muons with $p_{\mathrm{T}}>10 \mathrm{GeV}$ and $|\eta|<2.5$.

The ratio of same-flavour to opposite-flavour dileptons with opposite sign,

$$
R_{+-}=\frac{N\left(e^{+} e^{-}\right)+N\left(\mu^{+} \mu^{-}\right)}{N\left(e^{ \pm} \mu^{\mp}\right)}
$$

after these cuts is given in Table 20-15 for several values of $m_{0,3}$. This ratio is equal to one for two $\tau$ decays or for any other combination of two independent decays satisfying $e-\mu$ universality; it is greater than one for flavourcorrelated decays such as $\tilde{\chi}_{2}^{0} \rightarrow \tilde{l} l$. Since the $\tilde{\tau}_{1}$ is lighter for low values of $m_{0,3}$, the decay $\tilde{\chi}_{2}^{0} \rightarrow \tilde{\tau} \tau$ is enhanced, reducing $R_{+-}$. For $m_{0,3}=150 \mathrm{GeV}$ the $\tilde{\tau}_{1}$ mass is $193 \mathrm{GeV}$. In this region the two-body decay $\tilde{\chi}_{2}^{0} \rightarrow \tilde{\tau} \tau$ is not allowed; the $\tau$ branching ratios are determined by an interference between virtual slepton and $Z$ exchange. Of course, the ratio $R_{+}$. is also sensitive to $\tan \beta$, so this measurement must be combined with other information such as the Higgs mass before any conclusion can be drawn.

It would also be useful to obtain a measurement of single $\tau$ production by counting the number of leptons and hadronic $\tau$ decays. Since the jet multiplicity is substantial, this requires an algorithm for selecting hadronic $\tau$ 's optimised for purity rather than for visible mass resolution as in Section 20.2.8. Presumably tight isolation cuts together with a displaced vertex should be required. This has not been studied in the framework of SUSY studies, but work along these lines is documented in Section 9.1.5.2

\subsection{Gauge mediated SUSY breaking models}

In Gauge Mediated SUSY Breaking (GMSB) models [20-13], SUSY breaking occurs in a separate sector, as in SUGRA models, but at a much lower scale, $F_{m}$ " $\left(10^{10} \mathrm{GeV}\right)^{2}$. It is then communicated to the MSSM particles through ordinary $S U(3) \times S U(2) \times U(1)$ gauge interactions at a messenger scale that is much lower than the Planck scale, $M_{m} \ll M_{P}$, and perhaps even close to the weak scale. This implies that all MSSM particles with the same Standard Model quantum numbers have the same mass at the scale $M_{m}$, so flavour changing neutral currents are suppressed. Since the gravitino $\tilde{G}$ gets its mass only through gravitational couplings at $M_{P}$, it is 
much lighter than the $R=-1$ MSSM particles - generally much lighter than $1 \mathrm{GeV}$. The helicity- $1 / 2$ couplings of the gravitino are enhanced by factors of $1 / M_{\tilde{G}}$, so all the other SUSY particles eventually decay into it.

The successful unification of coupling constants in the MSSM [20-12] is preserved if the messenger fields are chosen to be complete vector-like representations of $S U(5), e . g ., 5+\overline{5}$ or $10+\overline{10}$. In the minimal GMSB model considered here this is assumed, but the number $N_{5}$ of equivalent $5+\overline{5}$ representations is left arbitrary. A single $10+\overline{10}$ gives $N_{5}=3$; a combination of several representations can produce a non-integer effective $N_{5}$. It is also assumed that electroweak symmetry is broken radiatively through the large top Yukawa coupling as in minimal SUGRA model and that $\mu$ and $B \mu$ are generated. Finally, the gravitino can get mass not just from the messenger sector but from any other source of SUSY breaking, presumably in some more complex hidden sector. Then the parameters of the minimal GMSB model are [20-13]

$$
\Lambda \equiv F_{m} / M_{m}, \quad M_{m}, \quad N_{5}, \quad \tan \beta, \quad \operatorname{sgn} \mu= \pm 1, \quad C_{\text {grav }},
$$

where $C_{\text {grav }} \geq 1$ is the ratio of the gravitino mass to its value if the only source of SUSY breaking is $F_{m}$; lifetimes for gravitino decay are proportional to $C_{\mathrm{grav}}^{2}$. Gauginos get masses at one loop at the messenger scale, and scalars get squared masses at two loops, e.g.

$$
\begin{aligned}
M_{\tilde{g}} & =\frac{\alpha_{s}}{4 \pi} \Lambda N_{5} \\
M_{\bar{e}_{L}}^{2} & =\frac{3 \alpha_{2}^{2}}{32 \pi^{2}} \Lambda^{2} N_{5}+\frac{3 \alpha_{1}^{2}}{160 \pi^{2}} \Lambda^{2} N_{5} \\
M_{\bar{e}_{R}}^{2} & =\frac{3 \alpha_{1}^{2}}{40 \pi^{2}} \Lambda^{2} N_{5} \\
M_{\bar{u}_{L}}^{2} & =\frac{\alpha_{s}^{2}}{6 \pi^{2}} \Lambda^{2} N_{5}+\frac{3 \alpha_{2}^{2}}{32 \pi^{2}} \Lambda^{2} N_{5}+\frac{\alpha_{1}^{2}}{480 \pi^{2}} \Lambda^{2} N_{5} \\
M_{\tilde{u}_{R}}^{2} & =\frac{\alpha_{s}^{2}}{6 \pi^{2}} \Lambda^{2} N_{5}+\frac{\alpha_{1}^{2}}{30 \pi^{2}} \Lambda^{2} N_{5}
\end{aligned}
$$

Note that the gaugino and scalar masses are comparable, but gaugino masses are proportional to $N_{5}$ while scalar masses are proportional to $\sqrt{N_{5}}$.

In GMSB models the lightest SUSY particle is the gravitino with $M_{\tilde{G}}$ « $1 \mathrm{GeV}$. The Next Lightest SUSY Particle (NLSP) is generally the $\tilde{\chi}_{1}^{0}$ if $N_{5}=1$ and a right-handed slepton if $N_{5}>1$, since these particles only have masses proportional to the small $\alpha_{1}$ coupling. If $C_{\mathrm{grav}} \sim 1$, the NLSP will decay promptly to gravitinos via $\tilde{\chi}_{1}^{0} \rightarrow \tilde{G} \gamma$ or $\tilde{l}_{R} \rightarrow \tilde{G} l$, while if $C_{\text {grav }}$ 1 1 the NLSP will decay mainly outside the detector. (The $\tilde{l}_{R}$ must of course not be stable over the lifetime of the universe.) Thus there are four distinct cases for GMSB phenomenology, depending on whether the NLSP is a neutralino or a slepton and whether it has a short or a long lifetime.

One minimal GMSB model point for each case was selected for detailed study [20-36], forming two pairs differing only by the value of $C_{\mathrm{grav}}$ and hence by the lifetime of the NLSP. The parameters of these points are listed in Table 20-16; the corresponding masses from ISAJET [20-15] are given in Table 20-17. These points illustrate the main features expected in GMSB models: Point G1a gives events with two hard photons plus $E_{T}^{\text {miss }}$; Point G1b gives signatures qualitatively like SUGRA models; Point G2a gives multiple leptons; and Point G2b gives quasi-stable charged sleptons. Since the signatures are so different, each point will be discussed separately. Most of the results in this section are based on ISAJET and a simple particle-level simulation of 
Table 20-16 Parameters of the four GMSB points considered in Section 20.3.

\begin{tabular}{lllllll}
\hline Point & $\Lambda(\mathrm{TeV})$ & $M_{m}(\mathrm{TeV})$ & $N_{5}$ & $\tan \beta$ & $\operatorname{sgn} \mu$ & $C_{\text {grav }}$ \\
\hline G1a & 90 & 500 & 1 & 5.0 & + & 1.0 \\
G1b & 90 & 500 & 1 & 5.0 & + & $10^{3}$ \\
G2a & 30 & 250 & 3 & 5.0 & + & 1.0 \\
G2b & 30 & 250 & 3 & 5.0 & + & $5 \times 10^{3}$ \\
\hline
\end{tabular}

Table 20-17 Masses in GeV for the particles at the GMSB points in Table 20-16 from ISAJET 7.37 [20-15]. Only the gravitino mass depends on $C_{\text {grav }}$.

\begin{tabular}{|c|c|c|c|c|c|c|c|c|}
\hline Particle & Point G1 & Point G2 & Particle & Point G1 & Point G2 & Particle & Point G1 & Point G2 \\
\hline$\tilde{g}$ & 747 & 713 & $\tilde{u}_{L}$ & 986 & 672 & $\tilde{e}_{L}$ & 326 & 204 \\
\hline$\tilde{\chi}_{1}^{ \pm}$ & 223 & 201 & $\tilde{u}_{R}$ & 942 & 649 & $\bar{e}_{R}$ & 164 & 103 \\
\hline$\tilde{\chi}_{2}^{ \pm}$ & 469 & 346 & $\tilde{d}_{L}$ & 989 & 676 & $\tilde{v}_{e}$ & 317 & 189 \\
\hline$\tilde{\chi}_{1}^{0}$ & 119 & 116 & $\tilde{d}_{R}$ & 939 & 648 & $\tilde{\tau}_{1}$ & 163 & 102 \\
\hline$\tilde{\chi}_{2}^{0}$ & 224 & 204 & $\tilde{t}_{1}$ & 846 & 584 & $\tilde{\tau}_{2}$ & 326 & 204 \\
\hline$\tilde{\chi}_{3}^{0}$ & 451 & 305 & $\tilde{t}_{2}$ & 962 & 684 & $\tilde{v}_{\tau}$ & 316 & 189 \\
\hline \multirow[t]{4}{*}{$\tilde{\chi}_{4}^{0}$} & 470 & 348 & $\tilde{b}_{1}$ & 935 & 643 & $h$ & 110 & 107 \\
\hline & & & $\tilde{b}_{2}$ & 945 & 642 & $H$ & 557 & 360 \\
\hline & & & & & & $A$ & 555 & 358 \\
\hline & & & & & & $H^{ \pm}$ & 562 & 367 \\
\hline
\end{tabular}

the ATLAS detector. It appears that the backgrounds are dominated by irreducible physics processes, so that the detector performance is not critical. Detailed GEANT-based studies have been performed for non-pointing photons at Point G1a and for quasi-stable sleptons at Point G2b.

\subsubsection{GMSB Point G1a}

At Point Gla the total SUSY cross section is $7.6 \mathrm{pb}$. The NLSP is the $\tilde{\chi}_{1}^{0}$, and it decays primarily to $\tilde{G} \gamma$ with $c \tau=1.2 \mathrm{~mm}$. SUSY events are therefore characterised by two hard isolated photons plus the usual jets, leptons, and missing transverse energy $E_{T}^{\text {miss }}$ from the gravitinos and perhaps from neutrinos. The presence of two photons in almost every event renders the Standard Model backgrounds negligible and makes discovery trivial. In a small fraction $(2.0 \%)$ of the events, the NLSP will undergo a Dalitz decay to $\tilde{G} e^{+} e^{-}$. The $e^{+}$and $e^{-}$can be used to determine the decay vertex and so to make a precise measurement of the mean decay length. The mass of the $\tilde{\chi}_{1}^{0}$ can be determined as described in Section 20.3.1.1. If the polarisation of the $\tilde{\chi}_{1}^{0}$ can be neglected, as it is in the existing event generators, then its momentum distribution can be inferred from that of the photons and its proper lifetime determined. The polarisation is likely to be small since many channels contribute, but it has not been studied and could be the dominant uncertainty. This lifetime measurement is very important as it provides the only constraint on the true scale of SUSY breaking in all hidden sectors, not just the messenger sector.

For all the analyses in this subsection [20-36] events were selected to have: 
- $M_{\text {eff }}>400 \mathrm{GeV}$;

- $E_{\mathrm{T}}^{\mathrm{miss}}>0.1 M_{\mathrm{eff}}$;

- two photons with $p_{\mathrm{T}}>20 \mathrm{GeV}$ and $|\eta|<2.5$;

- at least two leptons with $p_{\mathrm{T}}>20 \mathrm{GeV}$ and $|\eta|<2.5$ for electrons and $p_{\mathrm{T}}>5 \mathrm{GeV}$ and $|\eta|<2.5$ for muons.

Jets were found using an $R=0.4$ cone and requiring more than one charged track with $p_{\mathrm{T}}>1 \mathrm{GeV}$ to avoid counting $\tau$ 's as jets. These cuts make the Standard Model background negligible; it remains so even for a $\gamma /$ jet rejection of only $10^{-3}$, much worse than is expected and is needed for $h \rightarrow \gamma \gamma$. Modes without leptons might of course also be useful.

\subsubsection{Lepton and photon distributions}

Events were selected with two photons and exactly two leptons satisfying the cuts listed above. Since GMSB models ensure flavour conservation, the leptons from

$\tilde{\chi}_{2}^{0} \rightarrow \tilde{l}^{ \pm} l^{\mp} \rightarrow \tilde{\chi}_{1}^{0} l^{+} l^{-} \rightarrow \tilde{G} l^{+} l^{-\gamma}$

must be correlated in flavour, so the flavour subtracted combination $e^{+} e^{-}+\mu^{+} \mu^{-}-e^{ \pm} \mu^{\mp}$ shown in Figure 20-55 selects this signal and removes both the SUSY and the small Standard Model background from two independent leptonic decays. This gives a dilepton mass distribution similar to that found for SUGRA Point 5 in Section 20.2.3 with a very sharp endpoint at

$$
\begin{aligned}
M_{l l}^{\max } & =M_{\bar{\chi}_{2}^{0}} \sqrt{1-\frac{M_{l_{R}}^{2}}{M_{\tilde{\chi}_{2}^{0}}^{2}}} \sqrt{1-\frac{M_{\tilde{\chi}_{1}^{0}}^{2}}{M_{l_{R}}^{2}}} . \\
& =105.1 \mathrm{GeV}
\end{aligned}
$$

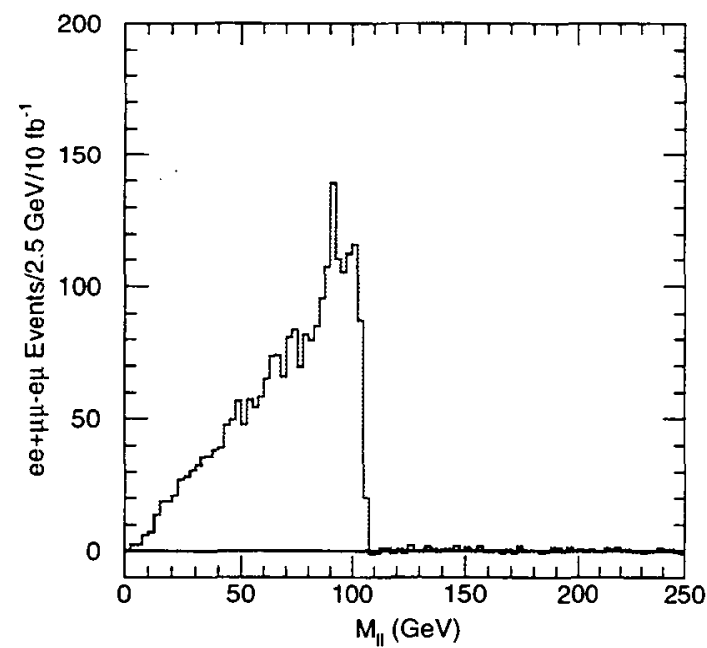

Figure 20-55 Flavour-subtracted dilepton distribution at Point G1a. The Standard Model background is not visible.

The other allowed two-body decay, $\tilde{\chi}_{2}^{0} \rightarrow \tilde{\chi}_{1}^{0} Z$, produces a small peak just visible in the figure. This does not directly measure masses and is not used here. It would be measurable with more luminosity, and the rate for it would then imply information about the Higgsino content of the two lightest neutralinos.

It is also useful to consider the whole 4-body decay chain, just as for the analysis of squark decay at Point 5 in Section 20.2.4.3. In this case the particles are leptons and photons, so the precision is much better. The sequence of three two-body decays implies that the $l^{+} l^{-} \gamma$ mass has an endpoint with a linear vanishing at

$$
M_{l l \gamma}^{\max }=\sqrt{M_{\bar{\chi}_{2}^{0}}^{2}-M_{\tilde{\chi}_{1}^{0}}^{2}}=189.7 \mathrm{GeV} .
$$




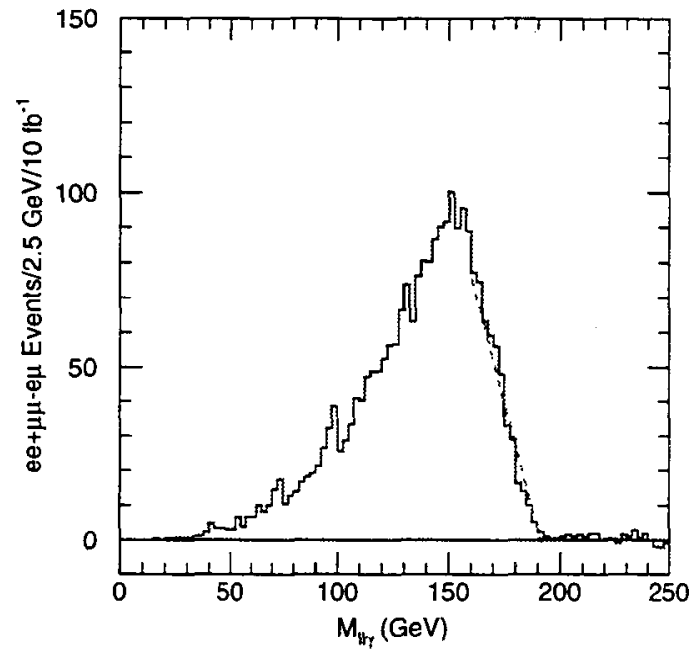

Figure 20-56 Flavour-subtracted distribution for the smaller $l^{+} l^{-} \gamma$ mass at Point G1a.

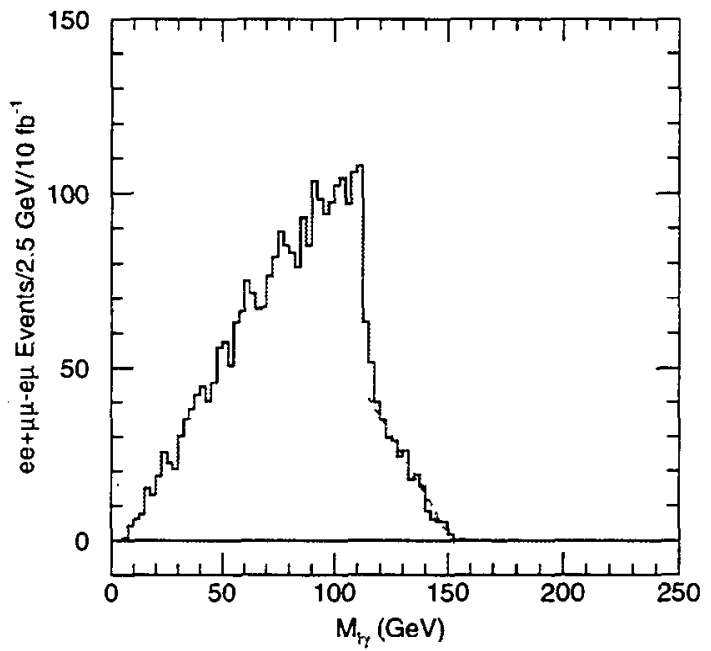

Figure 20-57 Flavour-subtracted $l^{ \pm} \gamma$ distribution for the $l^{+} l^{-} \gamma$ combination below its endpoint.

Since at least one of the $l^{+} l^{-} \gamma$ masses must be less than this, the distribution of the smaller one vanishes at this point. The flavour-subtracted distribution for the smaller $l^{+} l^{-} \gamma$ mass is shown in Figure 20-56 together with a linear fit near the endpoint. The endpoint could be measured to about $\pm 0.5 \mathrm{GeV}$ with $30 \mathrm{fb}^{-1}$ and to the systematics limit of about $\pm 0.2 \mathrm{GeV}$ with $300 \mathrm{fb}^{-1}$. The $l^{ \pm} \gamma$ mass distribution from $\tilde{\chi}_{2}^{0}$ decays has a sharp edge like the dilepton one from pairing the photon and the 'right' lepton - that is, the second one, which is adjacent to the photon in the decay chain - at

$$
M_{l \gamma}^{(1)}=\sqrt{M_{\tilde{l}_{R}}^{2}-M_{\tilde{\chi}_{1}^{0}}^{2}}=112.7 \mathrm{GeV}
$$

plus an endpoint with a linear vanishing from pairing the photon and the 'wrong' lepton at

$$
M\rangle_{\gamma}^{2)}=\sqrt{M_{\bar{\chi}_{2}^{0}}^{2}-M_{l_{R}}^{2}}=152.6 \mathrm{GeV}
$$

In contrast to the squark case, the second endpoint is greater than the first one and so is also visible. Events were selected to have the mass of one $l^{+} l^{-} \gamma$ combination less than and the other greater than $189.7 \mathrm{GeV}$, so that only one combination is consistent with $\tilde{\chi}_{2}^{0}$ decay. The $l^{ \pm} \gamma$ mass distribution for this $l^{+} l^{-} \gamma$ combination is shown in Figure 20-57. The errors on this edge and endpoint are estimated to be $\pm 0.2 \mathrm{GeV}$ and $\pm 0.5 \mathrm{GeV}$ with $30 \mathrm{fb}^{-1}$, reducing to $\pm 0.1 \mathrm{GeV}$ and $\pm 0.2 \mathrm{GeV}$ with $300 \mathrm{fb}^{-1}$.

The four measurements just described are sufficient to determine the $\tilde{\chi}_{2}^{0}, \tilde{l}_{R}$, and $\tilde{\chi}_{1}^{0}$ masses without any assumptions except for the neglect of the gravitino mass:

$$
\begin{aligned}
M_{l_{R}}^{2} & =\frac{\left(M_{l_{\gamma}}^{(1)}\right)^{2}\left(M_{l \gamma}^{(2)}\right)^{2}}{\left(M_{l l}^{\max }\right)^{2}} \\
M_{\tilde{\chi}_{1}^{0}}^{2} & =M_{l_{R}}^{2}-\left(M_{l \gamma}^{(1)}\right)^{2} \\
M_{\tilde{\chi}_{2}^{0}}^{2} & =M_{l_{R}}^{2}+\left(M_{l \gamma}^{(2)}\right)^{2}
\end{aligned}
$$


with the evident constraint

$$
\left(M_{l l \gamma}^{\max }\right)^{2}=\left(M_{l \gamma}^{(1)}\right)^{2}+\left(M_{l \gamma}^{(2)}\right)^{2}
$$

This result demonstrates the power of identifying and using a multi-step decay chain even more clearly than the $\vec{q}_{L}$ analysis at SUGRA Point 5 , since in this case only leptons and photons are involved. Of course the rates and hence the errors are model dependent, as is the interpretation of the slepton as a $\tilde{l}_{R}$.

\subsubsection{Reconstruction of $\tilde{G}$ momenta}

Once the masses have been determined, the decay $\tilde{\chi}_{2}^{0} \rightarrow \tilde{l}^{ \pm} l^{\mp} \rightarrow \tilde{\chi}_{1}^{0} l^{+} l^{-} \rightarrow \tilde{G} l^{+} l^{-} \gamma$ provides three mass constraints and hence a $0 C$ fit for the gravitino momentum $p$,

$$
\begin{gathered}
\left(p+p_{\gamma}\right)^{2}=M_{\tilde{\tilde{\chi}}_{1}^{0}}^{2} \\
\left(p+p_{\gamma}+p_{l_{2}}\right)^{2}=M_{l_{R}}^{2} \\
\left(p+p_{\gamma}+p_{l_{2}}+p_{l_{1}}\right)^{2}=M_{\tilde{\chi}_{2}^{0}}^{2}
\end{gathered}
$$

assuming that the gravitino mass $p^{2}$ can be neglected. There is a two-fold ambiguity from assigning the order of the leptons - i.e., which one appears in the second equation above and another two-fold ambiguity from solving a quadratic equation. If this decay chain occurs twice in the same event, both gravitino momenta can be determined, and the best so-

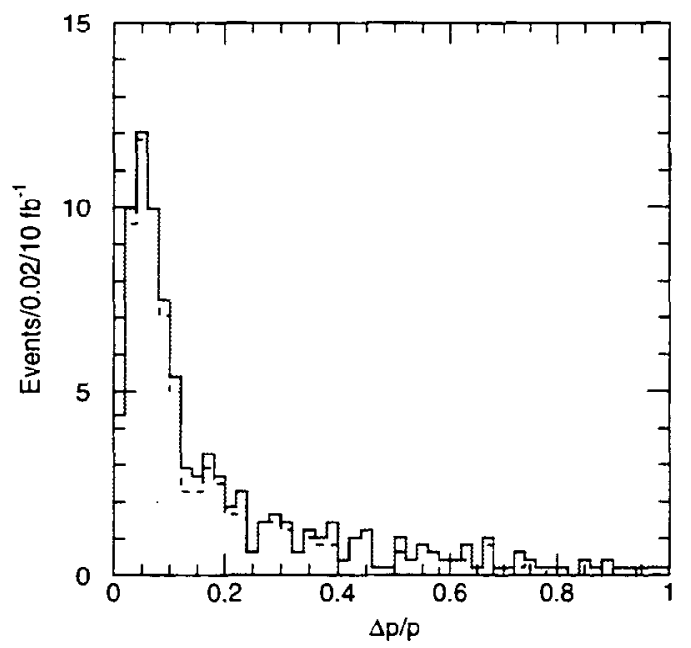

Figure 20-58 Fractional difference between reconstructed and generated gravitino momenta at Point G1a for $\chi^{2}<10$ (solid) and $\chi^{2}<1$ (dashed). lution can be selected by comparing the vector sum of their reconstructed momenta with the missing transverse momentum. Thus, this long decay chain allows SUSY events to be fully reconstructed despite the presence of two missing particles.

Events were required to have exactly four leptons and two photons satisfying the same cuts as before, with one and only one pairing into two opposite-sign, same-flavour $l^{+} l^{-} \gamma$ combinations consistent with $\tilde{\chi}_{2}^{0}$ decay. This eliminates any additional combinatorial background and gives a total of 16 solutions for the two gravitino momenta $\vec{p}_{1}$ and $\vec{p}_{2}$. These solutions can be found explicitly after straightforward algebra [20-36]. The gravitinos were assumed to give the $E_{\mathrm{T}}^{\text {miss }}$ up to detector resolution effects. Therefore, the solution that minimised

$$
\chi^{2}=\left(\frac{E_{x}^{\mathrm{miss}}-p_{1 x}-p_{2 x}}{\Delta E_{x}^{\mathrm{miss}}}\right)^{2}+\left(\frac{E_{y}^{\mathrm{miss}}-p_{1 y}-p_{2 y}}{\Delta E_{y}^{\mathrm{miss}}}\right)^{2}
$$

was selected, where the errors were calculated using assumed calorimetric missing energy resolution

$$
\Delta E_{x}^{\text {miss }}=\Delta E_{y}^{\text {miss }}=0.6 \sqrt{E_{\mathrm{T}}}+0.03 E_{\mathrm{T}}
$$


This parametrisation gives resolution values which are a factor of two to three worse than the $E_{\mathrm{T}}^{\text {miss }}$ resolution obtained from the full simulation studies described in Section 9.2.1.1. Events that satisfied $\chi^{2}<10$ were retained for further analysis.

The method was tested by comparing the reconstructed gravitino momenta with the generated momenta using the better of the two possible matches. The distribution for the fractional difference between the reconstructed and generated momenta,

$$
\frac{\Delta p}{p} \equiv \frac{\left|\vec{p}_{\text {recon }}-\vec{p}_{\text {gen }}\right|}{\left|\vec{p}_{\text {gen }}\right|},
$$

is shown in Figure 20-58 for events with $\chi^{2}<10$. The peak is at about $10 \%$, considerably larger than the typical lepton or photon resolution, and there is a substantial tail of events that presumably have missing energy from sources other than the gravitinos. The number of reconstructed events is quite small. Nevertheless, the fact that complete reconstruction with two missing particles is possible at all is interesting. A similar complete reconstruction is possible in principle for SUGRA Point 5 using the three mass constraints from decay chain $\tilde{q}_{L} \rightarrow \tilde{\chi}_{2}^{0} q \rightarrow l_{R}^{ \pm} l^{\mp} q \rightarrow \tilde{\chi}_{1}^{0} l^{+} l^{-} q$, but unfortunately the resulting errors are too large to be useful.

\subsubsection{Reconstruction of gluinos and squarks}

Events that are fully reconstructed as described in the previous section can be used to measure the squark and gluino masses using the decay chain $\tilde{q} \rightarrow \tilde{g} q \rightarrow \tilde{\chi}_{2}^{0} \bar{q} q q$. It would probably be better to study these particles using partially reconstructed combinations of jets, leptons, and photons, as was done for several of the SUGRA points rather than using the small number of fully reconstructed events. This analysis mainly serves to illustrate further the possibility of full reconstruction; it is not used in the fit to determine the parameters of the model.

Events were selected to have two $\tilde{\chi}_{2}^{0}$ 's fully reconstructed with $\chi^{2}<10$ and at least four jets with $p_{T}>75 \mathrm{GeV}$ defined using a cone $R=0.4$. Each reconstructed $\tilde{\chi}_{2}^{0}$ is then combined with two of the four hardest jets and then with a third. The resulting scatter plot of $M\left(\tilde{\chi}_{2}^{0} j j\right)$ vs $M\left(\tilde{\chi}_{2}^{0} j j j\right)$ has a broad peak near the gluino and squark masses. The two projections, each cut

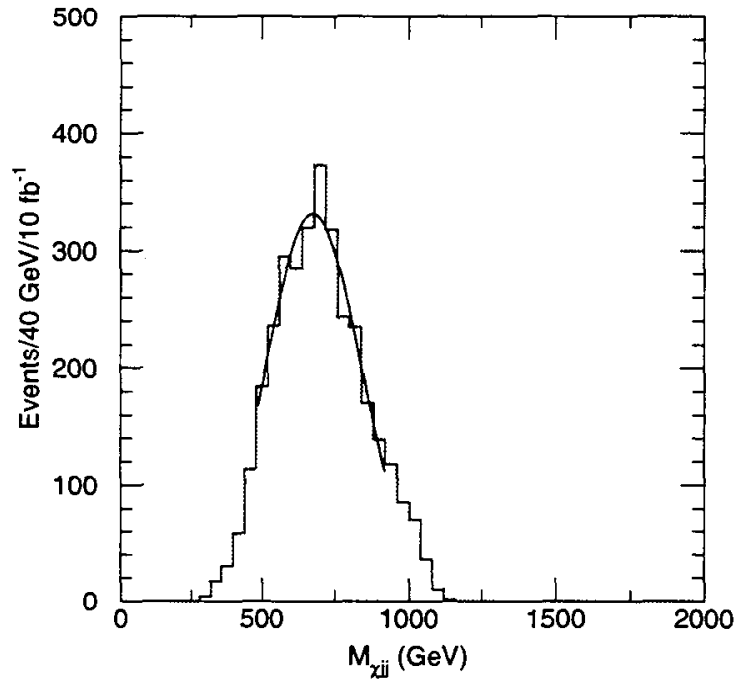

Figure 20-59 Distribution of $M\left(\tilde{\chi}_{2}^{0} j j\right)$ for $800<M\left(\tilde{\chi}_{2}^{0} j j j\right)<1000 \mathrm{GeV}$ at Point G1a.

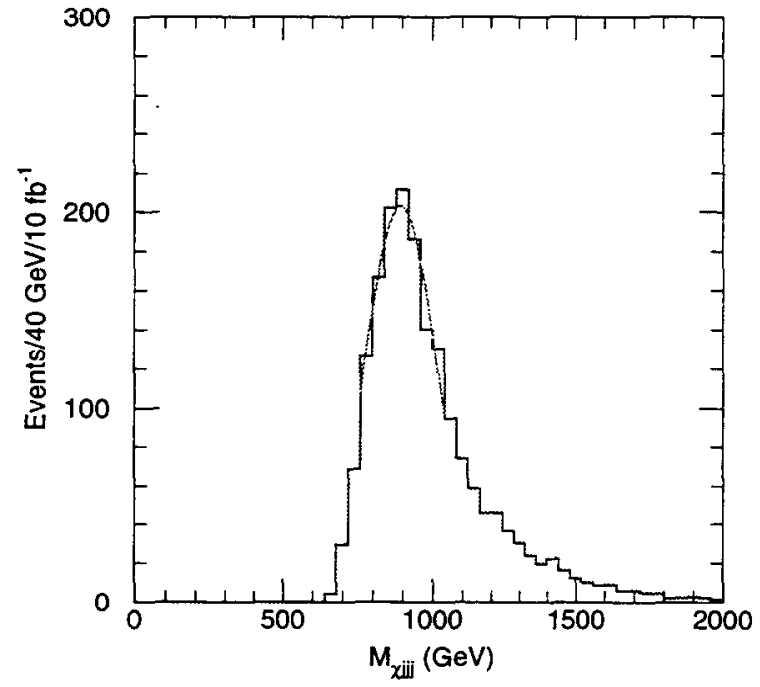

Figure 20-60 Distribution of $M\left(\tilde{\chi}_{2}^{0} j j j\right)$ for $600<M\left(\tilde{\chi}_{2}^{0} j j\right)<800 \mathrm{GeV}$ at Point G1a. 
about the peak on the other axis, are shown in Figures 20-59 and 20-60 together with Gaussian fits to guide the eye. Even with the limited number of fully reconstructed events, the statistical errors on the peaks are quite small; the errors are likely to be dominated by systematic effects such as the jet energy scale.

\subsubsection{GMSB Point G1b}

The SUSY cross section at Point G1b is $7.6 \mathrm{pb}$, the same as at Point G1a. The NLSP, the $\tilde{\chi}_{1}^{0}$, is neutral and long-lived; most of them escape the detector, giving signatures that are qualitatively like those in SUGRA models. A qualitatively new feature is that the $\tilde{\chi}_{1}^{0}$ can occasionally decay in the tracking volume, giving rise to a photon that does not point to the interaction vertex. Since the ATLAS electromagnetic calorimeter provides directional information, it can measure such photons, giving information on the $\tilde{\chi}_{1}^{0}$ lifetime and hence on $C_{\text {grav }}$. Since this is the only source of information on the global scale of SUSY breaking, it is extremely important. It will be discussed in Section 20.3.2.4 after the other signatures.

\subsubsection{Effective mass analysis}

Discovery of SUSY in inclusive distributions at this point is similar to that for SUGRA models. Events were selected to have [20-36]

- at least four jets with $p_{\mathrm{T}, 1}>100 \mathrm{GeV}$ and $p_{\mathrm{T}, 2,3,4}>50 \mathrm{GeV}$;

- $E_{T}^{\text {miss }}>\max \left(100 \mathrm{GeV}, 0.2 M_{\text {eff }}\right)$, where $M_{\text {eff }}$ is defined by Equation 20-5;

- transverse sphericity $S_{\mathrm{T}}>0.2$;

- no $\mu$ or isolated $e$ with $p_{\mathrm{T}}>20 \mathrm{GeV}$ and $|\eta|<2.5$.

With these cuts the signal exceeds the Standard Model background for $M_{\text {eff }}>1000 \mathrm{GeV}$ by about a factor of five [20-36].

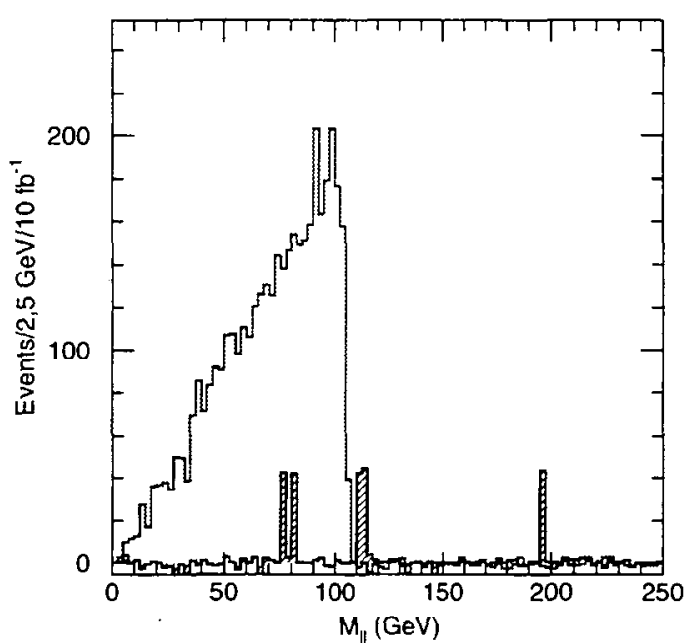

Figure 20-61 Flavour-subtracted dilepton distribution at Point G1b. The shaded histogram shows the Standard Model background; the fluctuations reflect the limited Monte Carlo statistics.

At Point G1b the decay $\tilde{\chi}_{2}^{0} \rightarrow \tilde{l}_{R}^{+} l^{\mp} \rightarrow \tilde{\chi}_{1}^{0} l^{+} l^{-}$is allowed, but the $\tilde{\chi}_{1}^{0}$ is stable rather than decaying to $\tilde{G} \gamma$. Events were selected to have $M_{\mathrm{eff}}>1000 \mathrm{GeV}, E_{\mathrm{T}}^{\mathrm{miss}}>0.1 M_{\mathrm{eff}}$, and two and only two opposite-sign leptons with $p_{\mathrm{T}}>20 \mathrm{GeV}$ and $|\eta|<2.5$. The resulting $e^{+} e^{-}+\mu^{+} \mu^{-}-e^{ \pm} \mu^{\mp}$ dilepton distribution, shown in Figure 20-61 has an endpoint at $105.1 \mathrm{GeV}$, which of course is the same as for Point G1a. There is more Standard Model background than before because there are no photons in the signal events, but the background is still small; the spikes in the background curve in the figure are fluctuations that reflect the limited Monte Carlo statistics. Hence, the error on this endpoint should again be about $0.1 \%$, limited by the absolute lepton energy scale. The sharp endpoint is characteristic of a sequential decay through a slepton. 
There is also a small peak visible in the figure from $Z$ decays which could be measured with more luminosity. This measurement would help to confirm the two body nature of the decay and potentially would provide information on the Higgsino content of the light neutralinos.

\subsubsection{Reconstruction of gluinos and squarks}

The $\tilde{\chi}_{2}^{0} \rightarrow \tilde{l}_{R}^{ \pm} l^{\mp} \rightarrow \tilde{\chi}_{1}^{0} l^{+} l^{-}$decays reconstructed in the previous section come primarily from $\tilde{g} \rightarrow \tilde{\chi}_{2}^{0} q \bar{q}$, where the gluino may be produced directly or from squark decay. To attempt to extract these signals, events were selected as before with the additional requirement of two hard jets with $p_{\mathrm{T}}>125 \mathrm{GeV}$. The $e^{+} e^{-}+\mu^{+} \mu^{-}-e^{ \pm} \mu^{\mp}$ dilepton pair was then combined with any two jets having $p_{\mathrm{T}}>25 \mathrm{GeV}$. The resulting $l^{+} l^{-} j j$ mass distribution, Figure 20-62, has a broad peak but no clear structure. It is, however, sensitive to the gluino mass: the same figure also shows as a dotted curve the distribution for a sample with the gluino mass increased from $747 \mathrm{GeV}$ to $800 \mathrm{GeV}$. These could probably be distinguished statistically at high luminosity; the systematic errors need further study.

The branching ratio for $\tilde{g} \rightarrow \tilde{\chi}_{2}^{0} b \bar{b}$ is $6.5 \%$. The $l^{+} l^{-} j j$ distribution using jets tagged as $b$ 's is shown in Figure 20-63. This has a structure that reflects the kinematic endpoint of this decay (for massless jets) at

$$
\left[\left(M_{\bar{g}}-M_{\tilde{\chi}_{2}^{0}}\right)^{2}+M_{l l}^{2}+\frac{M_{\tilde{g}}-M_{\tilde{\chi}_{2}^{0}}}{2 M_{\tilde{\chi}_{2}^{0}}}\left(M_{\tilde{\chi}_{2}^{0}}^{2}-M_{l}^{2}+\frac{\left(M_{\tilde{\chi}_{2}^{0}}^{2}+M_{l}^{2}\right)\left(M_{\tilde{l}}^{2}-M_{\bar{\chi}_{1}^{0}}^{2}\right)}{2 M_{l}^{2}}\left(1+\frac{M_{\tilde{\chi}_{2}^{0}}^{2}-M_{l}^{2}}{2 M_{\tilde{\chi}_{2}^{0}}^{2} M_{\bar{l}}}\right)\right)\right]^{1 / 2},
$$

where $M_{l l}$ is the value of the dilepton edge. For Point G1b this $l^{+} l^{-} j j$ endpoint is at $629 \mathrm{GeV}$, while for a gluino mass of $800 \mathrm{GeV}$, it becomes $673 \mathrm{GeV}$. These values are approximately consistent with the structure of the solid and dashed curves in Figure 20-63. While the event rates are low, these curves could be distinguished statistically with higher luminosity; however, the impact of pileup on the analysis has not been studied.

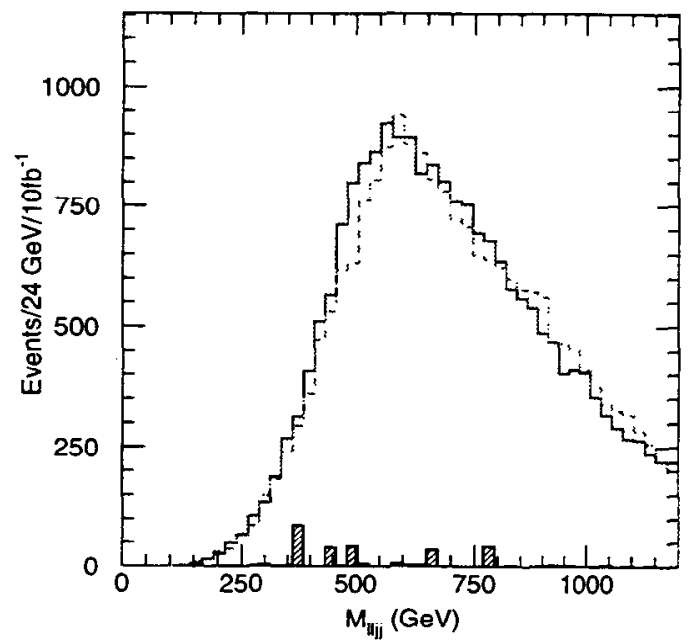

Figure 20-62 $l^{+} l^{-} j j$ invariant mass distribution for Point G1b (solid curve) and for the gluino mass shifted to $800 \mathrm{GeV}$ (dashed curve).

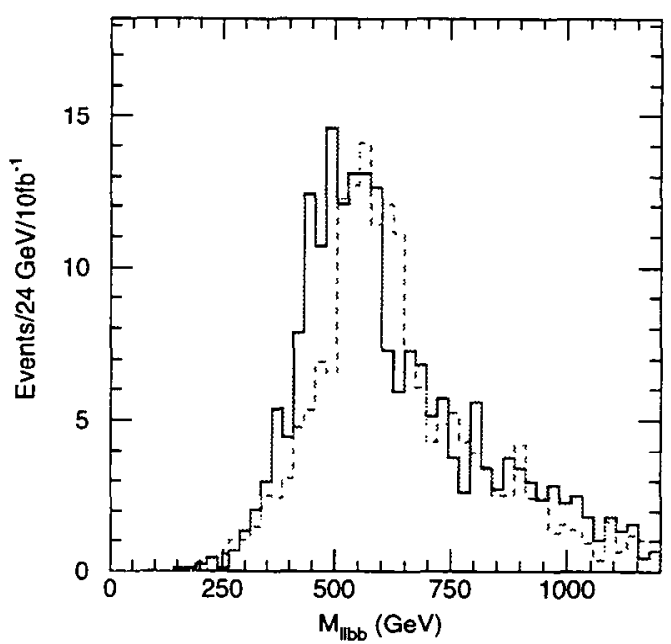

Figure 20-63 Same as Figure 20-62 for $b$-jets. The dashed curve is for the sample with $M_{\tilde{g}}=800 \mathrm{GeV}$. No Standard Model event passed the cuts. 
The endpoint of the dijet mass distribution should be sensitive to $M_{\bar{g}}-M_{\bar{\chi}^{0}}$, but the endpoint is not at all sharp due to the large combinatorial background. It is also possible to add another jet, thereby getting a handle on the squark mass. Again the rates are low, and while there is a difference in shape, there is not a clear kinematic endpoint. Since these analyses do not illustrate any new techniques, they will not be shown here, although they are used in the fitting described in Section 20.3.5.

\subsubsection{Measurement of $\tilde{\chi}_{1}^{0}$ lifetime}

If SUSY breaking occurs only in the messenger sector, then the $\tilde{\chi}_{1}^{0}$ lifetime is short, $c \tau \approx 1.1 \mathrm{~mm}$. It is possible, however, that SUSY breaking in the messenger sector is induced by higher order effects in some new interaction, giving a much longer lifetime. The parameter $C_{\text {grav }}=1000$ assumed for Point G1b corresponds to $c \tau=1.1 \mathrm{~km}$. Even though this decay length is large compared to the size of the tracking volume, it can still be measured by counting the number of nonpointing photons from the small number of $\tilde{\chi}_{1}^{0} \rightarrow \tilde{G} \gamma$ that occur inside the detector.

The ATLAS electromagnetic calorimeter is particularly well suited for this because it has narrow strips in the first compartment that give good resolution in $\theta$. As described in Section 4.4.2.2, the resolution for photons in the barrel that are close to pointing is

$$
\Delta \theta=\frac{60 \mathrm{mr}}{\sqrt{E(\mathrm{GeV})}}
$$

The resolution for non-pointing photons has been studied [20-37] for single photons from about $7500 \tilde{\chi}_{1}^{0} \rightarrow \tilde{G} \gamma$ decays and also for samples of $50 \mathrm{GeV}$ photons generated at fixed angles. Two algorithms have been developed, which can reconstruct photons with reasonable accuracy for all values of $\Delta \theta$. These are described in Section 4.4.2.2.

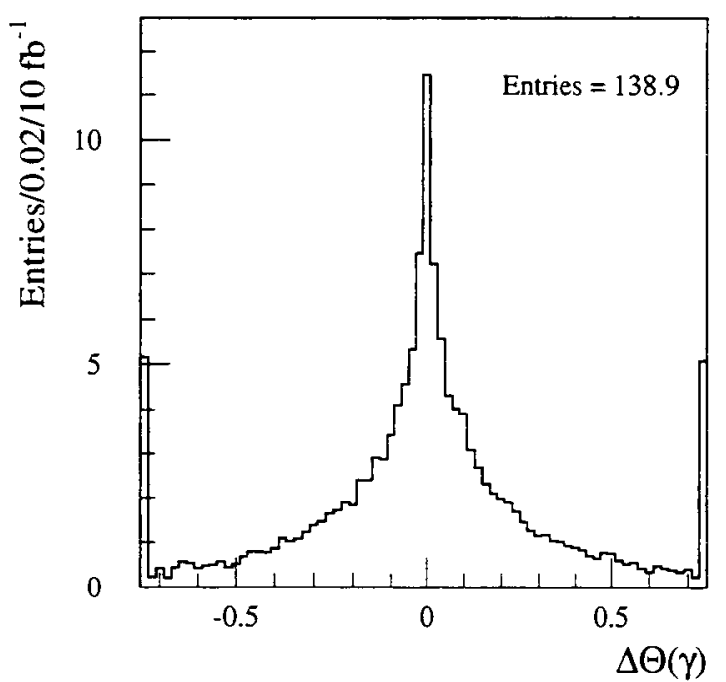

Figure 20-64 Non-pointing angle $\Delta \theta$ for photons in the barrel calorimeter from $\tilde{\chi}_{1}^{0} \rightarrow \tilde{G} \gamma$ at Point $G 1 b$ for the assumed lifetime $c \tau=1.1 \mathrm{~km}$. The first and last bins contain the overflows

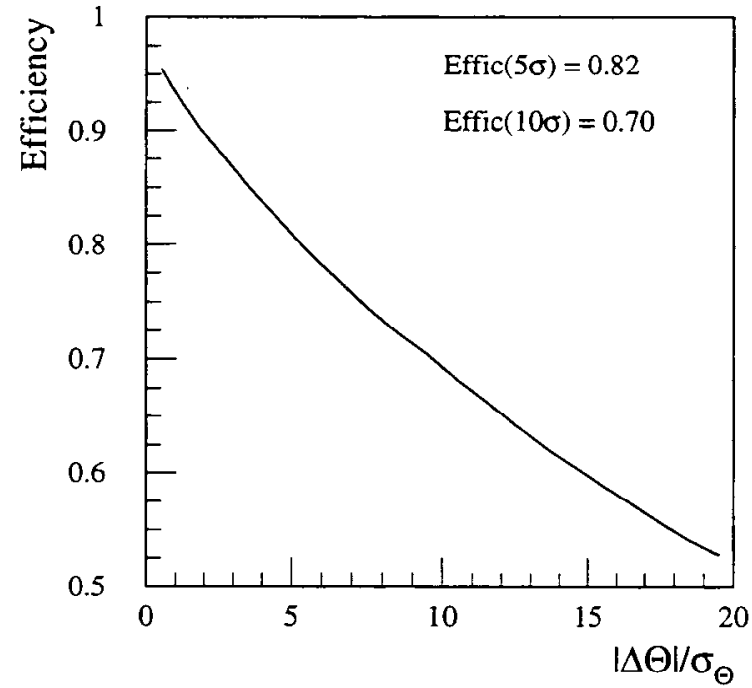

Figure 20-65 Efficiency vs significance for a photon from $\tilde{\chi}_{1}^{0} \rightarrow \tilde{G} \gamma$ to be non-pointing at Point G1b. 
For this analysis [20-37] it is assumed that the lifetime $c \tau$ is large compared to the length $L(\eta)$ inside of the tracker, so that the decay probability is given by $L(\eta) /(\beta \gamma c \tau)$ and the decays occur uniformly along the path length. For Point G1b a total of $152000 \tilde{\chi}_{1}^{0}$ are produced corresponding to an integrated luminosity of $10 \mathrm{fb}^{-1}$. For the assumed $c \tau=1.1 \mathrm{~km}, 180$ of these will decay inside the tracking volume. The mean energy of these photons is $84.4 \mathrm{GeV}$. An analysis based on ATLFAST found that $77.3 \%$ of the photons hit the barrel calorimeter, and $82.5 \%$ of these have $p_{\mathrm{T}}>20 \mathrm{GeV}$ and are isolated.

The angular distribution of these photons relative to the nominal vertex direction, $\Delta \theta$, is shown in Figure 20-64 including the effect of the angular resolution. While the distribution peaks at zero, the angles are generally large compared to the resolution. This is reflected in the efficiency to detect an isolated photon as non-pointing as a function of the significance, shown in Figure 2065. Requiring that $\Delta \theta$ be non-zero by $5 \sigma$ gives an efficiency of $82 \%$, or a total of 94 detected non-pointing photons, for an overall efficiency of $52 \%$. Converting this rate to a measurement of a lifetime requires that the mass and momentum distribution of the $\tilde{\chi}_{1}^{0}$ be determined from other measurements. The rate for prompt photons in SUSY events has not been calculated but is expected to be of order $\alpha / \pi$, so the background should be much less than one event.

If no non-pointing photons are detected for an integrated luminosity of $30 \mathrm{fb}^{-1}$, then the $95 \%$ confidence level lower limit on $\tilde{\chi}_{1}^{0} \rightarrow \tilde{G} \gamma$ would be about $c \tau=100 \mathrm{~km}$, a factor of $10^{8}$ longer than the value for $C_{\text {grav }}=1$. Since

$\Gamma(\tilde{X} \rightarrow \tilde{G} X)=\frac{1}{16 \pi} \frac{M_{\tilde{X}}^{5}}{F^{2}}\left(1-\frac{M_{X}^{2}}{M_{\tilde{X}}^{2}}\right)^{4}$,

this corresponds to a value of $F$ that is $10^{4}$ times larger than that in the messenger sector. Of course this assumes that the $\Delta \theta$ resolution is really Gaussian. So far it has been studied only for single photons, not for complete events.

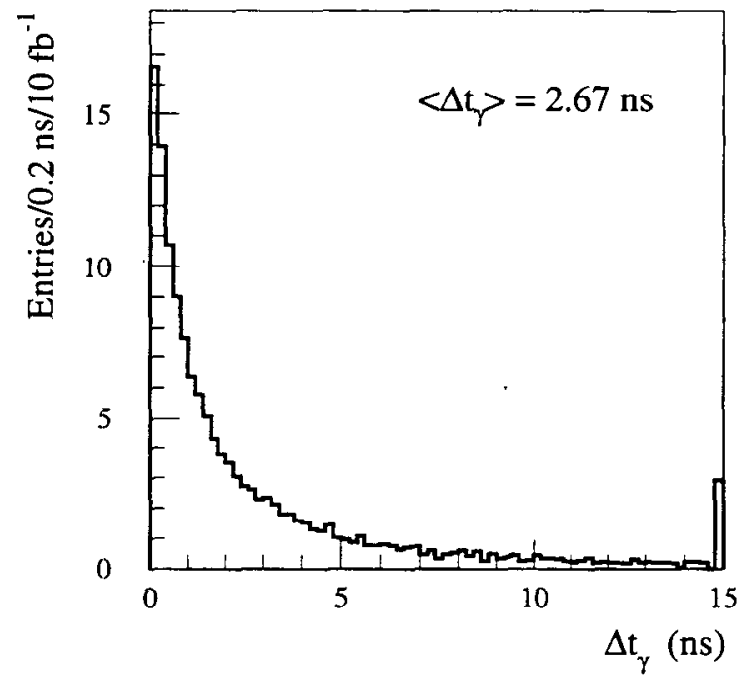

The photons from $\bar{\chi}_{1}^{0} \rightarrow \tilde{G} \gamma$ will in general be delayed relative to prompt photons as shown in Figure 20-66. Both the velocity of the $\tilde{\chi}_{1}^{0}$ and the geometry of the path contribute to the

Figure 20-66 Time delay distribution in the EM calorimeter of photons from $\tilde{\chi}_{1}^{0} \rightarrow \tilde{G} \gamma$ at Point G1b. The last bin contains the overflow.

delay. The mean delay, $2.67 \mathrm{~ns}$, is long compared to the time resolution of about $100 \mathrm{ps}$ for the ATLAS electromagnetic calorimeter. This provides an independent way to detect non-prompt photons and a cross-check on the whole analysis.

\subsubsection{GMSB Point G2a}

At Point G2a the NLSP is a charged slepton, the $\tilde{\tau}_{1}$. The splitting between the NLSP and the right-handed sleptons $\tilde{l}_{R}$ of the first two generations is small as is typical in GMSB models if $\tan \beta$ is not too large, so the decays $\tilde{l}_{R}^{ \pm} \rightarrow \tilde{\tau}_{1} \tau l^{ \pm}$are not kinematically allowed. The decays $\tilde{l}_{R} \rightarrow \tilde{\tau}_{1} v_{\tau} v_{l}$ occur only through left-right mixing terms proportional to the lepton mass and are small even compared to gravitino decays. Hence the $\tilde{e}_{R}, \bar{\mu}_{R}$ and $\tilde{\tau}_{1}$ are co-NLSP's that all decay directly to gravitinos with $c \tau=52 \mu \mathrm{m}$. The SUSY production cross section is $23 \mathrm{pb}$, larger than 
for Points G1a/b because the squarks are lighter, and all of these events decay through the sleptons to electrons, muons or $\tau$ 's. Discovery of such a signal is obviously trivial, and there are many signatures to be studied.

\subsubsection{Dilepton distributions}

Neutralinos can decay via $\tilde{\chi}_{i}^{0} \rightarrow \tilde{l}^{ \pm} l^{\mp} \rightarrow \tilde{G} l^{+} l^{-}$with substantial branching ratios to give oppositesign, same-flavour dileptons. Since the neutralinos come mainly from the decay of squarks and gluinos, events were selected to have [20-36]

- at least four jets with $p_{\mathrm{T}}>25 \mathrm{GeV},|\eta|<2.5$, and having at least four tracks each with $p_{\mathrm{T}}>1 \mathrm{GeV}$;

- $M_{\text {eff }}>400 \mathrm{GeV}$;

- $E_{\mathrm{T}}^{m i s s}>0.2 M_{\mathrm{eff}}$;

- Two opposite-charge leptons with $p_{\mathrm{T}}>10 \mathrm{GeV}$ and $|\eta|<2.5$.

The jet multiplicity cut is intended to remove $\tau$ 's from the jet sample. The flavour-subtracted $e^{+} e^{-}+\mu^{+} \mu^{-}-e^{ \pm} \mu^{\mp}$ dilepton mass was then formed to cancel backgrounds from two independent leptonic decays of charginos or from Standard Model processes. This distribution is shown in Figures 20-67 and 20-68 on two different scales. There are two separate edges, one from $\tilde{\chi}_{1}^{0}$ decay ( $31 \%$ branching ratio per flavour) at

$$
\sqrt{M_{\tilde{\chi}_{1}^{0}}^{2}-M_{\tilde{l}_{R}}^{2}}=52.1 \mathrm{GeV}
$$

and a second from $\tilde{\chi}_{2}^{0}$ decay ( $23 \%$ branching ratio per flavour) at

$$
\sqrt{M_{\bar{\chi}_{2}^{0}}^{2}-M_{\tilde{l}_{R}}^{2}}=175.9 \mathrm{GeV}
$$

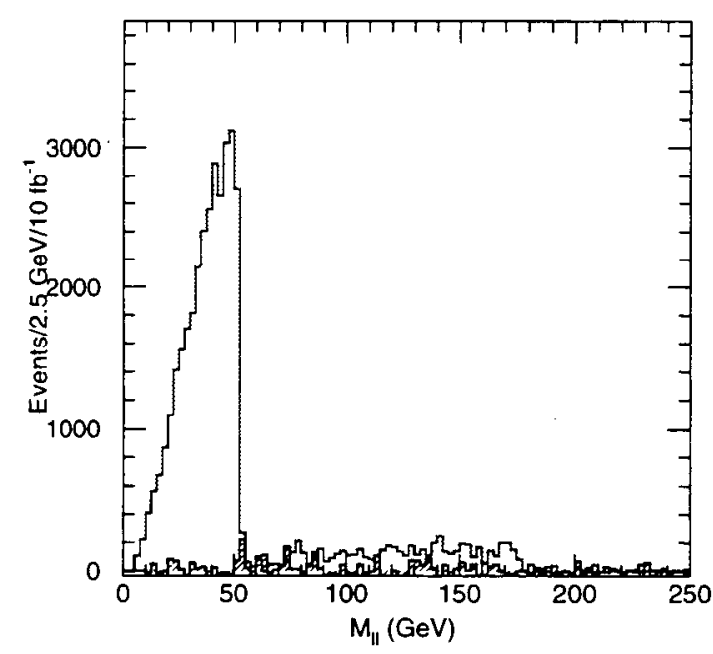

Figure 20-67 $e^{+} e^{-}+\mu^{+} \mu^{-}-e^{ \pm} \mu^{\mp}$ dilepton distribution at Point G2a. The fluctuations in the Standard Model background (shaded) are due to Monte Carlo statistics; the true background is small.

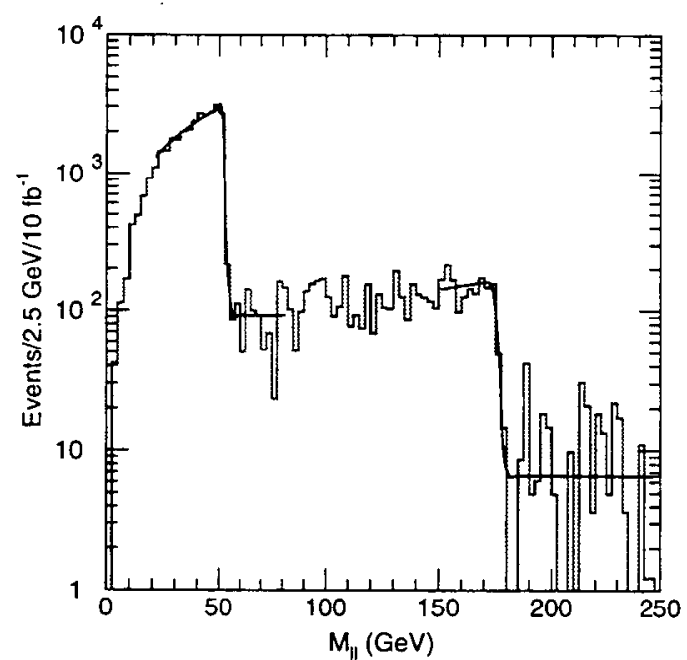

Figure 20-68 Same as Figure 20-67 on a logarithmic scale. Fits using the expected form, Equation 20-6, smeared with a Gaussian are also shown. 
While the Standard Model background appears to be significant for the second edge, this is an artifact of the limited Monte Carlo statistics. In the region $60-170 \mathrm{GeV}$ the background can be estimated to be $0 \pm 60$ events, compared to a total signal of about 4000 events. The statistics in these plots corresponds to about $10 \mathrm{fb}^{-1}$. A fit using the expected form of the distribution, Equation 20-6, smeared with a Gaussian gave

$$
\begin{aligned}
M=52.266_{-0.045}^{+0.058} \mathrm{GeV} & \sigma & =0.86 \mathrm{GeV} \\
M=175.46_{-0.22}^{+0.21} \mathrm{GeV} & \sigma & =6.5 \mathrm{GeV}
\end{aligned}
$$

where the errors are determined using MINOS. Thus the errors on the upper edge are statistics limited even for design luminosity. Including systematic effects, the errors are estimated to be 0.07 and $0.27 \mathrm{GeV}$ for $10 \mathrm{fb}^{-1}$ and 0.05 and $0.18 \mathrm{GeV}$ for $100 \mathrm{fb}^{-1}$. The upper edge seems wider than expected from detector resolution.

\subsubsection{Detection of $\tilde{q}_{R} \rightarrow \tilde{\chi}_{1}^{0} q$}

Right-handed squarks are copiously produced both directly and through gluino decay, and they can decay via $\tilde{q}_{R} \rightarrow \tilde{\chi}_{1}^{0} q \rightarrow \tilde{l}^{ \pm} l^{\mp} q \rightarrow \tilde{G} l^{+} l^{-} q$. To select this mode, a dilepton pair with high $p_{\mathrm{T}}$ below the $\tilde{\chi}_{1}^{0}$ edge and two jets were required in addition to the cuts in the previous subsubsection:

- $M_{l l}<52.2 \mathrm{GeV}$ and $p_{\mathrm{T}, l l}>75 \mathrm{GeV}$;

- two jets with $p_{\mathrm{T}}>50 \mathrm{GeV},|\eta|<2.5$, and at least four charged tracks each with $p_{\mathrm{T}}>1 \mathrm{GeV}$.

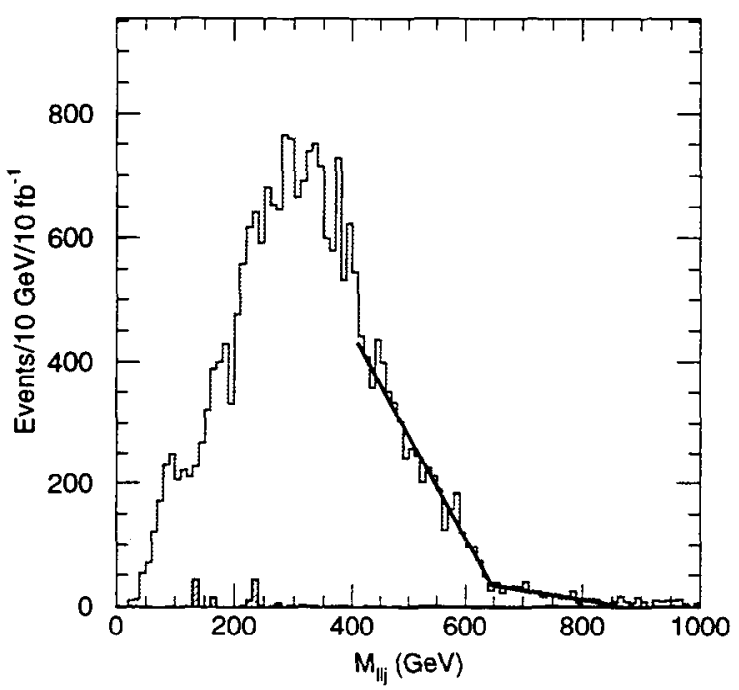

Figure 20-69 Distribution of the smaller $l l j$ mass for events with two leptons and four jets at Point G2a. The dashed line is a linear fit over $390-590 \mathrm{GeV}$. The Standard Model background is the hatched histogram.

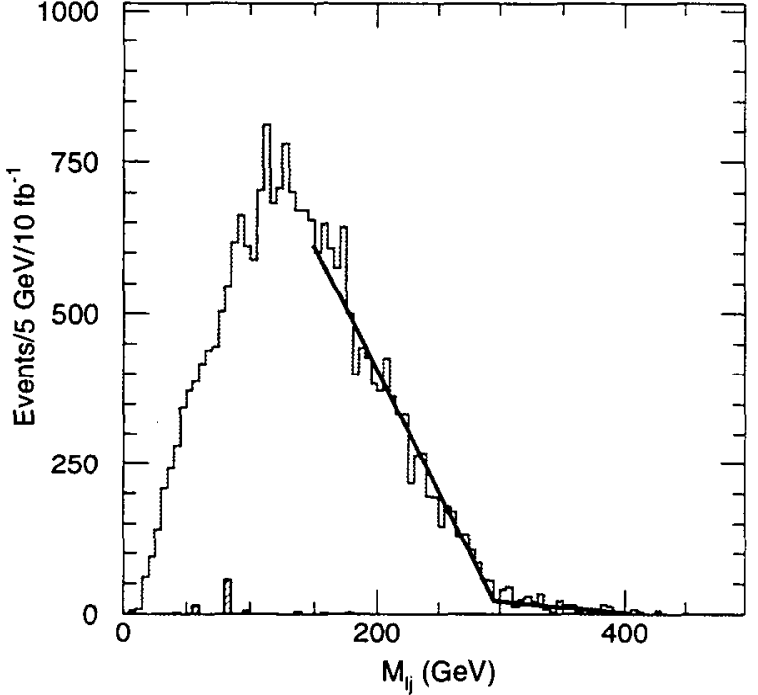

Figure 20-70 lj mass distribution for the same sample as in Figure 20-69. The dashed lines are linear fits over $150-280 \mathrm{GeV}$ and $305-400 \mathrm{GeV}$. 
Again the $e^{+} e^{-}+\mu^{+} \mu^{-}-e^{ \pm} \mu^{\mp}$ combination is used to eliminate backgrounds. The dilepton pair was then combined with each of the two hardest jets in the event. Since one of these two should come from the squark decay, the combination with the smaller mass was selected as usual. Then the $l l q$ mass distribution, Figure 20-69, has a linear vanishing at the expected endpoint

$$
M_{l l q}^{\max }=\sqrt{M_{\tilde{q}_{R}}^{2}-M_{l_{R}}^{2}}=640.1 \mathrm{GeV}
$$

while the $l q$ mass distribution, Figure 20-70, shows the expected endpoint at

$$
M_{l q}^{\max }=\sqrt{M_{\bar{q}_{R}}^{2}-M_{\bar{\chi}_{1}^{0}}^{2}} \sqrt{1-\frac{M_{\hat{l}_{R}}^{2}}{M_{\tilde{\chi}_{1}^{0}}^{2}}}=289.1 \mathrm{GeV} .
$$

The $l l, l l j$, and $l j$ distributions from this three-step decay chain provide three measured endpoints from which the three masses involved can be determined assuming only that the gravitino mass is negligible:

$$
\begin{aligned}
M_{\tilde{l}_{R}} & =\frac{M_{l l} \sqrt{M_{l l q}^{2}-M_{l q}^{2}-M_{l l}^{2}}}{M_{l q}} \\
M_{\tilde{\chi}_{1}^{0}} & =\sqrt{M_{l l}^{2}+M_{\tilde{l}_{R}}^{2}} \\
M_{\tilde{q}_{R}} & =\sqrt{M_{l l q}^{2}+M_{l_{R}}^{2}}
\end{aligned}
$$

The determination of these masses depends only on the existence of the decay chain and does not assume the minimal GMSB or any other model, again reflecting the power of utilising multi-step decay chains. Of course the interpretation of the masses as those of the $\tilde{q}_{R}, \tilde{\chi}_{1}^{0}$, and $\tilde{l}_{R}$ is model dependent.

\subsubsection{Detection of $\tilde{\chi}_{1}^{ \pm}$}

About $50 \%$ of the $\tilde{q}_{L}$ decays at this point occur through the $\tilde{\chi}_{1}^{ \pm}$. While in general charginos are hard to reconstruct, the decay chain

$\tilde{\chi}_{1}^{+} \rightarrow \tilde{v} l^{+} \rightarrow \tilde{\chi}_{1}^{0} v l^{+} \rightarrow \tilde{l}_{R}^{+} l^{\mp} v l^{+} \rightarrow \tilde{G} l^{+} l^{-} v l^{+}$

has a combined branching ratio of $29 \%$ and gives a very nice trilepton signature. Events were selected to have at least three isolated leptons plus the same jets and other cuts described before. One pair of leptons was required to form an opposite-sign, same-flavour pair with $40<M_{l l}<52 \mathrm{GeV}$, so that it is likely to have come from a $\tilde{\chi}_{1}^{0}$ decay. It was also required that there be no other opposite-sign, same-flavour pair with $M_{l l}<175 \mathrm{GeV}$, the

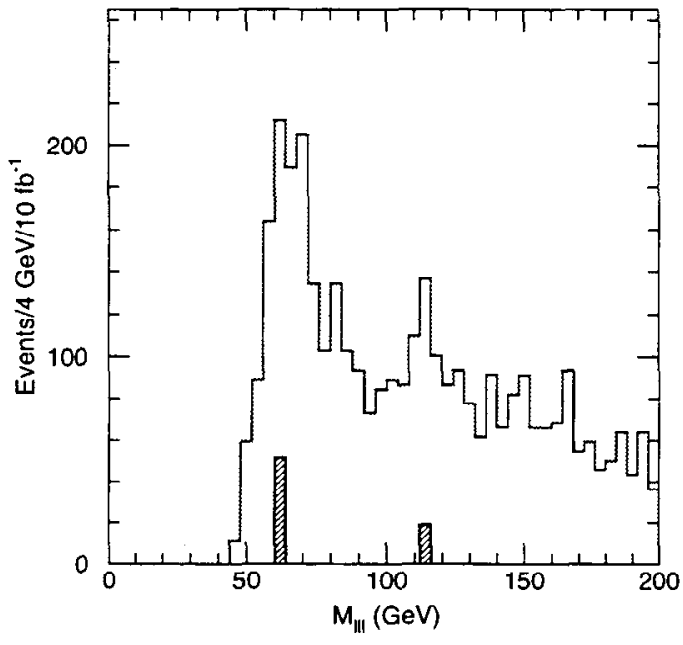

Figure 20-71 Trilepton mass distribution for chargino signal at Point G2a. The shaded histogram is the Standard Model background. 
endpoint for $\tilde{\chi}_{2}^{0}$ decay. The $l l l$ mass distribution after these cuts is shown in Figure 20-71. If the three leptons come from the above chargino decay chain, then the distribution should vanish linearly at the kinematic endpoint

$$
\sqrt{M_{\tilde{\chi}_{1}^{+}}^{2}-M_{\tilde{v}}^{2}+M_{\tilde{\chi}_{1}^{0}}^{2}-M_{l_{R}}^{2}}=85.75 \mathrm{GeV}
$$

There is a lot of background, but nevertheless there is evidence for structure in the distribution at this point. While it seems hard to extract a precision measurement because of the background, this distribution does provide evidence for the existence of the chargino and a consistency check on the model.

\subsubsection{GMSB Point G2b}

At this point the NLSP is the $\tilde{\tau}_{1}$, which has $c \tau \approx 1 \mathrm{~km}$ and so (almost) always decays outside the detector. Since the decay $\tilde{l}_{R} \rightarrow \tilde{\tau}_{1} \tau l$ is not kinematically allowed, the $\tilde{e}_{R}$ and $\tilde{\mu}_{R}$ also are long-lived, decaying to gravitinos with about the same lifetime. Each event therefore contains two quasi-stable heavy particles which pass through the calorimeter and look essentially like muons in the detector except that they have $\beta<1$, as shown in Figure 20-72. The slepton masses can be measured using the ATLAS muon system as a time-of-flight system, and the SUSY events can then be fully reconstructed [20-36]. It is again important to search for slepton decays in order to determine the true SUSY breaking scale. It should be possible to see such decays in the central tracker, but this is a difficult pattern recognition problem which has not yet been addressed. It would also be possible to detect $\tilde{e} \rightarrow \tilde{G} e$ using the same method as in

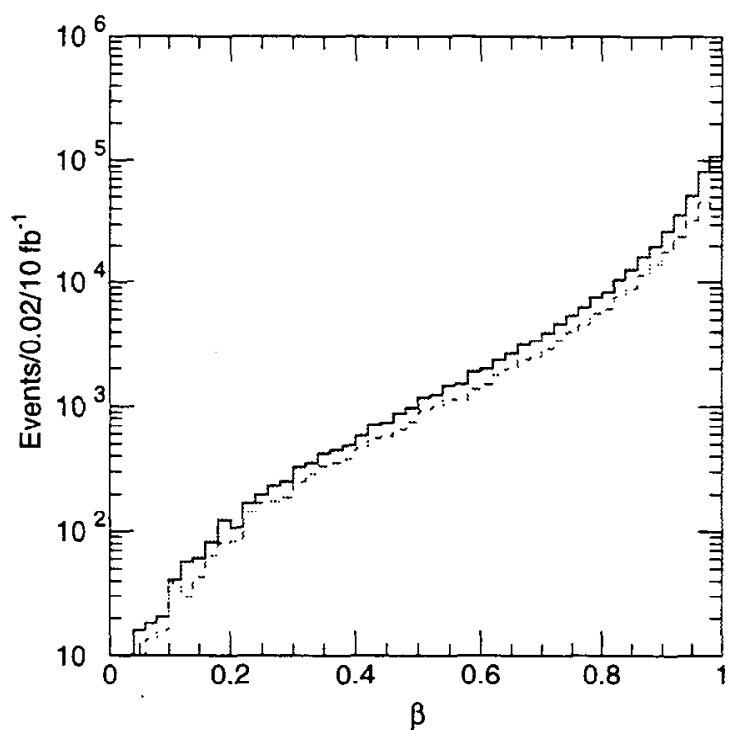

Figure 20-72 Generated slepton velocity distribution at Point G2b. The dashed curve shows the distribution for $\eta<1$. Section 20.3.2.4. The NLSP could also be separated from the SM particles exploiting the $d E / d x$ measurement in the TRT detector, using the algorithm described in Section 3.4.4 for charged hadrons. A full study taking into account the time delay of the NLSP in the TRT straws still remains to be performed.

\subsubsection{Trigger}

The events at this point can be triggered using either the sleptons or the calorimeter information. The velocity distribution of the produced sleptons, shown in Figure 20-72, has a mean value of about 0.9 . This implies that on average the sleptons reach the muon trigger chambers several nanoseconds late but still within the trigger acceptance [20-38]. One must of course be sure to record the hits from even very slow sleptons since these are the most useful for the timeof-flight measurement. 


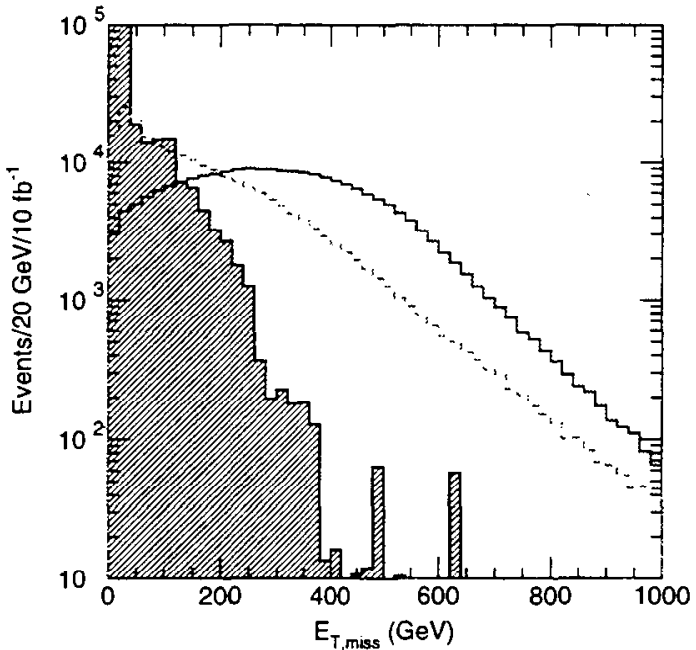

Figure 20-73 Calorimetric $E_{\mathrm{T}}^{\text {miss }}$ distribution at Point G2b. The dashed curve shows the true $E_{T}^{\text {miss }}$ including sleptons, and the shaded histogram shows the Standard Model dimuon background.

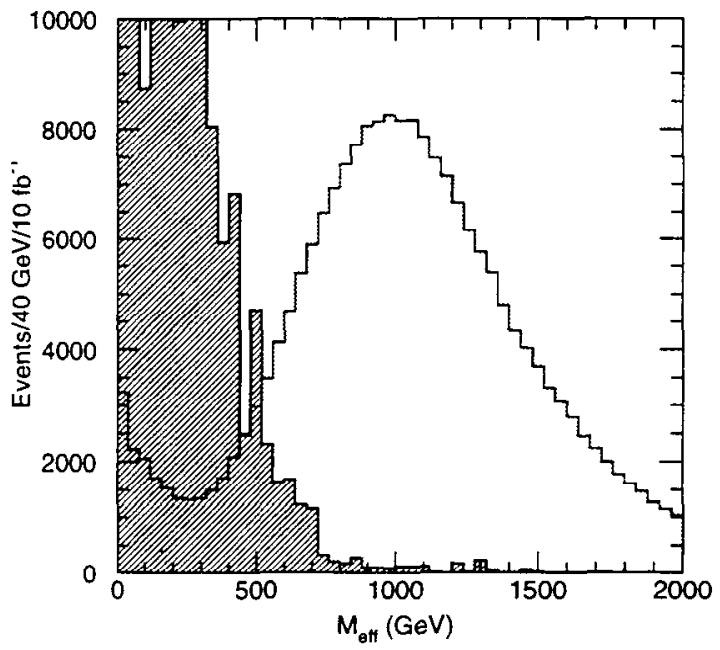

Figure 20-74 Effective mass distribution not including sleptons at Point G2b. The shaded histogram shows the Standard Model dimuon background.

The $E_{\mathrm{T}}^{\text {miss }}$ as measured by the calorimeter, Figure 20-73, is large since the sleptons lose energy only by $d E / d x$. The $E_{\mathrm{T}}^{\text {miss }}$ including the sleptons is much smaller, coming only from neutrinos produced in the cascade decays and from resolution effects. The typical effective mass, Figure 20-74, is also large, with a mean value of about $1000 \mathrm{GeV}$ characteristic of gluino and squark production. However, there is also a peak at $M_{\mathrm{eff}}=0$ from the direct production of sleptons and gauginos; it is necessary to rely on the muon trigger for these events.

\subsubsection{Slepton mass determination}

The mass of the sleptons can be measured using the muon system as a time-of-flight system: the precision chambers and trigger chambers together provide a time resolution of about 1 ns [20-38]. This initial estimate has now been verified and refined [20-39] using DICE to fully simulate the response of the MDT chambers to slow tracks and ATRECON and MUONBOX to reconstruct them. An effective time resolution of $0.65 \mathrm{~ns}$ was obtained in this study (see Section 6.4). The momentum resolution for the $\tilde{\tau}_{1}$ at momenta $p \leq M$ is somewhat worse than the one for muons which was assumed in this analysis because of multiple scattering. This difference, however, does not affect the conclusions on $\tilde{\tau}_{1}$ mass measurement.

For each slepton with $|\eta|<2.5$ the time delay

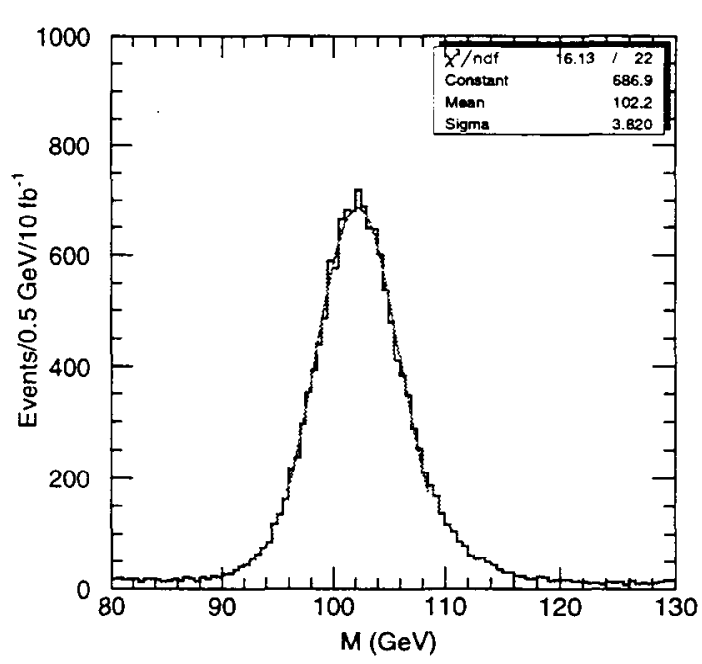

Figure 20-75 Reconstructed slepton masses at Point $\mathrm{G} 2 \mathrm{~b}$ for a $1 \mathrm{~ns}$ time resolution and $\Delta t>10 \mathrm{~ns}$.

relative to a particle with $\beta=1$ to reach the outside of the muon system, taken to be a cyl- 
inder with a radius of $10 \mathrm{~m}$ and a half-length of $20 \mathrm{~m}$, was calculated using the generated momentum and was then smeared with a $1 \mathrm{~ns}$ Gaussian resolution. The resulting time delay $\Delta t$ and measured momentum $p$ were then used to calculate the mass. The resulting mass distribution is shown in Figure 20-75 for sleptons having $10<\Delta t<50 \mathrm{~ns}$. The fitted mean value agrees very well with the average, $102.2 \mathrm{GeV}$, for the generated mixture of sleptons. The width of the distribution results from a combination of the time and momentum resolutions; even for very large $\Delta t$ it is not possible to resolve the $\tilde{\tau}_{1}$ and $\tilde{l}_{R}$ masses, 101.35 and $102.67 \mathrm{GeV}$. Because of this the upper limit on $\Delta t$ is not critical; there would be little loss if it were restricted to one bunch crossing.

\subsubsection{Reconstruction of $\tilde{\chi}_{1}^{0}, \tilde{\chi}_{2}^{0}$, and $\tilde{\chi}_{4}^{0}$}

Since the $\tilde{l}_{R}$ are quasi-stable, the decays $\tilde{\chi}_{i}^{0} \rightarrow \tilde{l}_{R} l$ can be fully reconstructed. Events were selected to have at least three electrons, muons, or quasi-stable sleptons with $|\eta|<2.5$ and $p_{\mathrm{T}}>10 \mathrm{GeV}$. The two highest $p_{\mathrm{T}}$ particles among the sleptons and muons were assumed to be sleptons with the average slepton mass measured in the previous section; the rest were considered as muons. No time-of-flight cuts were made because the Standard Model background is already negligible. The sleptons were then combined with electrons or muons. The mass distribution for all $\bar{l}_{R}^{ \pm} l^{\mp}$ combinations is shown in Figure 20-76. There are two clear peaks at the $\tilde{\chi}_{1}^{0}$ and $\tilde{\chi}_{2}^{0}$ masses and a small peak at the $\tilde{\chi}_{4}^{0}$ mass. The rather strange shape of the $\tilde{\chi}_{1}^{0}$ peak results from the fact that the splitting between the $\tilde{\chi}_{1}^{0}$ and the $\tilde{l}_{R}$ is small, so the mass is dominated by the slepton rest mass.

The determination of the slepton masses can at this point be refined, since for events in the gaugino peaks the flavour of the slepton is tagged by that of the lepton. Events were selected within $\pm 5 \mathrm{GeV}$ of the $\tilde{\chi}_{1}^{0}$ peak, and the time-of-flight analysis in Section 20.3.4.2 was repeated. The resulting mass distribution, Figure 20-77, has a mean of $102.8 \mathrm{GeV}$, quite close to the $102.67 \mathrm{GeV}$ mass of the $\tilde{e}_{R}$ and $\tilde{\mu}_{R}$, which are expected to be degenerate in GMSB models. The

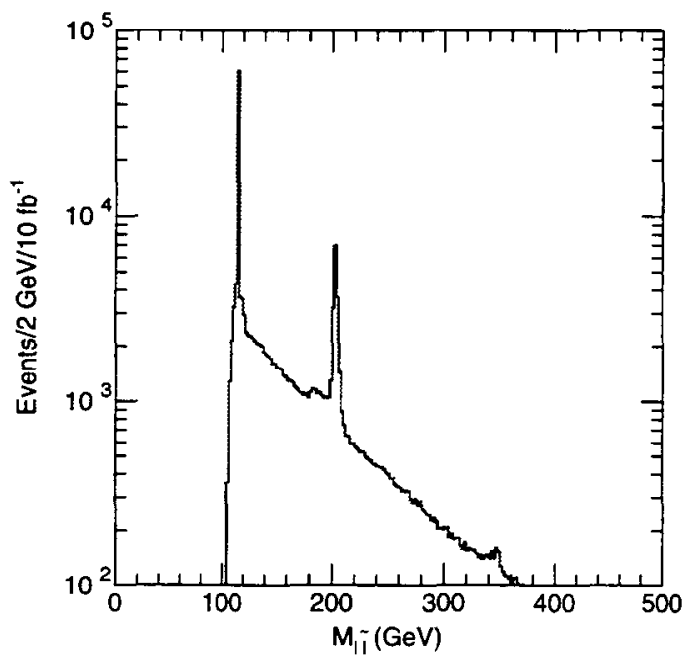

Figure 20-76 $\tilde{l}_{R}^{ \pm} l^{\mp}$ mass distribution for Point G2b.

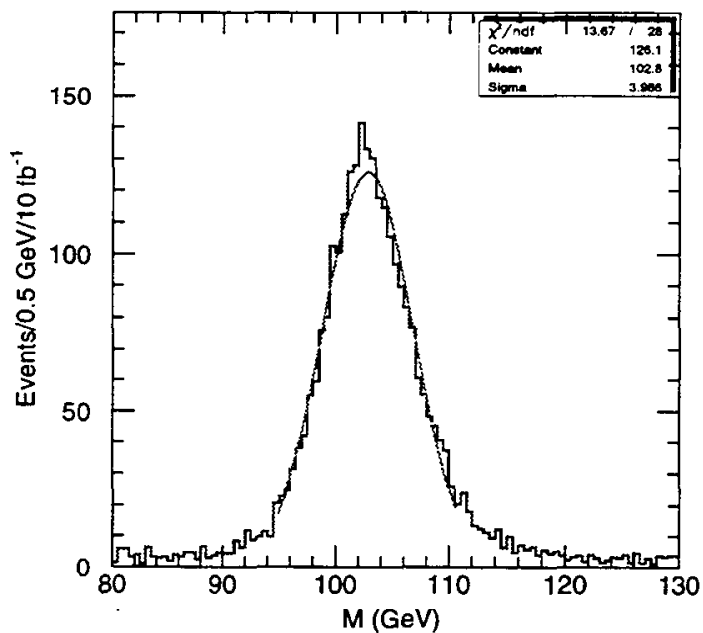

Figure 20-77 Same as Figure 20-75 for events within a $\pm 5 \mathrm{GeV}$ window of the $\tilde{\chi}_{1}^{0}$ peak in Figure 20-76. 
statistical error is about $\pm 0.1 \mathrm{GeV}$ for $10 \mathrm{fb}^{-1}$. The systematic error is expected to be at the $0.1 \%$ level. Thus it is possible to distinguish these masses from the average slepton mass measured previously, thereby constraining the $\tilde{\tau}_{1}$ mass as well.

\subsubsection{Extraction of $\tilde{l}_{L}$ and $\tilde{v}$}

Left-handed sleptons $\tilde{l}_{L}$ are produced mainly from decays of heavy gauginos although there is also some direct Drell-Yan production. They can decay via $\tilde{l}_{L} \rightarrow \tilde{\chi}_{1}^{0} l \rightarrow \tilde{l}_{R}^{ \pm} l^{\mp} l$. To reconstruct this process, events within $\pm 5 \mathrm{GeV}$ of the $\tilde{\chi}_{1}^{0}$ peak reconstructed in the previous section were selected, and the $\tilde{\chi}_{1}^{0}$ was combined with another lepton with $p_{\mathrm{T}}>10 \mathrm{GeV}$ and $|\eta|<2.5$. The resulting mass distribution, Figure 20-78, shows a peak at the $\tilde{l}_{L}$ mass, $203 \mathrm{GeV}$, with a fitted width of 1.3 $\mathrm{GeV}$. In addition to the resonance peak, there is also a low-mass structure in Figure 20-78. This results from $\tilde{\chi}_{1}^{ \pm} \rightarrow \tilde{v} l \rightarrow \tilde{\chi}_{1}^{0} v l \rightarrow \tilde{l}_{R}^{ \pm} l^{\mp} v l$, which has a kinematic endpoint at

$$
\sqrt{M_{\tilde{\chi}_{1}^{ \pm}}^{2}-M_{v}^{2}+M_{\tilde{\chi}_{1}^{0}}^{2}}=134 \mathrm{GcV} \text {. }
$$

While this endpoint is clearly visible, there is a lot of background under it, so an accurate measurement will not be easy. Nevertheless, this structure will provide some measurement of a combination of the $\tilde{v}$ and $\tilde{\chi}_{1}^{ \pm}$masses.

\subsubsection{Reconstruction of squarks}

At Point G2b squarks are considerably lighter than gluinos. Direct production of squarks dominates, and the branching ratio for $\tilde{q}_{R} \rightarrow \tilde{\chi}_{1}^{0} q$ is about $95 \%$. Events were selected to have an $\tilde{l}^{ \pm} l^{\mp}$ mass within $\pm 5 \mathrm{GeV}$ of the $\tilde{\chi}_{1}^{0}$ peak in Figure 20-76, and the $\tilde{l}^{ \pm} l^{\mp}$ was then combined with each one of the four hardest jets in the event. The resulting $\bar{\chi}_{1}^{0} j$ mass distribution, Figure 20-79, shows a peak about $2 \%$ below the average $\tilde{q}_{R}$ mass of $648 \mathrm{GeV}$ with a

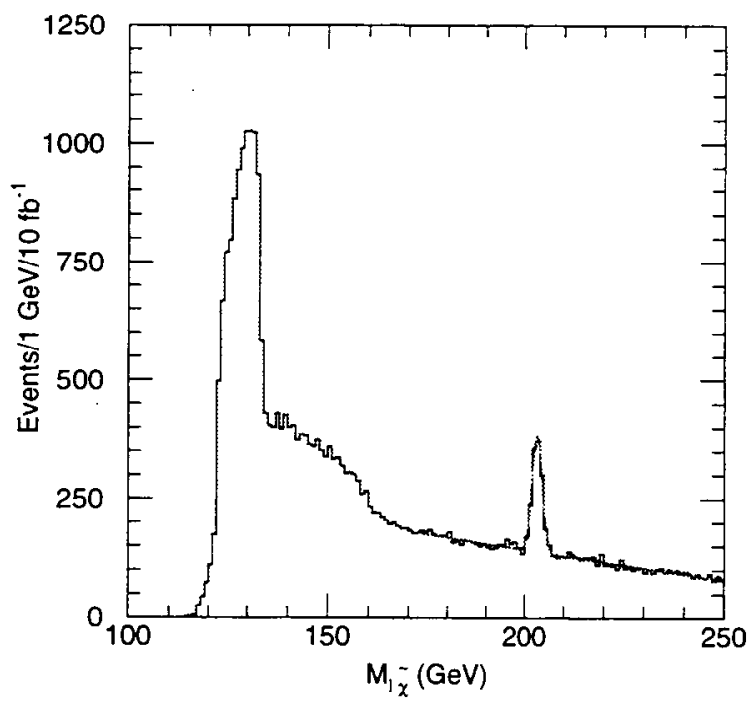

Figure 20-78 Mass distribution of a slepton and two leptons at Point G2b. The slepton and one lepton are required to be within $\pm 5 \mathrm{GeV}$ of the $\tilde{\chi}_{1}^{0}$ peak in Figure 20-76 width of about $26 \mathrm{GeV}$. The statistical error on the mass is negligible; the mass shift could be corrected by applying the jet rescaling procedures described in Section 9.1.1.3 and the mass measured to about $\pm 1 \%$, the systematic limit on the jet energy scale. The contribution of jets tagged as $b$ 's, shown as the dotted curve in the figure, is very small.

The branching ratio for $\tilde{q}_{L} \rightarrow \tilde{\chi}_{2}^{0} q$ is about $25 \%$. This decay can be reconstructed by in a similar way by selecting events with an $\tilde{l}^{ \pm} l^{\mp}$ mass within $\pm 5 \mathrm{GeV}$ of the $\tilde{\chi}_{2}^{0}$ peak in Figure 20-76 and then combining the $\tilde{l}^{ \pm} l^{\mp}$ with any of the four hardest jets. The resulting $\tilde{\chi}_{2}^{0} j$ mass distribution, Figure $20-80$, shows a peak about $3 \%$ below the average $\tilde{q}_{L}$ mass of $674 \mathrm{GeV}$. The figure also shows the distribution for the subset of events tagged as $b$ 's. (No correction to the $b$-jet energy scale was made.) In contrast to the $\tilde{\chi}_{1}^{0} j$ case, there is a clear peak close to the average $\tilde{b}$ mass $647 \mathrm{GeV}$ with a splitting of only $9 \mathrm{GeV}$ - which is significantly below the $\tilde{q}_{L}$ mass. While the de- 


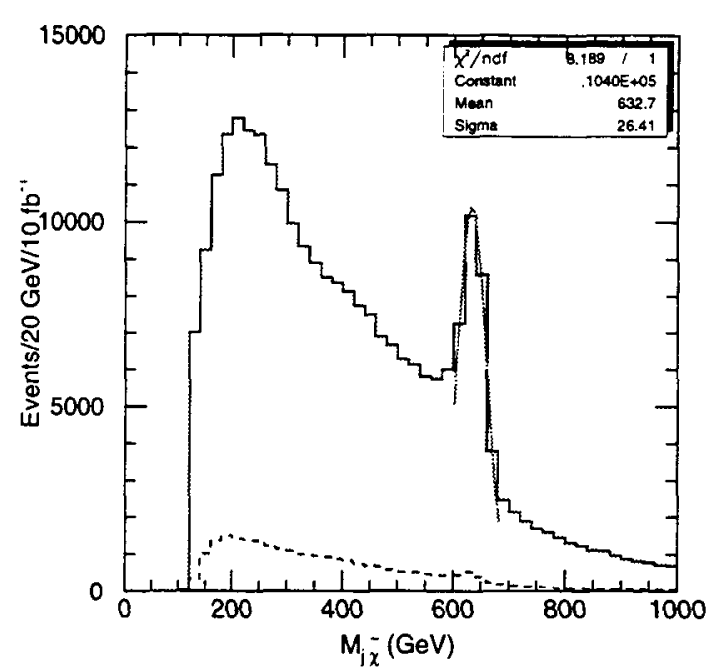

Figure 20-79 $\bar{\chi}_{1}^{0} j$ mass distribution at Point G1b. The $\tilde{l}^{ \pm} l^{F}$ is required to be within $\pm 5 \mathrm{GeV}$ of the $\tilde{\chi}_{1}^{0}$ peak in Figure 20-76. The dashed curve is for jets which are tagged as $b$ 's.

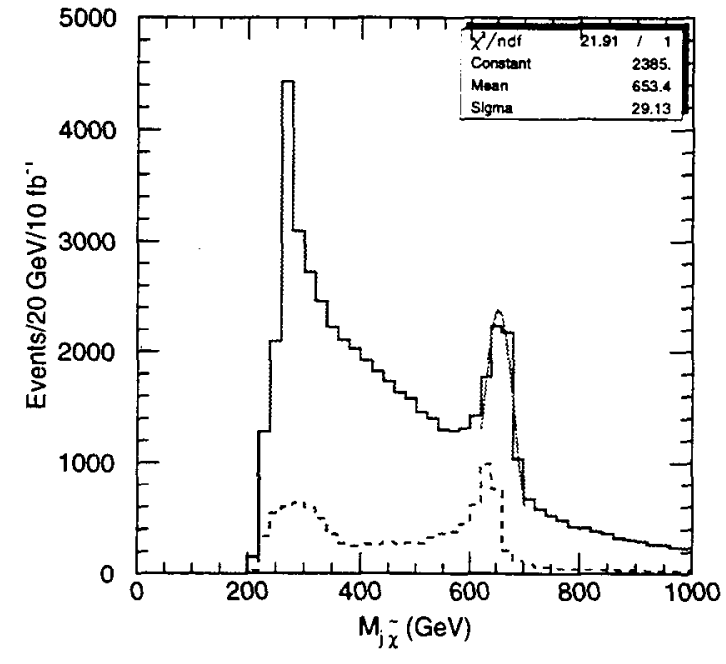

Figure 20-80 Same as Figure 20-79 but for $\tilde{\chi}_{2}^{0} j$.

cay $\tilde{q}_{R} \rightarrow \tilde{\chi}_{1}^{0} q$ is dominant, both the $\tilde{b}_{1}$ and the $\tilde{b}_{2}$ have branching ratios of only a few percent into $\tilde{\chi}_{1}^{0}$. The $\tilde{b}$ contribution explains the fact that the difference between the fitted masses in Figures 20-79 and 20-80 is a factor of two smaller than the $\tilde{q}_{L}-\tilde{q}_{R}$ mass difference.

\subsubsection{Reconstruction of $\tilde{\tau} \tau$ decays}

While $\tilde{\chi}_{i}^{0} \rightarrow \tilde{\tau} \tau$ is more difficult to reconstruct than $\tilde{\chi}_{i}^{0} \rightarrow \tilde{l} l$, it provides additional information, e.g., about the Higgsino content of the neutralinos. The analysis described here is only a first pass: it uses generator information to identify the $\tau$ 's and their visible decay products rather than the more sophisticated and realistic analysis used in Section 20.2.8. Hadronic $\tau$ 's were selected with $p_{\mathrm{T}}>20 \mathrm{GeV},|\eta|<2.5$, and either one or three charged tracks. A (perhaps optimistic) efficiency of $60 \%$, the same as for $b$-tagging, was assumed and is included. The visible $\tau$ momentum was then combined with the slepton momentum. The resulting $\tilde{\tau} \tau$ distribution is shown in Figures $20-81$ and $20-82$ on two different scales. While the distribution does not show any mass peaks because of the missing neutrinos, it does have rather sharp endpoints at the $\tilde{\chi}_{1}^{0}$ and $\tilde{\chi}_{2}^{0}$ masses.

If there is only one $v_{\tau}$, then the true $E_{T}^{\text {miss }}$ calculated from the calorimeter plus the sleptons can be used to determine the $\tau$ momentum. Only the $\tau$ with the highest $p_{\Upsilon}$ is used, and the angle between it and the $E_{\mathrm{T}}^{\text {miss }}$ direction is required to be $\Delta \phi<0.1 \pi$. The visible $\tau$ momentum is then scaled by a factor $1+E_{\mathrm{T}}^{\text {miss }} / E_{\mathrm{T}, \tau}$, and the $\tilde{\tau} \tau$ mass is recomputed. This gives the dashed curves in Figures 20-81 and 20-82. As expected, including $E_{\mathrm{T}}^{\text {miss }}$ not only reduces the statistics but also worsens the resolution for the $\tilde{\chi}_{1}^{0}$, since the $\tau$ from $\tilde{\chi}_{1}^{0}$ is very soft. However, it produces a peak near the right position for the $\tilde{\chi}_{2}^{0}$. While this peak probably does not improve the mass resolution, it adds confidence that one is seeing a two-body resonance. 


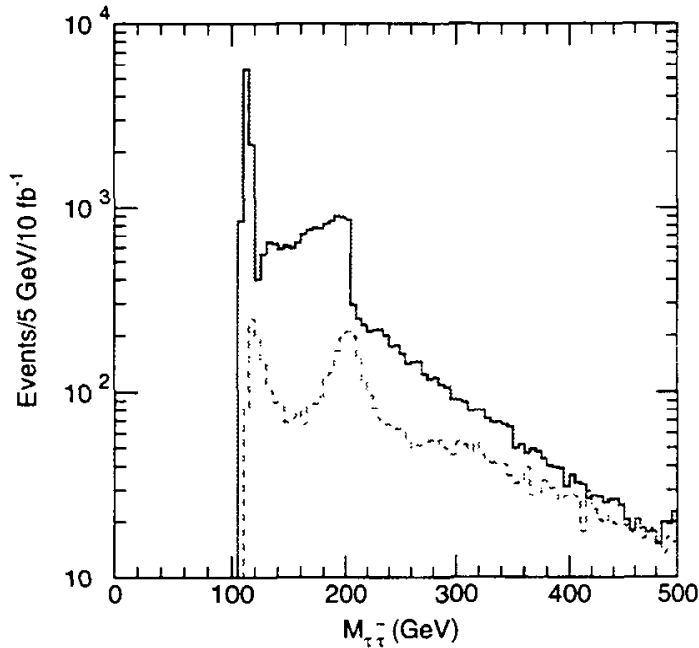

Figure 20-81 $\tilde{\tau} \tau$ invariant mass distribution at Point G2b. Solid curve: using visible $\tau$ momentum. Dashed curve: using $E_{\mathrm{T}}^{\text {miss }}$ as described in the text.

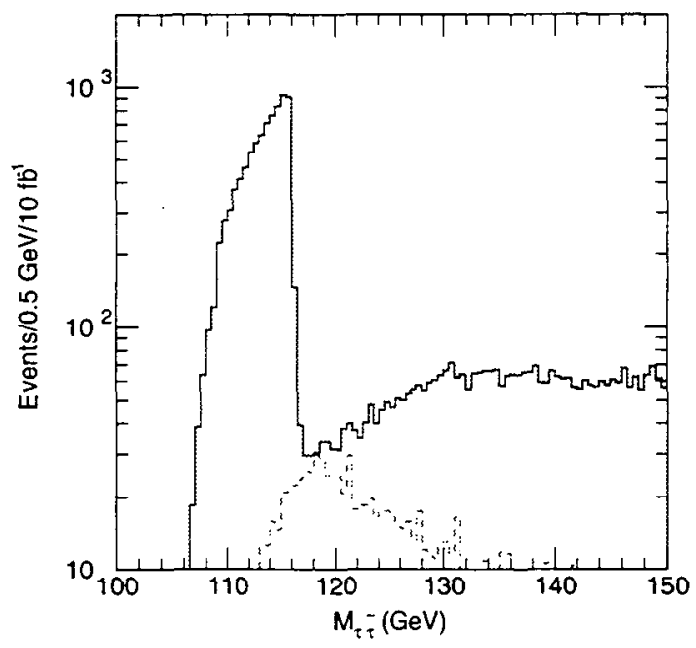

Figure 20-82 Same as Figure 20-81 on a finer scale.

\subsubsection{Direct production of sleptons and gauginos}

The peak at low $M_{\text {eff }}$ in Figure 20-74 is due to the direct production of sleptons and gauginos. Studying this direct production does not lead to reconstruction of any new masses, but does contain information that could be used to constrain the SUSY model. Events were selected as in Section 20.3.4.3 and Figure 20-76 with the additional requirement $M_{\text {eff }}<100 \mathrm{GeV}$, where the effective mass of course excludes the sleptons. The resulting $\tilde{l l}$ mass distribution, Figure 20-83,

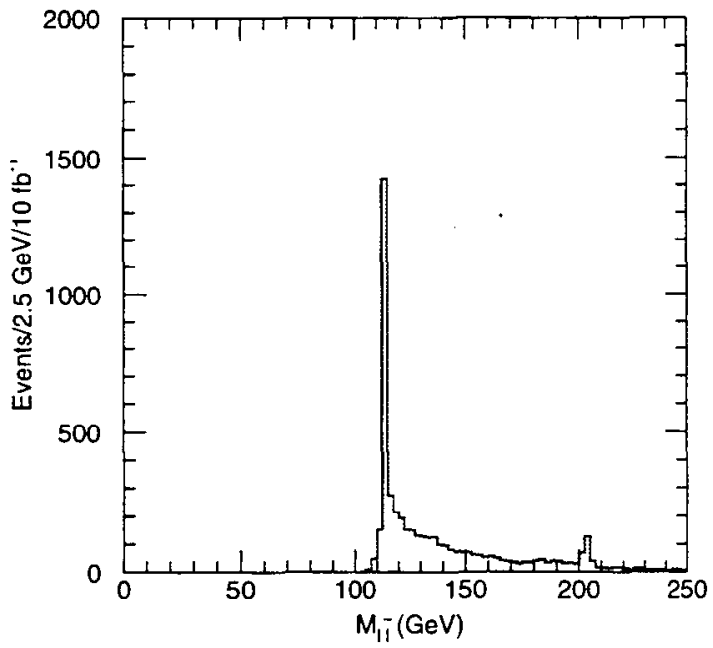

Figure 20-83 Same as Figure 20-76 with the additional requirement $M_{\text {eff }}<100 \mathrm{GeV}$.

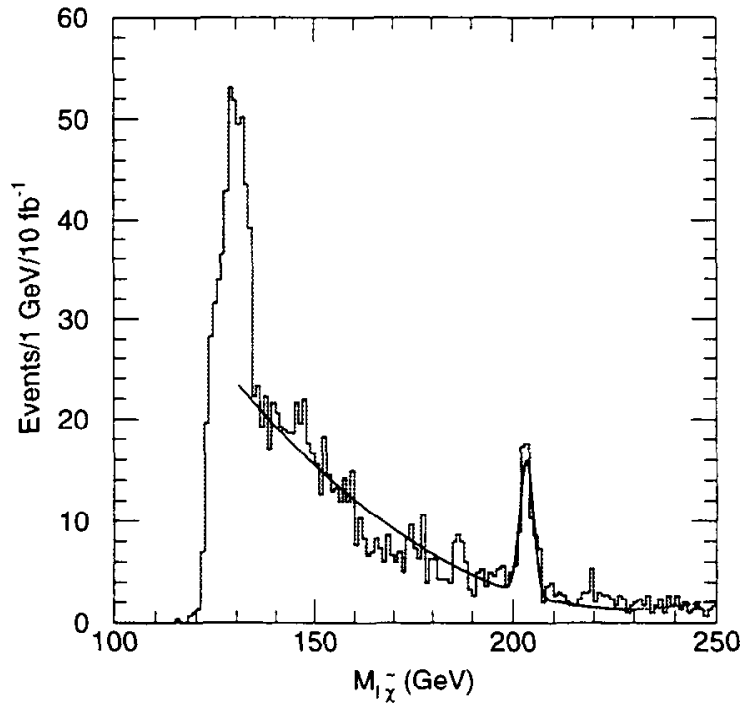

Figure 20-84 Combination of $\tilde{l} l$ from Figure 20-83 in the mass range $110-120 \mathrm{GeV}$ with an additional lepton. 
shows a strong $\tilde{\chi}_{1}^{0}$ peak and a small $\tilde{\chi}_{2}^{0}$ peak. The second peak is suppressed both by its larger mass and by the fact that its decay products can contribute to $M_{\text {eff }}$, causing part of the signal to be discarded.

Events from the $\tilde{\chi}_{1}^{0}$ peak in Figure 20-83 can be combined with a third lepton to reconstruct directly produced sleptons decaying via $\tilde{l}_{L} \rightarrow \tilde{\chi}_{1}^{0} l \rightarrow \tilde{l}_{R}^{ \pm} l^{\mp} l$. Events with $110<M_{\tilde{l}}<120 \mathrm{GeV}$ were combined with another lepton having $p_{\mathrm{T}}>10 \mathrm{GeV}$ and $|\eta|<2.5$. Figure 20-84 shows the resulting mass distribution with a slepton peak having a fitted mass of $203.3 \mathrm{GeV}$ and a width of $1.66 \mathrm{GeV}$. The low-mass feature from $\tilde{\chi}_{1}^{ \pm} \rightarrow \tilde{v} l \rightarrow \tilde{\chi}_{1}^{0} v l \rightarrow \tilde{l}_{R}^{ \pm} l^{\mp} v l$ discussed previously is also visible. The number of events is much lower than in Figure 20-78, showing that the inclusive signals reconstructed in Section 20.3.4.4 are dominated by cascade decays, not by direct production.

\subsubsection{Fitting GMSB parameters}

In several of the GMSB cases considered a number of masses can be determined from the kinematic properties of the event without making use of a model. Nevertheless, it is still useful to make a global fit to determine the parameters of the minimal GMSB model. The method is similar to that described in Section 20.2.9. A scan is made over the ranges

$$
\begin{gathered}
\Lambda>1000 \mathrm{GeV} \\
M_{m} \geq \Lambda \\
1 \leq N_{5} \leq 10 \\
1<\tan \beta<20
\end{gathered}
$$

for both signs of $\mu$. The upper limits on $\Lambda$ and $M_{m}$ are determined iteratively for each point. Once an approximate solution has been determined, the ranges are reduced and more points are scanned iteratively until the $\pm 1 \sigma$ errors are determined. The signatures for the GMSB points are qualitatively different from any SUGRA model except for Point G1b; no minimal SUGRA solution was found for that point.

Results of the fits [20-36] for these four parameters are given for the same Low-L, High-L, and Ultimate scenarios as in Section 20.2.9 except that for the first two the theoretical error on the light Higgs mass is taken to be $\pm 3 \mathrm{GeV}$, somewhat more conservative than the value assumed in the SUGRA fits. The parameter $C_{\mathrm{grav}}$ is independent from the other parameters, and is determined in each case from the NLSP lifetime as already discussed. No study of the determination of non-minimal GMSB parameters has yet been made.

\subsubsection{Point G1a}

At Point Gla the presence of two prompt photons in almost every SUSY event strongly suggests a GMSB-like scenario. Besides the light Higgs mass there are four precisely measured combinations of leptons and photons, which are summarised in Table 20-18 with their estimated errors. The estimated error on $M_{l l}^{\max }$ at low luminosity may be a bit too small compared to the errors given in Section 20.2.3. The errors at high luminosity are dominated by systematic effects. These four observables can all be related to combinations of the masses involved, and they are sufficient to determine the parameters of the minimal model. The other measured quantities provide cross checks on the model but do not significantly constrain the parameters. 
Table 20-18 Inputs for the minimal GMSB fit at Point G1a.

\begin{tabular}{llll}
\hline Quantity & Reference & Low-L & Ultimate \\
\hline$M_{h}$ & Section 19.2 .2 & $(109.47 \pm 3.0) \mathrm{GeV}$ & $(109.27 \pm 0.2) \mathrm{GeV}$ \\
$M_{l l}^{\max }$ & Section 20.3 .1 .1 & $(105.1 \pm 0.1) \mathrm{GeV}$ & same \\
$M_{l l \gamma}^{\max }$ & Section 20.3 .1 .1 & $(189.7 \pm 0.3) \mathrm{GeV}$ & $(189.7 \pm 0.2) \mathrm{GeV}$ \\
$M_{l \gamma}^{(1)}$ & Section 20.3.1.1 & $(112.7 \pm 0.15) \mathrm{GeV}$ & $(112.7 \pm 0.1) \mathrm{GeV}$ \\
$M_{l \gamma}^{(2)}$ & Section 20.3.1.1 & $(152.6 \pm 0.3) \mathrm{GeV}$ & $(152.6 \pm 0.2) \mathrm{GeV}$ \\
\hline
\end{tabular}

Table 20-19 Results of the minimal GMSB fit at Point G1a. $\operatorname{sgn} \mu=+$ is unambiguously determined. Note that the errors in Reference [20-36] are too small by a factor of $\sqrt{2}$.

\begin{tabular}{llll}
\hline Parameter & Low-L & High-L & Ultimate \\
\hline$\Lambda$ & $(90000 \pm 1700) \mathrm{GeV}$ & $(90000 \pm 890) \mathrm{GeV}$ & same \\
$M_{m}$ & $(500000 \pm 170000) \mathrm{GeV}$ & $(500000 \pm 110000) \mathrm{GeV}$ & same \\
$N_{5}$ & $1.00 \pm 0.014$ & $1.00 \pm 0.011$ & same \\
$\tan \beta$ & $5.0 \pm 1.3$ & $5.0 \pm 0.4$ & $5.0 \pm 0.14$ \\
\hline
\end{tabular}

The results of the fit are shown in Table 20-19. As expected, $\Lambda, N_{5}$, and $\operatorname{sgn} \mu$ are well determined even at low luminosity, while $M_{m}$ is never very precisely determined because it enters only through the logarithmic running of masses in the renormalisation group equations. Since $\tan \beta$ is determined mainly from the Higgs mass, the errors on it do improve. Another handle on $\tan \beta$ might be provided by the large violation of $e / \mu / \tau$ universality at Point Gla, e.g.,

$$
\begin{aligned}
& B\left(\tilde{\chi}_{2}^{0} \rightarrow \tilde{e} e\right)=25 \% \\
& B\left(\tilde{\chi}_{2}^{0} \rightarrow \tilde{\tau} \tau\right)=41 \%
\end{aligned}
$$

This has not yet been studied.

\subsubsection{Point G1b}

While the masses are the same at Point G1b as at Point Gla, the absence of $\tilde{\chi}_{1}^{0} \rightarrow G \gamma$ decays leads to fewer measurements, which are summarised in Table 20-20. The measurement of the average slepton mass relies [20-36] on understanding the shape of a lepton-jet mass distribution, not just on kinematics. The gluino mass measurement nominally comes from an endpoint, but it also really requires understanding the shape of a distribution.

If the squark mass measurement is not included, then the fit is only able to constrain two combinations of parameters with any precision. For $30 \mathrm{fb}^{-1}$,

- $\Lambda N_{5}=(90000 \pm 880) \mathrm{GeV}$;

- $\tan \beta=5.0_{-1.8}^{+2.7}$.

There is no independent information on $\Lambda$ and $N_{5}$ because the position of the dilepton edge is independent of the slepton mass for $M_{\tilde{l}_{R}}=\sqrt{M_{\bar{\chi}_{1}^{0}} M_{\bar{\chi}_{2}^{0}}}$, and the equality holds to within $0.5 \mathrm{GeV}$ at this point. 
Table 20-20 Inputs for the minimal GMSB fit at Point G1b. The measurement below the horizontal rule determines a combination of masses and production dynamics.

\begin{tabular}{llll}
\hline Quantity & Reference & Low-L & Ultimate \\
\hline$M_{h}$ & Section 19.2.2 & $(109.47 \pm 3.0) \mathrm{GeV}$ & $(109.27 \pm 0.2) \mathrm{GeV}$ \\
$M_{l l}^{\max }$ & Section 20.3.2.2 & $(105.1 \pm 0.1) \mathrm{GeV}$ & same \\
$M_{\tilde{g}}-M_{\tilde{\chi}_{2}^{0}}$ & Section 20.3.2.3 & $(523 \pm 30) \mathrm{GeV}$ & $(523 \pm 15) \mathrm{GeV}$ \\
\hline$\left\langle M_{\tilde{q}_{L}}\right\rangle$ & {$[20-36]$} & $(988 \pm 50) \mathrm{GeV}$ & same \\
\hline
\end{tabular}

If the squark mass measurement is included, then the fit gives for $30 \mathrm{fb}^{-1}$

- $\Lambda N_{5}=(90000 \pm 880) \mathrm{GeV}$;

- $\Lambda=(90000 \pm 11500) \mathrm{GeV}$;

- $M_{m}<7 \times 10^{8} \mathrm{GeV}$;

- $\tan \beta=5.0_{-1.8}^{+2.7}$;

assuming $\operatorname{sgn} \mu=+$, but there is also a solution for $\operatorname{sgn} \mu=-$. This can be eliminated only if the slepton mass can be measured independently or the squark mass can be measured to $\pm 10 \mathrm{GeV}$. The errors decrease somewhat with higher luminosity, but the GMSB parameters remain poorly constrained.

The signatures for Point G1b are not qualitatively different from those of a SUGRA model. A fit to the measurements in Table 20-20 not including the squark mass gives for $30 \mathrm{fb}^{-1}$

- $m_{0}=(100 \pm 20) \mathrm{GeV}$;

- $m_{1 / 2}=(295 \pm 6) \mathrm{GeV}$;

- $\tan \beta=4.5 \pm 1.1$;

- $\operatorname{sgn} \mu=+$;

- $A_{0}=(250 \pm 200) \mathrm{GeV}$.

The fit has a probability of only $15 \%$. The mean value of the squark mass is $760 \mathrm{GeV}$; GMSB models typically give a more spread-out spectrum than SUGRA ones. Hence the SUGRA fit can be ruled out by the squark measurement.

It may be possible to obtain a better determination of the parameters at this point, but doing so would require generating many samples of events and comparing the predicted distributions with the data. Thus, this point serves as a caution about drawing overly optimistic conclusions about the generality of the methods discussed in this chapter.

\subsubsection{Point G2a}

At Point G2a the prompt decay of the slepton provides well-measured multi-step decays. The measurements summarised in Table 20-21 are already limited by systematics for an integrated luminosity of $30 \mathrm{fb}^{-1}$; there is indeed not much improvement beyond $10 \mathrm{fb}^{-1}$. All of the measurements can be related precisely to combinations of masses, making the fit straightforward. 
Table 20-21 Inputs for the minimal GMSB fit at Point G2a.

\begin{tabular}{llll}
\hline Quantity & Reference & Low-L & Ultimate \\
\hline$M_{h}$ & Section 19.2.2 & $(106.6 \pm 3.0) \mathrm{GeV}$ & $(106.6 \pm 0.2) \mathrm{GeV}$ \\
$M_{l l}^{\max }$ (first edge) & Section 20.3.3.1 & $(52.21 \pm 0.05) \mathrm{GeV}$ & same \\
$M_{l l}^{\max }$ (second edge) & Section 20.3.3.1 & $(175.94 \pm 0.18) \mathrm{GeV}$ & same \\
$M_{l l q}^{\max }$ & Section 20.3.3.2 & $(640 \pm 7) \mathrm{GeV}$ & same \\
$M_{l q}^{\max } / M_{l l q}^{\max }$ & Section 20.3.3.2 & $0.450 \pm 0.004$ & same \\
\hline
\end{tabular}

Table 20-22 Results of the minimal GMSB fit at Point G2a. $\operatorname{sgn} \mu=+$ is unambiguously determined. Note that the errors in Reference [20-36] are too small by a factor of $\sqrt{2}$.

\begin{tabular}{llll}
\hline Parameter & Low-L & High-L & Ultimate \\
\hline$\Lambda$ & $(30000 \pm 540) \mathrm{GeV}$ & same & same \\
$M_{m}$ & $(250000 \pm 60000) \mathrm{GeV}$ & same & same \\
$N_{5}$ & $3.00 \pm 0.05$ & same & same \\
$\tan \beta$ & $5.0 \pm 1.0$ & $5.0 \pm 1.0$ & $5.0 \pm 0.06$ \\
\hline
\end{tabular}

The results of the fit are summarised in Table 20-22. Since there is accurate information on both sleptons and gauginos, $\Lambda$ and $N_{5}$ are well determined even for low luminosity, and even $M_{m}$ is determined to about $25 \%$. The sign of $\mu$ is unambiguous. The reduced error on $\tan \beta$ for the Ultimate fit comes just from assuming no theoretical error on the Higgs mass.

\subsubsection{Point G2b}

At Point G2b the sleptons are stable; their masses can be determined by time of flight measurements and their momenta directly measured. This allows many different masses to be reconstructed directly as peaks in invariant mass distributions Even for an integrated luminosity of $10 \mathrm{fb}^{-1}$ the errors are limited by the systematics of the detector energy scales, assumed to be $1 \%$ for jets and $0.1 \%$ for electrons and muons, so there is no need to summarise them here.

The results of the fit are summarised in Table 20-23. Again, $\Lambda$ and $N_{5}$ are well determined. $M_{m}$ is determined to less than $\pm 15 \%$, and the error on $\tan \beta$ is limited by the uncertainty on the light Higgs mass. At least within the minimal GMSB model, the only question is how well $C_{\text {grav }}$ could be measured or limited by looking for occasional slepton decays in the tracking system.

Table 20-23 Results of the minimal GMSB fit at Point G2b. $\operatorname{sgn} \mu=+$ is unambiguously determined. Note that the errors in Reference [20-36] are too small by a factor of $\sqrt{2}$.

\begin{tabular}{llll}
\hline Parameter & Low-L & High-L & Ultimate \\
\hline$\Lambda$ & $(30000 \pm 250) \mathrm{GeV}$ & same & same \\
$M_{m}$ & $(250000 \pm 32000) \mathrm{GeV}$ & same & same \\
$N_{5}$ & $3.00 \pm 0.02$ & same & same \\
$\tan \beta$ & $5.0 \pm 0.3$ & $5.0 \pm 0.3$ & $5.0 \pm 0.03$ \\
\hline
\end{tabular}


The signature is straightforward in principle - the $\tilde{l}$ track ends, and an $l$ track begins with a relative $p_{T}-M_{\tilde{l}} / 2$, giving rise to 'kinks' - but the pattern recognition has not yet been studied. It however should be feasible at low luminosity.

\section{$20.4 R$-Parity breaking models}

The conservation of $R$-parity, defined as

$$
R=(-1)^{3(B-L)+2 S}
$$

is an elegant way of imposing at the same time baryon $(B)$ and lepton $(L)$ number conservation in supersymmetric theories. However, there is no compelling theoretical reason why $R$-invariance should be a symmetry of the Lagrangian. It is therefore useful to investigate the phenomenological consequences of realistic models with broken $R$-parity.

In models with minimal field content, the $R$-violating superpotential can be written as

$$
W_{R P V}=\lambda_{i j k} L_{i} L_{j} E_{k}^{c}+\lambda^{\prime}{ }_{i j k} Q_{i} L_{j} D_{k}^{c}+\lambda^{\prime \prime}{ }_{i j k} U_{i}^{c} D_{j}^{c} D_{k}^{c}
$$

where $L$ and $E$ are respectively isodoublet and isosinglet lepton, and $Q$ and $D$ are isodoublet and isosinglet quark superfields, and the indices $i, j, k$ run over the three quark and lepton generations. The superscript $c$ indicates charge conjugation. The terms in the superpotential explicitly violate baryon number (through the nine $\lambda^{\prime \prime}{ }_{i j k}$ couplings) and lepton number (by the nine $\lambda_{i j k}$ and the $27 \lambda_{i j k}^{\prime}$ couplings). In order to ensure proton stability, either $L$ or $B$ violating terms should be absent. Experimental limits on various $L$ - and $B$-violating processes constrain the values of most of the $\lambda$ couplings to a few $10^{-2}$ [20-14], but some of the couplings have bounds of order one.

Any additional term in the Lagrangian with a coupling bigger than $10^{-2}$ becomes competitive with the gauge couplings and affects the mass spectra and the branching ratios of the models, which will be very different from the $R$-conserving case. Such scenarios, for which no appropriate simulation tool was available for $p p$ physics, are not considered further in this study. (Work on such scenarios has now started using HERWIG [20-40].) For lower values of the $\lambda$ couplings, the only effect is the fact that the LSP becomes unstable, and decays to three $R$-even particles. The search strategies are thus drastically different with respect to $R$-parity conserving models, for which the basic signature is the $E_{T}^{\text {miss }}$ from undetected $\tilde{\chi}_{1}^{0}$ in the final state.

For a value of the coupling constant $\lambda<10^{-6}$ the LSP will decay outside the detector, thus giving a phenomenology identical to $R$-parity conserving models. For $\lambda$ values between $10^{-2}$ and $10^{-6}$ the LSP will either decay with a displaced vertex in the detector, or at the interaction vertex. The studies in this section focus on the latter case, for which no additional information is available to disentangle the LSP decay products from the Standard Model background.

Given the requirements from proton decay lifetime, only one of the three terms in the superpotential is assumed to be non-zero for each given model study. Each of three cases presents a distinct $\tilde{\chi}_{1}^{0}$ decay pattern:

- $\tilde{\chi}_{1}^{0} \rightarrow q q q$ for $\lambda^{\prime \prime}{ }_{i j k} \neq 0$ giving six additional jets in the final state.

- $\tilde{\chi}_{1}^{0} \rightarrow l^{+} l^{-} \vee$ for $\lambda_{i j k} \neq 0$ giving four charged leptons per event and $E_{\mathrm{T}}^{\text {miss }}$.

- $\tilde{\chi}_{1}^{0} \rightarrow q \bar{q} l, q \bar{q} \vee$ for $\lambda_{i j k}^{\prime} \neq 0$ giving four additional jets and two charged leptons or $E_{\mathrm{T}}^{\mathrm{miss}}$. 
Each of the cases was studied separately, in general for each case assuming that the coupling constant is non zero only for one flavour combination at a time.

All the studies were performed within the minimal SUGRA model, with the $\tilde{\chi}_{1}^{0}$ forced to decay to the appropriate quark/lepton combination. The first step in the study was to verify that SUSY events can still be sorted out from the Standard Model background, even in the absence of the classic $E_{T}^{\text {miss }}$ signature. This is straightforward for the $L$-violating couplings, which in most of the cases produce a high number of leptons in the final state, and requires a more careful analysis for the $B$-violating case. The next step was to study, for the five sample points in the SUGRA parameter space, the same exclusive decay chains that were studied for the $R$-conserving case. In general, the increased complexity of the events will make it more difficult to extract exclusive signatures from the SUSY combinatorial background. On the other hand, in many cases it will be possible to reconstruct the $\bar{\chi}_{1}^{0}$ from its decay products, opening the possibility of the full reconstruction of the masses of the particles taking part in the identified decay chains.

\subsubsection{Baryon number violation: $\chi_{1}{ }^{0} \rightarrow q q q$}

This is potentially the most difficult case, as the decay of the $\tilde{\chi}_{1}^{0}$ into three jets destroys the $E_{\mathrm{T}}^{\text {miss }}$ signature, and the increased jet multiplicity may not give a sufficient handle to extract the SUSY signal from the Standard Model background. The choice of a specific $\lambda^{\prime \prime}{ }_{i j k}$ as the dominant coupling only affects the final state signatures by changing the heavy quark multiplicity in the events. Two independent studies were performed using ISAJET [20-41] and HERWIG 6.0 [20-42], assuming that only the $R$-violating coupling $\lambda^{\prime \prime}{ }_{212}$ is different from zero, yielding the decay

$$
\tilde{\chi}_{1}^{0} \rightarrow c d s
$$

plus its complex conjugate. This particular choice of the non-zero coupling is motivated by the absence of significant experimental bounds [20-14] and by the decision to study a particularly difficult case where the high number of $c$-quarks in the final states increases the background to signatures based on the detection of $b$-quarks. HERWIG 6.0 contains a preliminary version of a simulation of all $R$-parity violating processes [20-40].

The general features of the model were studied using SUGRA Point 5 as an example. In Figures 20-85 and 20-86 the distributions of $E_{\mathrm{T}}^{\mathrm{miss}}$ and of the number of jets with $p_{\mathrm{T}}>15 \mathrm{GeV}$ are shown for the $R$-conserving case and the $R$-violating case at Point 5 . The $E_{T}^{\text {miss signature has }}$ been significantly reduced, but the jet multiplicity is higher, as expected. The $\tilde{\chi}_{1}^{0}$ has a rather low mass, $112 \mathrm{GeV}$, and the jets from its decay will be rather soft and not well separated.

\subsubsection{Inclusive signatures}

The SUSY cross-section at SUGRA Point 5 is dominated by squark production either directly or from gluino decay. The final states will therefore be characterised by the presence of at least eight hadronic jets, and by a mass scale around $700 \mathrm{GeV}$. In order to have an estimate of the mass scale a variable can be defined:

$$
m_{\mathrm{T}, \text { cent }}=\sum_{(\eta<2)} p_{\mathrm{T}}^{\text {jet }}+\sum_{(\eta<2)} p_{\mathrm{T}}^{\text {lepton }} .
$$

The distribution of $m_{\mathrm{T}}$, cent for events selected by requiring: 


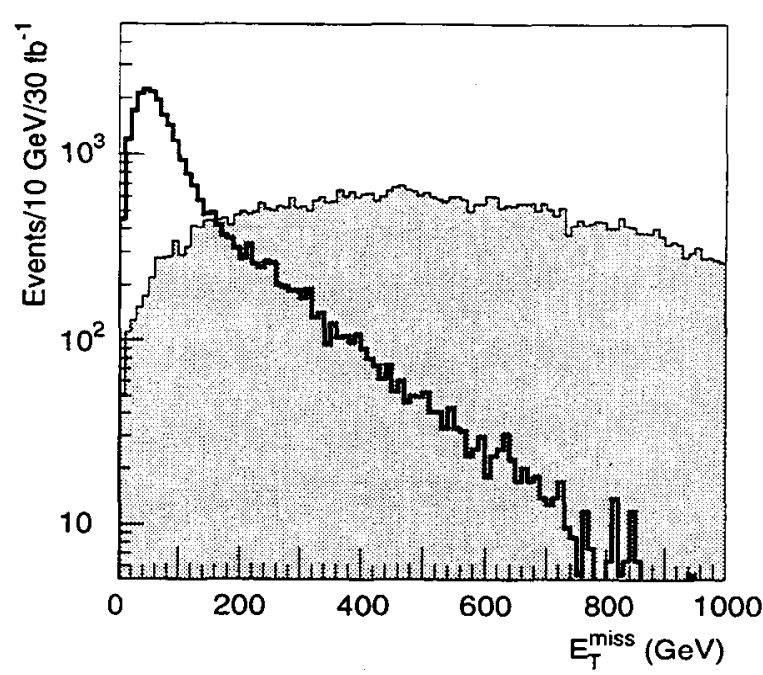

Figure 20-85 $E_{\mathrm{T}}^{\mathrm{miss}}$ distribution for SUGRA Point 5 in the case of R-parity conservation (shaded histogram) and $R$-parity violation (empty histogram).

- at least eight jets with $p_{\mathrm{T}}>50 \mathrm{GeV}$;

- at least one jet with $p_{\mathrm{T}}>100 \mathrm{GeV}$;

- transverse sphericity $>0.2$, thrust $>0.9$;

is shown in Figure 20-87 for the SUSY signal, for QCD jets, and for other Standard Model backgrounds. A hard cut on the jet $p_{\mathrm{T}}$ is necessary to reduce the $Q C D$ multijet background, but it also reduces the efficiency for the jets from the $\bar{\chi}_{1}^{0}$ decay. After the cuts described above, the signal is still dominated by the QCD background for all values of $m_{\mathrm{T} \text {, cent }}$.

The SUSY signal can therefore be separated from the SM background only by requiring the presence of at least one lepton in the event. If eight jets with $p_{\mathrm{T}}>25 \mathrm{GeV}$ and a lepton with $p_{\mathrm{T}}>15 \mathrm{GeV}$ are required, a cut on $m_{\mathrm{T} \text {, cent }}>1000 \mathrm{GeV}$ will yield a signal over background ratio of 2.4. If two leptons are required, the ratio is greater than ten, and approximately 10000 events are expected for SUGRA Point 5, for an integrated luminosity of $30 \mathrm{fb}^{-1}$.

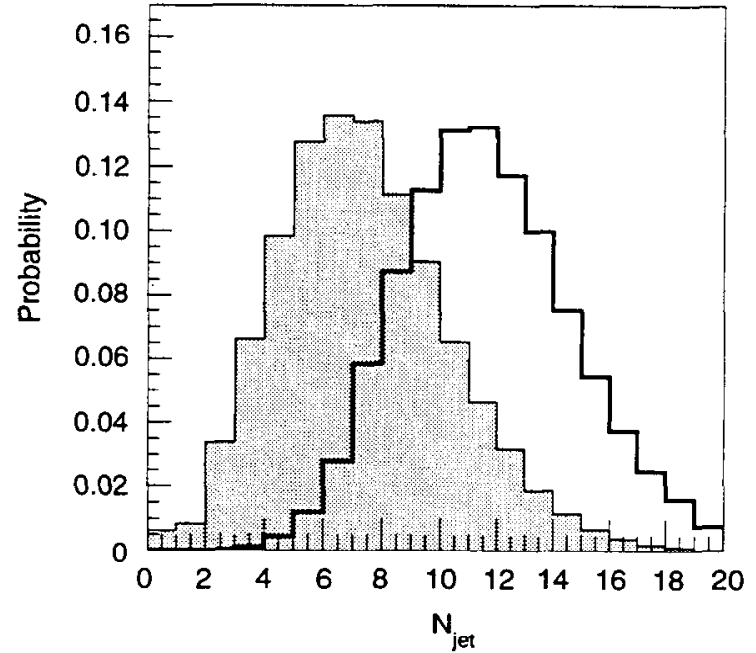

Figure 20-86 Total jet multiplicity $(p \mathfrak{f}$ et $>15 \mathrm{GeV}$ ) distribution for $R$-parity conservation (shaded) and $R$ parity violation at SUGRA Point 5 . The jets are reconstructed using a topological algorithm based on joining neighbouring cells.

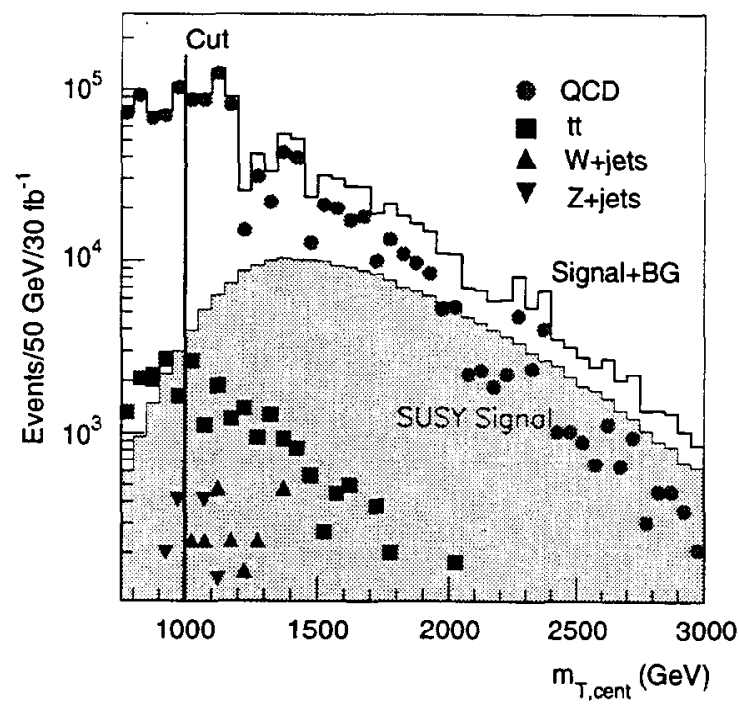

Figure 20-87 $m_{\mathrm{T} \text {, cent }}$ distribution for SUSY signal (shaded histogram), and for signal+background (full line histogram), after requiring at least eight jets with $p_{\mathrm{T}}>50 \mathrm{GeV}$. The different components of the background are also shown separately. 
Another feature of SUGRA Point 5 is the presence of a large number of $b$-jets in the final state. Even by requiring four tagged $b$-jets, with no requirement on leptons, the signal cannot be extracted from the background. The high $b$-jet multiplicity can be used in combination with the requirement of one or more leptons in the final state to confirm a signal for new physics.

Abundant lepton production from $\tilde{\chi}_{2}^{0}$ decays is expected over a large part of the SUGRA parameter space, as shown in Figure 20-6. Therefore, it is expected that it will be possible to discover $B$-violating SUGRA models over a significant fraction of the parameter space with analyses similar to the one performed for Point 5 .

\subsubsection{Precision measurements}

The di-lepton mass edge, produced from the decay $\tilde{\chi}_{2}^{0} \rightarrow \tilde{l}^{ \pm} l^{\mp} \rightarrow \tilde{\chi}_{1}^{0} l^{+} l^{-}$is the starting point for exclusive analyses at SUGRA Point 5 , and provides a very precise constraint on a combination of the masses of $\tilde{\chi}_{1}^{0}, \tilde{\chi}_{2}^{0}$ and $\tilde{l}_{R}$. In $R$ violating models the three masses involved can often be directly measured, but usually with limited statistical precision, and with the systematic uncertainties associated to the use of jets to reconstruct the invariant masses. The detection of the lepton edge provides moreover an easy way of selecting a pure SUSY sample for the reconstruction of exclusive final states.

Events were selected by requiring:

- at least eight jets with $p_{\mathrm{T}}>20 \mathrm{GeV}$;

- transverse sphericity $>0.2$, thrust $>0.9$;

- $m_{\mathrm{T}, \text { cent }}>1000 \mathrm{GeV}$;

- two opposite sign (OS), same flavour (SF) leptons, with $p_{\Upsilon}>10 \mathrm{GeV}$.

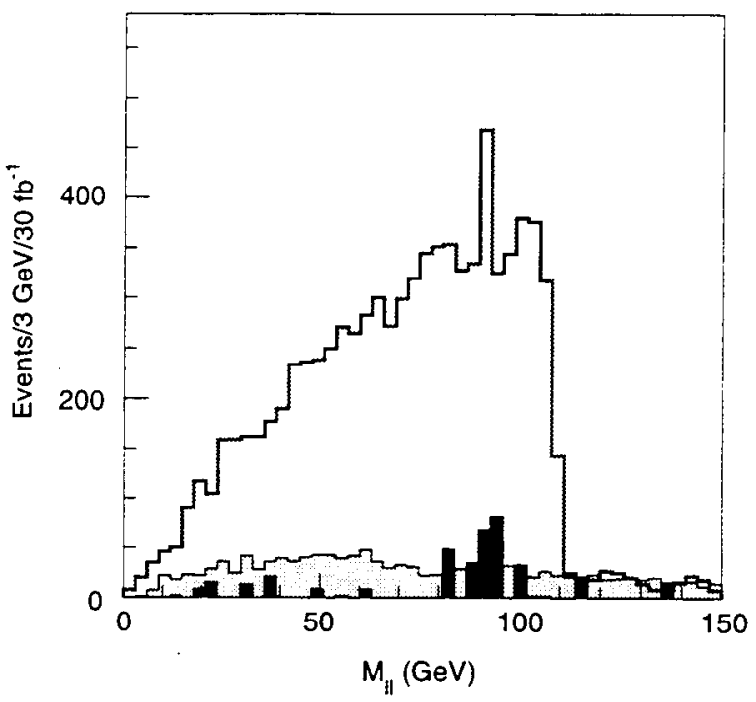

Figure 20-88 Distribution of the invariant mass formed by two OS-SF leptons after the selection described in the text for an integrated luminosity of $30 \mathrm{fb}^{-1}$. The cross-hatched distribution is the SM background, and the hatched distribution is the combinatorial background from OS-DF leptons.

The invariant mass spectrum of the two leptons is shown in Figure 20-88. For an integrated luminosity of $30 \mathrm{fb}^{-1}$ the expected SM background is about 400 events for 7500 SUSY events. The SUSY combinatorial background can be subtracted using the distribution of OS different flavour (DF) events, shown as a hatched histogram in the plot. The edge can be measured as $(109 \pm 0.30$ (stat.) \pm 0.11 (syst.)) $\mathrm{GeV}$, where the systematic error is given by the lepton energy scale.

The direct reconstruction of $\tilde{\chi}_{1}^{0} \rightarrow q q q$ decays is problematic for two main reasons. First there is a high jet multiplicity, produced both from squark and gluino decays and from the presence of two $\tilde{\chi}_{1}^{0} \rightarrow q q q$ decays in each event. Second, the $\tilde{\chi}_{1}^{0}$ mass is typically rather small over most of the accessible SUGRA parameter space $-122 \mathrm{GeV}$ for Point 5 . As an example, the softest jet from the harder (softer) $\tilde{\chi}_{1}^{0}$ has an average $p_{\mathrm{T}}$ of $35(21) \mathrm{GeV}$. It is therefore necessary to keep a very low jet threshold in the analysis, thus increasing the probability of picking up a wrong jet from the underlying event. 
The first step in the reconstruction is aimed at minimising the contribution from additional jets in the event. The high cross-section process with lowest jet multiplicity is $\tilde{q}_{R} \tilde{q}_{L}$ production, followed by the decays $q_{R} \rightarrow \tilde{\chi}_{1}^{0} q$ and $q_{L} \rightarrow \tilde{\chi}_{2}^{0} q$, with the $\tilde{\chi}_{2}^{0}$ decaying via $\tilde{\chi}_{2}^{0} \rightarrow I_{R}^{+} l^{\mp} \rightarrow \tilde{\chi}_{1}^{0} l^{+} l^{-}$. The cuts used to select this process while minimising the QCD background were:

- one or two leptons with $p_{\mathrm{T}}>20 \mathrm{GeV}$. If two, they should be OS-SF with $m_{l l}<109 \mathrm{GeV}$;

- at least eight jets with $p_{\mathrm{T}}>17.5 \mathrm{GeV}$;

- no additional jets and no $b$-jets with $p_{\mathrm{T}}>25 \mathrm{GeV}$;

- two central jets with $|\eta|<2$, and $p_{\mathrm{T}}^{\text {jet } 1}>200 \mathrm{GeV}, p_{\mathrm{T}}^{\text {jet } 2}>100 \mathrm{GeV}$;

- $m_{\mathrm{T}, \text { cent }}>1000 \mathrm{GeV}$;

- transverse sphericity $>0.2$, thrust $>0.9$.

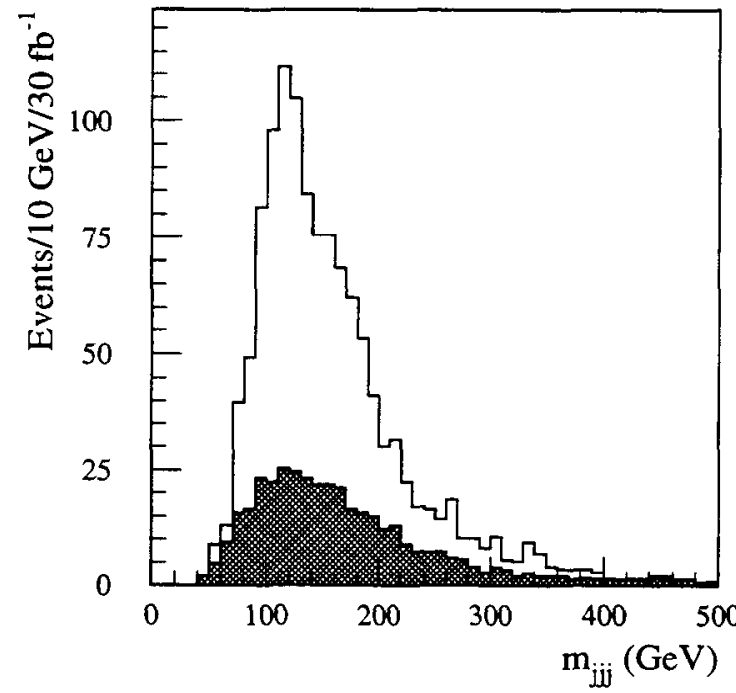

Figure 20-89 Distribution of the invariant mass for all 3-jet combinations passing the selection cuts described in the text for an integrated luminosity of $30 \mathrm{fb}^{-1}$. The cross-hatched distribution shows the SM background. The nominal value of $m_{\tilde{\chi}_{1}^{0}}$ is 122 $\mathrm{GeV}$.

Pairs of three-jet combinations were then built from the jets in the event excluding the two leading ones. A series of cuts on the angular distance and on the transverse momentum of the jets was then applied, reducing the average number of combinations per event to 4.6. A given pairing of the six jets, $j_{1} j_{2} j_{3}$ and $j_{4} j_{5} j_{6}$, in order to be accepted was required to fulfil:

$$
\Delta m_{j j j}=\left|m_{j_{1} j_{2} j_{3}}-m_{j_{4} j_{5} j_{6}}\right|<20 \mathrm{GeV} .
$$

In case more than one combination satisfied the requirement, the one with minimum $\Delta m_{j j j}$ was retained. The resulting mass distribution is shown in Figure 20-89 superimposed to the QCD background for an integrated luminosity of $30 \mathrm{fb}^{-1}$. A very broad peak can be seen at the position corresponding to the $\tilde{\chi}_{1}^{0}$ mass, which corresponds also to the maximum of the QCD background. By using the Monte Carlo information, an efficiency of approximately $80 \%$ was found for reconstructing at least one $\tilde{\chi}_{1}^{0}$ correctly, and $25 \%$ for both.

In the second analysis, all unique pairs of masses passing the above cut were histogrammed, and the combinatorial background under the peak was estimated from the mass of all the neutralino candidates for which

$$
\Delta m_{j j j}>20 \mathrm{GeV}
$$

The upper plots in Figure 20-90 show the distribution of all neutralino candidates, and those in background sample, which is normalised in the region away from the peak, and the signal after background subtraction, for Point 5. The resulting signal peak has a fitted mean of $113 \mathrm{GeV}$, with a width of $15 \mathrm{GeV}, 9 \mathrm{GeV}$ below the true neutralino mass. This shift is due to the loss of energy from the cone used to define the jets, which is estimated in Section 9.1 .2 to be approximately $10 \%$ at low $p_{\mathrm{T}}$. Further work is in progress to optimise the mass estimate and resolution. 

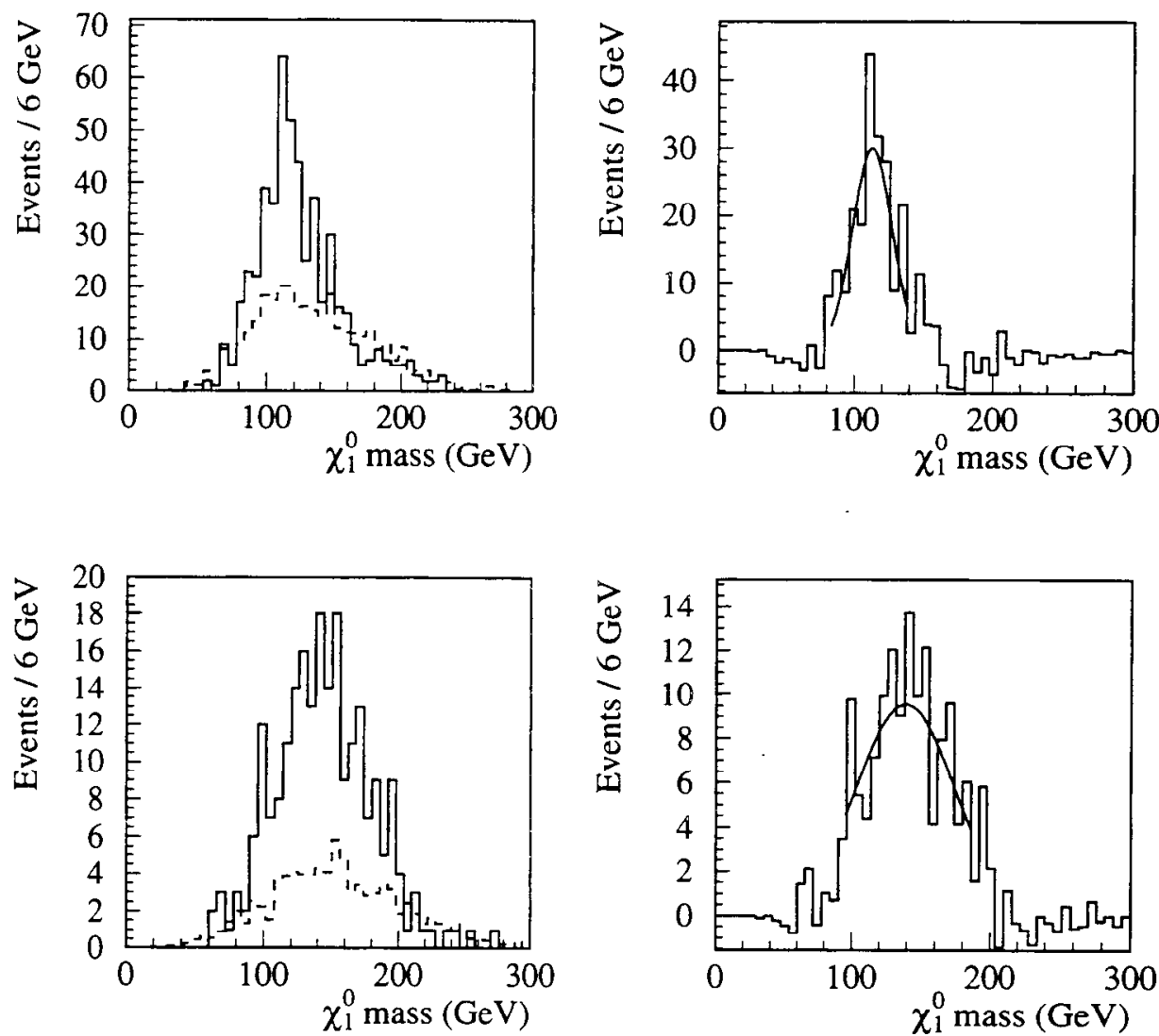

Figure 20-90 Distribution of three-jet combinations invariant masses obtained for $m_{\tilde{\chi}^{0}}=122 \mathrm{GeV}$ (upper plots) $m_{\tilde{\chi}_{0}^{0}}=168 \mathrm{GeV}$ (lower plots). The left hand plots show the $\tilde{\chi}_{1}^{0}$ candidates (solid histogram) and the combinatorial background (dashed histogram), while the left hand plots show the signal after background subtraction. The superimposed fit is to guide the eye to the peak.

The sensitivity of the peak position to the $\tilde{\chi}_{1}^{0}$ mass was studied generating a second event sample for SUGRA Point 1 with a $\tilde{\chi}_{1}^{0}$ mass of $168 \mathrm{GeV}$. The $m_{j j j}$ distributions for this point, before and after background subtraction, are shown in Figure 20-90. The statistical precision is less as the cross-section is smaller. The fitted mass is ( $140 \pm 4.2$ (stat.)) $\mathrm{GeV}$, compared to $151 \mathrm{GeV}$ expected after energy loss from the jet cones. The shift is due to a small bias introduced by the cuts, which were optimised for Point 5 . A data set with looser cuts is in production to study this effect.

From the fit to the background subtracted mass peak, the $\bar{\chi}_{1}^{0}$ mass is expected to be measured with a statistical error of $\pm 3.1 \mathrm{GeV}$. The systematic uncertainties are similar to the ones studied for the top mass reconstruction in Chapter 18, with the difference that in this case the jet combinatorial is higher, the $W$ mass constraint absent, and the $\tilde{\chi}_{1}^{0}$ is lighter than the top. The large available statistics of top events will allow a detailed study with of the systematic effects inherent in multijet mass reconstruction.

From the reconstructed $\tilde{\chi}_{1}^{0}$, using the decay chain $\tilde{\chi}_{2}^{0} \rightarrow \tilde{l}_{R}^{+} l^{\mp} \rightarrow \tilde{\chi}_{1}^{0} l^{+} l^{-}$, the $\tilde{l}_{R}$ and $\tilde{\chi}_{2}^{0}$ peaks can be reconstructed. Events were selected by requiring:

- two reconstructed $\tilde{\chi}_{1}^{0}$ in a window $20 \mathrm{GeV}$ wide centred on the fitted $\tilde{\chi}_{1}^{0}$ mass; 
- two opposite sign, same flavour leptons with $p_{\mathrm{T}}^{l 1}>15 \mathrm{GeV}, p_{\mathrm{T}}^{l 2}>10 \mathrm{GeV}$, and $m_{l l}<109 \mathrm{GeV}$.

The momenta of the three jets were rescaled using the constraint of the measured $\tilde{\chi}_{1}^{0}$ mass.

Since the events used for the $\tilde{\chi}_{1}^{0}$ mass reconstruction were explicitly selected to enhance the contribution of $\tilde{q}_{R} \tilde{q}_{L}$ production, the hardest $\tilde{\chi}_{1}^{0}$ was assumed to come from the decay $q_{R} \rightarrow \tilde{\chi}_{1}^{0} q$. Therefore, the $\tilde{l}_{R}$ mass was reconstructed by calculating the invariant mass of the softer $\tilde{\chi}_{1}^{0}$ with both leptons in the event. The distribution of the lower of these two masses is shown in Figure 20-91, for an integrated luminosity of $30 \mathrm{fb}^{-1}$. The statistics are rather low as the plot includes 119 events, but a clear peak at the nominal $\bar{l}_{R}$ mass $(157 \mathrm{GeV})$ is visible. The statistical uncertainty on the slepton mass was estimated to be $\pm 5.1 \mathrm{GeV}$ by comparing the observed peak with the peaks obtained varying the slepton mass by $\pm 10 \mathrm{GeV}$.

The $\tilde{\chi}_{2}^{0}$ is reconstructed by combining both sleptons with the softer $\tilde{\chi}_{1}^{0}$, after applying a cut around the $\tilde{l}_{R}$ peak. The resulting distribution (Figure 20-92) shows a peak at the nominal $\tilde{\chi}_{2}^{0}$ mass of $233 \mathrm{GeV}$. Within the statistical uncertainty, the peak position follows the $\bar{\chi}_{2}^{0}$ mass, as
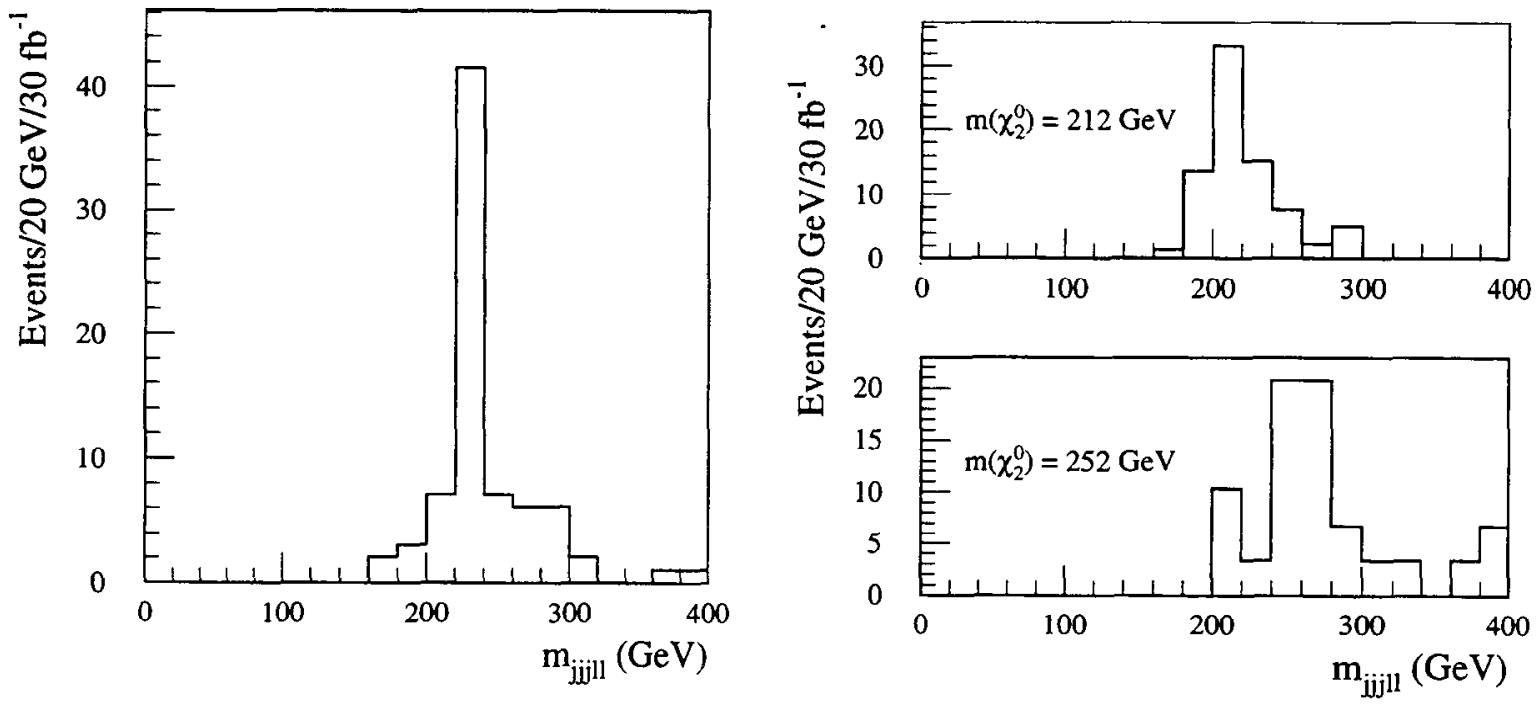

Figure 20-92 Distribution of the invariant mass of the softer $\tilde{\chi}_{1}^{0}$ with the two leptons in the event. The distribution is peaked around the nominal $\tilde{\chi}_{2}^{0}$ mass of $233 \mathrm{GeV}$.

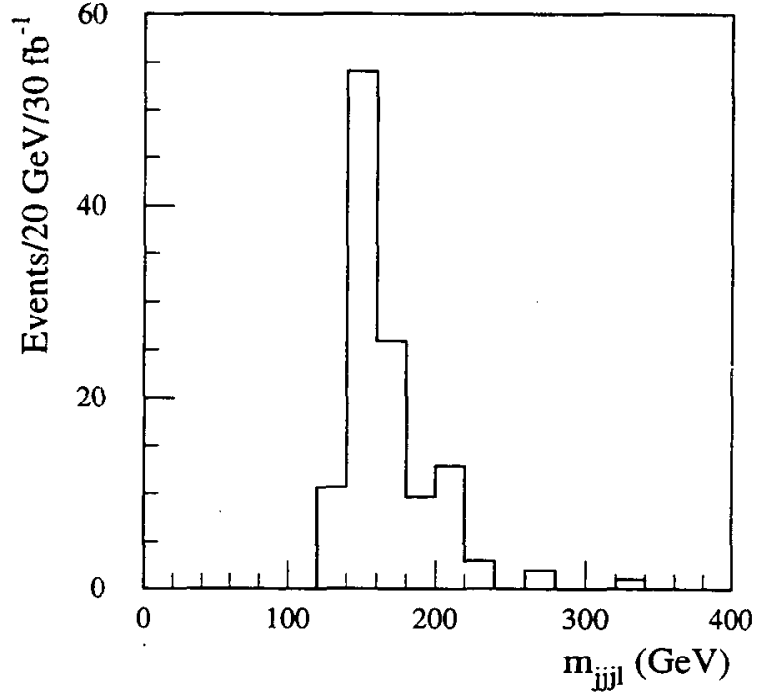

Figure 20-91 Distribution of the smaller invariant mass of the softer $\tilde{\chi}_{1}^{0}$ candidate with a lepton in the event. The distribution is peaked around the nominal $l_{R}$ mass of $157 \mathrm{GeV}$.
Figure 20-93 Distributions of the invariant mass off the softer $\tilde{\chi}_{1}^{0}$ with the two leptons in the event for two different values of the $\tilde{\chi}_{2}^{0}$ mass: $212 \mathrm{GeV}$ (top plot) and $252 \mathrm{GeV}$ (bottom plot). 
shown in Figure 20-93, where the distributions for $\tilde{\chi}_{2}^{0}$ masses varied by $\pm 20 \mathrm{GeV}$ with respect to the nominal one are shown (top and bottom plot). From these distributions, the $\tilde{\chi}_{2}^{0}$ mass was measured to be ( $233 \pm 4.1$ (stat.) \pm 2.3 (syst.) ) GeV .

This same event sample can in principle be used for the direct reconstruction of both $\tilde{q}_{R}$ and $\tilde{q}_{L}$, by combining the hard jets in the event with the reconstructed $\tilde{\chi}_{1}^{0}$ and $\tilde{\chi}_{2}^{0}$ respectively. In practice, the number of fully reconstructed $\tilde{\chi}_{2}^{0}$ is too small to allow a meaningful $\tilde{q}_{L}$ reconstruction to be made. Therefore the analysis was focused on the reconstruction of the decay $q_{R} \rightarrow \bar{\chi}_{1}^{0} q$. The events were selected by requiring two reconstructed $\tilde{\chi}_{1}^{0}$ within $30 \mathrm{GeV}$ of the measured $\tilde{\chi}_{1}^{0}$ mass, and a $p_{T}$ of the leading jet larger than $300 \mathrm{GeV}$, in order to further enhance the fraction of $\tilde{q}_{R}$ decays in the sample. After these cuts $50 \%$ of the events contain a $\tilde{q}_{R}$.

The choice of the correct jet- $\tilde{\chi}_{1}^{0}$ combination was performed in two steps. First each of the two leading jets was combined with the softer $\tilde{\chi}_{1}^{0}$ and the leptons in the event, and the combination giving the lower mass was retained as the $\bar{q}_{L}$. The remaining hard jet was then combined with the harder $\tilde{\chi}_{1}^{0}$. The invariant mass spectrum, shown in Figure 20-94, has a broad peak around $660 \mathrm{GeV}$, the nominal $\tilde{q}_{R}$ mass. By varying the $\bar{q}_{R}$ mass, it was verified that the peak position does follow the $\tilde{q}_{R}$ mass, while it is insensitive to the value of the $\tilde{q}_{L}$ mass. From the invariant mass distribution, the squark mass is measured to be $(664 \pm 30$ (stat.) \pm 7 (syst.) ) $\mathrm{GeV}$.

The reconstruction of the $h \rightarrow b \bar{b}$ peak was straightforward at Point 1 and Point 5 for $R$ parity conserving models. In that case the Standard Model background was efficiently rejected by the $E_{\mathrm{T}}^{\text {miss }}$ requirement. In the $B$-violating case, the $E_{\mathrm{T}}^{\text {miss }}$ cannot be used, and the combined requirement of one lepton and two $b$-jets causes an unacceptable loss of statistics. Moreover, for the considered $\tilde{\chi}_{1}^{0}$ decay, which involves a $c$-quark, the SUSY background would also be big for realistic values of the probability to misidentify a $c$-jet for a $b$-jet. An explicit attempt at reconstruction shows that at Point 5 it is not possible to extract a significant $h \rightarrow b \bar{b}$ peak.

The SUSY events with abundant production of Higgs bosons in $\bar{\chi}_{2}^{0}$ decays would anyway contain a large number of $b$-quarks from $h$ decay, and the observed $b$-jet rate can be used to constrain the sign of $\mu$.

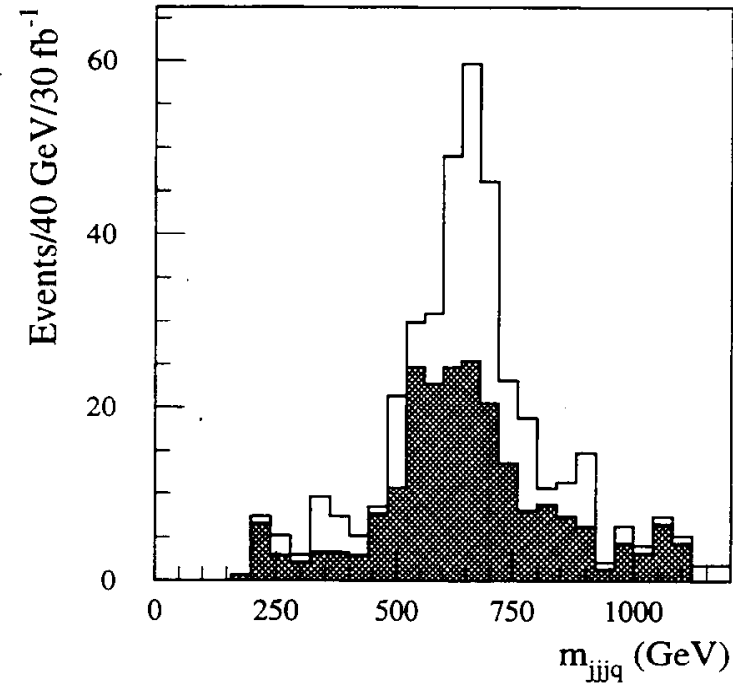

Figure 20-94 Invariant mass of the harder $\tilde{\chi}_{1}^{0}$ with the leading jet in the event. The cross-hatched distribution is for events which do not contain a $\tilde{q}_{R}$. The nominal $\tilde{q}_{R}$ mass is $660 \mathrm{GeV}$.

All the analyses described above are valid only for SUGRA Point 5 . The reconstruction of $\tilde{\chi}_{1}^{0}$ was attempted also for the other SUGRA points. At Points 1 and 2, the sparticles are heavier, so the production cross-section is lower. As shown above, applying the same reconstruction method as for Point 5 gave a peak in the three-jet invariant mass for the signal. The QCD background is $30 \%$ higher than the signal, thus rendering the detection of the three-jet peak more difficult. On the other hand, the higher energy of the jets from the $\tilde{\chi}_{1}^{0}$ decay as compared to Point 5 enhances the fraction of events in which the three jets can be reconstructed. A more detailed study based on the optimisation of the cuts for Points 1 and 2 is needed to assess the achievable precision of the $\tilde{\chi}_{1}^{0}$ mass measurement. At Point 3 , the high production cross-section guarantees the discovery of SUSY, but the low $\bar{\chi}_{1}^{0}$ mass $(44 \mathrm{GeV})$ renders the direct reconstruction of the $\tilde{\chi}_{1}^{0}$ 
from hadronic jets impossible. At Point 4, the mass of the $\bar{\chi}_{1}^{0}$ is $80 \mathrm{GeV}$, and the most promising channel is the electroweak production of chargino-neutralino pairs, which has a cross-section of $3.5 \mathrm{pb}$, followed by purely leptonic decays of the two gauginos, which gives final states with no jets from sparticle decays, except the ones from the two $\bar{\chi}_{1}^{0}$. A detailed study is in progress to verify if the $\tilde{\chi}_{1}^{0}$ can be reconstructed in this case.

\subsubsection{Constraints on the SUGRA parameters}

The basic difference with respect to $R$-parity conserving SUSY is the possibility of direct mass measurements, albeit with very low statistics and limited precision. The studies performed up to now were based on a naive use of the standard jet algorithms. With these algorithms, a convincing reconstruction of the $\tilde{\chi}_{1}^{0}$, and subsequently of the particles decaying into it, seems possible only in the cases where the SUSY mass scale is low enough to guarantee a high production cross-section, and the $\tilde{\chi}_{1}^{0}$ is heavy enough to decay into an identifiable three-jet configuration. These conclusions could be modified by more detailed studies of jet reconstruction. On the other hand, the much increased jet multiplicity renders more difficult the extraction of signatures such as the $h \rightarrow b \bar{b}$ peak from the $\tilde{\chi}_{2}^{0}$ decay. Therefore the value of $\tan \beta$, which is constrained by the Higgs mass measurement, would be accessible only if the direct production of the Higgs boson, e.g. in the $h \rightarrow \gamma \gamma$ channel, could be performed.

In conclusion, for B-violating SUGRA at Point 5, which was studied in detail, the parameters of the model should be constrained with a precision approximately equivalent, or somewhat better, to the one found in the $R$-conserving case except for $\tan \beta$, which requires a measurement outside SUSY final states.

\subsubsection{Lepton number violation: $\chi_{1}{ }^{0} \rightarrow \mu+r v$}

For this class of models, both $\tilde{\chi}_{1}^{0}$ in each event decay into three leptons, of which at least two are of different flavours since the $\lambda_{i j k}$ couplings are antisymmetric. One lepton is neutral, and the other two have opposite charges, since the LSP is supposed to be neutral. The two charged leptons can either have the same flavour or different flavours. The relative weight of the two configurations is a function of the neutralino mixing matrix. For the studies presented here [20-43], the $\tilde{\chi}_{1}^{0}$ decay branching fractions are implemented in the simulation using the program described in [20-44]. This results in a dramatic increase in the number of leptons in the final state, and in the presence of a certain amount of $E_{\mathrm{T}}^{\text {miss }}$. The standard model background is exceedingly small. In case one of the three subscripts of the $\lambda_{i j k}$ takes the value three, one of the leptons is of the third generation, thus reducing the number of 'stable' leptons in the final state. In particular, if the $k$ subscript takes the value three, all the events contain two $\tau$ 's. In these cases the electron and muon multiplicity is significantly reduced, and there is a moderate increase of the $E_{\mathrm{T}}^{\text {miss }}$.

\subsubsection{Inclusive signatures}

The high number of leptons in the final state renders the extraction of the SUSY signal from the Standard Model background very easy. The following selection criteria are sufficient to reduce the Standard Model background to a negligible level:

- at least three leptons with $p_{\mathrm{T}}>20 \mathrm{GeV}$ (lepton = e, $\mu$ ); 
- $E_{\mathrm{T}}^{\mathrm{miss}}>100 \mathrm{GeV}$;

- $p_{\mathrm{T}}>70 \mathrm{GeV}$ for the leading lepton.

In order to study the reach of these selection criteria in the SUGRA parameter space, 1000 events were generated for each point in the $m_{0}-m_{1 / 2}$ plane. The remaining parameters of the models were set to the values: $\tan \beta=2$, $A=0, \mu$ positive. The reach is conventionally expressed as the region in parameter space for which $S / \sqrt{B}>5$, with $S$ number of signal events and $B$ number of background events after the cuts. The reach in the $m_{0}-m_{1 / 2}$ plane for an integrated luminosity of $10 \mathrm{fb}^{-1}$ is shown in Figure 20-95. The black squares represent the points for which $S / \sqrt{B}>5$, and the white squares the points for which, due to the low statistics generated, the sensitivity could not be calculated.

The mass scale of the SUSY events can be studied using a variable similar to the $M_{\text {eff }}$ variable defined for the $R$-parity conserving case, modified to take into account the $\tilde{\chi}_{1}^{0} \mathrm{de}$ cay products:

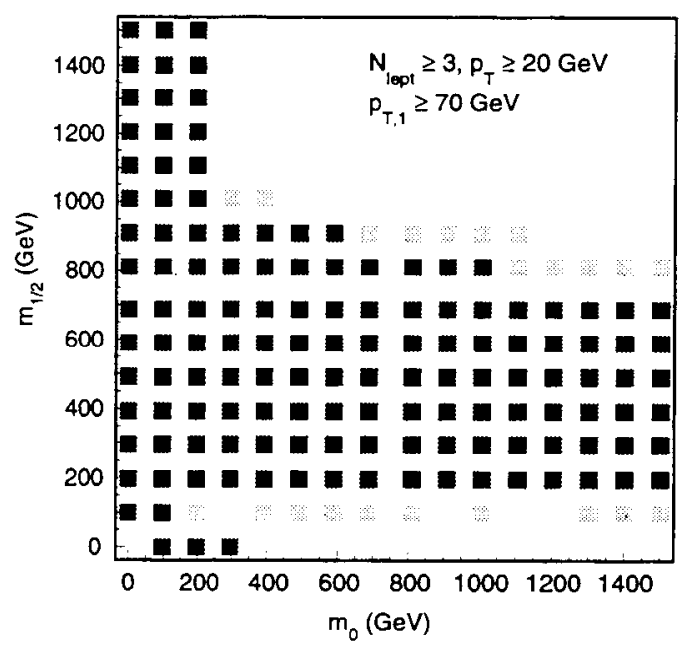

Figure 20-95 Reach in the $m_{0}-m_{1 / 2}$ plane for a model with $\lambda_{123}=10^{-3}$ for an integrated luminosity of $10 \mathrm{fb}^{-1}$.

$$
M_{\mathrm{eff}}=\sum_{i=1}^{4} p_{\mathrm{T}, i}^{j e t}+\sum_{i=1}^{4} p_{\mathrm{T}, i}^{\text {lept }}+E_{\mathrm{T}}^{\mathrm{miss}}
$$

10000 events were generated at 30 random points in the parameter space. For each of these points the correlation between the maximum of the $M_{\text {eff }}$ distribution and $M_{\text {SUGRA }}$ is shown in Figure 20-96, where $M_{\text {SUGRA }}$ is defined as.

$M_{\text {SUGRA }}=\min \left(M_{\bar{g}}, M_{\tilde{q}_{R}}, M_{\tilde{b}_{1}}, M_{\tilde{t}_{1}}\right)$

A good correlation is observed, with a ratio of approximately two.

A fundamental parameter for $R$-parity violating models is the strength of the different couplings $\lambda_{i j k}$. For the decay $\tilde{\chi}_{1}^{0} \rightarrow l^{+} l^{-} v$ the study of the lepton universality violation in the final state should give a hint on which coupling is dominant. To illustrate this, a study was performed for SUGRA Points 1, 3 and 5 and for two dominant couplings: $\lambda_{122}$ and $\lambda_{123}$. For all three points, the number of events with zero electrons and four muons was compared

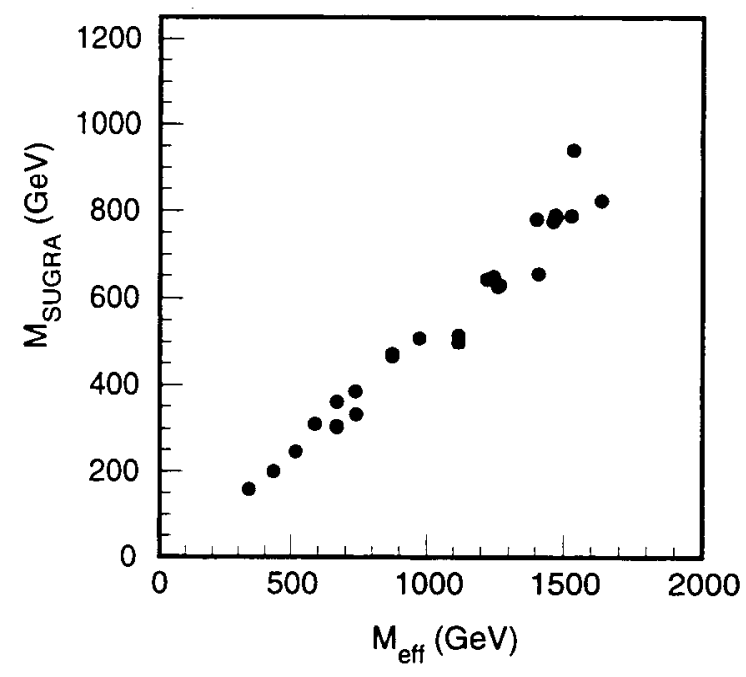

Figure 20-96 Correlation of the maximum of the $M_{\text {eff }}$ distribution and $M_{\text {SUGRA }}$ for 30 points in the SUGRA parameter space. 

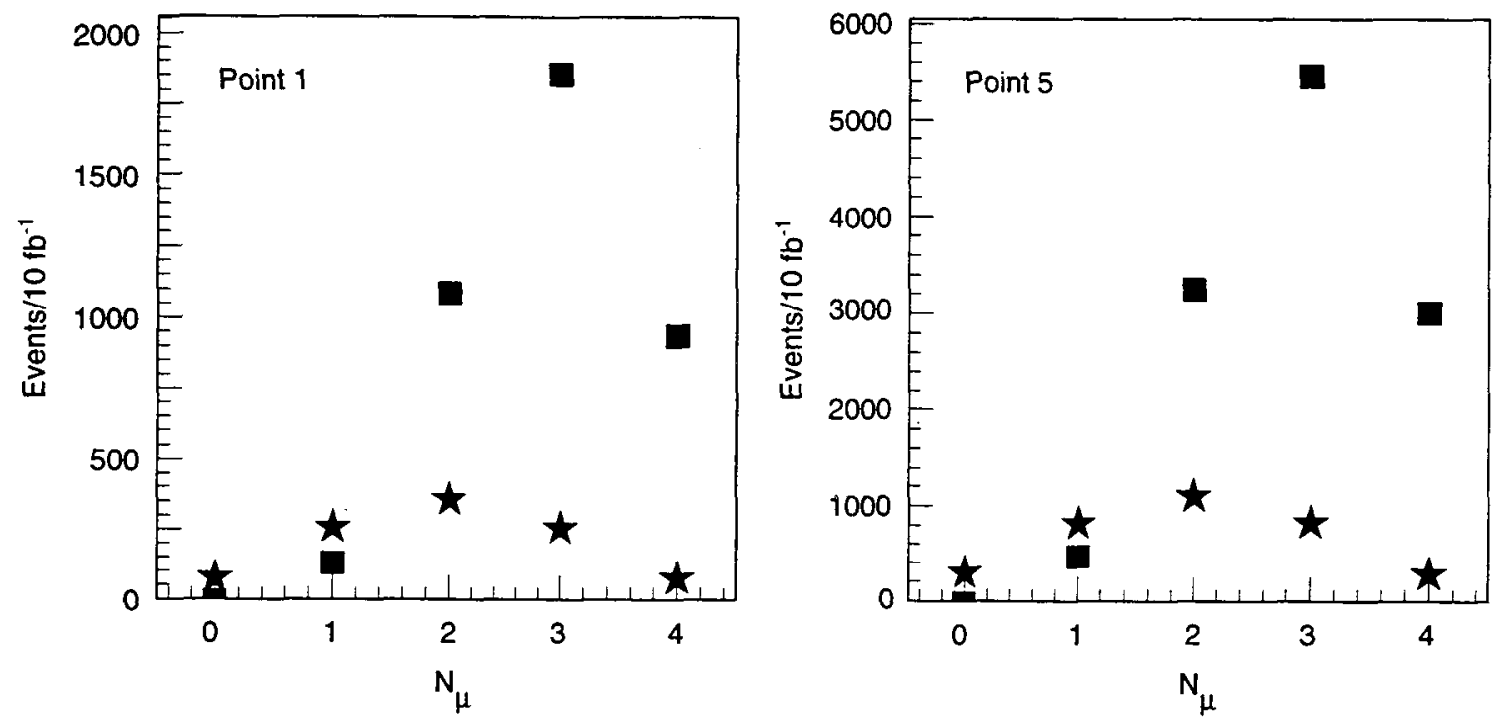

Figure 20-97 Distributions of the number of muons per event if the sum of the number of electrons and muons is equal to four, for two different active couplings for SUGRA Points 1 , and 5 . The full boxes are for $\lambda_{122} \neq 0$ and the stars for $\lambda_{123} \neq 0$.

to the number of events containing four electrons and zero muons. For $\lambda_{122} \neq 0$ the four $-\mu$ events dominate the four-electron events by roughly a factor of ten, whereas for $\lambda_{123} \neq 0$ the two topologies have approximately the same weight, and the overall statistics of four-lepton events is a factor between two and five lower than in the previous case. This is as expected from the index three in the coupling, which produces the decay of the $\tilde{\chi}_{1}^{0}$ into $\tau$ leptons. The pattern can be observed in Figure 20-97 where the number of muons per event is plotted for Points 1 and 5 for two dominant couplings. Only the events for which the sum of the number of electrons and muons is equal to four enter the plot.

\subsubsection{Precision measurements}

The study of detailed signatures was performed for three SUGRA Points: 1, 5, and 3, corresponding respectively to a high, medium and low mass scale. As already discussed in detail in the $R$-parity conserving sections, Points 1 and 5 both have a $h \rightarrow b \bar{b}$ signature. In addition Point 5 has also a lepton-based signature from slepton decay. Point 3 is interesting in this context because the abundant lepton production from $\tilde{\chi}_{2}^{0}$ decays can give a combinatorial background to the $\bar{\chi}_{1}^{0}$ direct reconstruction.

Models with two different dominant $R$-violating couplings were studied: $\lambda_{122} \neq 0$ and $\lambda_{123} \neq 0$. In the first case, each $\tilde{\chi}_{1}^{0}$ decay contains two electrons or muons. For the $\lambda_{123} \neq 0$ coupling, each . $\tilde{\chi}_{1}^{0}$ decay contains a $\tau$ lepton.

\subsubsection{Points 1 and 5: $\lambda_{122} \neq 0$}

The decay of the $\tilde{\chi}_{1}^{0}$ is in this case $\tilde{\chi}_{1}^{0} \rightarrow v_{e(\mu)}+\mu^{ \pm}\left(e^{ \pm}\right)+\mu^{\mp}$. Each event contains at least four leptons. The signature for the $\tilde{\chi}_{1}^{0}$ decay is either a pair of Opposite Sign-Same Flavour leptons (OSSF) or a pair of Opposite Sign-Different Flavour (OS-DF) leptons, with the relative ratio of the two modes determined by the neutralino mixing matrix. 


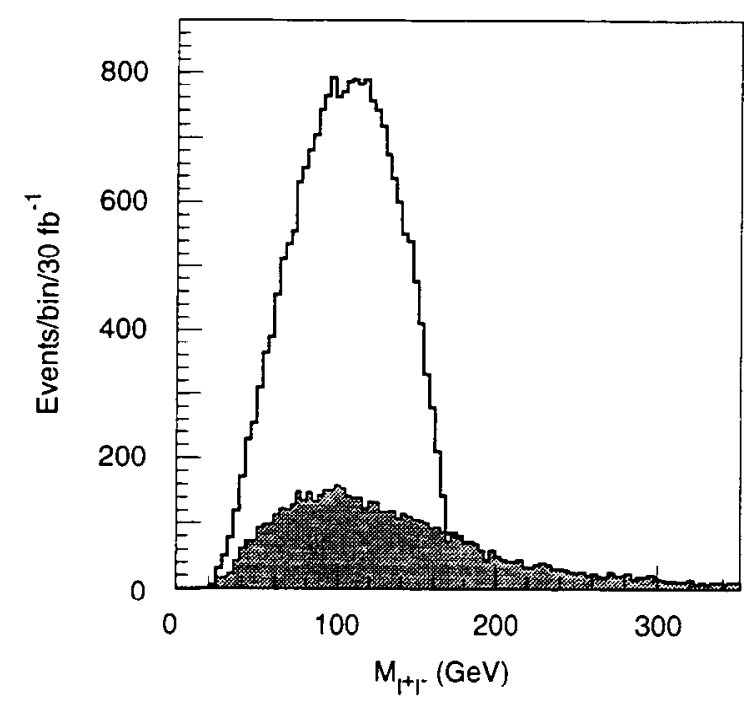

Figure 20-98 Invariant mass distribution for OS-DF lepton pairs at Point 1 , for $\lambda_{122} \neq 0$ and for an integrated luminosity of $30 \mathrm{fb}^{-1}$. The combinatorial background is shown as a shaded histogram.

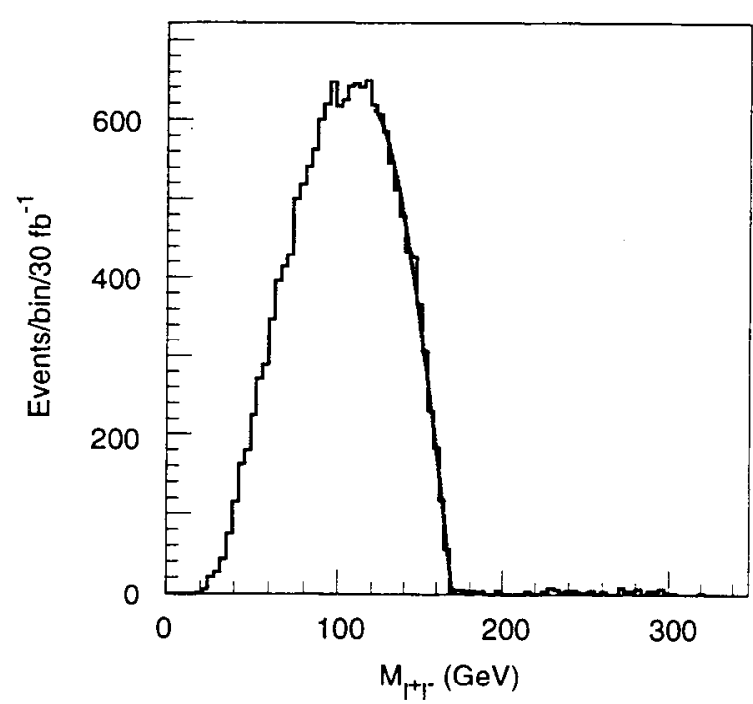

Figure 20-99 Same distribution as for Figure 20-98, after background subtraction. The polynomial fit to the end point is shown.

The events were selected by requiring:

- at least four leptons with $p_{\mathrm{T}}>10 \mathrm{GeV}$;

- $\cos \alpha_{l l} \geq 0.5$, where $\alpha_{l l}$ is the angle between any OS-DF lepton pair;

- $E_{\mathrm{T}}^{\mathrm{miss}}>50 \mathrm{GeV}$.

At Point 1 the production of lepton pairs is dominated by the $\bar{\chi}_{1}^{0}$ decays. At Point 5 there is a significant contribution from the decay $\tilde{\chi}_{2}^{0} \rightarrow \tilde{l}_{R}^{ \pm} l^{\mp} \rightarrow \tilde{\chi}_{1}^{0} l^{+} l^{-}$, which produces OS-SF lepton pairs. Therefore an additional cut: $N_{l}^{+}=N_{i}$ and $N_{e}=N_{\mu}$ was added for Point 5 to reduce the importance of this background.

The distribution of the invariant mass for the OS-DF lepton pairs after the above cuts is shown in Figure 20-98 for Point 1 . There is a clear edge structure superimposed on the combinatorial background. After subtraction of the background parametrised with a Maxwellian function, the $\tilde{\chi}_{1}^{0}$ mass can be measured by fitting the resulting distribution near the end point with a polynomial function, as shown in Figure $20-99$. The resulting values are: $m_{\bar{\chi}_{1}^{0}}=(169.8 \pm 0.18) \mathrm{GeV}$ for Point 1 and $m_{\bar{\chi}_{1}^{0}}=(122.62 \pm 0.35) \mathrm{GeV}$ for Point 5 , where the error is the quadratic sum of the statistical error from the fit to the end point and of the $0.1 \%$ uncertainty in the lepton energy scale.

In the following, a reconstructed $\tilde{\chi}_{1}^{0}$ is defined as an OS-DF lepton pair with an invariant mass in the interval $\left(m_{\tilde{\chi}^{0}}-\Delta m_{\tilde{\chi}_{1}^{0}}, m_{\tilde{\chi}_{1}^{0}}\right)$, where $m_{\tilde{\chi}_{1}^{0}}$ is the measured end point, and $\Delta m_{\tilde{\chi}_{1}^{0}}$ is $50 \mathrm{GeV}$ for
Point 1 and $30 \mathrm{GeV}$ for Point 5 .

To reconstruct the $h \rightarrow b \bar{b}$ peak from the decay $\tilde{\chi}_{2}^{0} \rightarrow h \tilde{\chi}_{1}^{0}$ two additional requirements were applied:

- at least two $b$-jets with $p_{\mathrm{T}}>30(40) \mathrm{GeV}$ for Point 1 (Point 5) and $p_{\mathrm{T}}<300 \mathrm{GeV}$;

- $\cos \alpha_{b b}>0.4(0.3)$ for Point 1 (Point 5), with $\alpha_{b b}$ the angle between the two $b$-jets. 
The invariant mass distribution of the $b \bar{b}$ pairs is shown in Figure 20-100 for Point 1. The background is only from SUSY combinatorial, and it is larger than in the $R$-parity conserving case. This is due to the increase of the background from decays of third-generation squarks, which was suppressed by the lepton veto in the $R$-parity conserving case.

The reconstructed $h$ peak was then combined with a reconstructed $\tilde{\chi}_{1}^{0}$ to form the $\tilde{\chi}_{2}^{0}$ mass. For this purpose the events were required to contain at least one reconstructed $\tilde{\chi}_{1}^{0}$, and a $b \bar{b}$ pair within $\pm 15 \mathrm{GeV}$ of the measured $h$ mass. The angle $\alpha$ between the $h$ and the $\tilde{\chi}_{1}^{0}$ candidates was required to satisfy the condition: $\cos \alpha>0.7(0.5)$ for Point 1 (Point 5).

A harder angular cut was applied at Point 1, as the $\tilde{\chi}_{1}^{0}$ and the $h$ are expected to have a higher boost. The reconstructed $\tilde{\chi}_{2}^{0}$ mass is shown in Figures 20-101 and 20-102 for Points 1 and 5 respectively. From a fit to the peak, the following measurements were obtained: $m_{\tilde{\chi}_{2}^{0}}=(326.2 \pm 4.8) \mathrm{GeV}$ for Point 1 and $m_{\tilde{\chi}_{2}^{0}}=(230.7 \pm 2.7) \mathrm{GeV}$ for Point 5.

The $\tilde{q}_{L}$ has a branching fraction of $60 \%$ into $q \chi_{1}^{+}$for both Points 1 and 5 . The $\chi_{1}^{+}$in turn decays with $100 \%$ branching ratio to $W \tilde{\chi}_{1}^{0}$. This decay can be fully reconstructed by identifying the $q \bar{q}$ decay of the $W$. In events with a reconstructed $\tilde{\chi}_{1}^{0}$, light quark pairs were selected according to the following requirements:

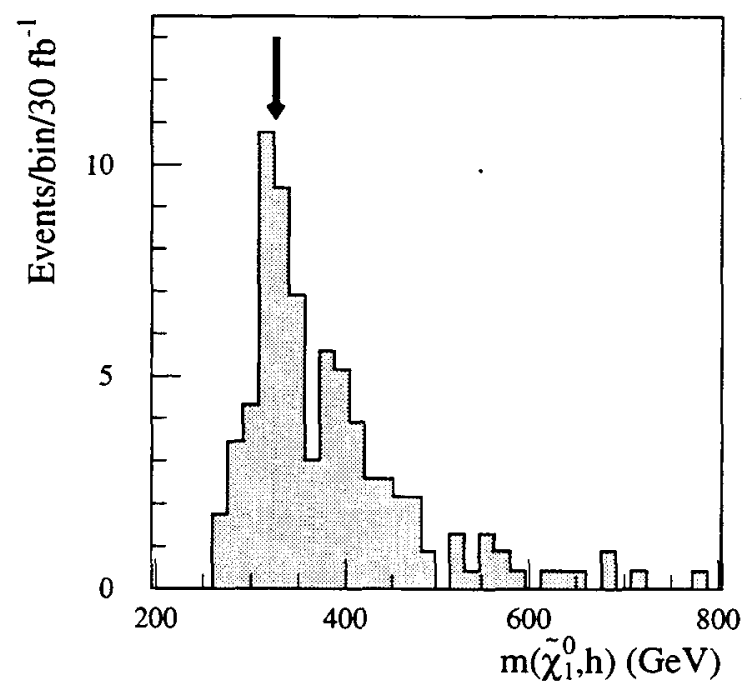

Figure 20-101 Invariant mass of a Higgs candidate with a $\tilde{\chi}_{1}^{0}$ for Point $1\left(\lambda_{122} \neq 0\right)$ and an integrated luminosity of $30 \mathrm{fb}^{-1}$. The distribution shows a peak at the expected position of the $\tilde{\chi}_{2}^{0}$ mass $(326 \mathrm{GeV})$.

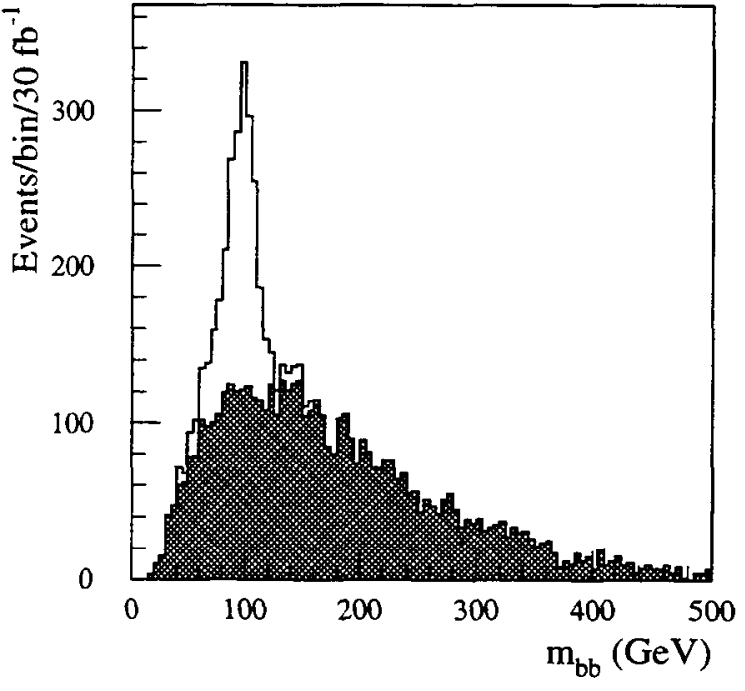

Figure 20-100 Invariant mass of $b \bar{b}$ pairs at Point 1 $\left(\lambda_{122} \neq 0\right)$ for an integrated luminosity of $30 \mathrm{fb}^{-1}$. The full line histogram is the signal+background, the hatched is the SUSY combinatorial background. 


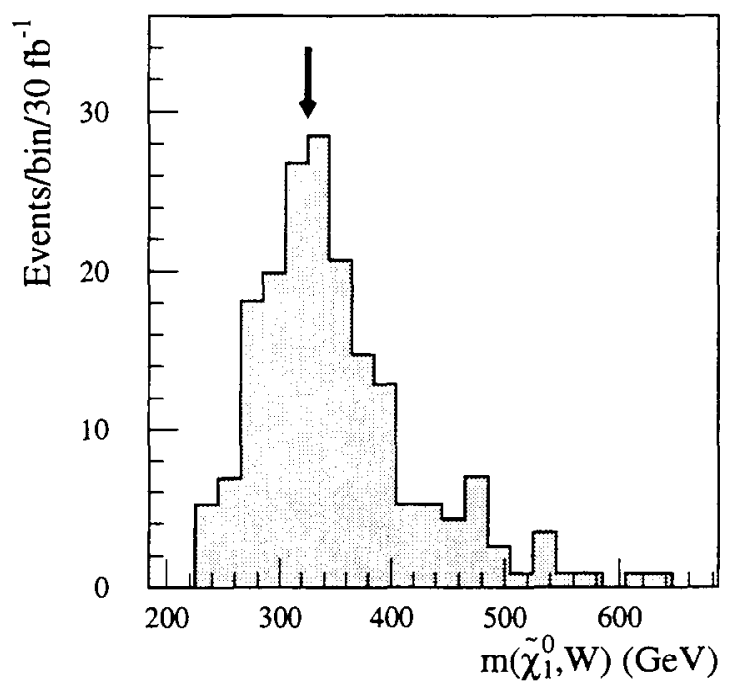

Figure 20-103 Invariant mass of a $W \rightarrow q q$ candidate with a $\tilde{\chi}_{1}^{0}$ candidate for Point $1\left(\lambda_{122} \neq 0\right)$ and an integrated luminosity of $30 \mathrm{fb}^{-1}$. The distribution shows a peak at the expected position of the $\tilde{\chi}_{1}^{+}$mass (326 GeV).

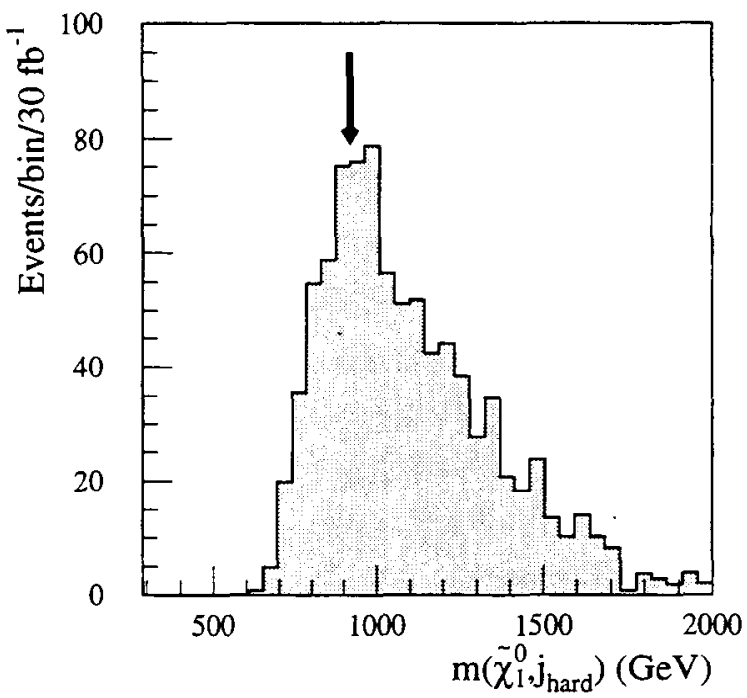

Figure 20-105 Invariant mass of $\tilde{\chi}_{1}^{0}$ with a hard jet in Point $1\left(\lambda_{122} \neq 0\right)$ for an integrated luminosity of $30 \mathrm{fb}^{-1}$. The expected positions of the mass of the right handed squark is shown.

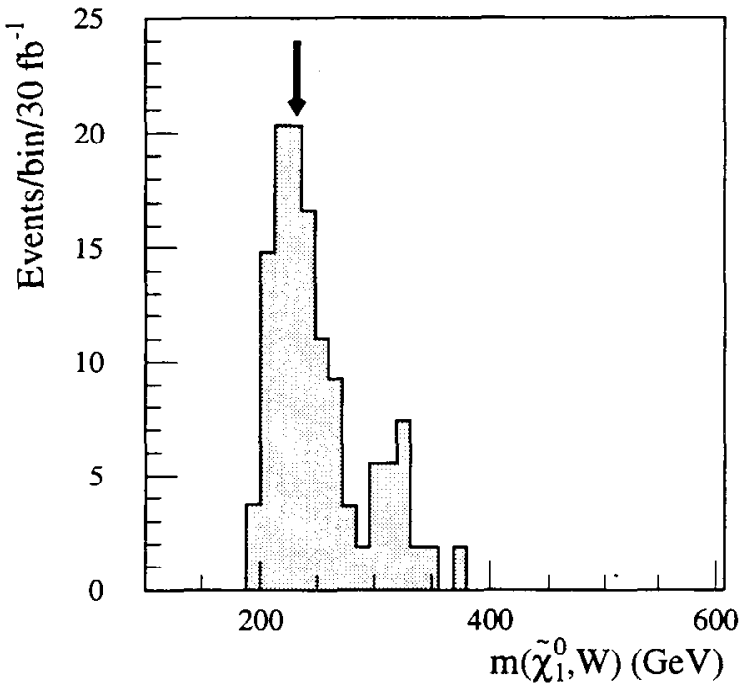

Figure 20-104 Invariant mass of a $W \rightarrow q q$ candidate with a $\tilde{\chi}_{1}^{0}$ candidate for Point $5\left(\lambda_{122} \neq 0\right)$ and an integrated luminosity of $30 \mathrm{fb}^{-1}$. The distribution shows a peak at the expected position of the $\tilde{\chi}_{1}^{+}$mass (232 GeV).

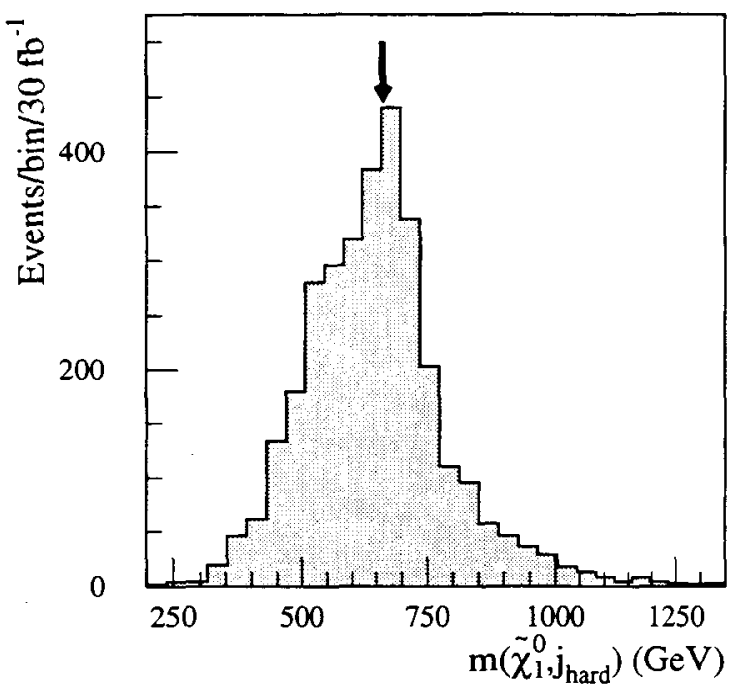

Figure 20-106 Invariant mass of $\tilde{\chi}_{1}^{0}$ with a hard jet in Point $5\left(\lambda_{122} \neq 0\right)$ for an integrated luminosity of $30 \mathrm{fb}^{-1}$. The expected positions of the masses of the right handed squark is shown.

- $p \uparrow^{1}>100 \mathrm{GeV}$ and $p j^{2}<600$ (350) GeV for Point 1 (Point 5);

- $\cos \alpha_{j j}>0.9(0.8)$ for Point 1 (Point 5).

The invariant mass distribution of the two selected jets shows a clear peak around the $W$ mass over a large combinatorial background. The $W$ was selected by requiring an invariant mass within $15 \mathrm{GeV}$ of the nominal $W$ mass. The $W$ candidate was then combined with the $\tilde{\chi}_{1}^{0}$ if the angle $\alpha$ between them satisfied the requirement $\cos \alpha>0.85$. The resulting invariant mass is 


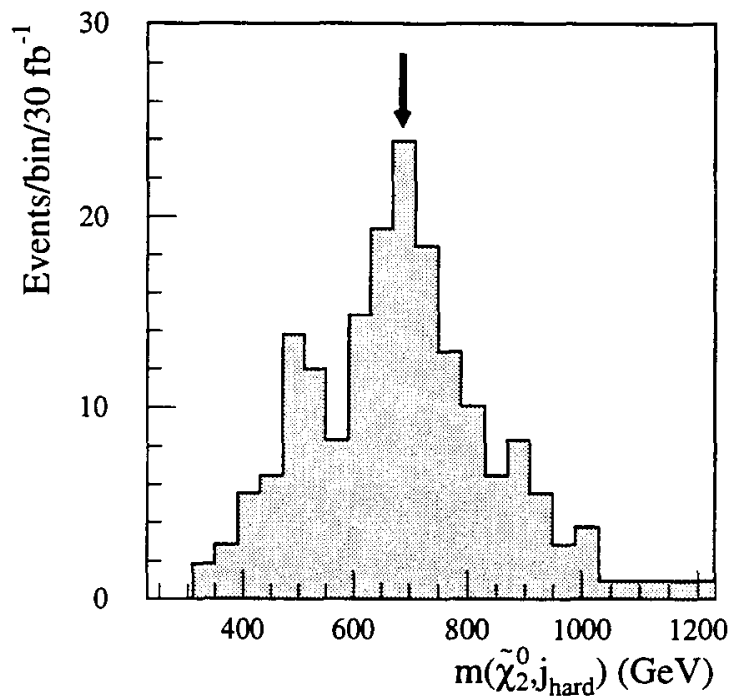

Figure 20-107 Invariant mass of a $\tilde{\chi}_{2}^{0}$ candidate with a hard jet at Point $5\left(\lambda_{122} \neq 0\right)$. The position of the expected peak for the $\tilde{q}_{L}$ is shown.

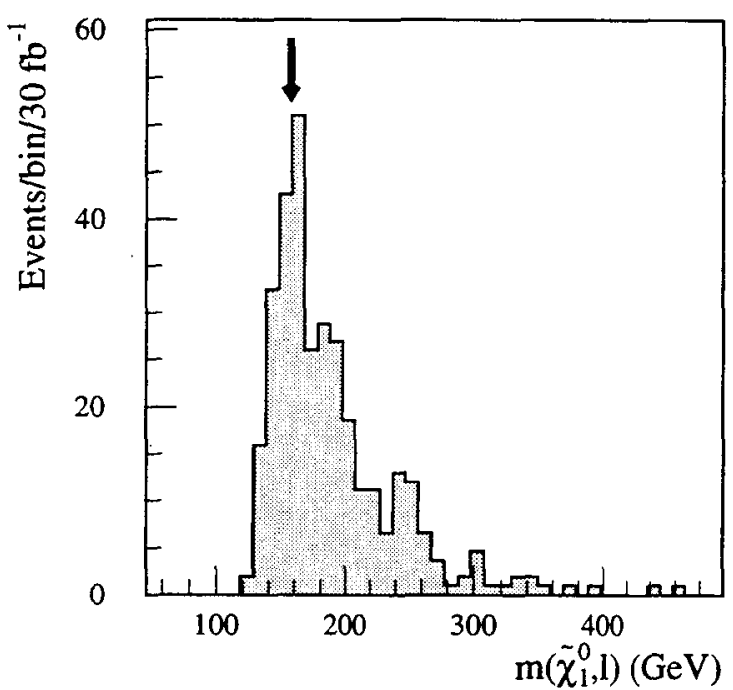

Figure 20-108 Invariant mass of the $\tilde{\chi}_{1}^{0}$ with a lepton at Point $5\left(\lambda_{122} \neq 0\right)$. A peak at the expected position of the $\tilde{l}_{R}$ mass can be seen.

shown in Figures 20-103 and 20-104 for Points 1 and 5 respectively. A peak is visible at the value of the $\chi_{1}^{+}$mass, for both points. Due to the high combinatorial background under the selected $W$, the peak is very broad and the statistics is low. From the peak width and the number of events, an uncertainty on the $\chi_{1}^{+}$mass measurement of around $6 \mathrm{GeV}$ for Point 1 and $4 \mathrm{GeV}$ for Point 5 can be estimated.

Squark reconstruction can be attempted for $\tilde{q}_{R}$ by exploiting the decay $\tilde{q}_{R} \rightarrow q \tilde{\chi}_{1}^{0}$. Events were selected requiring at least one reconstructed $\tilde{\chi}_{1}^{0}$ and a hard jet. The hard jet was required not to give an invariant mass within $15 \mathrm{GeV}$ of the $Z$ or of the $W$ mass combined with any other light jet in the event and to be in the same hemisphere as the $\tilde{\chi}_{1}^{0}$ candidate.

The invariant mass distributions are shown in figures Figures 20-105 and 20-106 for Points 1 and 5 respectively. The observed peaks are very broad, and suffer from contaminations from gluino and $\tilde{t}_{1}$ decays. In particular at Point 1 the $\tilde{q}_{R}$ mass is rather close to the gluino mass, and it is difficult to determine if the observed peak is actually due to $\tilde{q}_{R}$ decay. The situation looks clearer for Point 5 , where the $\tilde{q}_{R}$ peak is clean, and has a good statistical significance. In order to extract a measurement from these distributions a detailed knowledge of the combinatorial background is necessary.

Given the low statistics of reconstructed $\tilde{\chi}_{2}^{0}$ (see Figures 20-101 and 20-102), the decay $\tilde{q}_{L} \rightarrow q \tilde{\chi}_{2}^{0}$ can only be reconstructed for Point 5 . The events were required to include a reconstructed $\tilde{\chi}_{2}^{0}$ within $40 \mathrm{GeV}$ of the measured $\bar{\chi}_{2}^{0}$ peak, and a hard jet with $p_{T}>100 \mathrm{GeV}$ which cannot be ascribed to a $W \rightarrow j j$ decay. The resulting invariant mass distribution is shown in Figure 20-107. The peak at the $\tilde{q}_{L}$ mass is clean and has a reasonable statistics, allowing the $\tilde{q}_{L}$ mass to be measured with a precision of about $15 \mathrm{GeV}$. The distribution presents an enhancement around $500 \mathrm{GeV}$, which, if statistically significant could be interpreted as a $\tilde{t}_{1} \rightarrow \tilde{\chi}_{2}^{0} t \rightarrow \tilde{\chi}_{2}^{0} j j b$ decay where the three jets from the top decay are reconstructed as a single jet.

At Point 5 the right handed sleptons $\tilde{l}_{R}$ are produced in $\tilde{\chi}_{2}^{0}$ decays, and can be reconstructed from their dominant decay $\tilde{l}_{R} \rightarrow \tilde{\chi}_{1}^{0} l$. Events were selected by requiring a $\tilde{\chi}_{1}^{0}$ candidate and an additional lepton. The lepton transverse momentum was required to be between 10 and 
$200 \mathrm{GeV}$, and the angle $\alpha$ between the $\tilde{\chi}_{1}^{0}$ and the lepton to satisfy the condition $\cos \alpha \geq 0.5$. The invariant mass distribution is shown in Figure 20-108. The peak is superimposed on a combinatorial background mainly coming from the decay chain $\bar{\chi}_{1}^{ \pm} \rightarrow \bar{\chi}_{1}^{0} W^{ \pm} \rightarrow \bar{\chi}_{1}^{0} l^{ \pm} v_{l}$. From a Gaussian fit to the peak the slepton mass is measured to be $m_{\tilde{l}_{R}}=(156.8 \pm 1.7) \mathrm{GeV}$.

20.4.2.4 Points 1 and 5: $\lambda_{123} \neq 0$

In this case there is always a $\tau$ lepton among the $\tilde{\chi}_{1}^{0}$ decay product. The clear OS-DF signature exploited for the case $\lambda_{122} \neq 0$ is therefore lost, and the direct reconstruction of the $\tilde{\chi}_{1}^{0}$ is very difficult. The aim of the analysis is in this case to check if the signatures studied in the $R$-parity conserving case are still valid.

At Point 5 the $\tilde{\chi}_{2}^{0}$ decay chain $\tilde{\chi}_{2}^{0} \rightarrow \tilde{l}_{R}^{t} l^{\mp} \rightarrow \tilde{\chi}_{1}^{0} l^{+} l^{-}$with the subsequent $\tilde{\chi}_{1}^{0}$ decay yields three leptons, among which an OS-SF and an OS-DF flavour pair can be formed. The lepton-lepton edge from the decay $\tilde{\chi}_{2}^{0} \rightarrow \tilde{l}_{R}^{ \pm} l^{\mp} \rightarrow \tilde{\chi}_{1}^{0} l^{+} l^{-}$has a big combinatorial background from the additional leptons in the event. The events were selected in the same way as for the $\tilde{\chi}_{1}^{0}$ mass reconstruction, requiring the two OS-SF leptons to have $p_{T}>15 \mathrm{GeV}$, to be in the same hemisphere and to be unbalanced. The resulting invariant mass distribution of OS-SF lepton pairs is shown in Figure 20-109. An edge structure is visible above the combinatorial background. The precision with which this can be measured has not been investigated.

The OS-DF pairs with an invariant mass near the endpoint can be further combined with an OS lepton pair in the event, where one of the additional leptons comes from the $\tilde{\chi}_{1}^{0}$ decay, and the other one from the tau decay. If the mass of the OS-DF pair is rescaled to the value of the measured end point, the invariant mass distribution with the two additional leptons presents an edge which is sensitive the $\tilde{\chi}_{2}^{0}$ mass.

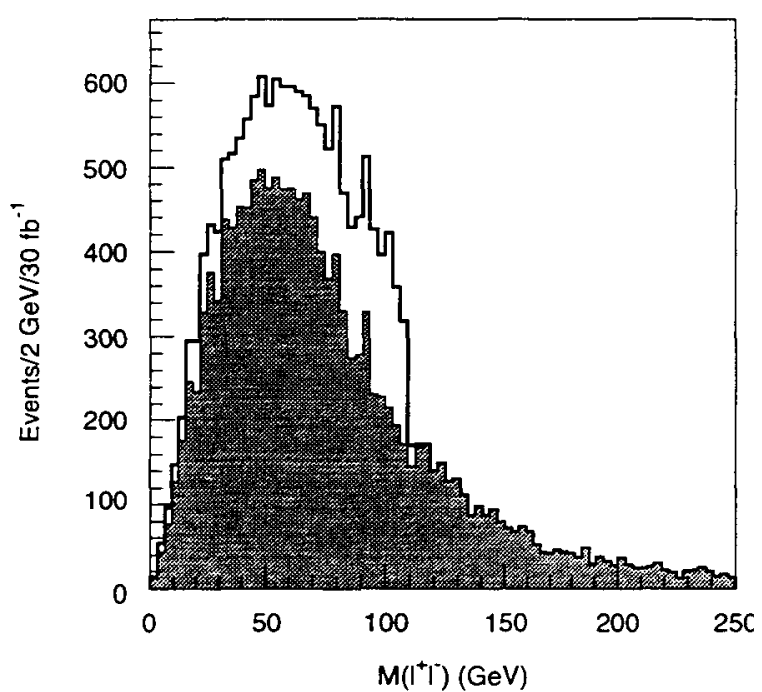

Figure 20-109 Invariant mass of OS-SF lepton pairs fat Point $5\left(\lambda_{123} \neq 0\right)$ for an integrated luminosity of $30 \mathrm{fb}^{-1}$. The full line histogram is signal+background and the shaded histogram the combinatorial background.

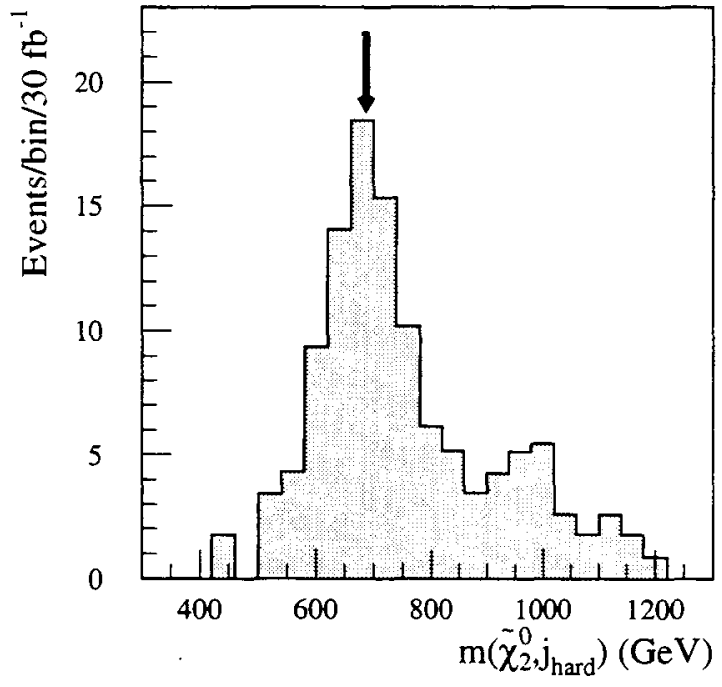

Figure 20-110 invariant mass of the jet- $\tilde{\chi}_{2}^{0}$ combination as described in the text at Point $5\left(\lambda_{123} \neq 0\right)$ for an integrated luminosity of $30 \mathrm{fb}^{-1}$. The arrows shows the expected position of the $\tilde{q}_{L}$ mass. 
By further combining the 4-lepton combinations with an invariant mass near the $\tilde{\chi}_{2}^{0}$ edge with a hard jet in the event, the distribution in Figure $20-110$ is obtained which is peaked at the value of the $\tilde{q}_{L}$ mass.

\subsubsection{Point 3: $\lambda_{122} \neq 0$}

Point 3 has a much lower mass scale than the other points, and therefore a huge production cross-section. The dominant decay of the $\tilde{\chi}_{2}^{0}$ is the three-body decay $\chi_{2}^{0} \rightarrow f \bar{f} \chi_{1}^{0}$, where $f$ are ordinary fermions, yielding a large number of OS-SF lepton pairs in the final state. The $\tilde{\chi}_{1}^{0}$ decays $\tilde{\chi}_{1}^{0} \rightarrow v_{e(\mu)}+\mu^{ \pm}\left(e^{ \pm}\right)+\mu^{\mp}$ yield both OS-DF and OS-SF pairs, with relative branching fractions 'a priori' different from those at point 5 . There is a large violation of electron-muon universality in this case; the consequences of this have not been studied. The $\tilde{\chi}_{1}^{0}$ reconstruction is e performed by calculating the invariant mass of OS-DF pairs which have a lower background. Events were selected requiring at least four leptons with $p_{\mathrm{T}}>10 \mathrm{GeV}$ in the final state, and an $E_{T}^{\text {miss }}$ greater than $50 \mathrm{GeV}$. The angle $\alpha_{l l}$ between the two leptons was required to satisfy the condition $\cos \alpha_{l l} \geq 0.85$.

The invariant mass distribution of the lepton pairs is shown in Figure 20-111 for an integrated luminosity of $10 \mathrm{fb}^{-1}$. The background is higher than in Point 5 , and is mostly produced by the decays of $\tilde{t}_{1}$ and $\tilde{b}_{1}$ to charginos. The statistics is high, and the $\tilde{\chi}_{1}^{0}$ mass can be measured as $m_{\tilde{\chi}_{1}^{0}}=(44.8 \pm 0.1) \mathrm{GeV}$ from a fit to the end point of the distribution after background subtraction. The quoted error includes the statistical error on the fit and a $0.1 \%$ uncertainty on the lepton energy scale. A detailed study on the modelling of the background is still needed in order to confirm that this precision is indeed achievable.

For the following steps in the reconstruction of the SUSY decay chains, a reconstructed $\tilde{\chi}_{1}^{0}$ is defined as an OS-DF lepton pair with invariant mass smaller than and within $10 \mathrm{GeV}$ of the endpoint. The momenta of the two leptons are scaled up to the measured $\tilde{\chi}_{1}^{0}$ mass.

Information on the $\tilde{\chi}_{2}^{0}$ mass can be extracted using additional opposite-sign leptons in events with at least one reconstructed $\tilde{\chi}_{1}^{0}$. The invariant mass of the lepton pairs has a complex structure, with two edges superimposed: one at $45 \mathrm{GeV}$, and one at $42 \mathrm{GeV}$, corresponding to $m_{\tilde{\chi}_{1}^{0}}$ and $m_{\tilde{\chi}_{2}^{0}}-m_{\tilde{\chi}_{1}^{0}}$ respectively. The distribution is shown in Figure 20-112, where the full histogram shows the sum of the $\tilde{\chi}_{1}^{0} \rightarrow l^{+} l^{-} v$ and of the $\tilde{\chi}_{2}^{0} \rightarrow \tilde{\chi}_{1}^{0} l^{+} l$ contributions, and the shaded histogram the distribution of the $\tilde{\chi}_{1}^{0}$ decay alone. Already from this distribution, the $\tilde{\chi}_{1}^{0}-\bar{\chi}_{2}^{0}$ mass difference can be measured with a precision of about $1 \%$, if the background can be adequately subtracted. Alternatively, a full reconstruction of the $\tilde{\chi}_{2}^{0}$ can be performed by calculating the invariant mass of a reconstructed $\tilde{\chi}_{1}^{0}$ with a lepton pair near the $m_{\tilde{\chi}_{2}^{0}}-m_{\tilde{\chi}_{1}^{0}}$ edge. From a gaussian fit to the distribution shown in Figure 20-113, the $\tilde{\chi}_{2}^{0}$ mass is measured as $m_{\tilde{\chi}^{0}}=(96.7 \pm 0.17)$, where the quoted error includes the statistical error on the fit, the statistical error on the $\tilde{\chi}_{1}^{0}$ mass measurement, and the systematic uncertainty on the lepton energy scale.

The most striking feature of Point 3 is the high number of $b$-jets in the final state. The dominant production mechanism is the decay chain $\bar{g} \rightarrow \tilde{b}_{1}+b \rightarrow \tilde{\chi}_{2}^{0}+b+b$. A full reconstruction can be performed by using $\tilde{\chi}_{2}^{0}$ candidates defined as four-lepton combinations with an invariant mass within $\pm 10 \mathrm{GeV}$ of the $\tilde{\chi}_{2}^{0}$ peak shown in Figure $20-113$. The $b$-jets from the decay $\tilde{b}_{1} \rightarrow \bar{\chi}_{2}^{0}+b$ have typically a $p_{T}$ in excess of $50 \mathrm{GeV}$, whereas the $b$-jets from the decay $\tilde{g} \rightarrow \tilde{b}_{1}+b$ are much softer. Therefore events were selected requiring at least two $b$-jets with $p_{\mathrm{T}}>10 \mathrm{GeV}$ among which the ones with $p_{\mathrm{T}}>50 \mathrm{GeV}$ were labelled as 'hard' and the remaining ones as 'soft'. 


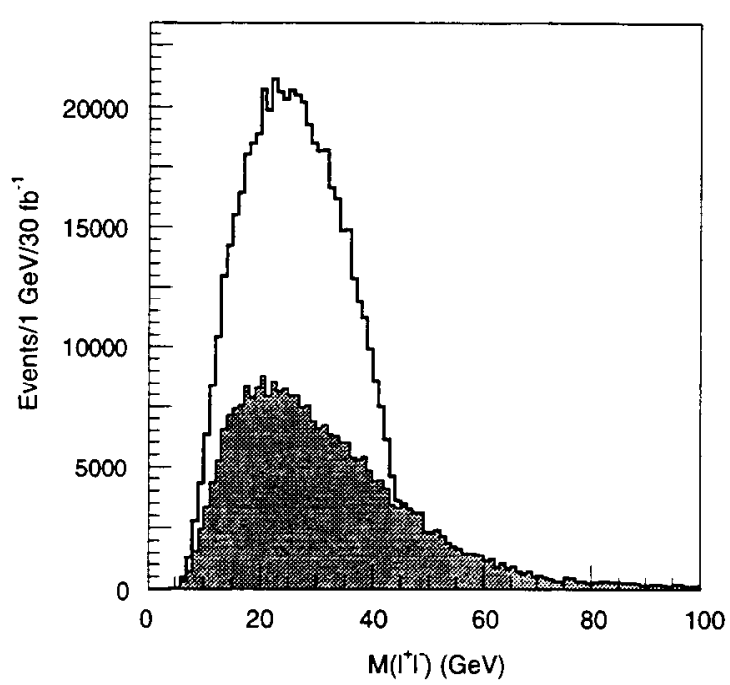

Figure 20-111 Mass of the OS-DF lepton pairs at Point $3\left(\lambda_{122} \neq 0\right)$ for an integrated luminosity of $10 \mathrm{fb}^{-1}$. The full line histogram is the signal+background, the shaded histogram the combinatorial background.

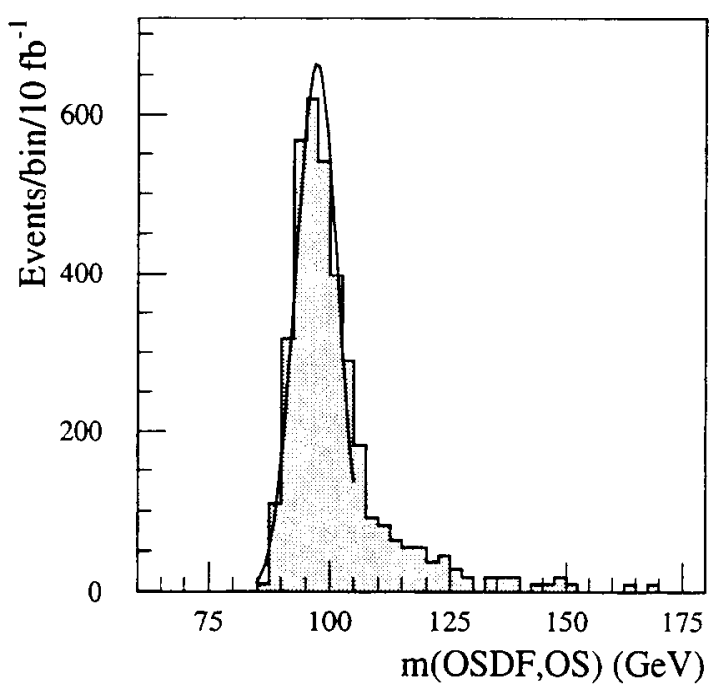

Figure 20-113 Invariant mass distribution of a $\tilde{\chi}_{1}^{0}$ and an OS lepton pair at Point $3\left(\lambda_{122} \neq 0\right)$ for an integrated luminosity of $10 \mathrm{fb}^{-1}$. The fitted peak gives a measurement of the $\tilde{\chi}_{2}^{0}$ mass.

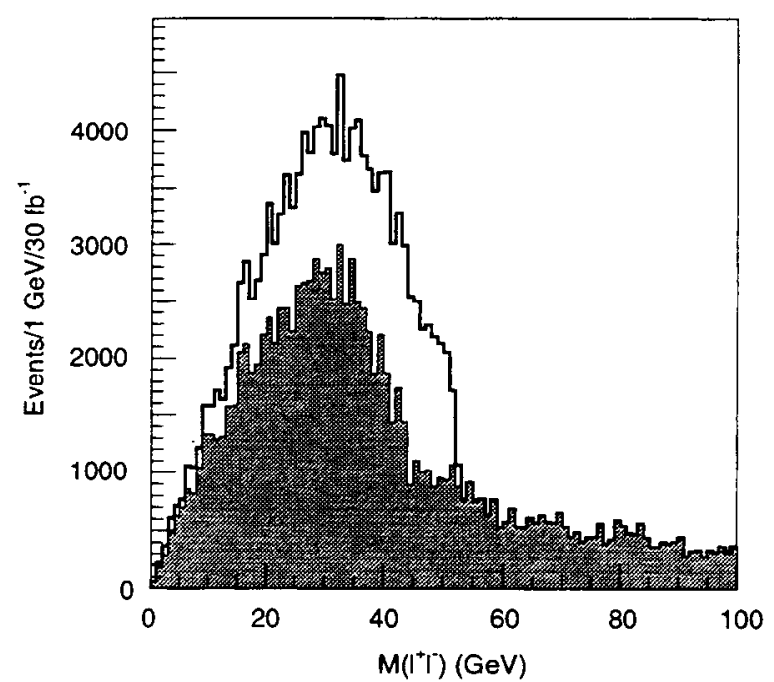

Figure 20-112 Invariant mass of the remaining OS lepton pairs after removal of the $\tilde{\chi}_{1}^{0}$ candidate at Point $3\left(\lambda_{122} \neq 0\right)$ for an integrated luminosity of $10 \mathrm{fb}^{-1}$.

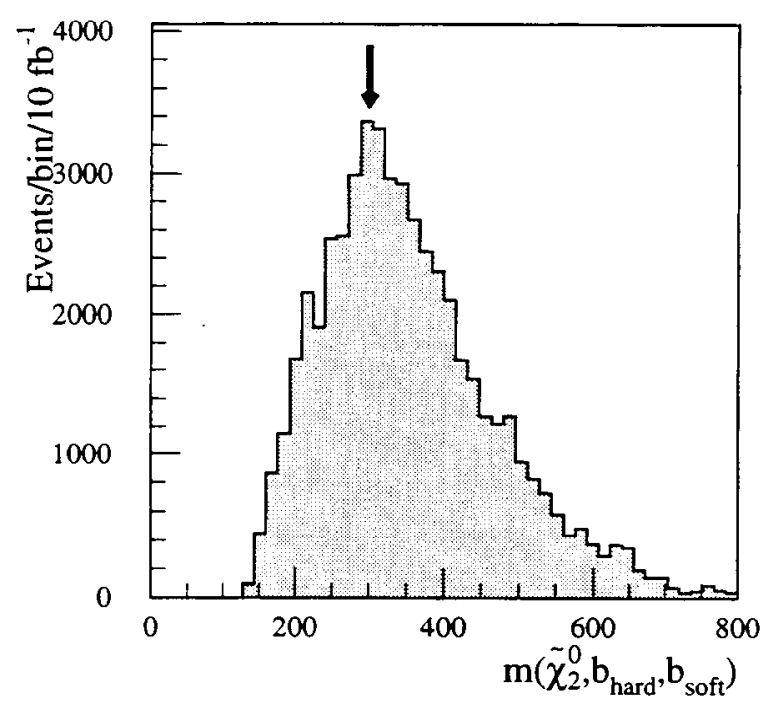

Figure 20-114 Invariant mass of a candidate $\tilde{\chi}_{2}^{0}$ with two $b$-jets at Point $3\left(\lambda_{122} \neq 0\right)$ for an integrated luminosity of $10 \mathrm{fb}^{-1}$. The position of the peak corresponds to the mass of the gluino.

The first step is to reconstruct a gluino peak by taking the invariant mass of a reconstructed $\tilde{\chi}_{2}^{0}$, a 'hard' $b$-jet, and a 'soft' $b$-jet. The distribution, shown in Figure 20-114, exhibits a broad peak around $300 \mathrm{GeV}$, which corresponds to the gluino mass. The events within $\pm 10 \mathrm{GeV}$ of the gluino peak were then selected, and the invariant mass of the $\tilde{\chi}_{2}^{0}$ with the 'hard' $b$ was calculated if the angle $\alpha$ between them satisfied $\cos \alpha \geq 0.5$. The resulting mass distribution is shown in 


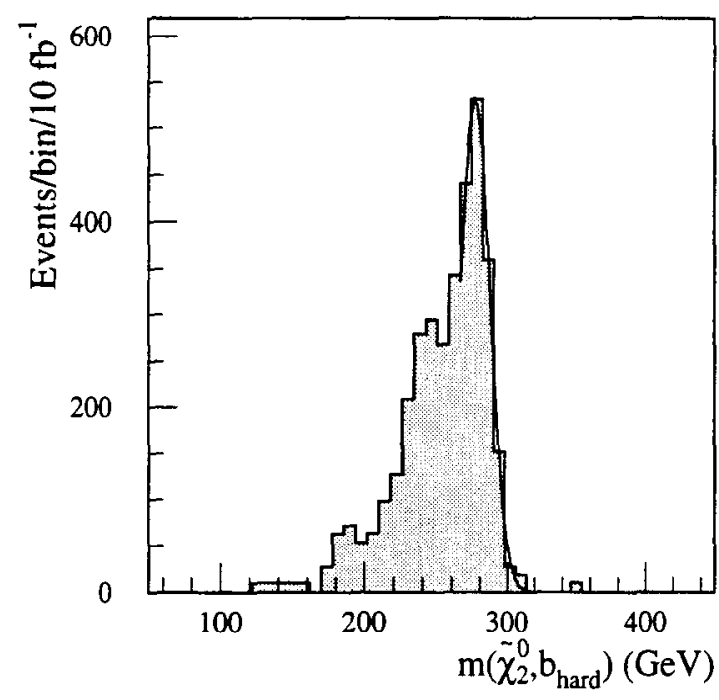

Figure 20-115 Invariant mass of the $\tilde{\chi}_{2}^{0}$ with a hard $b$-jet at Point $3\left(\lambda_{122} \neq 0\right)$ for an integrated luminosity of $10 \mathrm{fb}^{-1}$. The events are selected requiring that the $\bar{\chi}_{2}^{0}-b b$ mass is near the gluino mass.

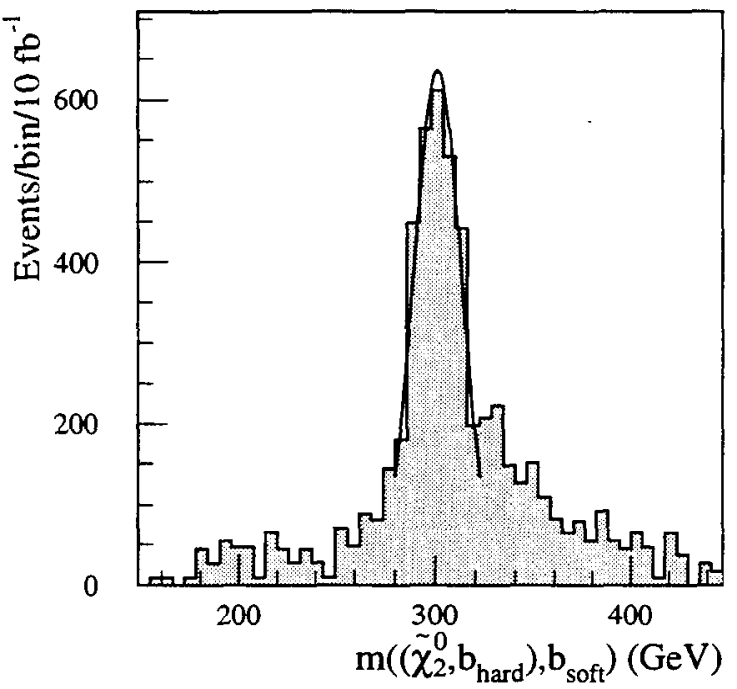

Figure 20-116 Invariant mass of the $\tilde{b}_{1}$ with the remaining soft $b$-jet in the event at Point $3\left(\lambda_{122} \neq 0\right)$ for an integrated luminosity of $10 \mathrm{fb}^{-1}$. The fitted peak gives a measurement of the gluino mass.

Figure 20-115. From a gaussian fit to the observed peak the $\tilde{b}_{1}$ mass is measured to be $m_{\tilde{b}_{1}}=(276.6 \pm 3) \mathrm{GeV}$, where the error is dominated by the uncertainty in the $b$-jet energy scale. For the events within $\pm 10 \mathrm{GeV}$ of the $\tilde{b}_{1}$ peak, the invariant mass of the $\tilde{b}_{1}$ candidate with the soft $b$-jet can be calculated, as shown in Figure 20-116, yielding a measurement of the gluino mass: $m_{\tilde{g}}=(301 \pm 3) \mathrm{GeV}$.

20.4.2.6 Point 3: $\lambda_{123} \neq 0$

The direct reconstruction of $\tilde{\chi}_{1}^{0}$, as in Point 5 , is rendered impossible by the presence of a tau lepton among the $\tilde{\chi}_{1}^{0}$ decay products. The possible measurements are therefore very similar to the $R$-parity conserving case, and will only be briefly summarised in the following. The mass difference $m_{\tilde{\chi}_{2}^{0}}-m_{\bar{\chi}_{1}^{0}}$ can be measured from the end point of the invariant mass distribution of the OS-SF lepton pairs. The reconstruction of the $\tilde{g} \rightarrow \tilde{b}_{1}+b \rightarrow \tilde{\chi}_{2}^{0}+b+b$ decay chain follows the procedure described above for the $\lambda_{122} \neq 0$ case. The main difference is that no direct measurement of the $\tilde{\chi}_{2}^{0}$ mass is available, therefore one needs to assume a value for the $\tilde{\chi}_{2}^{0}$ mass in order to reconstruct gluino and $\tilde{b}_{1}$.

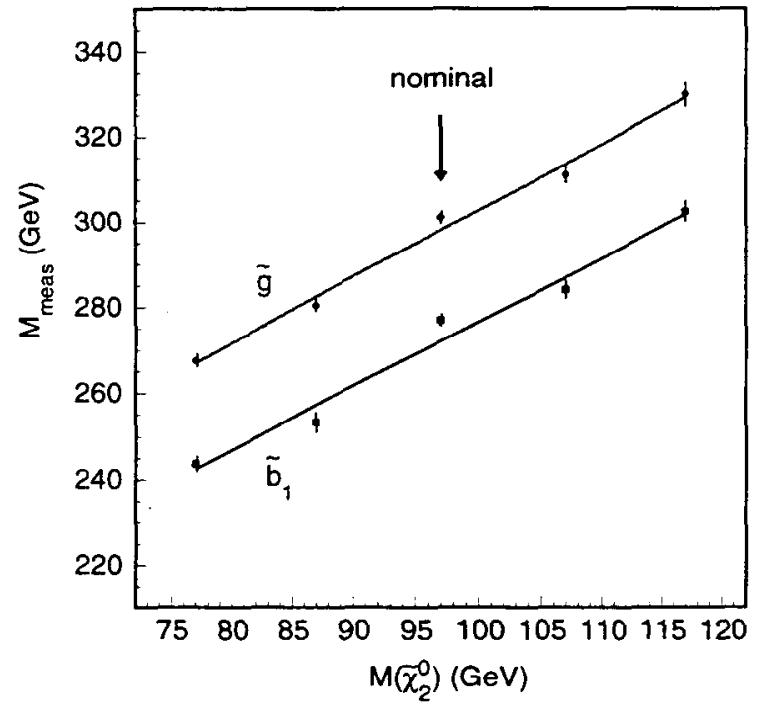

Figure 20-117 The dependence of the masses of $\tilde{b}_{1}$ and $\tilde{g}$ from the assumed value of the $\tilde{\chi}_{2}^{0}$ mass for Point $3\left(\lambda_{123} \neq 0\right)$. 
The distributions of the invariant masses for $\tilde{b}_{1}$ and gluino are very similar to the ones shown for $\lambda_{122} \neq 0$, once the $\tilde{\chi}_{2}^{0}$ mass is given. By repeating the analysis for different values of the $\tilde{\chi}_{2}^{0}$ mass a linear dependence, shown in Figure 20-117 was found between the $\tilde{b}_{1}$ and $\tilde{g}$ masses and the $\tilde{\chi}_{2}^{0}$ mass, which can be used as input to the fit of the fundamental parameters of the model.

\subsubsection{Constraints on SUGRA parameters}

For the $\bar{\chi}_{1}^{0}$ decays involving only leptons of the first two generations, the invariant masses of the many lepton combinations available, and the fact that the neutrino is massless make it possible to perform a direct reconstruction of some supersymmetric decay chains with good precision. In this case, therefore, the SUGRA parameters are constrained with higher precision than in the $R$-parity conserving case. For $L$-violating couplings involving $\tau$ leptons, no advantage can be gained from direct $\tilde{\chi}_{1}^{0}$ reconstruction, and the high combinatorial background of leptons from the $\bar{\chi}_{1}^{0}$ decay worsens in a significant way the precision of the measurements of edges in the invariant mass distribution of lepton pairs.

\subsubsection{Lepton number violation: $\chi_{1}^{0} \rightarrow q \bar{q} l, q \bar{q} v$}

When one of the $\lambda^{\prime}{ }_{i j k}$ is different from zero, each of the $\tilde{\chi}_{1}^{0}$ decays into two hadronic jets and a lepton, which can be either a charged lepton or a neutrino. The SUSY events will therefore exhibit a higher jet multiplicity than in the $R$-parity conserving case, with the presence of at least two additional leptons in each event. Two distinct final state phenomenologies appear in this case, depending on whether the lepton from the $\tilde{\chi}_{1}^{0}$ decay is a charged lepton or a neutrino. In the first case, one probably has the 'easiest' among all the possible $R$-parity violating signatures, as the two additional leptons per event allow easy separation from Standard Model backgrounds, and the two leptons can be taken as 'seeds' for the full reconstruction of the $\bar{\chi}_{1}^{0}$. In case the lepton is a neutrino, there is some $E_{T}^{\text {miss }}$ in the event, as shown in Figure 20-118, but hardly enough to guarantee the extraction of the signal from the background, and full reconstruction of $\tilde{\chi}_{1}^{0}$ is not possible.

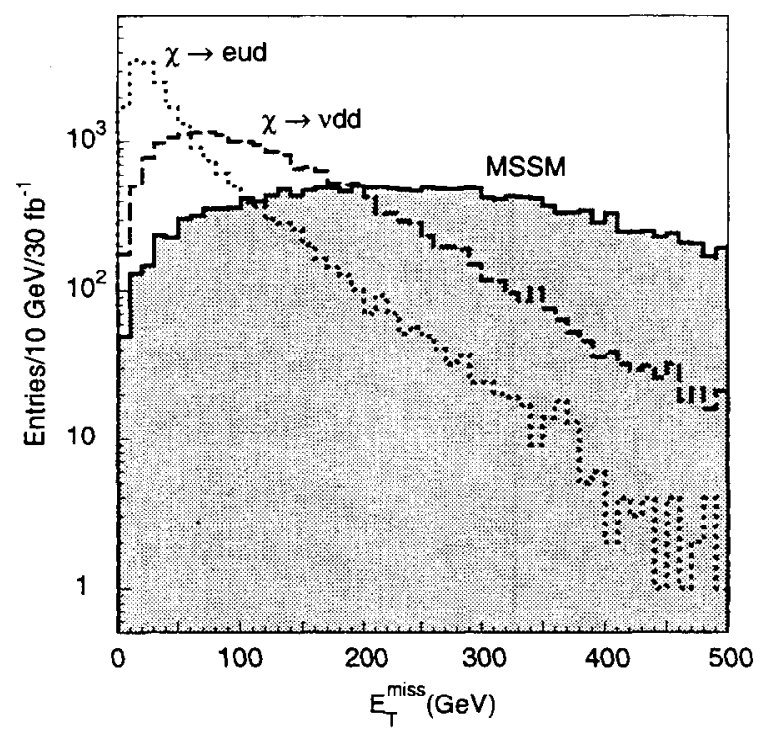

Figure 20-118 Comparison of the $E_{\mathrm{T}}^{\text {miss }}$ spectra for the $R$-parity conserving case, and for the decays into two jets and respectively a charged lepton or a neutrino.

For a given $\lambda_{i j k}^{\prime}$, both the decays $\tilde{\chi}_{1}^{0} \rightarrow q \bar{q} l$ and $\tilde{\chi}_{1}^{0} \rightarrow q \bar{q} v$ exist, with a relative branching fraction which is determined by the neutralino mixing matrix and the SUSY mass spectrum in the considered point of parameter space. The branching fractions for the five SUGRA points were calculated for the case $\lambda^{\prime}{ }_{112} \neq 0$ [20-45]. The branching fraction into charged leptons varied between $75 \%$ (Point 3 ) and $35 \%$ (Point 5 ), thus giving for all points a significant number of events with one or two charged leptons from $\tilde{\chi}_{1}^{0}$ decays in the final state. For detailed studies [20-46] 


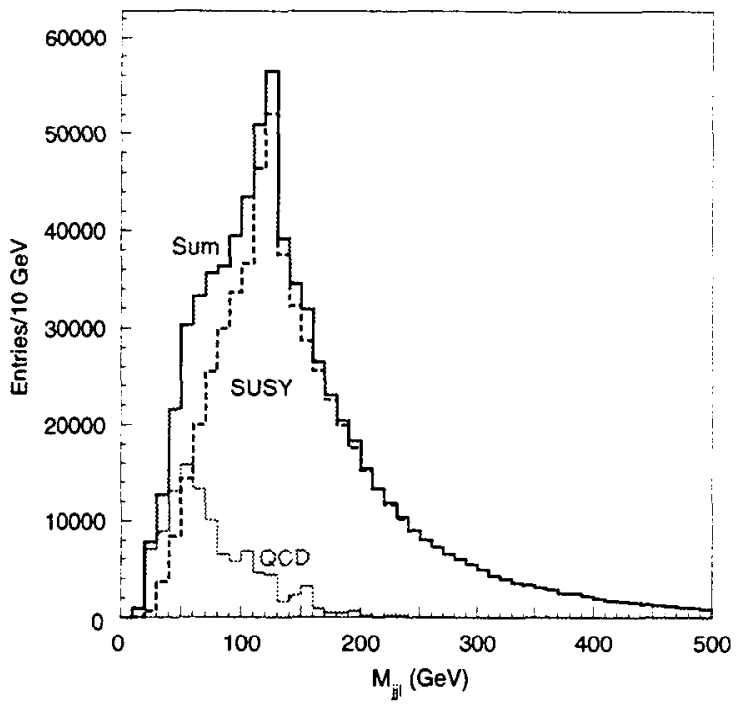

Figure 20-119 Invariant mass spectrum of jil combination for SUSY (dashed), QCD (shaded), and both (full line).
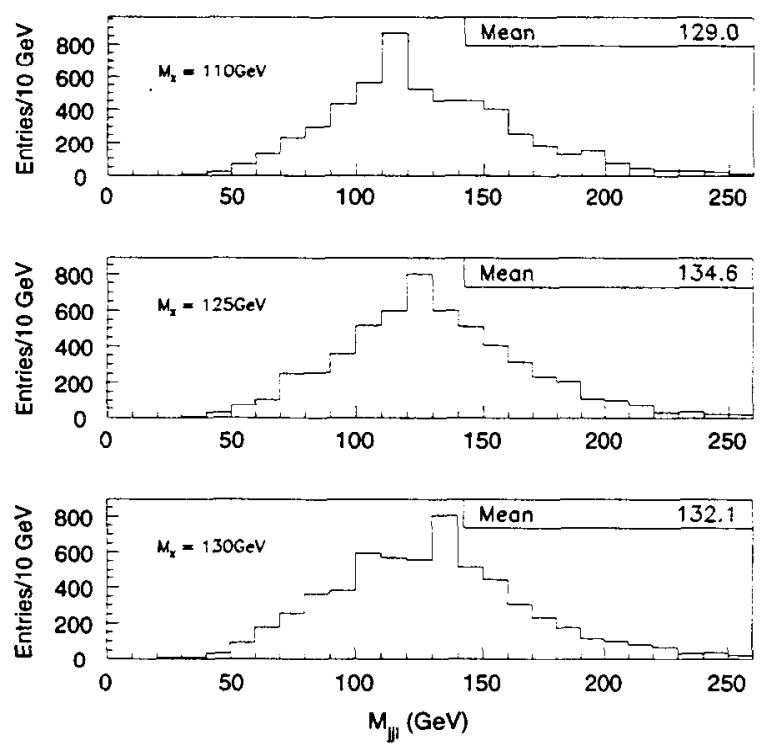

Figure 20-120 Invariant mass spectrum of $j$ li combinations for three values of the $\tilde{\chi}_{1}^{0}$ mass: $110 \mathrm{GeV}$ (top), $125 \mathrm{GeV}$ (middle), $130 \mathrm{GeV}$ (bottom).

only decays to the first generation were considered, and the two cases, respectively with the decay in electron or neutrino, were considered separately, assuming in each case a $100 \%$ branching fraction.

\subsubsection{Inclusive analysis}

The SUSY signal can easily be extracted from the SM background by exploiting the high charged lepton multiplicity in the final state, in the case of $\tilde{\chi}_{1}^{0} \rightarrow q \bar{q} l$ decays. This is true for all the five SUGRA points considered. For the decay $\tilde{\chi}_{1}^{0} \rightarrow e u \bar{d}$, requiring a jet multiplicity greater than seven, with a hard leading jet, plus a single lepton with $p_{\mathrm{T}}>15 \mathrm{GeV}$ is enough to obtain signal-to-background ratios of better than ten. Similarly to the $B$-violating case, the variable $m_{\mathrm{T}, \text { cent }}$ can be used to estimate the mass scale of the SUSY particles.

The decay $\tilde{\chi}_{1}^{0} \rightarrow v q \bar{q}$ could be dominant in some corners of the parameter space. To take this into account, the extraction of the SUSY signal was explicitly studied for this decay. In general, the limited amount of $E_{\mathrm{T}}^{\text {miss }}$ in the events does not allow the separation of the signal from the Standard Model background in a convincing way just by requiring high jet multiplicity and $E_{\mathrm{T}}^{\mathrm{miss}}$. As already seen for $B$-violating couplings, a signal to background ratio adequate for discovery can be obtained requiring the presence of at least one lepton in the final state. These leptons are abundantly produced in the cascade decays of sparticles over a significant fraction of the SUGRA parameter space.

\subsubsection{Precision measurements}

The strategy for precision measurement when the decay $\tilde{\chi}_{1}^{0} \rightarrow e u \bar{d}$ is dominant is radically different than in the $R$-parity conserving case. The starting point for the reconstruction of an exclusive decay chain is at the very bottom of the chain, where the presence of a charged lepton makes the reconstruction of at least one of the LSP reasonably easy. 


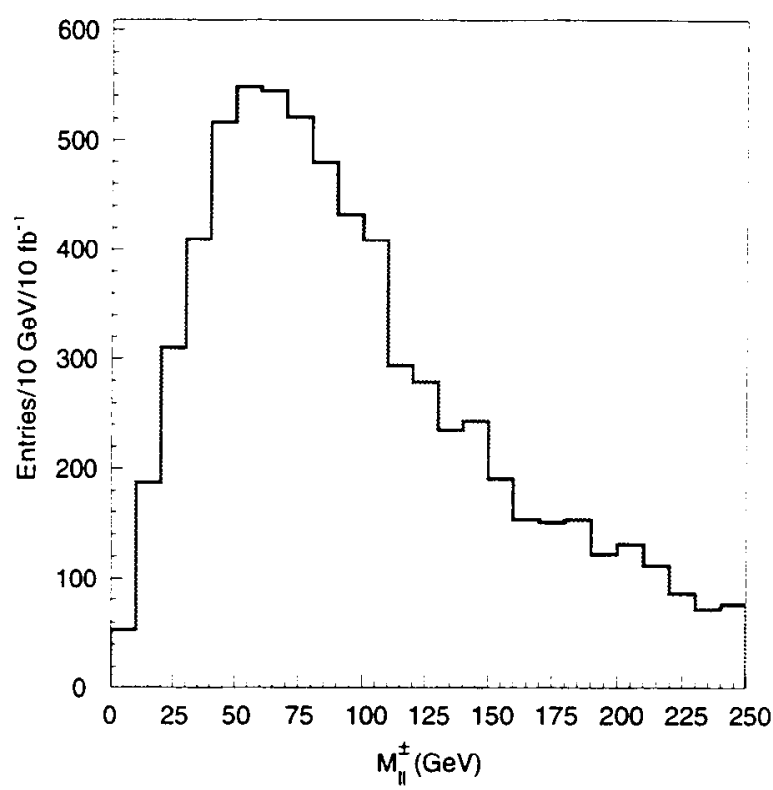

Figure 20-121 OS-SF lepton pairs invariant mass distribution (all leptons).

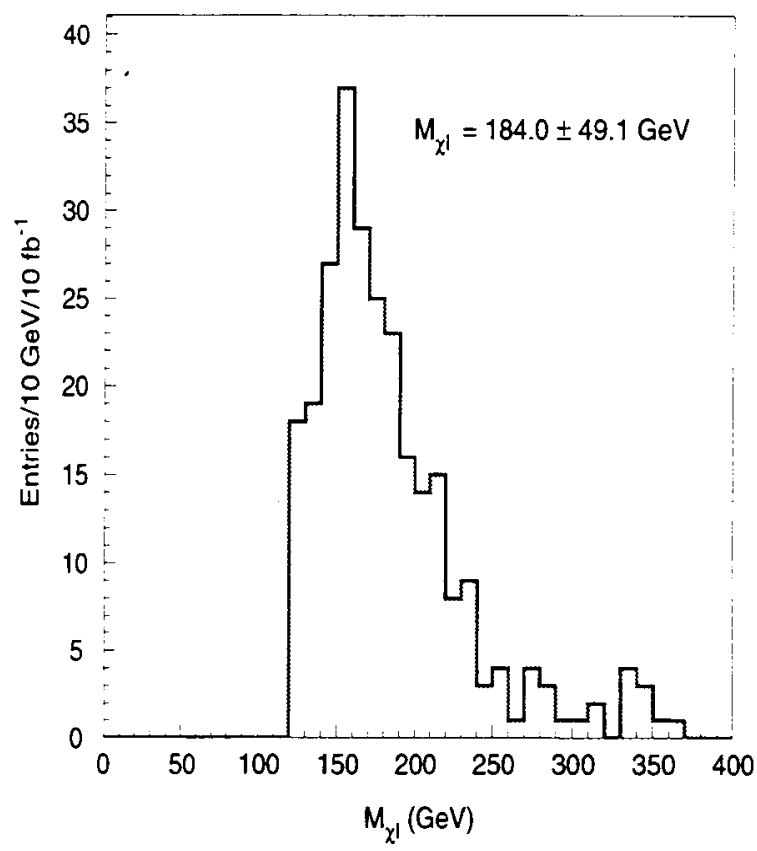

Figure 20-123 Minimum invariant mass distribution of the soft $\tilde{\chi}_{1}^{0}$ candidate combined with a lepton not used for $\tilde{\chi}_{1}^{0}$ reconstruction.

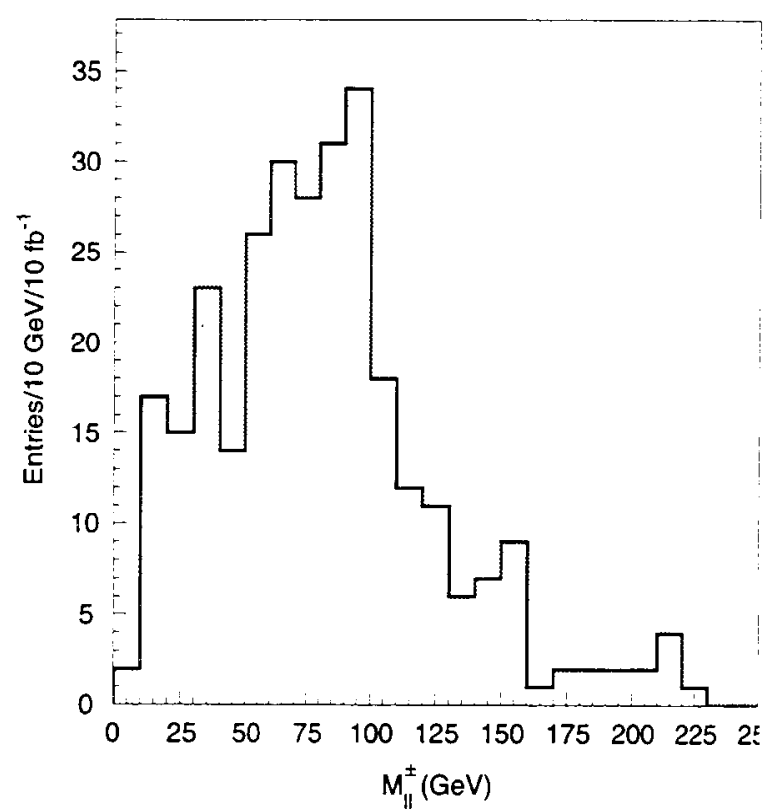

Figure 20-122 OS-SF lepton pairs invariant mass distribution (leptons not used for $\tilde{\chi}_{1}^{0}$ reconstruction).

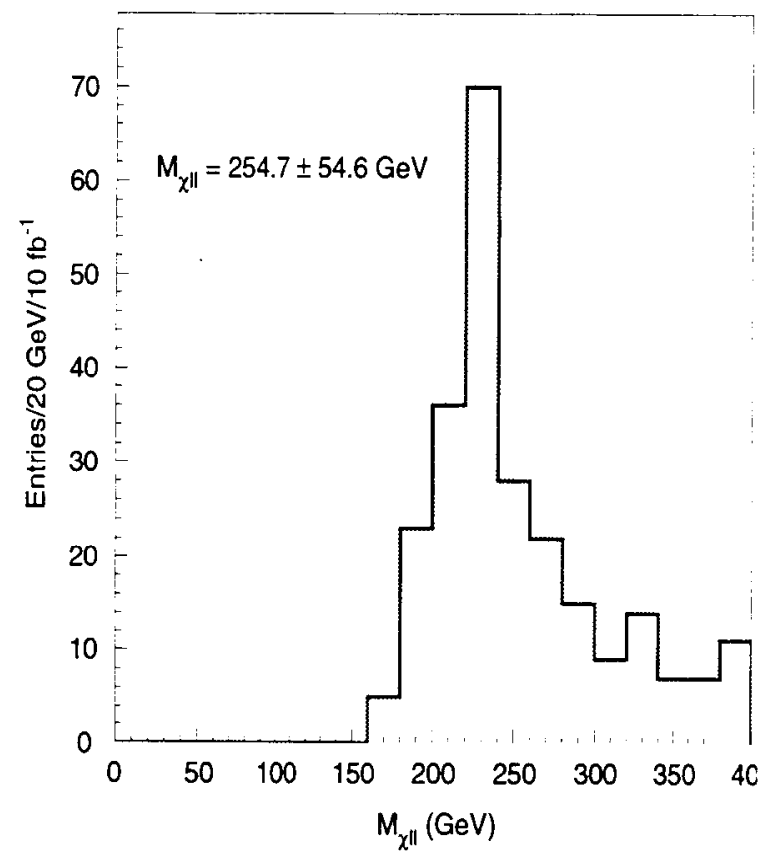

Figure 20-124 Invariant mass distribution of the soft $\tilde{\chi}_{1}^{0}$ candidate with two additional leptons. The peaks corresponds to the $\tilde{\chi}_{2}^{0}$ mass.

For SUGRA Point 5, the average $p_{\mathrm{T}}$ of the harder LSP is $320 \mathrm{GeV}$, and the decay particles have average $p_{\mathrm{T}}$ of 160,55 , and $105 \mathrm{GeV}$ for the two jets, and the charged lepton respectively, allowing to reach a good efficiency in the reconstruction of the $\bar{\chi}_{1}^{0}$.

The $\tilde{\chi}_{1}^{0}$ was reconstructed by first selecting the SUSY events with a set of loose cuts: 
- at least six jets with $p_{\mathrm{T}}>20 \mathrm{GeV}$, of which one with $p_{\mathrm{T}}>250 \mathrm{GeV}$;

- at least one lepton with $p_{\mathrm{T}}>20 \mathrm{GeV}$;

- transverse sphericity $>0.2$, and thrust $<0.9$.

Then the correct $j j l$ combination was chosen, out of the combinatorial background from jets directly produced in squark and gluino decays. For the leading $\tilde{\chi}_{1}^{0}$, the $\Delta R$ distance between the two jets was required to be smaller than 1 , and the distance between the leading (second) jet and the lepton was required to be smaller than 1 (1.5). All angular cuts were set at $\Delta R<2$ for the reconstruction of the second $\tilde{\chi}_{1}^{0}$. The combinatorial background was further reduced by requiring that the leading jet has $p_{\mathrm{T}}>50 \mathrm{GeV}$ and the second jet has $p_{\mathrm{T}}<100 \mathrm{GeV}$ for the first $\tilde{\chi}_{1}^{0}$, and both jets have $p_{\mathrm{T}}$ smaller than $100 \mathrm{GeV}$ for the second $\tilde{\chi}_{1}^{0}$. With these prescriptions 1.4 combination on average were found per event.

The invariant $j j l$ mass, shown in Figure 20-119, exhibits a clear peak at the $\tilde{\chi}_{1}^{0}$ mass. The observed peak is not generated by the kinematic cuts, since one can see that the QCD background does not exhibit a peak in the same position. Moreover the reconstruction was performed for different values of the $\tilde{\chi}_{1}^{0}$ mass. The displacement of the peak position, shown in Figure 20-120, allows the $\tilde{\chi}_{1}^{0}$ mass to be determined with a precision of $\pm 3 \mathrm{GeV}$. The same analysis was performed for SUGRA points 3 and 4, using the same angular and kinematic cuts. A peak, albeit degraded, is seen for Point 4 , whereas at Point 3 the mass of the $\tilde{\chi}_{1}^{0}$ is small ( $44 \mathrm{GeV}$ ) and the reconstruction from the very soft jets and leptons is problematic.

Once the $\tilde{\chi}_{1}^{0}$ has been reconstructed, it is possible to go up the decay chain and reconstruct other sparticle masses. As usual, for SUGRA Point 5 , the best decay chain for full exclusive reconstruction is $\tilde{q}_{L} \tilde{q}_{R}$ production, followed by the decays $\tilde{q}_{L} \rightarrow \tilde{\chi}_{2}^{0} q, \tilde{\chi}_{2}^{0} \rightarrow \tilde{l}_{R}^{t} l^{\mp} \rightarrow \tilde{\chi}_{1}^{0} l^{+} l^{-}$, and $q_{R} \rightarrow \tilde{\chi}_{1}^{0} q$. This decay offers a large branching fraction, a low jet combinatorial background, and the characteristic edge in the two-lepton invariant mass from the $\bar{\chi}_{2}^{0}$ decay to sleptons. In the case of the $\bar{\chi}_{1}^{0} \rightarrow e u \bar{d}$ decay, the edge is spoiled by the additional lepton combinatorics, as shown in Figure 20-121, where the two-lepton invariant mass is plotted for signal events. The situation can be improved by performing the $\bar{\chi}_{1}^{0}$ reconstruction, and considering only the events with two $j j l$ combinations with an invariant mass within $10 \mathrm{GeV}$ of the $\tilde{\chi}_{1}^{0}$ mass. The invariant mass of the opposite-sign same-flavour lepton pairs, excluding the leptons used for the $\tilde{\chi}_{1}^{0}$ reconstruction, is shown in Figure 20-122. The edge

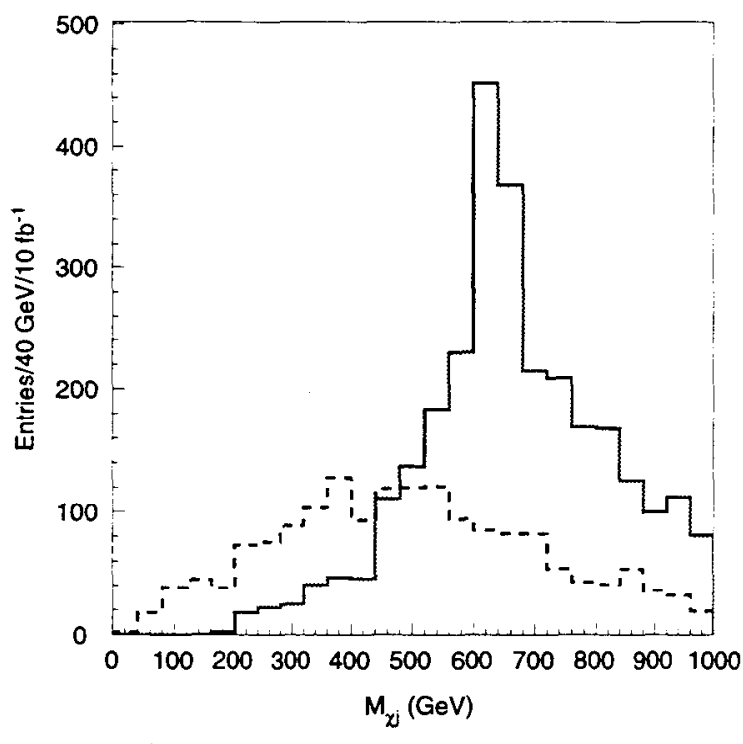

Figure 20-125 Invariant mass of the harder $\tilde{\chi}_{1}^{0}$ with the leading jet in the event. A broad peak can be seen around the nominal $\tilde{q}_{R}$ mass. The dashed distribution is the invariant mass of the softer $\tilde{\chi}_{1}^{0}$ with the second hardest jet in the event. at $100 \mathrm{GeV}$ is present, albeit much less clean than in the $R$-parity conserving case.

The events with two reconstructed $\tilde{\chi}_{1}^{0}$ can be used to reconstruct explicitly the $\tilde{l}_{R}$ mass by calculating the invariant mass of the $\tilde{\chi}_{1}^{0}$ with one of the two additional leptons. The problem is in this case the choice of the $\tilde{\chi}_{1}^{0}$ and of the lepton from the $\tilde{l}_{R}$ decay. The softer $\tilde{\chi}_{1}^{0}$ was used for the reconstruction, as it can be assumed that the harder one comes from the $\tilde{q}_{R}$ decay. The invariant 
mass of the softer $j j l$ combination was rescaled to the nominal $\tilde{\chi}_{1}^{0}$ mass, and the invariant mass with the remaining lepton was then calculated. The distribution of the lowest of the reconstructed invariant masses is shown in Figure 20-123. A peak can be seen corresponding to the slepton invariant mass of $157 \mathrm{GeV}$. By varying the slepton mass, a precision in the slepton mass measurement of $\pm 15 \mathrm{GeV}$ can be estimated. By further combining the reconstructed slepton with the remaining lepton, the $\tilde{\chi}_{2}^{0}$ can also be reconstructed. The distribution in Figure 20-124 was obtained by requiring the slepton candidate to have an invariant mass within $10 \mathrm{GeV}$ of the slepton mass. The statistics, for an integrated luminosity of $10 \mathrm{fb}^{-1}$ is limited, but a clear peak is observed at the nominal $\bar{\chi}_{2}^{0}$ mass of $228 \mathrm{GeV}$.

The mass of the $\tilde{q}_{R}$ can be measured through the decay $q_{R} \rightarrow \tilde{\chi}_{1}^{0} q$, by calculating the invariant mass of one of the reconstructed $\tilde{\chi}_{1}^{0}$ with a hard jet. Events were selected by requiring two reconstructed $\tilde{\chi}_{1}^{0}$ with a mass within $10 \mathrm{GeV}$ of the measured $\tilde{\chi}_{1}^{0}$ mass, and two hard central jets, within $|\eta|<2$, and with a $p_{T}$ respectively in excess of 300 and $100 \mathrm{GeV}$. The requirement of two hard jets is meant to preferentially select $\tilde{q}_{L} \tilde{q}_{R}$ production. Under the assumption that the SUSY sample is dominated by this process, the decay $q_{R} \rightarrow \tilde{\chi}_{1}^{0} q$ car be expected to yield the harder $\tilde{\chi}_{1}^{0}$ and the leading jet. The mass combination of the two is presented in Figure 20-125, which does indeed show a convincing peak corresponding to the position of the $\tilde{q}_{R}$ mass. In the same plot the dotted line shows the invariant mass of the softer jet combined with the softer $\tilde{\chi}_{1}^{0}$ which, on the contrary, as expected does not show any clear peak structure.

As in $R$-parity conserving Point 5 , the measurement of the lightest Higgs mass through the reconstruction of the $h \rightarrow b \bar{b}$ mass peak is necessary in order to constrain $\tan \beta$. The measurement is in this case more difficult due to the increased jet combinatorics from $\tilde{\chi}_{1}^{0}$ decay products when light jets are misidentified as $b$-jets. A narrow peak, albeit over a background much higher than in the $R$-conserving case, is obtained by selecting events with two reconstructed $\tilde{\chi}_{1}^{0}$, and excluding from the $b \bar{b}$ mass reconstruction jets which were used for the $\tilde{\chi}_{1}^{0}$. The situation would of course be worse, if the $\tilde{\chi}_{1}^{0}$ decays involved $c$ or $b$-quarks. Likewise, in the case of SUGRA Point 1 the much reduced statistics, together with the big efficiency loss produced by the requirement of two reconstructed $\tilde{\chi}_{1}^{0}$ could greatly reduce the significance of the $h \rightarrow b \bar{b}$ peak.

\subsubsection{Constraints on the SUGRA parameters}

The case with $\lambda^{\prime}{ }_{i j k} \neq 0$ is intermediate between the two previously considered cases. If the $\tilde{\chi}_{1}^{0}$ decay involves a lepton from the first two generations, SUSY can be very easily discovered. The $\tilde{\chi}_{1}^{0}$, and hence full SUSY decay chains, can be reconstructed. This reconstruction is based on two jets, and therefore can be performed only for a sufficiently heavy $\tilde{\chi}_{1}^{0}$, so as to have observable jets. The precision of the mass measurement is limited. Therefore, the advantage of this scenario with respect to $R$-parity conservation is the possibility of performing model independent mass reconstructions, whereas little improvement is expected in constraining the SUGRA parameters. On the other hand, as it happens for the $\lambda_{i j k} \neq 0$ case, the precision in the measurement of the lepton-lepton edge in Point 5 is spoiled by the high lepton multiplicity in the final state.

\subsection{Conclusion}

If SUSY exists at the weak scale, then its discovery at the LHC should be straightforward. The SUSY cross section is dominated by gluinos and squarks, which are strongly produced with cross sections comparable to the Standard Model backgrounds at the same $Q^{2}$. Gluinos and squarks then decay via a series of steps into the LSP (which may itself decay if $R$-parity is vio- 
lated). These decay chains lead to a variety of signatures involving multiple jets, leptons, photons, heavy flavours, $W$ and $Z$ bosons, and missing energy. The combination of a large production cross section and distinctive signatures makes it easy to separate SUSY from the Standard Model background. Therefore, the main challenge is not to discover SUSY but to separate the many SUSY processes that occur and to measure the masses and other properties of the SUSY particles. In most cases, the backgrounds from other SUSY events dominates the reducible Standard Model backgrounds due to the tails of the detector response. This fact justifies the use of particle level simulations for precision SUSY studies.

The number of free parameters for even the Minimal Supersymmetric Standard Model is very large, so it is impossible to investigate all cases. Since the background for SUSY is SUSY itself, one cannot just generate particular signals and compare them with known backgrounds. The approach followed here has been to investigate in detail the signatures for particular points in the parameter spaces of the minimal SUGRA, GMSB, and $R$-parity violating models. Methods such as looking for kinematic endpoints for mass distributions and using these to determine combinations of methods have proven generally useful. But each point has to some extent required inventing new analysis methods.

It seems clear that the points investigated here do not exhaust all possibilities even of the minimal SUSY models considered. Given the success in extracting precise measurements for these few points and the large number of SUSY events expected at the LHC, ATLAS is likely not just to discover SUSY if it exists but to make many precise measurements. The starting point in this study will be to look for characteristic deviations from the Standard Model. In SUGRA and some other models, there would be events with multiple jets and leptons plus large $E_{\mathrm{T}}^{\mathrm{miss}}$. In GMSB models, there would be events with prompt photons or quasi-stable sleptons. In $R$-parity violating models, there would be events with very high jet multiplicity and/or leptons. Any such signal would point to possible classes of models and would indicate the rough mass scale. The next step would be to use partial reconstruction methods like those described in this chapter to try to constrain as many combinations of masses as possible. A sharp dilepton edge like that in Figure 20-11 or a $h \rightarrow b \bar{b}$ peak is fairly unambiguous; so are some of the GMSB signatures. More complicated signatures, especially those involving complicated combinations of jets, would require much more work. These sorts of kinematical features represent only a small part of the total data. Given a SUSY signal, one would certainly generate large samples of events for many different models and compare many different distributions. This involves a huge effort, but one that would certainly be made given signals for new physics

The ultimate goal is to use all the measured quantities to determine the underlying SUSY model and to measure its parameters, just as the precision electroweak data has been fit in the Standard Model. The fits performed here have used minimal models with a small number of parameters. The fitting would clearly be more difficult for more complicated models. Even for simple models, it is not trivial to determine the masses and other parameters at a level of precision that matches many of the potential experimental measurements. Many corrections have to be included, and some can be quite important [20-47]. Fortunately - or rather unfortunately - we have many years to improve both the analysis techniques described here and the theoretical tools to interpret them.

\subsection{References}

20-1 J. Erler and P. Langacker, UPR-816-T, hep-ph/9809352 (1998). 
20-2 S. Weinberg, Phys. Rev. D13 (1976) 974, Phys. Rev. D19 (1979) 1277;

L. Susskind, Phys. Rev. D20 (1979) 2619.

20-3 Y. Gol'fand and E. Likhtam, JETP Lett. 13 (1971) 323;

P. Ramond, Phys. Rev. D3 (1971) 2415;

A. Neveu and J.H. Schwartz, Nucl. Phys. B31 (1971) 86;

J.L. Jervais and B. Sakita, Nucl. Phys. B34 (1971) 632;

D. Volkov and V. Akulov, Phys. Lett. B46 (1973) 109;

J. Wess and B. Zumino, Nucl. Phys. B78 (1974) 39.

20-4 General reviews of SUSY include H.P. Nilles, Phys. Rep. 111 (1984) 1;

H.E. Haber and G.L. Kane, Phys. Rep. 117 (1985) 75;

J. Wess and J. Bagger, Supersymmetry and Supergravity, ISBN 0691085560 (1992);

S.P. Martin, hep-ph/9709356 (1997).

20-5 G.W. Anderson and D.J. Castano, hep-ph/9409419, Phys. Lett. B347 (1995) 300.

20-6 M. Carena, et al., hep-ex/9712022 (1997) and references therein.

20-7 ALEPH Collaboration, Eur. Phys. J. C2 (1998) 417; Eur. Phys. J. C1 (1998) 1;

DELPHI Collaboration, Eur. Phys. J. C1 (1998) 1;

L3 Collaboration, Eur. Phys. J. C4 (1998) 207;

OPAL Collaboration, Eur. Phys. J. C2 (1998) 213.

20-8 ALEPH, DELPHI, L3, OPAL, and SLD Collaborations, CERN-PPE/97-154 (1997).

20-9 S.P. Martin, hep-ph/9602349, Phys. Rev. D54 (1996) 2340.

20-10 S. Dimopoulos and D. Sutter, Nucl. Phys. B452 (1995) 496.

20-11 L. Alvarez-Gaume, J. Polchinski, and M.B. Wise, Nucl. Phys. B221 (1983) 495;

L. Ibanez, Phys. Lett. 118B (1982) 73;

J. Ellis, D.V. Nanopolous, and K. Tamvakis, Phys. Lett. 121B (1983) 123;

K. Inoue et al., Prog. Theor. Phys. 68 (1982) 927;

A.H. Chamseddine, R. Arnowitt, and P. Nath, Phys. Rev. Lett. 49 (1982) 970.

20-12 For the form of the RGE's used here, see V. Barger, M.S. Berger, and P. Ohmann, hepph/9311269, Phys. Rev. D49 (1994) 4908.

20-13 M. Dine, W. Fischler and M. Srednicki, Nucl. Phys. B189 (1981) 575;

S. Dimopoulos and S. Raby, Nucl. Phys. B192 (1981) 353;

C. Nappi and B. Ovrut, Phys. Lett. 113B (1982) 175;

L. Alvarez-Gaume, M. Claudson, and M. Wise, Nucl. Phys. B207 (1982) 961;

M. Dine and A. Nelson, Phys. Rev. D48 (1993) 1227;

M. Dine, A. Nelson, and Y. Shirman, Phys. Rev. D51 (1995) 1362;

M. Dine, et al., Phys. Rev. D53 (1996) 2658.

20-14 H. Dreiner, hep-ph/9707435 (1997).

20-15 H. Baer, F.E. Paige, S.D. Protopopescu, and X. Tata, hep-ph/9305342 (1993); ibid., hep$\mathrm{ph} / 9804321$ (1998).

20-16 S. Mrenna, hep-ph/9609360, Comput. Phys. Commun. 101 (1997) 232.

20-17 E. Richter-Was, D. Froidevaux, and L. Poggioli, 'ATLFAST 2.0: a fast simulation package for ATLAS', ATLAS Internal Note ATL-PHYS-98-131 (1998).

20-18 H. Baer, C.-H. Chen, F.E. Paige, and X. Tata, Phys. Rev. D52 (1995) 2746; ibid., Phys. Rev. D53 (1996) 6241. 
The present LEP preliminary limits, presented at the ' 99 winter conferences, are ALEPH: 90.2 GeV (ALEPH 99-007 CONF-99-003, March 1999);

DELPHI: 95.2 GeV (DELPHI 99-8 CONF-208);

L3: $95.2 \mathrm{GeV}$ (L3 Notes 2382,2383, March 1999);

OPAL: $91.0 \mathrm{GeV}$ (OPAL Note PN382, March 1999).

20-20 For a recent review, see M.S. Turner, astro-ph/9901168; to be published in Proc. of Wein 98 (Santa Fe, 1998).

20-21 ATLAS Collaboration, Technical Proposal, CERN/LHCC/94-43 (1994).

20-22 I. Hinchliffe, F.E. Paige, M.D. Shapiro, J. Soderqvist, and W. Yao, Phys. Rev. D55 (1997) 5520.

20-23 E. Richter-Was, D. Froidevaux, and J. Soderqvist, 'Precision SUSY measurements with ATLAS for SUGRA Points 1 and 2', ATLAS Internal Note ATL-PHYS-97-108 (1997).

20-24 I. Hinchliffe, F.E. Paige, E. Nagy, M.D. Shapiro, J. Soderqvist, and W. Yao, 'Precision SUSY measurements at LHC: Point 3', ATLAS Internal Note ATL-PHYS-97-109 (1997).

20-25 F. Gianotti, 'Precision SUSY measurements with ATLAS: SUGRA Point 4', ATLAS Internal Note ATL-PHXS-97-110 (1997).

20-26 G. Polesello, L. Poggioli, E. Richter-Was, and J. Soderqvist, 'Precision SUSY measurements with ATLAS for SUGRA Point 5', ATLAS Internal Note ATL-PHYS-97-111 (1997).

20-27 D. Froidevaux, D. Cavalli, F. Gianotti, I. Hinchliffe, F. Paige, G. Polesello, L. Poggioli, E. Richter-Was, S. Resconi, M. Shapiro, J. Soderqvist, and A. Weidberg, 'Precision SUSY measurements with ATLAS: Extraction of model parameters and conclusions', ATLAS Internal Note ATL-PHYS-97-112.

20-28 S. George and T. Hansl-Kozaneka, eds., 'ATLAS Trigger Menus', ATLAS Internal Note ATL-DAQ-98-121 (1998).

20-29 H. Bachacou, I. Hinchliffe and F.E. Paige, 'Measurements of masses in SUGRA models at LHC', Atlas Internal Note ATL-COM-PHYS-99-017.

20-30 F. James, MINUIT Reference Manual, CERN Program Library Long Writeup D506.

20-31 N. Arkani-Hamed, J.L. Feng, L.J. Hall, and H.-C. Cheng, hep-ph/9704205, Nucl. Phys. B505 (1997) 3.

20-32 T. Tsukamoto, K. Fujii, H. Murayama, M. Yamaguchi, and Y. Okada, Phys. Rev. D51 (1995) 3153.

20-33 I. Hinchliffe and F.E. Paige, 'Measurements for SUGRA Models with Large $\tan \beta$ at LHC', ATL-COM-PHYS-99-018.

20-34 Y. Coadou, I. Hinchliffe, J. Lozano-Bahilo, L.C. Loveridge, and M.D. Shapiro, 'Identification of hadronic Tau decays in ATLAS', ATLAS Internal Note ATL-PHYS-98126.

20-35 X. Tata, private communication.

20-36 I. Hinchliffe and F.E. Paige, 'Measurements in Gauge Mediated SUSY Breaking Models at the LHC', hep-ph/9812233, ATL-PHYS-98-134.

20-37 L. Borissov, M. Leltchouk, F. Paige, J. Parsons, S. Rajogopalan, 'Study of non-pointing photon signatures of Gauge Mediated SUSY Breaking Models', Atlas Internal Note ATLCOM-PHYS-99-037 (1999). 
20-38 A. Nisati, S. Petrarca, and G. Salvini, hep-ph/9707376, Mod. Phys. Lett. A12 (1997) 2213; A. Nisati, 'Preliminary Timing Studies of the Barrel Muon Trigger System', ATLAS Internal Note ATL-DAQ-98-083 (1998).

20-39 G. Polesello and A. Rimoldi, 'Reconstruction of quasi-stable charged sleptons in the ATLAS muon spectrometer', Atlas Internal Note ATLAS-COM-MUON-99-008 (1999).

20-40 P. Richardson, private communication;

G. Corcella et al., Cavendish HEP-99/03 (1999).

20-41 J. Soderqvist, 'Consequences of Baryonic R-parity Violation for Measurements of SUSY Particles using the ATLAS Detector', ATLAS Internal Note ATL-PHYS-98-122.

20-42 L. Drage, M.A. Parker, 'Measurement of the LSP mass in supersymmetric models with $R-$ parity violation', Atlas Internal Note ATL-COM-PHYS-99-029.

20-43 A. Mirea and E. Nagy, 'Study of the determination of the SUGRA parameters using the ATLAS detector in the case of $L$-violating $R$-parity breaking', ATLAS Internal Note ATLCOM-PHYS-99-007.

20-44 A. Mirea, 'The program RPV_ISAJET, an extension of ISAJET for the case of R-parity violation', ATLAS Internal Note ATL-COM-PHYS-99-08.

20-45 E-J. Buis, 'Neutralino decay in SUGRA Point 4', ATLAS Internal Note ATL-COM-PHYS99-20.

20-46 A. Connors, PhD Thesis, University of Birmingham (UK), 1998.

20-47 See for example D.M. Pierce, J.A. Bagger, K. Matchev, and R. Zhang, hep-ph/9606211, Nucl. Phys. B491 (1997) 3. 